\title{
Impact of gut-derived metabollites on substrate metabolism and metabolic health
}

Citation for published version (APA):

Gonzalez Hernandez, M. A. (2020). Impact of gut-derived metabollites on substrate metabolism and metabolic health. [Doctoral Thesis, Maastricht University]. Maastricht University. https://doi.org/10.26481/dis.20201027mgh

Document status and date:

Published: 01/01/2020

DOI:

10.26481/dis.20201027mgh

Document Version:

Publisher's PDF, also known as Version of record

\section{Please check the document version of this publication:}

- A submitted manuscript is the version of the article upon submission and before peer-review. There can be important differences between the submitted version and the official published version of record.

People interested in the research are advised to contact the author for the final version of the publication, or visit the DOI to the publisher's website.

- The final author version and the galley proof are versions of the publication after peer review.

- The final published version features the final layout of the paper including the volume, issue and page numbers.

Link to publication

\footnotetext{
General rights rights.

- You may freely distribute the URL identifying the publication in the public portal. please follow below link for the End User Agreement:

www.umlib.nl/taverne-license

Take down policy

If you believe that this document breaches copyright please contact us at:

repository@maastrichtuniversity.nl

providing details and we will investigate your claim.
}

Copyright and moral rights for the publications made accessible in the public portal are retained by the authors and/or other copyright owners and it is a condition of accessing publications that users recognise and abide by the legal requirements associated with these

- Users may download and print one copy of any publication from the public portal for the purpose of private study or research.

- You may not further distribute the material or use it for any profit-making activity or commercial gain

If the publication is distributed under the terms of Article $25 \mathrm{fa}$ of the Dutch Copyright Act, indicated by the "Taverne" license above, 
- IMPACT OF GUT-DERIVED
METABOLITES ON

SUBSTRATE METABOLISM AND

METABOLIC HEALTH

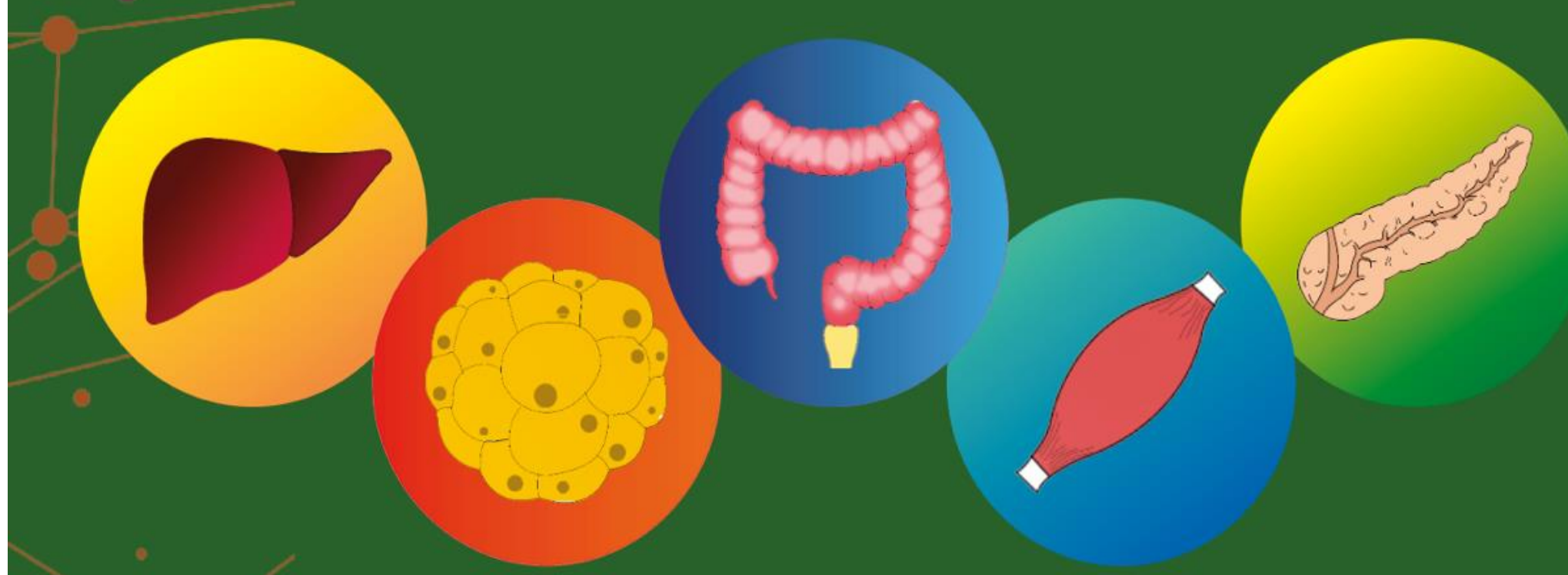


IMPACT OF GUT-DERIVED METABOLITES ON SUBSTRATE METABOLISM AND METABOLIC HEALTH 
This thesis was possible through a Scholarship granted to Manuel Gonzalez by Consejo Nacional de Ciencia y Tecnología (CONACYT), a public agency of Mexico's federal government.

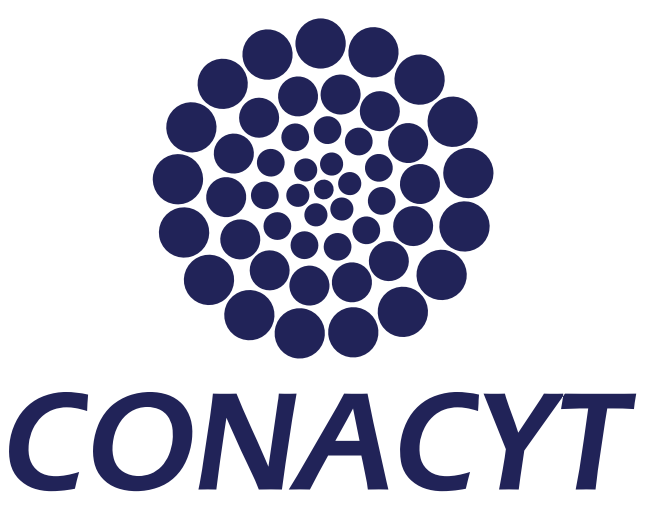

Consejo Nacional de Ciencia y Tecnología

Financial support by the Netherlands Association for the Study of Obesity (NASO) for the publication of this thesis is gratefully acknowledged.

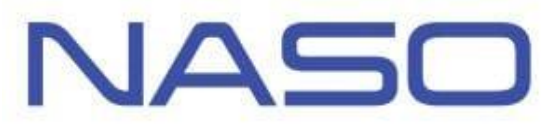

These studies presented in this thesis were performed within NUTRIM School of Nutrition and Translational Research in Metabolism, Maastricht University

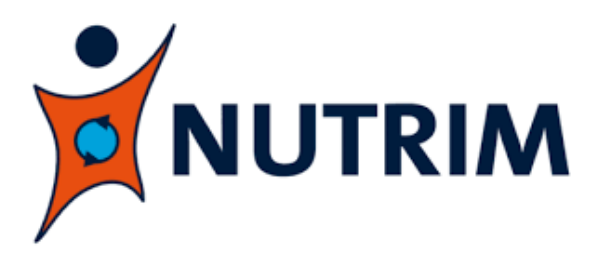

Cover design: Eduardo Lugo Gonzalez

Layout: Manuel Alejandro Gonzalez Hernandez

Printed by: Gildeprint

ISBN: 9789464190694

Manuel Alejandro Gonzalez Hernandez, Maastricht 2020 


\section{IMPACT OF GUT-DERIVED METABOLITES ON SUBSTRATE METABOLISM AND METABOLIC HEALTH}

\section{DISSERTATION}

to obtain the degree of Doctor at the Maastricht University, on the authority of the Rector Magnificus, Prof. dr. Rianne M. Letschert, in accordance with the decision of the Board of Deans, to be defended in Public on Tuesday $27^{\text {th }}$ October 2020, at 13:00 hours

by

\section{Manuel A. González Hernández}

born on May 12 1989 in León Guanajuato, México 


\section{Promotor}

Prof. dr. E.E. Blaak

\section{Co-promotors}

Dr. Emanuel E. Canfora

Dr. Johan W.E. Jocken

\section{Assessment committee}

Professor Dr M.A. van Baak, Emeritus Professor of the department of Human Biology - Maastricht University

Professor Dr K. Venema, Professor of Gut Microbiology. Department of Human Biology/ Venlo Campus - Maastricht University

Dr. G.D.A. Hermes, Senior Researcher of the department of Agrotechnology and Food Sciences - Laboratory of Microbiology - Wageningen University

Dr. F.J. Troost, Associate Professor of Food Innovation and Health, Centre for Healthy Eating and Food Innovation, campus Venlo - Maastricht University

Professor Dr. P.C.N. Rensen, Professor of Endocrinology at the Leiden University Medical Center 


\section{Table of contents}

$\begin{array}{lll}\text { Chapter 1. General introductios } & \text { G }\end{array}$

Chapter 2. The short chain fatty acid acetate in body weight control and insulin sensitivity

Chapter 3. Relation between circulating acetate and human insulin resistance before and after weight loss in the DiOGenes study

Chapter 4. Circulating but not fecal short-chain fatty acids are related to insulin sensitivity, lipolysis and GLP-1 concentrations in humans

Chapter 5. Fecal microbial metabolites of proteolytic and saccharolytic fermentation in relation to degree of insulin resistance in overweight and obese individuals

Chapter 6. Acetate does not affect palmitate oxidation and AMPK phosphorylation in human primary muscle cells

Chapter 7. Short-chain fatty acids differentially affect intracellular lipolysis in a human white adipocyte model

Chapter 8. General discussion

Appendix.

Summary

Resumen

Valorization

Acknowledgements

Curriculum 

CHAPTER 1

General introduction 


\section{The global obesity epidemic}

In the last decades, the prevalence of overweight adults has increased approximately 2 billion, of which 650 million are obese, according to the wor health organization (WHO) 2016 report ${ }^{1}$. Of note, prediction models estimate that the prevalence of obesity will further rise in the following decades ${ }^{2}$. Excess body weight is mainly classified using the body mass index (BMI) which is defined as body weight $(\mathrm{kg})$ divided by the square of height $\left(\mathrm{m}^{2}\right)$. Overweight $\left(>25 \mathrm{~kg} / \mathrm{m}^{2}\right)$ and obesity $\left(>30 \mathrm{~kg} / \mathrm{m}^{2}\right)$ phenotypes are characterized by increased adiposity that may link to adipose tissue dysfunction and the development of insulin resistance, Type 2 diabetes Mellitus (T2DM) and cardiovascular disease (CVD) ${ }^{1}$. However, the BMI as a unique measurement of obesity does not reflect the whole complexity of the disease. The major limitation of BMI is that it cannot distinguish fat from lean body mass nor metabolically benign from metabolically harmful fat at the individual level. Thus, BMI should be interpreted with caution 3. Importantly, obesity is a risk factor for the development of sleep apnea, stroke, gallbladder disease, hyperuricemia, hypertension, dyslipidemia, gout, osteoarthritis and certain types of cancer among others ${ }^{4,5}$.

Increased physical inactivity together with a relatively high consumption of energy-dense foods are major drivers of the energy imbalance that leads to the obesity epidemic. In addition, other factors may play a role in obesity and comorbidities, including stress, sleep, genetic profile, epigenetic modifications, ${ }^{6}$ social determinants ${ }^{7}$ as well as microbiota composition and functionality. Nowadays the territories with highest prevalence (between 28-36\%) of obesity include the United States of America, Mexico, some parts of Europe and the Middle East ${ }^{8}$. Based on this, there is no question that obesity and its comorbidities pose a risk with potential catastrophic consequences for global health as well as a menace for various nations' economies ${ }^{9}$. Currently, there are three pillars in the therapeutic approaches to combat obesity, including lifestyle modifications (nutritional advice, physical activity), bariatric surgery and pharmacotherapy ${ }^{10}$. Unfortunately, weight is partially or fully regained in most of the individuals $(\sim 80 \%)$ who undergo a weight loss intervention ${ }^{11,12}$. In response, vast literature suggests that successful interventions may require continuous monitoring support and behavioral therapy to the obese individuals from the health care providers ${ }^{13-16}$. It has become increasingly clear that intervention success may vary between individuals ${ }^{17-19}$ and that addressing the multifactorial etiology of overweight/obesity from different angles (multidisciplinary approach) is needed ${ }^{20}$. Therefore, more targeted or personalized strategies considering individual preferences, genetic, microbial and metabolic phenotypes may possibly increase the effectiveness of a weight loss intervention and increase the improvement in metabolic health. On this matter, the role of the gut microbial community and gut-derived metabolites in the etiology of obesity and cardiometabolic diseases as putative targets for 
nutritional interventions have received increasing attention in the past two decades ${ }^{21-23}$.

The gut microbiota consists of 10-100 trillion microbial cells ${ }^{24}$, and so far 500-1000 bacterial species have been discovered that ferment dietary components ${ }^{25}$. Mainly in the colon, indigestible carbohydrate (saccharolytic) fermentation by the microbial community produces short chain fatty acids (SCFA) as well as other metabolites (i.e. lactate, succinate) and gases (i.e. methane, carbon dioxide and hydrogen). Major SCFA are acetate, propionate and butyrate, which have been reported to have beneficial effects on metabolic health ${ }^{26,27}$. Saccharolytic fermentation takes place mainly in the proximal colon and decreases towards the distal colon as observed in sudden death victims ${ }^{28}$. In case of a diet that is low in complex fermentable carbohydrates, the distal portion of the colon specializes more in proteolytic fermentation (residual peptides and proteins). Although proteolytic fermentation may produce SCFA, its contribution to SCFA levels remains largely unknown ${ }^{25}$. In addition, proteolytic fermentation yields the production of a wide variety of metabolites including phenolic compounds, indoles, hydrogen sulfide as well as branched chain fatty acids (BCFA) such as isovalerate and isobutyrate ${ }^{25,29,30}$. Of note, many of these proteolytic compounds are of toxic nature and if produced in high quantities may have adverse effects on gut and metabolic health 25,31 . In turn, more insight into relevant gut microbial metabolites (SCFA/BCFA) is needed to elucidate their effects in host metabolism (saccharolytic/proteolytic balance) and understand their role in the whole-body and tissue specific metabolism in humans.

\section{Microbial community and gut functioning}

The gut microbial community is highly interconnected and actively produces various metabolites mainly as a result of saccharolytic or proteolytic fermentation along the colon 25-27, 29,30. The gut microbial community is shaped throughout life by various factors including birth delivery mode ${ }^{32}$, diet ${ }^{33-36}$, antibiotics 37 , environment 38,39 , temperature ${ }^{40}$ among other factors. A core microbial community consists of various microorganisms, but the most studied group are the gut bacteria, classified by the dominant phyla Bacteroidetes, Firmicutes, Proteobacteria, Verrucomicrobia, and Actinobacteria ${ }^{41}$. It is important to consider various aspects about the core microbial community, such as diversity, ecological robustness, taxonomic classifications (species, phylum level) as well as the functionality of the microbial networks ${ }^{42,43}$. A high microbial diversity has been linked to a healthy gut as well as to a better insulin sensitivity and metabolic health in various animal and human studies ${ }^{44-49}$. Although healthy gut functioning is not clearly defined, there are various factors that determine gut health and host metabolic health including the maintenance of gut permeability (this is the passage of metabolites from the gut lumen to the 
basolateral site which is importantly regulated by tight junction proteins), microbial SCFA production, antimicrobial molecules, gut-derived hormone secretion and maintenance of mucus layer thickness (gut barrier function) ${ }^{50-52}$. Under pathological conditions, a symphony of factors are linked to gut dysbiosis including an increased gut permeability (the so called "leaky gut") causing lowgrade inflammation (i.e. liver) ${ }^{51,53}$, a decrease in microbial diversity ${ }^{54-57}$, increments in pathogenic bacteria (i.e. proteobacteria) ${ }^{51,58}$ and translocation of pathogenic bacteria to portal vein and peripheral blood ${ }^{59}$. With respect to proteolytic fermentation products (i.e. BCFA), some of these compounds may have detrimental effects on colonic and metabolic health under conditions of a high-protein consumption ${ }^{25}$, ${ }^{6}$. In general, gut microbiota composition and functionality as well as gut permeability and gastro-intestinal transit are interlinked and have been connected with the metabolism of various organs including the liver ${ }^{25,59,61}$, skeletal muscle ${ }^{62,63}$, adipose tissue ${ }^{23}$, pancreas ${ }^{64,65}$ and brain ${ }^{25}$; that collectively can affect whole-body substrate and energy metabolism, inflammatory profile and insulin sensitivity ${ }^{66,67}$. Furthermore, there are compositional characteristics of the gut microbiota (at the genus/species level) that are associated with host metabolic health 68,69 . Of note, these alterations may improve microbial network functionality through increments in abundance of amongst others bifidobacteria (bifidogenic effect), butyrate-producing bacteria (i.e. Faecalibacterium, Ruminococcus) and Akkermansia muciniphila (acetate producer) ${ }^{70,71}$.

Under the premise to restore gut and metabolic health, interventions targeting the microbiome (i.e. probiotics or prebiotics) have been performed. In this regard, it is well recognized that prebiotic interventions (i.e. fructooligosaccharides (FOS), galacto-oligosaccharides (GOS), arabinoxylan oligosaccharides (AXOS), inulin) of various durations (1-12 weeks) can have a bifidogenic effect ${ }^{34,72-74}$, however, their effects on metabolic health have been inconsistent, especially data on SCFA production and gut-derived hormone secretion. Currently, considerable attention is given to postbiotics (i.e. SCFA) as they have shown a potential benefit in body weight control and improvements in insulin sensitivity ${ }^{75}$. However, little data are available on the role of SCFA in human substrate and energy metabolism, in particular their role in more metabolically compromised phenotypes which is still not fully understood. Furthermore, little is known about products of proteolytic fermentation, such as BCFA (isovalerate, isobutyrate) in humans. 


\section{Short chain fatty acid metabolism}

Colonic SCFA production is modulated by various microbial pathways that produce acetate (acetogenesis and carbon fixation pathways) ${ }^{33}$, propionate (acrylate pathway) ${ }^{25}$ and butyrate (butyrogenic pathways) ${ }^{76}$. These processes, the abundance of specific bacterial species (i.e. Akkermansia muciniphila, Bacteroides thetaiotaomicron, Faecalibacterium prausnitzii) ${ }^{23,31}$, bacterial crossfeeding mechanisms (mainly from acetate to butyrate, and to a lower extent from butyrate to propionate) ${ }^{77}$ in combination with diet (i.e. fiber characteristics) ${ }^{78}$ play a prominent role in SCFA production. In theory, fermentation of $10 \mathrm{~g}$ of indigestible carbohydrates can produce approximately $100 \mathrm{mmol}$ of SCFA in the colon ${ }^{79}$. Total production of SCFA has shown a molar ratio of roughly 3:1:1 as observed in fecal levels of acetate, propionate and butyrate, respectively ${ }^{36}$. Moreover, a study in sudden death victims, showed that SCFA concentrations decrease from the ascending, to the transverse, descending and sigmoid colon ${ }^{28}$, indicating higher production in the proximal colon $(123 \mathrm{mmol} / \mathrm{kg}, 117 \mathrm{mmol} / \mathrm{kg}$, $80 \mathrm{mmol} / \mathrm{kg}$ and $100 \mathrm{mmol} / \mathrm{kg}$, respectively). According to kinetic studies on SCFA metabolism, SCFA follow a preferential order in utilization by colonic epithelial cells, starting with butyrate $>$ propionate $>$ acetate ${ }^{80,81}$. In particular, butyrate plays an important role as an energy source for colonocytes and colonic mucosa ${ }^{82}$ while particularly acetate and to a significant lower extent propionate can reach the portal vein and systemic circulation in relatively high concentrations ${ }^{63}$. In addition, in vivo human studies using tracers have reported that colonic SCFA production is nearly matched by liver uptake for butyrate and propionate, whereas acetate can reach to a higher extent the systemic circulation 83, 84. In general, colonic SCFA absorption is concentration-dependent from the gut lumen to the periphery and circulating levels are impacted by colonic production and absorption rate ${ }^{85}$. Briefly, SCFA absorption is regulated by various mechanisms including hydrogen/sodium coupled monocarboxylate transporters (MCT), exchange with bicarbonate and non-ionic diffusion ${ }^{86-88}$. After colonic absorption, SCFA may reach the systemic circulation in different concentrations (acetate 19-450 $\mu \mathrm{mol} / \mathrm{L}$, propionate 1-13 $\mu \mathrm{mol} / \mathrm{L}$ and butyrate 1$12 \mu \mathrm{mol} / \mathrm{L}){ }^{36,89}$. Interestingly, a study in men conducted during abdominal surgery reported a higher SCFA release from distal colon compared to proximal colon, indicating that colonic absorption may differ between colonic segments ${ }^{90}$. Acetate was significantly released only from distal colon. Of note, the implications of these findings are that targeting the distal colon with slowly fermentable fibers may increase circulating SCFA and acetate concentrations, which have been previously shown to translate into more pronounced metabolic effects ${ }^{91,92}$. In the colon, SCFA may modulate gut and host metabolism through G-protein coupled receptors (GPR) 41/43 ${ }^{93-96}$, whilst SCFA may also affect metabolism through entry into the systemic circulation affecting thereby 
peripheral insulin sensitive tissues that express GPR (liver, skeletal muscle, adipose tissue and pancreatic beta cells) ${ }^{97-99}$.

In addition, a study conducted during abdominal surgery also reported a significant liver uptake of all SCFA ${ }^{90}$ which highlights the potential significance of SCFA for hepatic metabolic processes (i.e. lipogenesis, gluconeogenesis) ${ }^{100-102}$. With respect to measurements of SCFA concentrations, the majority of human (and murine) studies have measured SCFA levels in fecal material ${ }^{86,103-105}$ as a surrogate marker for SCFA colonic production. In this regard, it is important to consider that gut SCFA absorption is efficient ${ }^{106,107}$, and that fecal levels depend on various factors including microbial community ${ }^{108}$, intestinal transit time ${ }^{109}$, diet ${ }^{110,111}$, age ${ }^{112}$, lifestyle ${ }^{113}$ among others. Only a few human studies (mainly with an exploratory focus) have measured SCFA concentrations in the circulation $28,29,83,91,92,114$. Therefore, further studies should fully elucidate to what extent fecal and/or plasma SCFA reflect colonic SCFA production and to what extent they relate to metabolic health markers. In addition, mechanistic studies are needed using human derived tissue from biopsies and/or cell lines from relevant tissues (liver, adipose tissue, muscle) to identify tissue specific effects of SCFA in host metabolic health.

\section{Branched chain fatty acid metabolism}

The gut microbial BCFA isovalerate, isobutyrate and 2-methylbutyrate are mainly derived from proteolytic fermentation of branched chain amino acids (BCAA, leucine, isoleucine and valine), through the action of specific bacterial genera (i.e. Bacteroides, Clostridium, Fusobacterium) ${ }^{25,115}$. The abundance of BCFA producing bacteria decreases when distal colonic microbes are able to switch to saccharolytic fermentation as a result of an increased carbohydrate availability ${ }^{116}$. In humans following a Western diet, approximately $12 \mathrm{~g}$ of amino acids and peptides from both dietary and endogenous origin escape digestion in the small intestine and reach the colon daily ${ }^{117}$. This amount increases proportionally with an increased intake of plant-based protein or an overall increased protein intake from animal sources (such as cow's milk, beef and cheese) ${ }^{118-120 . ~ I n ~ p a r t i c u l a r, ~}$ plant-based proteins may be less digestible and more fermentable (as compared to animal-based proteins): for instance brown beans have reported to increase the plasma BCFA isobutyrate concentration in humans ${ }^{121}$. In humans, total colonic BCFA production is estimated around $18.87 \mathrm{mmol} / \mathrm{kg}$ of dry matter ${ }^{25}$. As previously explained, the distal colon microbiota specializes more in proteolytic fermentation to produce BCFA 25, 29, 60, 122. In support, an in vitro system using gut contents from distal and proximal human colons, reported that BCFA concentrations were higher in the distal than in the proximal colon (6.3 and 4.5 $\mathrm{mmol} / \mathrm{L}$, respectively) ${ }^{123}$. Moreover, a seminal study in sudden death victims showed that BCFA colonic concentrations vary along the different segments of 
the large intestine ${ }^{28}$. Isovalerate concentrations were $2.7,3.4,3.5$ and $3.7 \mathrm{mmol} / \mathrm{kg}$ and isobutyrate concentrations were $1.8,2.6,2.3$ and $1.9 \mathrm{mmol} / \mathrm{kg}$ in ascending, transverse, descending and sigmoid colon, respectively ${ }^{28}$.

In healthy individuals, fecal concentrations of isobutyrate and isovalerate have been reported $105 \pm 67$ and $178 \pm 126 \mu \mathrm{g} / \mathrm{g}$ of dry weight, respectively ${ }^{124}$. In the colon, isovalerate can activate the olfactory receptor 558 (Olfr558) as observed in a murine colonic organoid system ${ }^{125}$. Of note, OR51E1 the human ortholog of Olfr558 is expressed in human enteroendocrine L cells, thus suggesting a potential effect of BCFA isobutyrate to induce gut hormone secretion ${ }^{126}$. After colonic absorption, BCFA reach the circulation as observed in individuals following emergency surgery, which reported that BCFA concentrations in arterial and portal vein were $43.7,41.7 \mu \mathrm{mol} / \mathrm{L}$ and $21.1,15.5$ $\mu \mathrm{mol} / \mathrm{L}$ for isovalerate/2-methylbutyrate and isobutyrate, respectively, ${ }^{123}$. In the systemic circulation, isovalerate may regulate blood pressure via the Olfr558, which is highly expressed in blood vessels (aorta), heart and kidney. While in other tissues, such as large intestine, brain, lung and liver expression levels of Olfr558 are low, as observed in mice ${ }^{127}$. With respect to isobutyrate, it has been reported to act selectivity towards GPR41 in immune cells ${ }^{93}$, and given the broad expression profile of GPR41 ${ }^{36}$, it remains a challenge to pinpoint tissue specific functions. Interestingly, an in vitro study using rat and human adipocytes showed that both BCFA (isobutyrate and isovalerate) inhibited cytosolic (intracellular) lipolysis and lipogenesis using supraphysiological concentrations $(10 \mathrm{mmol} / \mathrm{L})^{128}$.

Interestingly, a cross-sectional study in humans showed that total fecal BCFA (isovalerate, isobutyrate and 2 methylbutyrate) concentration was higher in obese individuals (30-37 BMI) compared to individuals with a normal weight ${ }^{129}$. This was attributed to a higher proteolytic fermentation, as suggested by high BCFA together with a tendency for increased ammonium and phenolic concentrations in feces. In summary, little is known on the role of gut microbialderived BCFA in tissue specific and whole-body metabolism, substrate utilization and energy homeostasis in humans. In the last section, we discuss the available data on the relationship of BCFA with the control of body weight and insulin sensitivity. 


\section{Interorgan crosstalk in obesity and insulin resistance}

Excessive body weight linked to ectopic fat accumulation increases the risk of developing metabolic disturbances in glucose homeostasis and insulin sensitivity. Increased adiposity is characterized by enlarged (hypertrophic) adipocytes with large lipid droplets (in the form of triacylglycerols) in the white adipose tissue (WAT) ${ }^{130}$. Metabolic disturbances include increased triacylglycerol (TAG) and lipid metabolite (i.e. ceramides, diglycerides, long chain acyl-CoA and acylcarnitines) accumulation in non-adipose tissues (i.e. skeletal muscle, liver) ${ }^{131}$ and in the circulation ${ }^{132,}$, 133, an impaired lipid buffering capacity of adipose tissue and lipid spillover (TAG, free fatty acids) ${ }^{130}$.

As indicated, adipose tissue shows an impaired lipid uptake and reduced lipogenesis in obese, insulin resistant conditions when its storage capacity is exceeded ${ }^{134}$. The lipid spillover to the systemic and portal circulation leads to an increase in lipid supply to other tissues such as the liver, skeletal muscle and pancreas. Furthermore, fat oxidation rates in skeletal muscle, liver and adipose tissue fail to match the constant lipid supply, a phenomenon indicated as metabolic inflexibility ${ }^{135}$. According to the adipose tissue expandability hypothesis, its expandability is determined by both genetic and environmental factors ${ }^{136,137}$, and as the adipose tissue expands, immune cell infiltration (i.e. macrophages, $\mathrm{T}$ cells) increases and through the secretion of proinflammatory adipokines (e.g tumor necrosis factor alpha, $\mathrm{TNF} \alpha$ ) insulin signaling is altered 138, 139. Additionally, the liver and skeletal muscle may secrete TNF $\alpha$ locally ${ }^{139}$ as well as several other bioactive molecules (hepatokines and myokines, respectively) with distinct effects on insulin sensitivity. However, the exact mechanisms still need to be fully elucidated ${ }^{140}$. Chronic dyslipidemia may result in a metabolic dysfunction of the liver, known as non-alcoholic fatty liver disease (NAFLD), which may develop into a fibrotic, cirrhotic and insulin resistant liver 141, 142. Intracellular lipids, in particular bioactive lipid metabolite (i.e. diacylglycerol and ceramides) accumulation in liver and skeletal muscle may alter insulin signaling, glycogen storage and glucose handling ${ }^{143,}{ }^{144}$. Skeletal muscle is recognized as a relevant organ in glucose disposal, since it can enhance glucose clearance for almost $80 \%$ in the postprandial state ${ }^{145}$. In addition, an insulin resistant state may develop in a tissue specific manner (liver, skeletal muscle, adipose tissue) ${ }^{19}$. Alterations in pancreas become apparent when pancreatic beta cells fail to cope with increased insulin demands as a compensation for the progressing insulin resistance. First of all, enhanced insulin secretion compensates for the progressing insulin resistance in order to maintain glucose homeostasis despite pancreatic lipid accumulation and beta cell failure 146. 
Secondly, impaired insulin secretion from beta cells and increased wholebody insulin resistance result in altered glucose homeostasis and development of the prediabetic and diabetic state. In addition, the gastrointestinal tract is of major importance for the human substrate and energy metabolism, since it is in first contact with food and its main functions include to digest and absorb food, obtain energy, secrete gut hormones (peptide YY, PYY and glucagon like peptide, GLP-1) and ferment metabolites that escape digestion in the small intestine. Collectively, gut functioning and gut-derived products maintain whole-body homeostasis through their effects on energy and substrate metabolism.

\section{Role of SCFA in the interorgan crosstalk}

As explained above, insight has increased on gut-derived metabolites (SCFA) in particular in their role in the interorgan cross talk with the aforementioned tissues.

In the colon, SCFA may modulate various mechanisms that can affect whole body metabolism, including energy harvest ${ }^{82}$, intestinal gluconeogenesis (i.e. propionate) ${ }^{147}$ and secretion of satiety hormones (GLP-1, PYY) from enteroendocrine cells ${ }^{148,149}$. Furthermore, SCFA may exert tissue specific effects that can ameliorate an insulin resistant state and aid in whole-body energy homeostasis and weight management. Several studies have reported an antilipolytic effect of acetate in animal adipocytes ${ }^{128,150,151}$, and on whole body level in animals ${ }^{150,152,153}$ and humans ${ }^{154-157}$. In contrast, butyrate and propionate have been shown to both enhance ${ }^{158}$ and attenuate lipolysis (basal and TNF $\alpha$ stimulated) ${ }^{150,159}$. In the adipose tissue, SCFA have been proposed to signal through the GPR41/43 receptors and attenuate the phosphorylation of hormone sensitive lipase (HSL at serine 563). Of note, mechanistic studies have been performed in murine adipose cells (which may lack GPR41) ${ }^{160}$ with incubations of single SCFA using supraphysiological concentrations (4-10 mmol/L). This calls for future studies that investigate SCFA effects in human adipocyte models using physiological relevant SCFA concentrations. Additionally, SCFA may also improve adipose tissue function and remodeling through increased adipogenesis, expression of browning markers (i.e. Uncoupling protein-1) and alleviation of an inflammatory state through improvements in circulating proinflammatory cytokine profiles 36,161 .

Furthermore, circulating SCFA may directly reach and modulate skeletal muscle metabolism through increases in AMP as previously observed in murine myotubes 162 and in vivo in skeletal muscle of animals ${ }^{63,163}$. For example, according to an in vitro study using L6 myotubes, acetic acid increased AMP/ATP ratio and AMPK (threonine 172) phosphorylation in a dose-dependent (0.05-0.5 $\mathrm{mmol} / \mathrm{L}$ ) and time-dependent (0-30 $\mathrm{min})$ manner using physiological acetic acid concentrations ${ }^{164}$. In line, muscle gastrocnemius extracts from butyrate- 
supplemented mice (C57BL/6J) showed increments in radiolabeled palmitate oxidation by $200 \%{ }^{162}$. In addition, acetate intragastric infusions (6 months) ${ }^{165}$, and in vitro acetate incubations using L6 myotubes $(0.05-0.5 \mathrm{mmol} / \mathrm{L}){ }^{164}$, both showed upregulation of glucose transporter 4 (GLUT4). Of note, these findings show the potential of SCFA to increase fat oxidation and/or glucose uptake, which warrants further investigation in humans. In rat hepatocytes, incubations with acetate (0.1-0.2 mmol/L) increased phosphorylation of AMPK (threonine 172 ) and fat oxidation ${ }^{166}$. The liver, as explained above is in close proximity with the proximal colon and gut-derived SCFA may modulate hepatic processes including lipogenesis and cholesterol biosynthesis (mainly acetate) and gluconeogenesis (propionate) ${ }^{100-102,167}$. Importantly, SCFA may also promote gut integrity through the upregulation of tight junction proteins ${ }^{168}$ and preservation of a healthy mucosa that decreases translocation of proinflammatory molecules (i.e. lipopolysaccharides, LPS) 169 to the liver and the systemic circulation (metabolic endotoxemia).

Circulating SCFA may also modulate pancreatic functioning ${ }^{170}$. In support, SCFA have been shown in animal and in vitro studies (using isolated human islets) to modulate glucose-stimulated insulin secretion, however, their role and physiological relevance in pancreatic insulin secretion in humans

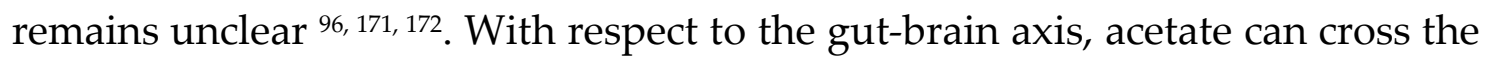
blood brain barrier in animals ${ }^{173}$ and humans ${ }^{174}$ and together with SCFAinduced secretion of gut-derived hormones (GLP-1, PYY) regulate satietyinducing effects through central hypothalamic activation (vagal activation) ${ }^{33}$. In summary, colonic SCFA may improve gut health and may reach the circulation in the aforementioned tissues, thereby directly/indirectly modulating substrate and energy metabolism (See Figure 1). In the next section, we discuss the role of SCFA in weight management and insulin sensitivity in humans. 


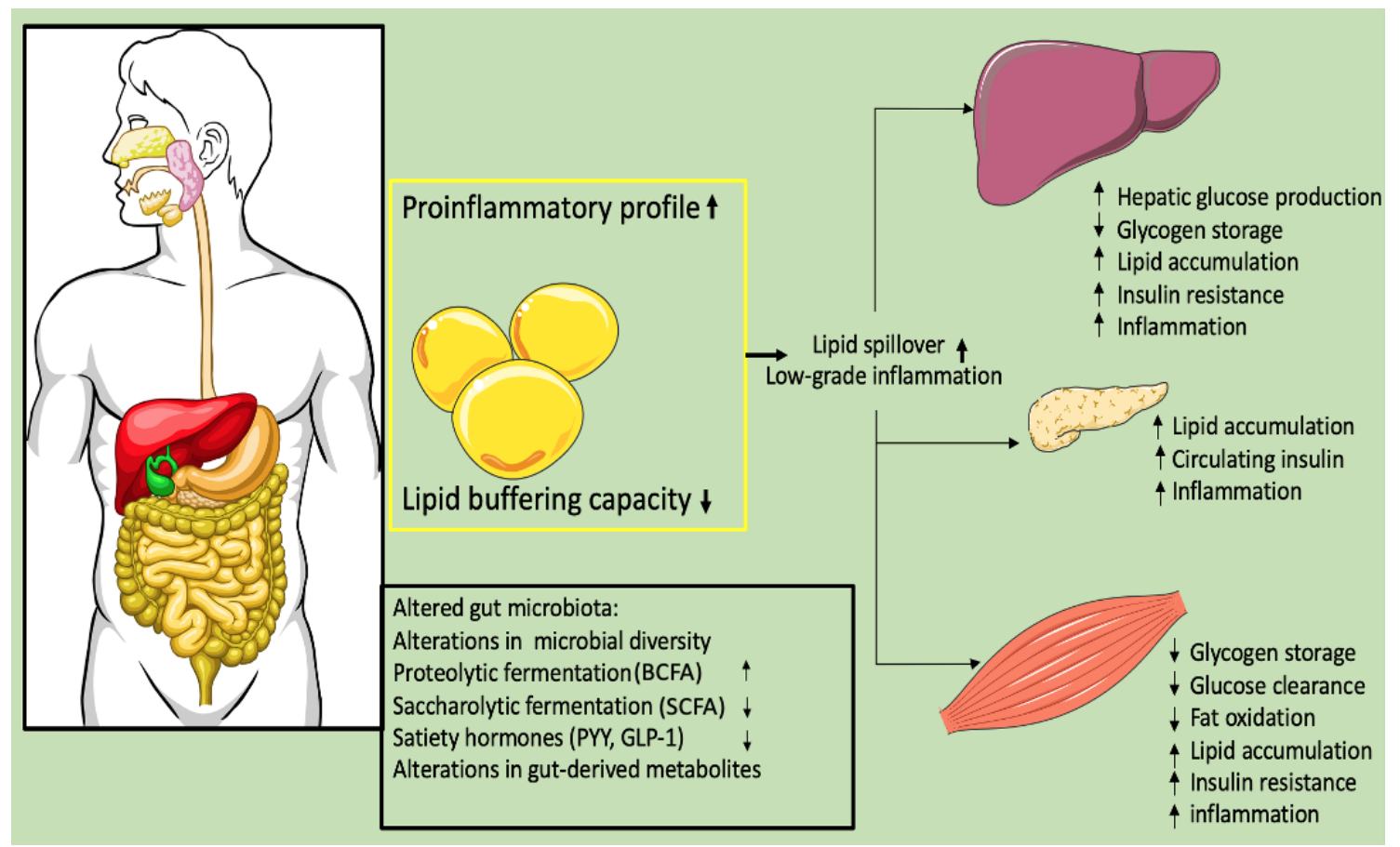

Figure 1. Interorgan crosstalk in an insulin resistant state. Adiposity impairs the adipocyte lipid buffering capacity, thereby releasing circulating lipids in the circulation (lipid spillover). The resulting lipid accumulation in the liver and skeletal muscle may impair insulin signaling, decrease glycogen storage, increase hepatic glucose output and may result in hyperglycemia. Additionally, adipose tissue dysfunction can lead to proinflammatory adipokine/cytokine secretion, which results in a low-grade inflammation state that disturbs insulin signaling. In the gut, altered microbial composition, diversity and functionality, altered balance between saccharolytic and proteolytic fermentation as well as altered SCFA and BCFA concentrations may impact gut and metabolic health. In light of the current literature, a high fiber diet to promote a high SCFA/BCFA ratio may be preferred to improve gut health, functioning in the aforementioned tissues and whole-body insulin sensitivity. However, more information is needed on plasma SCFA relevance at the tissue level as well as on whole-body substrate metabolism and insulin sensitivity in humans.

\section{SCFA in control of body weight and insulin sensitivity}

Limited human studies have investigated SCFA effects on parameters of body weight control and insulin sensitivity. One study showed that oral administration of sodium propionate increased fasting fat oxidation and resting energy expenditure independent of glucose and insulin levels in healthy subjects 175. In line, distal colonic administration of SCFA mixtures and acetate alone resulted in increments in fasting fat oxidation, resting energy expenditure as well as in fasting PYY levels in overweight or obese individuals ${ }^{91,92}$. However, these effects were not observed after proximal colonic acetate infusions.

As described above, a higher distal colonic SCFA release compared to the proximal colon points towards a relevance of distal colonic SCFA production in metabolic health. SCFA may also increase fat oxidation and energy expenditure indirectly through their effects on gut hormone production (i.e. GLP-1 and PYY) as observed in humans ${ }^{176}$. Recently, we showed that long-term supplementation with galacto-oligosaccharides (12-week, 15g/d, GOS), an acetogenic fiber, had no significant effect on peripheral and adipose tissue insulin sensitivity as well as 
no effects on fecal and circulating acetate and SCFA despite an observed bifidogenic effect ${ }^{34}$. In view of our above described findings that acute distal colonic SCFA infusions, but not proximal colonic affected parameters of metabolic health ${ }^{91}$, we hypothesized that the site of fermentation (distal versus proximal) of GOS may have led to an insufficient increase in systemic SCFA to induce pronounced metabolic effects. Thus, increasing dietary fibers that stimulate SCFA production in the distal colon may have the most pronounced effects on parameters of metabolic health. Interestingly, several cohort studies and prebiotic interventions in humans have shown improvements in markers of insulin sensitivity ${ }^{177-180}$. For instance, one study (a 4-week intervention using resistant starch) in healthy subjects showed increments in both skeletal muscle and adipose tissue uptake of SCFA (acetate and propionate) together with improved insulin sensitivity (measured via the hyperinsulinemic-euglycemic clamp) ${ }^{178,179}$, indicating that gut-derived SCFA from saccharolytic fermentation may reach the systemic circulation and insulin sensitive tissues. Notably, a recent study from our group investigated acute metabolic effects of the naturallyoccurring soluble fiber inulin and using stable isotope tracer methodology to trace inulin-derived SCFA ${ }^{35}$. Inulin increased fermentation in the early postprandial phase $\left({ }^{13} \mathrm{CO}_{2}\right)$, increased circulating acetate in late postprandial phase (3-7 hrs) and fat oxidation in the early postprandial phase (0-3 hrs) in overweight-obese individuals ${ }^{35}$.

Furthermore, probiotic supplementation of certain microbial strains (Akkermansia muciniphila) may promote a healthy gut functioning as well as improvements in metabolic health ${ }^{181-184}$. For instance, a recent probiotic supplementation for 3-months using living and pasteurized Akkermansia muciniphila in overweight-obese insulin resistant volunteers showed improvements in insulin sensitivity (Homeostatic Model Assessment of Insulin Resistance (HOMA-IR)) ${ }^{185}$.

Of interest, this long-term supplementation was safe and well tolerated. Although the exact mechanisms were not explored in detail (some effects were reductions in total cholesterol and white blood cells), it is reasonable to hypothesize that Akkermansia muciniphila, being an acetate producer, may result in elevated acetate and acetate-mediated metabolic effects in the host. In chapter 2 , we extensively reviewed the role of acetate in body weight control and insulin sensitivity ${ }^{33}$. In particular acetate, the most abundant SCFA, seems to be of utmost importance in prebiotic/probiotic effects on metabolism, as also evident from positive metabolic effects reported with vinegar (which contains as primary bioactive compound acetic acid/acetate $5-10 \%$ per volume) administrations and the above-described distal colonic acetate infusions ${ }^{91,92,186}$.

Lastly, the effectiveness/outcome of studies focusing on modulating SCFA production or metabolism may depend on the individual metabolic (possibly differing between healthy versus metabolically disturbed individuals) 187 or microbial phenotype ${ }^{188}$. For instance, a study showed that oral supplementation of butyrate improved glucose metabolism in lean, but not in 
individuals with characteristics of the metabolic syndrome ${ }^{189}$. In line, vinegar supplementation studies have been shown to beneficially affect glucose homeostasis in healthy individuals, but to a lesser extent in individuals with impaired glucose tolerance ${ }^{186}$. With respect to microbial phenotype, it should be considered that modulation of microbial composition might depend on microbial enterotypes, which are three distinct robust clusters that can stratify microbial community (Bacteroides, Prevotella, Ruminococcus) 188, 190, 191. Furthermore, microbial composition may impact insulin sensitivity in a sexspecific manner, as a study reported an association in men (but not in women) between Bacteroidetes/Firmicutes ratio and peripheral insulin sensitivity ${ }^{67}$.

As mentioned above, SCFA may be produced after saccharolytic (i.e. inulin, fructo-oligosaccharides, GOS) fermentation 26, 192, 193 and BCFA after proteolytic (BCAA, leucine, isoleucine and valine) fermentation ${ }^{25,29}$ and linked to beneficial and detrimental effects on metabolic health, respectively. Under this premise, it has been postulated that a balance in carbohydrate/protein fermentation may be of importance for metabolic health ${ }^{25}$. In general, an animalbased diet has higher digestibility and net protein utilization as compared to a plant-based diet ${ }^{194}$. However, little is known about the contribution of a high protein diet varying in animal versus plant-based sources to microbial BCFA production in humans. In various studies with individuals of different phenotypes (BMI 19-51 kg/m²), high protein diet (mixed sources + casein/soy protein) interventions (1-4 weeks), have shown increments in fecal levels of BCFA (isovalerate and isobutyrate) and reductions in fecal levels of SCFA ${ }^{195}$. Although, the literature is relatively vast on SCFA and scarce on BCFA metabolism, human studies are lacking to fully elucidate their role in metabolic health.

Taken together, in vivo human studies investigating SCFA metabolism may need to consider various factors including a) the site of saccharolytic fermentation since this may affect metabolic outcome with more pronounced metabolic effects with distal colonic fermentation, b) the intervention success may depend on metabolic phenotype, as explained above, c) sex-specific effects of gut-derived metabolites since microbial composition relates in a sex-specific manner with peripheral insulin sensitivity. Furthermore, a vast wealth of literature comes from animal data, and studies addressing tissue specific (adipose tissue, skeletal muscle) effects in human cell models are lacking.

In the next section we discuss the role of BCFA in weight management and insulin sensitivity in the interorgan crosstalk. 


\section{Role of BCFA in the interorgan crosstalk, body weight control and insulin sensitivity}

Most studies have investigated fecal concentrations of BCFA (isovalerate and isobutyrate), and only a few investigated their role in metabolic health and insulin sensitivity. A germ-free mouse model fed a high fat diet showed development of NAFLD and insulin resistance together with high levels of isobutyrate and isovalerate in the cecum ${ }^{196}$. Similarly, individuals with obesity have reported higher fecal BCFA concentrations as compared to healthy individuals ${ }^{129}$ as well as association of fecal BCFA with the development of NAFLD in obese individuals ${ }^{197}$. Additionally, fecal BCFA in humans have reported higher abundance in individuals with hypercholesterolemia as compared to healthy controls ${ }^{198}$. Interestingly, isobutyrate and isovalerate in the systemic circulation may have different roles in obesity as a study found that plasma isobutyrate concentrations were lower and isovalerate concentrations higher in individuals with obesity compared to lean individuals $(0.33,1.37$ and $0.47,0.25 \mu \mathrm{mol} / \mathrm{L}$, respectively) ${ }^{199}$. Of note, a study using a randomized crossover design showed that a high fiber diet (resistant starch type 2 and arabinoxylan fibers) versus a Western diet in individuals with characteristics of metabolic syndrome reduced fecal BCFA (isobutyrate and isovalerate) and increased fecal SCFA (acetate and butyrate) ${ }^{200}$. Although this indicated a modulation in saccharolytic/proteolytic fermentation ratio, further metabolic effects were not explored. In the circulation, differences in artery/portal vein BCFA concentrations, as measured in individuals during emergency surgeries (described above), suggest net production from the gut ${ }^{123}$. Moreover, based on BCFA receptor expression profile in various tissues (explained above), both isobutyrate and isovalerate have the potential to exert pivotal functions in peripheral tissues. In general, little is known about the role of fecal BCFA (isobutyrate and isovalerate) in human obesity and insulin sensitivity. Nevertheless, there are indications that BCFA production and handling may be disturbed and/or contribute to the progression of insulin resistance and NAFLD. Importantly, high fiber diets may modulate saccharolytic/proteolytic fermentation ratio in metabolically disturbed individuals, which might result in benefits for the metabolic profile of the individual. 


\section{Aim and outline of the thesis}

This doctoral thesis focuses on the role of gut-derived metabolites (SCFA and BCFA) in in vivo metabolic health in human studies with overweight and obese individuals as well as the role of these microbial metabolites in human derived adipose and skeletal muscle tissues in in vitro models.

In chapter 2, we provide a comprehensive review of the current literature on the role of the colonically and systemically most abundant SCFA acetate in body weight control and insulin sensitivity. Here, we discuss acetate effects on satiety and energy expenditure, peripheral tissue metabolism (liver, skeletal muscle, adipose tissue and pancreatic beta cells) and address human intervention studies that modulate acetate ingestion or production (vinegar, preand probiotic). Lastly, we describe that the effect of acetate on metabolic health may depend on the colonic site of production or administration (proximal and distal) and metabolic phenotype.

In light of the vast literature on acetate and its beneficial effects on metabolic health (satiety, energy expenditure, substrate utilization and insulin sensitivity) we hypothesized that there is a positive association between acetate and insulin sensitivity. Thus, we investigated in chapter 3 the associations between sex-specific concentrations of acetate with insulin sensitivity/resistance indices (HOMA-IR, circulating insulin and Matsuda index) in 478 participants (BMI $>27 \mathrm{~kg} / \mathrm{m}^{2}$ ) of the Diet, Obesity and Genes (DiOGenes) Dietary study before and after a low-calorie diet (LCD, $800 \mathrm{kcal} / \mathrm{d}$ ).

Human studies investigating the role of SCFA in metabolic health have predominantly measured fecal levels as a biomarker of SCFA production while only a few have measured circulating SCFA. Therefore, in chapter 4, we investigated the associations between fecal and plasma SCFA (acetate, propionate and butyrate) with metabolic health markers including circulating metabolites, gut hormones, substrate oxidation, inflammatory markers and markers of insulin sensitivity in a well-phenotyped group with a large range in BMI with a normal and an impaired glucose metabolism. In a subgroup, we investigated the fecal and plasma SCFA associations with insulin sensitivity measured via the gold standard hyperinsulinemic-euglycemic clamp technique.

Besides saccharolytic fermentation and SCFA production, proteolytic fermentation may yield various metabolites such as BCFA, which role in human metabolism remains largely unclear. Therefore, in chapter 5 , we investigated the associations of fecal BCFA (isobutyrate and isovalerate) and other fecal metabolites (valerate, lactate, succinate and caproate) with circulating metabolites, substrate oxidation, and markers of insulin sensitivity (circulating insulin and HOMA-IR) in insulin sensitive and insulin resistant individuals. 
A previously conducted in vivo study showed that distal colonic infusions of SCFA (rich in acetate) increased whole-body fasting fat oxidation in men with overweight/obesity ${ }^{91},{ }^{92}$. To elucidate the underlying mechanisms, in chapter 6 , we investigated the effects of sodium acetate (SA) on fat oxidation in human primary muscle cells (HSkMC) derived from a healthy insulin sensitive donor. We investigated the dose and time effect of SA on complete and incomplete endogenous and exogenous oxidation of ${ }^{14} \mathrm{C}$-labelled palmitate as well as SA effect on AMP-activated protein kinase phosphorylation. SCFA have been shown to exert antilipolytic effects in humans, which may reduce lipid overflow ultimately resulting in positive effects on insulin sensitivity. In chapter 7, we used the human multipotent adipose-derived stem (hMADS) cell model to investigate the antilipolytic effect of SCFA and to elucidate the underlying mechanisms. We investigated the in vitro effects of incubation with SCFA mixtures and single SCFA on fasting and stimulated cytosolic lipolysis. Additionally, we investigated the involvement of GPR41/43 receptors and hormone sensitive lipase. In chapter 8, we integrate and discuss the main findings of these studies that investigated the associations/mechanisms of SCFA and BCFA in metabolic health. Finally, we address future perspectives and suggestions for future research. 


\section{References}

1. Engin A. The Definition and Prevalence of Obesity and Metabolic Syndrome. Adv Exp Med Biol. 2017;960:1-17. Epub 2017/06/07. doi: 10.1007/9783-319-48382-5_1. PubMed PMID: 28585193.

2. Finkelstein EA, Khavjou OA, Thompson H, Trogdon JG, Pan L, Sherry B, et al. Obesity and Severe Obesity Forecasts Through 2030. American Journal of Preventive Medicine. 2012;42(6):563-70. doi: 10.1016/j.amepre.2011.10.026.

3. Kok P, Seidell JC, Meinders AE. [The value and limitations of the body mass index (BMI) in the assessment of the health risks of overweight and obesity]. Ned Tijdschr Geneeskd. 2004;148(48):2379-82. Epub 2004/12/24. PubMed PMID: 15615272.

4. Khaodhiar L, McCowen KC, Blackburn GL. Obesity and its comorbid conditions. Clin Cornerstone. 1999;2(3):17-31. Epub 2000/03/04. PubMed PMID: 10696282.

5. Stone TW, McPherson M, Gail Darlington L. Obesity and Cancer: Existing and New Hypotheses for a Causal Connection. EBioMedicine. 2018;30:14-28. Epub 2018/03/13. doi: 10.1016/j.ebiom.2018.02.022. PubMed PMID: 29526577; PubMed Central PMCID: PMCPMC5952217.

6. Lake A, Townshend T. Obesogenic environments: exploring the built and food environments. J R Soc Promot Health. 2006;126(6):262-7. Epub 2006/12/13. PubMed PMID: 17152319.

7. Lakerveld J, Mackenbach J. The Upstream Determinants of Adult Obesity. Obes Facts. 2017;10(3):216-22. Epub 2017/06/01. doi: 10.1159/000471489. PubMed PMID: 28564658; PubMed Central PMCID: PMCPMC5644962.

8. Meldrum DR, Morris MA, Gambone JC. Obesity pandemic: causes, consequences, and solutions-but do we have the will? Fertil Steril. 2017;107(4):833-9. Epub 2017/03/16. doi: 10.1016/j.fertnstert.2017.02.104. PubMed PMID: 28292617.

9. Tremmel M, Gerdtham U-G, Nilsson PM, Saha S. Economic Burden of Obesity: A Systematic Literature Review. Int J Environ Res Public Health. 2017;14(4):435. doi: 10.3390/ijerph14040435. PubMed PMID: 28422077.

10. Durrer Schutz D, Busetto L, Dicker D, Farpour-Lambert N, Pryke R, Toplak H, et al. European Practical and Patient-Centred Guidelines for Adult Obesity Management in Primary Care. Obesity Facts. 2019;12(1):40-66. doi: 10.1159/000496183.

11. Wing RR, Phelan S. Long-term weight loss maintenance. The American Journal of Clinical Nutrition. 2005;82(1):222S-5S. doi: 10.1093/ajcn/82.1.222S.

12. Funk MD, Lee M, Vidoni ML, Reininger BM. Weight loss and weight gain among participants in a community-based weight loss Challenge. BMC Obesity. 2019;6(1):2. doi: 10.1186/s40608-018-0225-1.

13. Kahan S, Manson JE. Obesity Treatment, Beyond the Guidelines: Practical Suggestions for Clinical PracticePractical Suggestions for Obesity Management 
in Clinical PracticePractical Suggestions for Obesity Management in Clinical Practice. JAMA. 2019;321(14):1349-50. doi: 10.1001/jama.2019.2352.

14. Antognoli EL, Seeholzer EL, Gullett H, Jackson B, Smith S, Flocke SA. Primary Care Resident Training for Obesity, Nutrition, and Physical Activity Counseling: A Mixed-Methods Study. Health Promot Pract. 2017;18(5):672-80. Epub 2016/07/13. doi: 10.1177/1524839916658025. PubMed PMID: 27402722.

15. Faith MS, Fontaine KR, Cheskin LJ, Allison DB. Behavioral approaches to the problems of obesity. Behav Modif. 2000;24(4):459-93. Epub 2000/09/19. doi: 10.1177/0145445500244001. PubMed PMID: 10992608.

16. Beck AT, Weishaar M. Cognitive Therapy. In: Freeman A, Simon KM, Beutler LE, Arkowitz H, editors. Comprehensive Handbook of Cognitive Therapy. New York, NY: Springer US; 1989. p. 21-36.

17. Hall H, Perelman D, Breschi A, Limcaoco P, Kellogg R, McLaughlin T, et al. Glucotypes reveal new patterns of glucose dysregulation. PLOS Biology. 2018;16(7):e2005143. doi: 10.1371/journal.pbio.2005143.

18. Zeevi D, Korem T, Zmora N, Israeli D, Rothschild D, Weinberger A, et al. Personalized Nutrition by Prediction of Glycemic Responses. Cell. 2015;163(5):1079-94. Epub 2015/11/23. doi: 10.1016/j.cell.2015.11.001. PubMed PMID: 26590418.

19. Trouwborst I, Bowser SM, Goossens GH, Blaak EE. Ectopic Fat Accumulation in Distinct Insulin Resistant Phenotypes; Targets for Personalized Nutritional Interventions. Front Nutr. 2018;5:77-. doi: 10.3389/fnut.2018.00077. PubMed PMID: 30234122.

20. Raynor HA, Champagne CM. Position of the Academy of Nutrition and Dietetics: Interventions for the Treatment of Overweight and Obesity in Adults. J Acad Nutr Diet. 2016;116(1):129-47. Epub 2016/01/01. doi: 10.1016/j.jand.2015.10.031. PubMed PMID: 26718656.

21. Cani PD. Human gut microbiome: hopes, threats and promises. Gut. 2018;67(9):1716. doi: 10.1136/gutjnl-2018-316723.

22. Walter J, Armet AM, Finlay BB, Shanahan F. Establishing or Exaggerating Causality for the Gut Microbiome: Lessons from Human Microbiota-Associated Rodents. Cell. 2020;180(2):221-32. doi: 10.1016/j.cell.2019.12.025.

23. Bleau C, Karelis AD, St-Pierre DH, Lamontagne L. Crosstalk between intestinal microbiota, adipose tissue and skeletal muscle as an early event in systemic low-grade inflammation and the development of obesity and diabetes. Diabetes Metab Res Rev. 2015;31(6):545-61. Epub 2014/10/30. doi: 10.1002/dmrr.2617. PubMed PMID: 25352002.

24. Ursell LK, Metcalf JL, Parfrey LW, Knight R. Defining the human microbiome. Nutr Rev. 2012;70 Suppl 1(Suppl 1):S38-S44. doi: 10.1111/j.17534887.2012.00493.x. PubMed PMID: 22861806.

25. Canfora EE, Meex RCR, Venema K, Blaak EE. Gut microbial metabolites in obesity, NAFLD and T2DM. Nature Reviews Endocrinology. 2019;15(5):26173. doi: 10.1038/s41574-019-0156-z. 
26. Canfora EE, Jocken JW, Blaak EE. Short-chain fatty acids in control of body weight and insulin sensitivity. Nat Rev Endocrinol. 2015;11(10):577-91. doi: 10.1038/nrendo.2015.128.

27. Koh A, De Vadder F, Kovatcheva-Datchary P, Bäckhed F. From Dietary Fiber to Host Physiology: Short-Chain Fatty Acids as Key Bacterial Metabolites. Cell. 2016;165(6):1332-45. doi: 10.1016/j.cell.2016.05.041.

28. Cummings JH, Pomare EW, Branch WJ, Naylor CP, Macfarlane GT. Short chain fatty acids in human large intestine, portal, hepatic and venous blood. Gut. 1987;28(10):1221-7. doi: 10.1136/gut.28.10.1221. PubMed PMID: 3678950.

29. Mika A, Stepnowski P, Kaska L, Proczko M, Wisniewski P, Sledzinski M, et al. A comprehensive study of serum odd- and branched-chain fatty acids in patients with excess weight. Obesity (Silver Spring). 2016;24(8):1669-76. Epub 2016/06/30. doi: 10.1002/oby.21560. PubMed PMID: 27355152.

30. Ran-Ressler RR, Bae S, Lawrence P, Wang DH, Brenna JT. Branched-chain fatty acid content of foods and estimated intake in the USA. Br J Nutr. 2014;112(4):565-72. Epub 2014/05/17. doi: 10.1017/s0007114514001081. PubMed PMID: 24830474; PubMed Central PMCID: PMCPMC4381348.

31. Russell WR, Gratz SW, Duncan SH, Holtrop G, Ince J, Scobbie L, et al. High-protein, reduced-carbohydrate weight-loss diets promote metabolite profiles likely to be detrimental to colonic health. The American Journal of Clinical Nutrition. 2011;93(5):1062-72. doi: 10.3945/ajcn.110.002188.

32. Rutayisire E, Huang K, Liu Y, Tao F. The mode of delivery affects the diversity and colonization pattern of the gut microbiota during the first year of infants' life: a systematic review. BMC Gastroenterol. 2016;16(1):86. Epub 2016/08/01. doi: 10.1186/s12876-016-0498-0. PubMed PMID: 27475754; PubMed Central PMCID: PMCPMC4967522.

33. Hernandez MAG, Canfora EE, Jocken JWE, Blaak EE. The Short-Chain Fatty Acid Acetate in Body Weight Control and Insulin Sensitivity. Nutrients. 2019;11(8). Epub 2019/08/21. doi: 10.3390/nu11081943. PubMed PMID: 31426593; PubMed Central PMCID: PMCPMC6723943.

34. Canfora EE, van der Beek CM, Hermes GDA, Goossens GH, Jocken JWE, Holst JJ, et al. Supplementation of Diet With Galacto-oligosaccharides Increases Bifidobacteria, but Not Insulin Sensitivity, in Obese Prediabetic Individuals. Gastroenterology. 2017;153(1):87-97.e3. Epub 2017/04/12. doi: 10.1053/j.gastro.2017.03.051. PubMed PMID: 28396144.

35. van der Beek CM, Canfora EE, Kip AM, Gorissen SHM, Olde Damink SWM, van Eijk HM, et al. The prebiotic inulin improves substrate metabolism and promotes short-chain fatty acid production in overweight to obese men. Metabolism. 2018;87:25-35. Epub 2018/06/29. doi: 10.1016/j.metabol.2018.06.009. PubMed PMID: 29953876.

36. Canfora EE, Jocken JW, Blaak EE. Short-chain fatty acids in control of body weight and insulin sensitivity. Nat Rev Endocrinol. 2015;11(10):577-91. Epub 2015/08/12. doi: 10.1038/nrendo.2015.128. PubMed PMID: 26260141. 
37. Reijnders D, Goossens GH, Hermes GD, Neis EP, van der Beek CM, Most J, et al. Effects of Gut Microbiota Manipulation by Antibiotics on Host Metabolism in Obese Humans: A Randomized Double-Blind Placebo-Controlled Trial. Cell metabolism. 2016;24(1):63-74. Epub 2016/07/15. doi: 10.1016/j.cmet.2016.06.016. PubMed PMID: 27411009.

38. Tasnim N, Abulizi N, Pither J, Hart MM, Gibson DL. Linking the Gut Microbial Ecosystem with the Environment: Does Gut Health Depend on Where We Live? Front Microbiol. 2017;8:1935-. doi: 10.3389/fmicb.2017.01935. PubMed PMID: 29056933.

39. Musso G, Gambino R, Cassader M. Obesity, Diabetes, and Gut Microbiota. Diabetes Care. 2010;33(10):2277. doi: 10.2337/dc10-0556.

40. Hylander BL, Repasky EA. Temperature as a modulator of the gut microbiome: what are the implications and opportunities for thermal medicine? International Journal of Hyperthermia. 2019;36(sup1):83-9. doi: 10.1080/02656736.2019.1647356.

41. Janda JM, Abbott SL. $16 S$ rRNA Gene Sequencing for Bacterial Identification in the Diagnostic Laboratory: Pluses, Perils, and Pitfalls. Journal of Clinical Microbiology. 2007;45(9):2761. doi: 10.1128/JCM.01228-07.

42. Zhang C, Derrien M, Levenez F, Brazeilles R, Ballal SA, Kim J, et al. Ecological robustness of the gut microbiota in response to ingestion of transient food-borne microbes. The ISME Journal. 2016;10(9):2235-45. doi: 10.1038/ismej.2016.13.

43. Kim SW, Suda W, Kim S, Oshima K, Fukuda S, Ohno H, et al. Robustness of gut microbiota of healthy adults in response to probiotic intervention revealed by high-throughput pyrosequencing. DNA Res. 2013;20(3):241-53. Epub 2013/04/11. doi: 10.1093/dnares/dst006. PubMed PMID: 23571675; PubMed Central PMCID: PMCPMC3686430.

44. Koliada A, Syzenko G, Moseiko V, Budovska L, Puchkov K, Perederiy V, et al. Association between body mass index and Firmicutes/Bacteroidetes ratio in an adult Ukrainian population. BMC Microbiol. 2017;17(1):120-. doi: 10.1186/s12866-017-1027-1. PubMed PMID: 28532414.

45. Turnbaugh PJ, Ley RE, Mahowald MA, Magrini V, Mardis ER, Gordon JI. An obesity-associated gut microbiome with increased capacity for energy harvest. Nature. 2006;444(7122):1027-31. doi: 10.1038/nature05414.

46. Ley RE, Bäckhed F, Turnbaugh P, Lozupone CA, Knight RD, Gordon JI. Obesity alters gut microbial ecology. Proceedings of the National Academy of Sciences of the United States of America. 2005;102(31):11070. doi: 10.1073/pnas.0504978102.

47. Castaner O, Goday A, Park Y-M, Lee S-H, Magkos F, Shiow S-ATE, et al. The Gut Microbiome Profile in Obesity: A Systematic Review. Int J Endocrinol. 2018;2018:4095789-. doi: 10.1155/2018/4095789. PubMed PMID: 29849617.

48. Mariat D, Firmesse O, Levenez F, Guimarăes VD, Sokol H, Doré J, et al. The Firmicutes/Bacteroidetes ratio of the human microbiota changes with age. BMC Microbiol. 2009;9(1):123. doi: 10.1186/1471-2180-9-123. 
49. Schwiertz A, Taras D, Schäfer K, Beijer S, Bos NA, Donus C, et al. Microbiota and SCFA in Lean and Overweight Healthy Subjects. Obesity. 2010;18(1):190-5. doi: 10.1038/oby.2009.167.

50. Lee B, Moon KM, Kim CY. Tight Junction in the Intestinal Epithelium: Its Association with Diseases and Regulation by Phytochemicals. J Immunol Res. 2018;2018:2645465. Epub 2019/01/17. doi: 10.1155/2018/2645465. PubMed PMID: 30648119; PubMed Central PMCID: PMCPMC6311762.

51. Sharma S, Tripathi P. Gut microbiome and type 2 diabetes: where we are and where to go? J Nutr Biochem. 2019;63:101-8. Epub 2018/10/27. doi: 10.1016/j.jnutbio.2018.10.003. PubMed PMID: 30366260.

52. Gribble FM, Reimann F. Function and mechanisms of enteroendocrine cells and gut hormones in metabolism. Nature Reviews Endocrinology. 2019;15(4):226-37. doi: 10.1038/s41574-019-0168-8.

53. Belizario JE, Faintuch J. Microbiome and Gut Dysbiosis. Exp Suppl. 2018;109:459-76. Epub 2018/12/12. doi: 10.1007/978-3-319-74932-7_13. PubMed PMID: 30535609.

54. Cotillard A, Kennedy SP, Kong LC, Prifti E, Pons N, Le Chatelier E, et al. Dietary intervention impact on gut microbial gene richness. Nature. 2013;500:585. doi: 10.1038/nature12480.

55. Yasir M, Angelakis E, Bibi F, Azhar EI, Bachar D, Lagier JC, et al. Comparison of the gut microbiota of people in France and Saudi Arabia. Nutr Diabetes. 2015;5:e153. Epub 2015/04/29. doi: 10.1038/nutd.2015.3. PubMed PMID: 25915742; PubMed Central PMCID: PMCPMC4423199.

56. Turnbaugh PJ, Hamady M, Yatsunenko T, Cantarel BL, Duncan A, Ley $\mathrm{RE}$, et al. A core gut microbiome in obese and lean twins. Nature. 2009;457(7228):480-4. doi: 10.1038/nature07540.

57. Lv Y, Qin X, Jia H, Chen S, Sun W, Wang X. The Association between Gut Microbiota Composition and Body Mass Index in Chinese Male College Students, as Analyzed by Next-generation Sequencing. British Journal of Nutrition.1-17. Epub 2019/08/09. doi: 10.1017/S0007114519001909.

58. Rizzatti G, Lopetuso LR, Gibiino G, Binda C, Gasbarrini A. Proteobacteria: A Common Factor in Human Diseases. BioMed Research International. 2017;2017:7. doi: 10.1155/2017/9351507.

59. Schierwagen R, Alvarez-Silva C, Madsen MSA, Kolbe CC, Meyer C, Thomas D, et al. Circulating microbiome in blood of different circulatory compartments. Gut. 2019;68(3):578. doi: 10.1136/gutjnl-2018-316227.

60. Garcia Caraballo SC, Comhair TM, Houten SM, Dejong CH, Lamers WH, Koehler SE. High-protein diets prevent steatosis and induce hepatic accumulation of monomethyl branched-chain fatty acids. J Nutr Biochem. 2014;25(12):1263-74. Epub 2014/10/08. doi: 10.1016/j.jnutbio.2014.07.005. PubMed PMID: 25287814.

61. Dornas W, Lagente V. Intestinally derived bacterial products stimulate development of nonalcoholic steatohepatitis. Pharmacol Res. 2019;141:418-28. Epub 2019/01/19. doi: 10.1016/j.phrs.2019.01.026. PubMed PMID: 30658094. 
62. Houghton MJ, Kerimi A, Mouly V, Tumova S, Williamson G. Gut microbiome catabolites as novel modulators of muscle cell glucose metabolism. Faseb j. 2019;33(2):1887-98. Epub 2018/09/06. doi: 10.1096/fj.201801209R. PubMed PMID: 30183376.

63. Frampton J, Murphy K, Frost G, Chambers E. Short-chain fatty acids as potential regulators of skeletal muscle metabolism and function. Nature Metabolism. 2020. doi: 10.1038/s42255-020-0188-7.

64. Pagliari D, Saviano A, Newton EE, Serricchio ML, Dal Lago AA, Gasbarrini A, et al. Gut Microbiota-Immune System Crosstalk and Pancreatic Disorders. Mediators Inflamm. 2018;2018:7946431-. doi: 10.1155/2018/7946431. PubMed PMID: 29563853.

65. Mandaliya DK, Seshadri S. Short Chain Fatty Acids, pancreatic dysfunction and type 2 diabetes. Pancreatology. 2019;19(2):280-4. doi: 10.1016/j.pan.2019.01.021.

66. Wilmanski T, Rappaport N, Earls JC, Magis AT, Manor O, Lovejoy J, et al. Blood metabolome predicts gut microbiome $\alpha$-diversity in humans. Nature Biotechnology. 2019. doi: 10.1038/s41587-019-0233-9.

67. Most J, Goossens GH, Reijnders D, Canfora EE, Penders J, Blaak EE. Gut microbiota composition strongly correlates to peripheral insulin sensitivity in obese men but not in women. Benef Microbes. 2017;8(4):557-62. Epub 2017/06/18. doi: 10.3920/bm2016.0189. PubMed PMID: 28618864.

68. Qin J, Li Y, Cai Z, Li S, Zhu J, Zhang F, et al. A metagenome-wide association study of gut microbiota in type 2 diabetes. Nature. 2012;490(7418):5560. doi: 10.1038/nature11450.

69. Karlsson FH, Tremaroli V, Nookaew I, Bergström G, Behre CJ, Fagerberg B, et al. Gut metagenome in European women with normal, impaired and diabetic glucose control. Nature. 2013;498(7452):99-103. doi: 10.1038/nature12198.

70. Janssen AW, Kersten S. The role of the gut microbiota in metabolic health. Faseb j. 2015;29(8):3111-23. Epub 2015/04/30. doi: 10.1096/fj.14-269514. PubMed PMID: 25921831.

71. Rivière A, Selak M, Lantin D, Leroy F, De Vuyst L. Bifidobacteria and Butyrate-Producing Colon Bacteria: Importance and Strategies for Their Stimulation in the Human Gut. Front Microbiol. 2016;7:979-. doi: 10.3389/fmicb.2016.00979. PubMed PMID: 27446020.

72. Müller M, Canfora E, Blaak E. O14.3 The effect of long term Arabinoxylanoligosaccharide supplementation on gastrointestinal functioning and metabolic parameters: A randomized controlled trial2018.

73. Jamar G, Santamarina AB, Casagrande BP, Estadella D, de Rosso VV, Wagner R, et al. Prebiotic potencial of juçara berry on changes in gut bacteria and acetate of individuals with obesity. European Journal of Nutrition. 2020. doi: 10.1007/s00394-020-02208-1. 
74. Meyer D, Stasse-Wolthuis M. The bifidogenic effect of inulin and oligofructose and its consequences for gut health. European Journal of Clinical Nutrition. 2009;63(11):1277-89. doi: 10.1038/ejcn.2009.64.

75. Sanders ME, Merenstein DJ, Reid G, Gibson GR, Rastall RA. Probiotics and prebiotics in intestinal health and disease: from biology to the clinic. Nat Rev Gastroenterol Hepatol. 2019. Epub 2019/07/13. doi: 10.1038/s41575-019-0173-3. PubMed PMID: 31296969.

76. Anand S, Kaur H, Mande SS. Comparative In silico Analysis of Butyrate Production Pathways in Gut Commensals and Pathogens. Front Microbiol. 2016;7(1945). doi: 10.3389/fmicb.2016.01945.

77. Besten Gd, Lange K, Havinga R, Dijk THv, Gerding A, Eunen Kv, et al. Gut-derived short-chain fatty acids are vividly assimilated into host carbohydrates and lipids. American Journal of Physiology-Gastrointestinal and Liver Physiology. 2013;305(12):G900-G10. doi: 10.1152/ajpgi.00265.2013. PubMed PMID: 24136789.

78. Kaur A, Rose DJ, Rumpagaporn P, Patterson JA, Hamaker BR. In Vitro Batch Fecal Fermentation Comparison of Gas and Short-Chain Fatty Acid Production Using "Slowly Fermentable" Dietary Fibers. Journal of Food Science. 2011;76(5):H137-H42. doi: 10.1111/j.1750-3841.2011.02172.x.

79. Cummings JH, Macfarlane GT. Colonic microflora: nutrition and health. Nutrition. 1997;13(5):476-8. Epub 1997/05/01. doi: 10.1016/s0899-9007(97)00114-7. PubMed PMID: 9225346.

80. Clausen MR, Mortensen PB. Kinetic studies on the metabolism of shortchain fatty acids and glucose by isolated rat colonocytes. Gastroenterology. 1994;106(2):423-32. Epub 1994/02/01. doi: 10.1016/0016-5085(94)90601-7. PubMed PMID: 8299908.

81. Clausen MR, Mortensen PB. Kinetic studies on colonocyte metabolism of short chain fatty acids and glucose in ulcerative colitis. Gut. 1995;37(5):684-9. Epub 1995/11/01. doi: 10.1136/gut.37.5.684. PubMed PMID: 8549946; PubMed Central PMCID: PMCPMC1382875.

82. Thibault R, Blachier F, Darcy-Vrillon B, de Coppet P, Bourreille A, Segain JP. Butyrate utilization by the colonic mucosa in inflammatory bowel diseases: a transport deficiency. Inflamm Bowel Dis. 2010;16(4):684-95. Epub 2009/09/24. doi: 10.1002/ibd.21108. PubMed PMID: 19774643.

83. Bloemen JG, Venema K, van de Poll MC, Olde Damink SW, Buurman WA, Dejong $\mathrm{CH}$. Short chain fatty acids exchange across the gut and liver in humans measured at surgery. Clin Nutr. 2009;28(6):657-61. doi: 10.1016/j.clnu.2009.05.011. PubMed PMID: 19523724.

84. van der Beek CM, Bloemen JG, van den Broek MA, Lenaerts K, Venema K, Buurman WA, et al. Hepatic Uptake of Rectally Administered Butyrate Prevents an Increase in Systemic Butyrate Concentrations in Humans. J Nutr. 2015;145(9):2019-24. Epub 2015/07/15. doi: 10.3945/jn.115.211193. PubMed PMID: 26156796. 
85. Ruppin H, Bar-Meir S, Soergel KH, Wood CM, Schmitt MG, Jr. Absorption of Short-Chain Fatty Acids by the Colon. Gastroenterology. 1980;78(6):1500-7. doi: 10.1016/S0016-5085(19)30508-6.

86. Ruppin H, Bar-Meir S, Soergel KH, Wood CM, Schmitt MG, Jr. Absorption of short-chain fatty acids by the colon. Gastroenterology. 1980;78(6):1500-7. Epub 1980/06/01. PubMed PMID: 6768637.

87. Ritzhaupt A, Wood IS, Ellis A, Hosie KB, Shirazi-Beechey SP. Identification and characterization of a monocarboxylate transporter (MCT1) in pig and human colon: its potential to transport L-lactate as well as butyrate. J Physiol. 1998;513 ( Pt 3)(Pt 3):719-32. doi: 10.1111/j.1469-7793.1998.719ba.x. PubMed PMID: 9824713.

88. Moschen I, Broer A, Galic S, Lang F, Broer S. Significance of short chain fatty acid transport by members of the monocarboxylate transporter family (MCT). Neurochem Res. 2012;37(11):2562-8. Epub 2012/08/11. doi: 10.1007/s11064-012-0857-3. PubMed PMID: 22878645.

89. Müller M, Hernández MAG, Goossens GH, Reijnders D, Holst JJ, Jocken JWE, et al. Circulating but not faecal short-chain fatty acids are related to insulin sensitivity, lipolysis and GLP-1 concentrations in humans. Scientific Reports. 2019;9(1):12515. doi: 10.1038/s41598-019-48775-0.

90. Neis EPJG, van Eijk HMH, Lenaerts K, Olde Damink SWM, Blaak EE, Dejong CHC, et al. Distal versus proximal intestinal short-chain fatty acid release in man. Gut. 2019;68(4):764. doi: 10.1136/gutjnl-2018-316161.

91. van der Beek CM, Canfora EE, Lenaerts K, Troost FJ, Damink S, Holst JJ, et al. Distal, not proximal, colonic acetate infusions promote fat oxidation and improve metabolic markers in overweight/obese men. Clin Sci (Lond). 2016;130(22):2073-82. Epub 2016/07/22. doi: 10.1042/cs20160263. PubMed PMID: 27439969.

92. Canfora EE, van der Beek CM, Jocken JWE, Goossens GH, Holst JJ, Olde Damink SWM, et al. Colonic infusions of short-chain fatty acid mixtures promote energy metabolism in overweight/obese men: a randomized crossover trial. Sci Rep. 2017;7(1):2360. Epub 2017/05/26. doi: 10.1038/s41598-017-02546-x. PubMed PMID: 28539646; PubMed Central PMCID: PMCPMC5443817.

93. Le Poul E, Loison C, Struyf S, Springael JY, Lannoy V, Decobecq ME, et al. Functional characterization of human receptors for short chain fatty acids and their role in polymorphonuclear cell activation. J Biol Chem. 2003;278(28):254819. Epub 2003/04/25. doi: 10.1074/jbc.M301403200. PubMed PMID: 12711604.

94. Brown AJ, Goldsworthy SM, Barnes AA, Eilert MM, Tcheang L, Daniels D, et al. The Orphan G protein-coupled receptors GPR41 and GPR43 are activated by propionate and other short chain carboxylic acids. J Biol Chem. 2003;278(13):11312-9. Epub 2002/12/24. doi: 10.1074/jbc.M211609200. PubMed PMID: 12496283.

95. Tang C, Ahmed K, Gille A, Lu S, Grone H-J, Tunaru S, et al. Loss of FFA2 and FFA3 increases insulin secretion and improves glucose tolerance in type 2 diabetes. Nat Med. 2015;21(2):173-7. doi: 10.1038/nm.3779. 
96. Priyadarshini M, Villa SR, Fuller M, Wicksteed B, Mackay CR, Alquier T, et al. An Acetate-Specific GPCR, FFAR2, Regulates Insulin Secretion. Molecular Endocrinology. 2015;29(7):1055-66. doi: 10.1210/me.2015-1007.

97. Ballard FJ. Supply and utilization of acetate in mammals. Am J Clin Nutr. 1972;25(8):773-9. Epub 1972/08/01. doi: 10.1093/ajcn/25.8.773. PubMed PMID: 4558368 .

98. Knowles SE, Jarrett IG, Filsell OH, Ballard FJ. Production and utilization of acetate in mammals. Biochem J. 1974;142(2):401-11. Epub 1974/08/01. doi: 10.1042/bj1420401. PubMed PMID: 4441381; PubMed Central PMCID: PMCPMC1168292.

99. Mittendorfer B, Sidossis LS, Walser E, Chinkes DL, Wolfe RR. Regional acetate kinetics and oxidation in human volunteers. Am J Physiol. 1998;274(6):E978-83. Epub 1998/06/05. doi: 10.1152/ajpendo.1998.274.6.E978. PubMed PMID: 9611145.

100. Dalile B, Van Oudenhove L, Vervliet B, Verbeke K. The role of short-chain fatty acids in microbiota-gut-brain communication. Nature Reviews Gastroenterology \& Hepatology. 2019;16(8):461-78. doi: 10.1038/s41575-0190157-3.

101. Weitkunat K, Schumann S, Nickel D, Kappo KA, Petzke KJ, Kipp AP, et al. Importance of propionate for the repression of hepatic lipogenesis and improvement of insulin sensitivity in high-fat diet-induced obesity. Mol Nutr Food Res. 2016;60(12):2611-21. Epub 2016/07/29. doi: 10.1002/mnfr.201600305. PubMed PMID: 27467905; PubMed Central PMCID: PMCPMC5215627.

102. Wolever TM, Fernandes J, Rao AV. Serum acetate:propionate ratio is related to serum cholesterol in men but not women. J Nutr. 1996;126(11):2790-7. Epub 1996/11/01. doi: 10.1093/jn/126.11.2790. PubMed PMID: 8914950.

103. McNeil NI, Cummings JH, James WP. Short chain fatty acid absorption by the human large intestine. Gut. 1978;19(9):819-22. PubMed PMID: PMC1412179.

104. Topping DL, Clifton PM. Short-chain fatty acids and human colonic function: roles of resistant starch and nonstarch polysaccharides. Physiological reviews. 2001;81(3):1031-64. Epub 2001/06/28. doi: 10.1152/physrev.2001.81.3.1031. PubMed PMID: 11427691.

105. Rechkemmer G, Rönnau K, Engelhardt WV. Fermentation of polysaccharides and absorption of short chain fatty acids in the mammalian hindgut. Comparative Biochemistry and Physiology Part A: Physiology. 1988;90(4):563-8. doi: 10.1016/0300-9629(88)90668-8.

106. Xing J, Chen JDZ. Alterations of Gastrointestinal Motility in Obesity. Obesity Research. 2004;12(11):1723-32. doi: 10.1038/oby.2004.213.

107. Wong JMW, de Souza R, Kendall CWC, Emam A, Jenkins DJA. Colonic Health: Fermentation and Short Chain Fatty Acids. Journal of Clinical Gastroenterology. 2006;40(3). 
108. Feng W, Ao H, Peng C. Gut Microbiota, Short-Chain Fatty Acids, and Herbal Medicines. Frontiers in Pharmacology. 2018;9(1354). doi: 10.3389/fphar.2018.01354.

109. Müller M, Canfora EE, Blaak EE. Gastrointestinal Transit Time, Glucose Homeostasis and Metabolic Health: Modulation by Dietary Fibers. Nutrients. 2018;10(3):275. doi: 10.3390/nu10030275. PubMed PMID: 29495569.

110. den Besten G, van Eunen K, Groen AK, Venema K, Reijngoud D-J, Bakker $\mathrm{BM}$. The role of short-chain fatty acids in the interplay between diet, gut microbiota, and host energy metabolism. J Lipid Res. 2013;54(9):2325-40. Epub 2013/07/02. doi: 10.1194/jlr.R036012. PubMed PMID: 23821742.

111. Tang Z-Z, Chen G, Hong Q, Huang S, Smith HM, Shah RD, et al. MultiOmic Analysis of the Microbiome and Metabolome in Healthy Subjects Reveals Microbiome-Dependent Relationships Between Diet and Metabolites. Frontiers in Genetics. 2019;10(454). doi: 10.3389/fgene.2019.00454.

112. Nagpal R, Mainali R, Ahmadi S, Wang S, Singh R, Kavanagh K, et al. Gut microbiome and aging: Physiological and mechanistic insights. Nutr Healthy Aging. 2018;4(4):267-85. doi: 10.3233/NHA-170030. PubMed PMID: 29951588.

113. Zhong H, Penders J, Shi Z, Ren H, Cai K, Fang C, et al. Impact of early events and lifestyle on the gut microbiota and metabolic phenotypes in young school-age children. Microbiome. 2019;7(1):2. doi: 10.1186/s40168-018-0608-z.

114. Peters SG, Pomare EW, Fisher CA. Portal and peripheral blood short chain fatty acid concentrations after caecal lactulose instillation at surgery. Gut. 1992;33(9):1249-52. doi: 10.1136/gut.33.9.1249. PubMed PMID: 1427380.

115. Mortensen PB, Holtug K, Rasmussen HS. Short-Chain Fatty Acid Production from Mono- and Disaccharides in a Fecal Incubation System: Implications for Colonic Fermentation of Dietary Fiber in Humans. The Journal of Nutrition. 1988;118(3):321-5. doi: 10.1093/jn/118.3.321.

116. Smith EA, Macfarlane GT. Enumeration of amino acid fermenting bacteria in the human large intestine: effects of $\mathrm{pH}$ and starch on peptide metabolism and dissimilation of amino acids. FEMS Microbiology Ecology. 1998;25(4):355-68. doi: 10.1111/j.1574-6941.1998.tb00487.x.

117. Gibson JA, Sladen GE, Dawson AM. Protein absorption and ammonia production: the effects of dietary protein and removal of the colon. Br J Nutr. 1976;35(1):61-5. Epub 1976/01/01. doi: 10.1079/bjn19760009. PubMed PMID: 1244844.

118. Silvester KR, Cummings JH. Does digestibility of meat protein help explain large bowel cancer risk? Nutr Cancer. 1995;24(3):279-88. Epub 1995/01/01. doi: 10.1080/01635589509514417. PubMed PMID: 8610047.

119. Ran-Ressler RR, Bae S, Lawrence P, Wang DH, Brenna JT. Branched-chain fatty acid content of foods and estimated intake in the USA. The British journal of nutrition. 2014;112(4):565-72. Epub 2014/05/15. doi: 10.1017/S0007114514001081. PubMed PMID: 24830474.

120. Adamska A, Rutkowska J. [Odd- and branched-chain fatty acids in milk fat--characteristic and health properties]. Postepy Hig Med Dosw (Online). 
2014;68:998-1007. Epub 2014/09/18. doi: 10.5604/17322693.1118188. PubMed PMID: 25228507.

121. Nilsson A, Johansson E, Ekström L, Björck I. Effects of a brown beans evening meal on metabolic risk markers and appetite regulating hormones at a subsequent standardized breakfast: a randomized cross-over study. PLoS One. 2013;8(4):e59985-e. Epub 2013/04/05. doi: 10.1371/journal.pone.0059985. PubMed PMID: 23577078.

122. Cardona ME, Collinder E, Stern S, Tjellström B, Norin E, Midtvedt T. Correlation between faecal iso-butyric and iso-valeric acids in different species. Microbial Ecology in Health and Disease. 2005;17(3):177-82. doi: 10.1080/08910600500356265.

123. Macfarlane GT, Gibson GR, Beatty E, Cummings JH. Estimation of shortchain fatty acid production from protein by human intestinal bacteria based on branched-chain fatty acid measurements. FEMS Microbiology Ecology. 1992;10(2):81-8. doi: 10.1111/j.1574-6941.1992.tb00002.x.

124. Lagkouvardos I, Kläring K, Heinzmann SS, Platz S, Scholz B, Engel K-H, et al. Gut metabolites and bacterial community networks during a pilot intervention study with flaxseeds in healthy adult men. Molecular Nutrition \& Food Research. 2015;59(8):1614-28. doi: 10.1002/mnfr.201500125.

125. Beumer J, Clevers H. How the Gut Feels, Smells, and Talks. Cell. 2017;170(1):10-1. doi: 10.1016/j.cell.2017.06.023.

126. Han YE, Kang CW, Oh JH, Park SH, Ku CR, Cho YH, et al. Olfactory Receptor OR51E1 Mediates GLP-1 Secretion in Human and Rodent Enteroendocrine L Cells. Journal of the Endocrine Society. 2018;2(11):1251-8. doi: 10.1210/js.2018-00165.

127. Xu J, Cheema MU, Pluznick JL. Uncovering the Physiological Role of Olfactory Receptor 558 (Olfr558) in the Vasculature. The FASEB Journal. 2020;34(S1):1-. doi: 10.1096/fasebj.2020.34.s1.03095.

128. Heimann E, Nyman M, Pålbrink A-K, Lindkvist-Petersson K, Degerman E. Branched short-chain fatty acids modulate glucose and lipid metabolism in primary adipocytes. Adipocyte. 2016;5(4):359-68. doi: 10.1080/21623945.2016.1252011. PubMed PMID: 27994949.

129. Tiihonen K, Ouwehand AC, Rautonen N. Effect of overweight on gastrointestinal microbiology and immunology: correlation with blood biomarkers. British Journal of Nutrition. 2010;103(7):1070-8. Epub 2009/11/24. doi: 10.1017/S0007114509992807.

130. Stinkens R, Goossens GH, Jocken JW, Blaak EE. Targeting fatty acid metabolism to improve glucose metabolism. Obes Rev. 2015;16(9):715-57. Epub 2015/07/17. doi: 10.1111/obr.12298. PubMed PMID: 26179344.

131. van Herpen NA, Schrauwen-Hinderling VB. Lipid accumulation in nonadipose tissue and lipotoxicity. Physiol Behav. 2008;94(2):231-41. Epub 2008/01/29. doi: 10.1016/j.physbeh.2007.11.049. PubMed PMID: 18222498. 
132. Summers SA. Ceramides in insulin resistance and lipotoxicity. Prog Lipid Res. 2006;45(1):42-72. Epub 2006/02/01. doi: 10.1016/j.plipres.2005.11.002. PubMed PMID: 16445986.

133. Turpin-Nolan SM, Brüning JC. The role of ceramides in metabolic disorders: when size and localization matters. Nature Reviews Endocrinology. 2020. doi: 10.1038/s41574-020-0320-5.

134. Smith U, Kahn BB. Adipose tissue regulates insulin sensitivity: role of adipogenesis, de novo lipogenesis and novel lipids. J Intern Med. 2016;280(5):465-75. Epub 2016/10/21. doi: 10.1111/joim.12540. PubMed PMID: 27699898; PubMed Central PMCID: PMCPMC5218584.

135. Corpeleijn E, Saris WH, Blaak EE. Metabolic flexibility in the development of insulin resistance and type 2 diabetes: effects of lifestyle. Obes Rev. 2009;10(2):178-93. Epub 2009/02/12. doi: 10.1111/j.1467-789X.2008.00544.x. PubMed PMID: 19207879.

136. Spiegelman BM, Flier JS. Adipogenesis and Obesity: Rounding Out the Big Picture. Cell. 1996;87(3):377-89. doi: 10.1016/S0092-8674(00)81359-8.

137. Virtue S, Vidal-Puig A. Adipose tissue expandability, lipotoxicity and the Metabolic Syndrome--an allostatic perspective. Biochim Biophys Acta. 2010;1801(3):338-49. Epub 2010/01/09. doi: 10.1016/j.bbalip.2009.12.006. PubMed PMID: 20056169.

138. Nishimura S, Manabe I, Nagai R. Adipose tissue inflammation in obesity and metabolic syndrome. Discov Med. 2009;8(41):55-60. Epub 2009/10/01. PubMed PMID: 19788868.

139. Borst SE. The role of TNF-alpha in insulin resistance. Endocrine. 2004;23(2-3):177-82. Epub 2004/05/18. doi: 10.1385/endo:23:2-3:177. PubMed PMID: 15146098.

140. Priest C, Tontonoz P. Inter-organ cross-talk in metabolic syndrome. Nature Metabolism. 2019;1(12):1177-88. doi: 10.1038/s42255-019-0145-5.

141. Yoo HJ, Choi KM. Hepatokines as a Link between Obesity and Cardiovascular Diseases. Diabetes Metab J. 2015;39(1):10-5. Epub 2015/03/03. doi: 10.4093/dmj.2015.39.1.10. PubMed PMID: 25729707; PubMed Central PMCID: PMCPMC4342531.

142. Kodama Y, Brenner DA. c-Jun N-terminal kinase signaling in the pathogenesis of nonalcoholic fatty liver disease: Multiple roles in multiple steps. Hepatology. 2009;49(1):6-8. Epub 2008/12/30. doi: 10.1002/hep.22710. PubMed PMID: 19111006.

143. Lara-Castro C, Garvey WT. Intracellular lipid accumulation in liver and muscle and the insulin resistance syndrome. Endocrinol Metab Clin North Am. 2008;37(4):841-56. doi: 10.1016/j.ecl.2008.09.002. PubMed PMID: 19026935.

144. Kuhlmann J, Neumann-Haefelin C, Belz U, Kalisch J, Juretschke H-P, Stein $\mathrm{M}$, et al. Intramyocellular Lipid and Insulin Resistance. Diabetes. 2003;52(1):138. doi: 10.2337/diabetes.52.1.138.

145. DeFronzo RA, Jacot E, Jequier E, Maeder E, Wahren J, Felber JP. The effect of insulin on the disposal of intravenous glucose. Results from indirect 
calorimetry and hepatic and femoral venous catheterization. Diabetes. 1981;30(12):1000-7. Epub 1981/12/01. doi: 10.2337/diab.30.12.1000. PubMed PMID: 7030826.

146. Gerst F, Wagner R, Oquendo MB, Siegel-Axel D, Fritsche A, Heni M, et al. What role do fat cells play in pancreatic tissue? Mol Metab. 2019;25:1-10. Epub 2019/05/07. doi: 10.1016/j.molmet.2019.05.001. PubMed PMID: 31113756.

147. De Vadder F, Kovatcheva-Datchary P, Goncalves D, Vinera J, Zitoun C, Duchampt A, et al. Microbiota-generated metabolites promote metabolic benefits via gut-brain neural circuits. Cell. 2014;156(1-2):84-96. Epub 2014/01/15. doi: 10.1016/j.cell.2013.12.016. PubMed PMID: 24412651.

148. Cherbut C, Ferrier L, Roze C, Anini Y, Blottiere H, Lecannu G, et al. Shortchain fatty acids modify colonic motility through nerves and polypeptide $\mathrm{YY}$ release in the rat. Am J Physiol. 1998;275(6):G1415-22. Epub 1998/12/09. doi: 10.1152/ajpgi.1998.275.6.G1415. PubMed PMID: 9843779.

149. Silva YP, Bernardi A, Frozza RL. The Role of Short-Chain Fatty Acids From Gut Microbiota in Gut-Brain Communication. Front Endocrinol (Lausanne). 2020;11:25-. doi: 10.3389/fendo.2020.00025. PubMed PMID: 32082260 .

150. Ge H, Li X, Weiszmann J, Wang P, Baribault H, Chen J-L, et al. Activation of G Protein-Coupled Receptor 43 in Adipocytes Leads to Inhibition of Lipolysis and Suppression of Plasma Free Fatty Acids. Endocrinology. 2008;149(9):451926. doi: 10.1210/en.2008-0059.

151. Aberdein N, Schweizer M, Ball D. Sodium acetate decreases phosphorylation of hormone sensitive lipase in isoproterenol-stimulated 3T3-L1 mature adipocytes. Adipocyte. 2014;3(2):121-5. doi: 10.4161/adip.27936. PubMed PMID: PMC3979876.

152. Sahuri-Arisoylu M, Brody LP, Parkinson JR, Parkes H, Navaratnam N, Miller AD, et al. Reprogramming of hepatic fat accumulation and 'browning' of adipose tissue by the short-chain fatty acid acetate. International journal of obesity (2005). 2016;40(6):955-63. Epub 2016/03/16. doi: 10.1038/ijo.2016.23. PubMed PMID: 26975441.

153. Jocken JWE, González Hernández MA, Hoebers NTH, van der Beek CM, Essers YPG, Blaak EE, et al. Short-Chain Fatty Acids Differentially Affect Intracellular Lipolysis in a Human White Adipocyte Model. Front Endocrinol (Lausanne). 2018;8(372). doi: 10.3389/fendo.2017.00372.

154. Crouse JR, Gerson CD, DeCarli LM, Lieber CS. Role of acetate in the reduction of plasma free fatty acids produced by ethanol in man. J Lipid Res. 1968;9(4):509-12.

155. Laurent C, Simoneau C, Marks L, Braschi S, Champ M, Charbonnel B, et al. Effect of acetate and propionate on fasting hepatic glucose production in humans. Eur J Clin Nutr. 1995;49(7):484-91. Epub 1995/07/01. PubMed PMID: 7588498. 
156. Wolever TM, Brighenti F, Royall D, Jenkins AL, Jenkins DJ. Effect of rectal infusion of short chain fatty acids in human subjects. Am J Gastroenterol. 1989;84(9):1027-33. Epub 1989/09/01. PubMed PMID: 2773895.

157. Suokas A, Kupari M, Heikkila J, Lindros K, Ylikahri R. Acute cardiovascular and metabolic effects of acetate in men. Alcoholism, clinical and experimental research. 1988;12(1):52-8. Epub 1988/02/01. PubMed PMID: 3279860.

158. Rumberger JM, Arch JR, Green A. Butyrate and other short-chain fatty acids increase the rate of lipolysis in 3T3-L1 adipocytes. PeerJ. 2014;2:e611. Epub 2014/10/17. doi: 10.7717/peerj.611. PubMed PMID: 25320679; PubMed Central PMCID: PMCPMC4193401.

159. Ohira H, Tsutsui W, Mamoto R, Yamaguchi S, Nishida M, Ito M, et al. Butyrate attenuates lipolysis in adipocytes co-cultured with macrophages through non-prostaglandin E2-mediated and prostaglandin E2-mediated pathways. Lipids Health Dis. 2016;15(1):213-. doi: 10.1186/s12944-016-0387-0. PubMed PMID: 27938411.

160. Inoue D, Tsujimoto G, Kimura I. Regulation of Energy Homeostasis by GPR41. Front Endocrinol (Lausanne). 2014;5(81). doi: 10.3389/fendo.2014.00081. 161. Hong YH, Nishimura Y, Hishikawa D, Tsuzuki H, Miyahara H, Gotoh C, et al. Acetate and propionate short chain fatty acids stimulate adipogenesis via GPCR43. Endocrinology. 2005;146(12):5092-9. Epub 2005/08/27. doi: 10.1210/en.2005-0545. PubMed PMID: 16123168.

162. Gao Z, Yin J, Zhang J, Ward RE, Martin RJ, Lefevre M, et al. Butyrate Improves Insulin Sensitivity and Increases Energy Expenditure in Mice. Diabetes. 2009;58(7):1509. doi: 10.2337/db08-1637.

163. Pan JH, Kim JH, Kim HM, Lee ES, Shin DH, Kim S, et al. Acetic acid enhances endurance capacity of exercise-trained mice by increasing skeletal muscle oxidative properties. Biosci Biotechnol Biochem. 2015;79(9):1535-41. Epub 2015/05/23. doi: 10.1080/09168451.2015.1034652. PubMed PMID: 26000971. 164. Maruta H, Yoshimura Y, Araki A, Kimoto M, Takahashi Y, Yamashita H. Activation of AMP-Activated Protein Kinase and Stimulation of Energy Metabolism by Acetic Acid in L6 Myotube Cells. PLoS One. 2016;11(6):e0158055. doi: 10.1371/journal.pone.0158055.

165. Yamashita H, Maruta H, Jozuka M, Kimura R, Iwabuchi H, Yamato M, et al. Effects of acetate on lipid metabolism in muscles and adipose tissues of type 2 diabetic Otsuka Long-Evans Tokushima Fatty (OLETF) rats. Biosci Biotechnol Biochem. 2009;73(3):570-6. Epub 2009/03/10. doi: 10.1271/bbb.80634. PubMed PMID: 19270372.

166. Sakakibara S, Yamauchi T, Oshima Y, Tsukamoto Y, Kadowaki T. Acetic acid activates hepatic AMPK and reduces hyperglycemia in diabetic KK-A(y) mice. Biochemical and Biophysical Research Communications. 2006;344(2):597604. doi: 10.1016/j.bbrc.2006.03.176.

167. Landau BR, Schumann WC, Chandramouli V, Magnusson I, Kumaran K, Wahren J. 14C-labeled propionate metabolism in vivo and estimates of hepatic 
gluconeogenesis relative to Krebs cycle flux. American Journal of PhysiologyEndocrinology and Metabolism. 1993;265(4):E636-E47. doi: 10.1152/ajpendo.1993.265.4.E636. PubMed PMID: 8238339.

168. Peng L, Li Z-R, Green RS, Holzman IR, Lin J. Butyrate enhances the intestinal barrier by facilitating tight junction assembly via activation of AMPactivated protein kinase in Caco-2 cell monolayers. The Journal of nutrition. 2009;139(9):1619-25. Epub 2009/07/22. doi: 10.3945/jn.109.104638. PubMed PMID: 19625695.

169. Wang F, Liu J, Weng T, Shen K, Chen Z, Yu Y, et al. The Inflammation Induced by Lipopolysaccharide can be Mitigated by Short-chain Fatty Acid, Butyrate, through Upregulation of IL-10 in Septic Shock. Scand J Immunol. 2017;85(4):258-63. Epub 2016/12/13. doi: 10.1111/sji.12515. PubMed PMID: 27943364.

170. Ryu Stacy H, Stappenbeck Thaddeus S. Gut-Pancreatic Axis AMPlified in Islets of Langerhans. Immunity. 2015;43(2):216-8. doi: 10.1016/j.immuni.2015.08.003.

171. Pingitore A, Gonzalez-Abuin N, Ruz-Maldonado I, Huang GC, Frost G, Persaud SJ. Short chain fatty acids stimulate insulin secretion and reduce apoptosis in mouse and human islets in vitro: Role of free fatty acid receptor 2. Diabetes Obes Metab. 2019;21(2):330-9. Epub 2018/09/12. doi: 10.1111/dom.13529. PubMed PMID: 30203438.

172. Orgaard A, Jepsen SL, Holst JJ. Short-chain fatty acids and regulation of pancreatic endocrine secretion in mice. Islets. 2019:1-9. Epub 2019/08/31. doi: 10.1080/19382014.2019.1587976. PubMed PMID: 31469342.

173. Frost G, Sleeth ML, Sahuri-Arisoylu M, Lizarbe B, Cerdan S, Brody L, et al. The short-chain fatty acid acetate reduces appetite via a central homeostatic mechanism. Nat Commun. 2014;5:3611. Epub 2014/05/02. doi: 10.1038/ncomms4611. PubMed PMID: 24781306; PubMed Central PMCID: PMCPMC4015327.

174. Jiang L, Gulanski BI, De Feyter HM, Weinzimer SA, Pittman B, Guidone $\mathrm{E}$, et al. Increased brain uptake and oxidation of acetate in heavy drinkers. J Clin Invest. 2013;123(4):1605-14. Epub 2013/03/08. doi: 10.1172/JCI65153. PubMed PMID: 23478412.

175. Chambers ES, Byrne CS, Aspey K, Chen Y, Khan S, Morrison DJ, et al. Acute oral sodium propionate supplementation raises resting energy expenditure and lipid oxidation in fasted humans. Diabetes Obes Metab. 2018;20(4):1034-9. Epub 2017/11/15. doi: 10.1111/dom.13159. PubMed PMID: 29134744; PubMed Central PMCID: PMCPMC5873405.

176. Pannacciulli N, Bunt JC, Koska J, Bogardus C, Krakoff J. Higher fasting plasma concentrations of glucagon-like peptide 1 are associated with higher resting energy expenditure and fat oxidation rates in humans. Am J Clin Nutr. 2006;84(3):556-60. Epub 2006/09/09. doi: 10.1093/ajcn/84.3.556. PubMed PMID: 16960169. 
177. Weickert MO, Möhlig M, Schöfl C, Arafat AM, Otto B, Viehoff H, et al. Cereal Fiber Improves Whole-Body Insulin Sensitivity in Overweight and Obese Women. Diabetes Care. 2006;29(4):775. doi: 10.2337/diacare.29.04.06.dc05-2374.

178. Robertson MD, Bickerton AS, Dennis AL, Vidal H, Frayn KN. Insulinsensitizing effects of dietary resistant starch and effects on skeletal muscle and adipose tissue metabolism. Am J Clin Nutr. 2005;82(3):559-67. Epub 2005/09/13. doi: 10.1093/ajcn.82.3.559. PubMed PMID: 16155268.

179. Zhao L, Zhang F, Ding X, Wu G, Lam YY, Wang X, et al. Gut bacteria selectively promoted by dietary fibers alleviate type 2 diabetes. Science. 2018;359(6380):1151-6. Epub 2018/03/29. doi: 10.1126/science.aao5774. PubMed PMID: 29590046.

180. Dong Y, Chen L, Gutin B, Zhu H. Total, insoluble, and soluble dietary fiber intake and insulin resistance and blood pressure in adolescents. European Journal of Clinical Nutrition. 2019;73(8):1172-8. doi: 10.1038/s41430-018-0372-y.

181. Belforte FS, Fernandez N, Tonín Monzón F, Rosso AD, Quesada S, Cimolai MC, et al. Getting to Know the Gut Microbial Diversity of Metropolitan Buenos Aires Inhabitants. Front Microbiol. 2019;10(965). doi: 10.3389/fmicb.2019.00965.

182. Naito $\mathrm{Y}$, Uchiyama K, Takagi T. A next-generation beneficial microbe: Akkermansia muciniphila. J Clin Biochem Nutr. 2018;63(1):33-5. Epub 2018/06/20. doi: 10.3164/jcbn.18-57. PubMed PMID: 30087541.

183. Dao MC, Everard A, Aron-Wisnewsky J, Sokolovska N, Prifti E, Verger EO, et al. Akkermansia muciniphila and improved metabolic health during a dietary intervention in obesity: relationship with gut microbiome richness and ecology. Gut. 2016;65(3):426-36. Epub 2015/06/24. doi: 10.1136/gutjnl-2014308778. PubMed PMID: 26100928.

184. Ferreira-Halder CV, Faria AVS, Andrade SS. Action and function of Faecalibacterium prausnitzii in health and disease. Best Pract Res Clin Gastroenterol. 2017;31(6):643-8. Epub 2018/03/24. doi: 10.1016/j.bpg.2017.09.011. PubMed PMID: 29566907.

185. Depommier C, Everard A, Druart C, Plovier H, Van Hul M, Vieira-Silva $S$, et al. Supplementation with Akkermansia muciniphila in overweight and obese human volunteers: a proof-of-concept exploratory study. Nat Med. 2019;25(7):1096-103. Epub 2019/07/03. doi: 10.1038/s41591-019-0495-2. PubMed PMID: 31263284; PubMed Central PMCID: PMCPMC6699990.

186. Lim J, Henry CJ, Haldar S. Vinegar as a functional ingredient to improve postprandial glycemic control-human intervention findings and molecular mechanisms. Mol Nutr Food Res. 2016;60(8):1837-49. Epub 2016/05/24. doi: 10.1002/mnfr.201600121. PubMed PMID: 27213723.

187. Hansen AMB, Wium C, Lee S, Tierney AC, McCarthy D, Roche HM, et al. Substantial inter-individual variations in insulin secretion and sensitivity across the glucometabolic spectrum. Scandinavian Journal of Clinical and Laboratory Investigation. 2020:1-9. doi: 10.1080/00365513.2020.1730433. 
188. Arumugam M, Raes J, Pelletier E, Le Paslier D, Yamada T, Mende DR, et al. Enterotypes of the human gut microbiome. Nature. 2011;473(7346):174-80. doi: 10.1038/nature09944.

189. Bouter K, Bakker GJ, Levin E, Hartstra AV, Kootte RS, Udayappan SD, et al. Differential metabolic effects of oral butyrate treatment in lean versus metabolic syndrome subjects. Clin Transl Gastroenterol. 2018;9(5):155-. doi: 10.1038/s41424-018-0025-4. PubMed PMID: 29799027.

190. Kovatcheva-Datchary P, Nilsson A, Akrami R, Lee YS, De Vadder F, Arora $\mathrm{T}$, et al. Dietary Fiber-Induced Improvement in Glucose Metabolism Is Associated with Increased Abundance of Prevotella. Cell metabolism. 2015;22(6):971-82. Epub 2015/11/11. doi: 10.1016/j.cmet.2015.10.001. PubMed PMID: 26552345.

191. Holscher HD. Dietary fiber and prebiotics and the gastrointestinal microbiota. Gut Microbes. 2017;8(2):172-84. Epub 2017/02/06. doi: 10.1080/19490976.2017.1290756. PubMed PMID: 28165863.

192. Conlon MA, Bird AR. The Impact of Diet and Lifestyle on Gut Microbiota and Human Health. Nutrients. 2015;7(1):17-44. doi: 10.3390/nu7010017. PubMed PMID: PMC4303825.

193. Marchesi JR, Adams DH, Fava F, Hermes GDA, Hirschfield GM, Hold G, et al. The gut microbiota and host health: a new clinical frontier. Gut. 2015.

194. Berrazaga I, Micard V, Gueugneau M, Walrand S. The Role of the Anabolic Properties of Plant- versus Animal-Based Protein Sources in Supporting Muscle Mass Maintenance: A Critical Review. Nutrients. 2019;11(8):1825. doi: 10.3390/nu11081825. PubMed PMID: 31394788.

195. Blachier F, Beaumont M, Portune KJ, Steuer N, Lan A, Audebert M, et al. High-protein diets for weight management: Interactions with the intestinal microbiota and consequences for gut health. A position paper by the my new gut study group. Clinical Nutrition. 2019;38(3):1012-22. doi: 10.1016/j.clnu.2018.09.016.

196. Le Roy T, Llopis M, Lepage P, Bruneau A, Rabot S, Bevilacqua C, et al. Intestinal microbiota determines development of non-alcoholic fatty liver disease in mice. Gut. 2013;62(12):1787-94. Epub 2012/12/01. doi: 10.1136/gutjnl2012-303816. PubMed PMID: 23197411.

197. Da Silva HE, Teterina A, Comelli EM, Taibi A, Arendt BM, Fischer SE, et al. Nonalcoholic fatty liver disease is associated with dysbiosis independent of body mass index and insulin resistance. Sci Rep. 2018;8(1):1466. Epub 2018/01/25. doi: 10.1038/s41598-018-19753-9. PubMed PMID: 29362454; PubMed Central PMCID: PMCPMC5780381.

198. Granado-Serrano AB, Martín-Garí M, Sánchez V, Riart Solans M, Berdún R, Ludwig IA, et al. Faecal bacterial and short-chain fatty acids signature in hypercholesterolemia. Scientific Reports. 2019;9(1):1772. doi: 10.1038/s41598-01938874-3.

199. Aragonès G, Colom-Pellicer M, Aguilar C, Guiu-Jurado E, Martínez S, Sabench F, et al. Circulating microbiota-derived metabolites: a "liquid biopsy? 
International Journal of Obesity. 2020;44(4):875-85. doi: 10.1038/s41366-019-04300 .

200. Hald S, Schioldan AG, Moore ME, Dige A, Lærke HN, Agnholt J, et al. Effects of Arabinoxylan and Resistant Starch on Intestinal Microbiota and ShortChain Fatty Acids in Subjects with Metabolic Syndrome: A Randomised Crossover Study. PLoS One. 2016;11(7):e0159223. Epub 2016/07/21. doi: 10.1371/journal.pone.0159223. PubMed PMID: 27434092; PubMed Central PMCID: PMCPMC4951149. 


\section{CHAPTER 2}

\section{The short-chain fatty acid acetate in body weight control and insulin sensitivity}

Running title: Acetate in body weight control and insulin sensitivity

Manuel A. González Hernández, Emanuel E. Canfora, Johan W.E. Jocken and Ellen E. Blaak

Nutrients. 2019 Aug 18;11(8) 


\begin{abstract}
The interplay of gut microbiota, host metabolism and metabolic health has gained increased attention. Importantly, gut microbiota may play a regulatory role in gastrointestinal health, substrate metabolism and peripheral tissue metabolism including adipose tissue, skeletal muscle, liver, and pancreas via its metabolites short-chain fatty acids (SCFA). Animal and human data demonstrated that, in particular, acetate beneficially affects host energy and substrate metabolism via secretion of the gut hormones like glucagon-like peptide-1 and peptide $Y Y$, thereby affecting appetite, via a reduction in wholebody lipolysis, systemic pro-inflammatory cytokine levels and via an increase in energy expenditure and fat oxidation. Thus, potential therapies to increase gut microbial fermentation and acetate production have been under vigorous scientific scrutiny. In this review, the relevance of the colonically and systemically most abundant SCFA acetate and its effects on the aforementioned tissues will be discussed in relation to body weight control and glucose homeostasis. We discuss in detail the differential effects of oral acetate administration (vinegar intake), colonic acetate infusions, acetogenic fiber and acetogenic probiotic administrations as approaches to combat obesity and comorbidities. Notably, human data are scarce, which highlights the necessity for further human research to investigate acetate's role in host physiology, metabolic and cardiovascular health.
\end{abstract}




\section{Introduction}

Obesity has reached pandemic proportions worldwide, and its increased prevalence is associated with a plethora of metabolic disturbances ${ }^{1}$. The obese state is characterized by increased adipose tissue mass and disturbed function resulting in systemic lipid spillover and low-grade inflammation, which may contribute to the development of comorbidities such as type 2 diabetes mellitus (T2DM) and cardiovascular disease ${ }^{2-4}$. The crosstalk between various metabolic organs such as the gut, liver, adipose tissue and skeletal muscle plays an important regulatory role in energy and substrate metabolism, thereby impacting metabolic health ${ }^{5}$.

In the last decades, the role of the gut microbiota in host energy and substrate metabolism has been under extensive investigation ${ }^{6-8}$. This includes interventions that modify the gut microbiota composition and functionality with antibiotics ${ }^{9,10}$, prebiotics ${ }^{11,12}$, probiotics ${ }^{13}$ and postbiotics ${ }^{14}$. Of note, gut microbes are able to ferment indigestible foods, such as dietary fibers, thereby yielding as end products short-chain fatty acids (SCFA) that may confer beneficial metabolic effects ${ }^{15-17}$. In general, saccharolytic fermentation mostly occurs in the distal ileum and proximal colon. The most abundant SCFA are acetate, propionate and butyrate with an approximate molar ratio of 60:20:20, respectively ${ }^{18-20}$. In mice, the cecum has been described as a major site of SCFA production ${ }^{21}$. After colonic absorption and transition to the systemic circulation, the molar ratio changes to approximately 91:5:4, respectively, numbers that are based on findings in sudden death victims ${ }^{22}$.

Acetate may act through the binding to the G-protein coupled receptors (GPR), GPR43 (FFAR2) and GPR41 (FFAR3), which are expressed at the mRNA and protein level in human colon ${ }^{23,24}$ with variations along the small and large intestine ${ }^{25}$. Moreover, these receptors have been shown to be expressed at the mRNA level in various insulin sensitive tissues such as the adipose tissue ${ }^{26}$, skeletal muscle, liver ${ }^{27}$ and pancreatic beta cells ${ }^{28,29}$, thereby illustrating their broad metabolic role. Intracellularly, although acetate may be converted to acetyl-CoA and incorporated in the tricarboxylic acid (TCA) cycle in various peripheral tissues ${ }^{30-32}$, it may also impact metabolism through increments in oxidative capacity (e. g. liver and skeletal muscle) via effects on 5'AMP-activated protein kinase (AMPK) phosphorylation ${ }^{33-}$ ${ }^{35}$. Of note, acetate may also increase fatty acid synthesis through epigenetic mechanisms such as histone acetylation ${ }^{36}$. Additionally, acetate may activate other receptors important for blood pressure regulation including olfactory receptors 51E2 (Olfr51E2) and 78 (Olfr78) in renal tissue ${ }^{37}$. In this review, we focus on the metabolic effects of the most abundant (in the colon and systemic circulation) gutderived metabolite, acetate, which may improve the obese insulin resistant state through various effects in peripheral tissues that collectively improve body weight control and insulin sensitivity.

We provide an overview of recent literature on dietary sources of acetate, gut-derived acetate production/absorption after fiber fermentation and pre- and probiotics that may increase plasma acetate through colonic fermentation. We will 
discuss the available literature on the effect of acetate on body weight control (central effects of appetite regulation and satiety hormones and energy expenditure), as well as its role in insulin sensitivity in the context of the metabolic inter-organ cross-talk between skeletal muscle, liver, and adipose tissue metabolism ${ }^{38-41}$. Additionally, acetate effects on insulin secretion will be discussed. With respect to acetate effects on cardiovascular health, data is limited and the role of the gut microbiome on cardiovascular health has been reviewed elsewhere ${ }^{42,43}$. Lastly, we discuss potential therapeutic approaches to improve insulin sensitivity and metabolic health, including oral acetate administration (vinegar intake), acetogenic fiber and probiotic supplementations.

\section{Dietary sources and gut-derived acetate production and absorption}

\subsection{Acetate from dietary sources}

According to the Codex General Standard for Food Additives ${ }^{44}$, acetate is present in dietary components as acidity regulator ( $\mathrm{pH}$ control agent), preservative or sequestrant. For instance, acetate-containing foods include dairy products, dried pastas, bread, liquid eggs, salt substitutes, coffee, coffee substitutes, processed meat and smoked/frozen fish ${ }^{44}$. Other important sources are ethanol ${ }^{45}$ and vinegar ${ }^{46}$. Commonly consumed vinegars contain between 4 and $8 \%$ of acetic acid, and vinegar ingestion has gained attention because of its acute effects in glucose and lipid metabolism as extensively reviewed by Lim et al. ${ }^{46}$.

Oral ingestion of vinegar rapidly increases circulating acetate as observed in healthy participants that increased serum acetate levels from $120 \mu \mathrm{mol} / \mathrm{l}$ during placebo conditions up to $350 \mu \mathrm{mol} / 1$ (after $15 \mathrm{~min}$ ) and $200 \mu \mathrm{mol} / 1$ (after $30 \mathrm{~min}$ ) after vinegar (100 ml containing $0.75 \mathrm{~g}$ acetic acid) and acetic acid capsules (containing $0.75 \mathrm{~g}$ of acetic acid) intake, respectively ${ }^{47}$. Acetic acid is a bioactive component with a dominant flavor in different types of vinegars including cider, malt, plum, sherry, tomato and wine vinegar ${ }^{48}$. In addition, vinegars may contain other polyphenol residual components (i.e. gallic acid, catechin) such as in apple cider, grape, sherry and Balsamic vinegar ${ }^{48}$. Therefore, it is important to consider the vinegar type since their composition of phenolic, flavonoid and acetic acid content may differ ${ }^{49}$.

In general, various dietary products such as preservatives, acidity regulators, food substitutes, ethanol and vinegar may provide acetate orally. In particular, vinegar may provide rapid increments in plasma acetate levels due to its fast absorption in the upper digestive tract (See Vinegar administrations in humans). However, future vinegar supplementations should specify detailed composition including acetic acid percentage and polyphenols content. 


\subsection{Microbial-derived acetate production}

Microbial-derived acetate production is yielded by the fermentation of indigestible foods in particular of acetogenic fibers (e.g. galacto-oligosaccharides, inulin) ${ }^{50}$. In postprandial conditions, acetogenic fibers can be fermented and may elevate production of acetate in the proximal colon (See acetogenic fibers in human studies) ${ }^{51}$. When acetogenic fibers reach the colon, acetate is mainly generated by the microbial community via two metabolic pathways: acetogenesis and the carbon fixation pathway ${ }^{52}$. Acetogenesis is the production of acetate, mediated by homoacetogenic bacteria or acetogens (found in the digestive tract of humans and ruminants) which are capable to produce acetate from $\mathrm{H}_{2}$ and carbon dioxide $\left(\mathrm{CO}_{2}\right)$ 53. As part of acetogenesis, the carbon fixation pathway (also known as WoodLjungdahl pathway) may also produce acetate from $\mathrm{CO}_{2}$ as precursor ${ }^{52}$.

In addition, acetate may originate from microbial fermentation of residual peptides and fats ${ }^{54-56}$. For instance, HFD-fed rats (60\% fat) showed elevated colonic and whole-body acetate turnover together with a shift on the phylum level (increased Firmicutes/Bacteroidetes ratio) ${ }^{57}$. Additionally, microbiota transplant of HFD-fed to germ-free rats increased acetate turnover ${ }^{57}$. Of note, in a Western diet (low fiber intake) protein fermentation occurs mainly in the distal colon where saccharolytic substrates are depleted ${ }^{58}$ and this produces other compounds of toxic nature such as ammonia, amines, phenols and sulfides ${ }^{59}$. Branched chain and aromatic amino acids may be produced and further metabolized via cross-feeding mechanisms and alter gut integrity and impair insulin sensitivity ${ }^{60}$. In summary, gut-derived acetate production is determined by the balance between saccharolytic and proteolytic fermentation, in particular by the presence of acetogenic fibers and it is tightly regulated by the intricate interplay within the microbial community.

Although gut-derived acetate production is expected to be low during states of low presence of fibers such as fasting, some studies suggest a possible contribution of fasting-induced alterations in the gut microbiota to fasting acetate concentrations ${ }^{61}$. This was accompanied by an increase in Firmicutes/Bacteroidetes ratio and cross-feeding mechanisms as evidenced by an upregulation of pyruvate fermentation pathways to acetate and lactate by Lactobacillus reuteri and other unclassified bacteria ${ }^{61}$. In support, human fasting and caloric restriction interventions have described an increase in microbial diversity and abundance of important acetate producers, such as Akkermansia muciniphila (A. muciniphila) and Bifidobacterium ${ }^{62,63}$.

\subsection{Colonic and systemic acetate concentrations}

Acetate concentrations in the colon start with the highest levels in the caecum $(69 \mathrm{mmol} / \mathrm{l})$ and ascending colon $(63 \mathrm{mmol} / \mathrm{l})$, followed by a subsequent decrease in the transverse $(57.9 \mathrm{mmol} / \mathrm{l})$, descending $(43.5 \mathrm{mmol} / \mathrm{l})$ and sigmoid colon (50.1 mmol) (measured by $\mathrm{kg}$ of intestinal luminal contents) as observed in sudden death victims ${ }^{22}$. This progressive decline along the colon suggests that 
major acetate production and absorption occurs in the proximal colon. In general, microbially produced acetate in the proximal colon may follow a colonic-hepaticperiphery distribution starting with colonic levels in the $\mathrm{mmol} / \mathrm{l}$ range followed by a significant drop, around 10-fold in the liver and reaching the periphery in the $\mu \mathrm{mol} / 1$ range (See Table 1 . Circulating acetate in humans) ${ }^{22}$.

\begin{tabular}{|c|c|c|c|}
\hline Condition & $\begin{array}{l}\text { Site and average (SEM) } \\
\text { concentrations }\end{array}$ & Population & Study \\
\hline Fasting & $\begin{array}{l}\text { Superior mesenteric vein } 50.4 \pm \\
11.3 \mu \mathrm{mol} / 1 \\
\text { Inferior mesenteric vein } 102.7 \pm \\
27.2 \mu \mathrm{mol} / 1 \\
\text { Portal vein } 41.4 \pm 7.8 \mu \mathrm{mol} / 1 \\
\text { Hepatic vein } 23.6 \pm 4.8 \mu \mathrm{mol} / 1 \\
\text { Radial artery } 21.8 \pm 7.6 \mu \mathrm{mol} / 1\end{array}$ & $\begin{array}{l}\text { Healthy/Overweight upper abdominal } \\
\text { surgery patients (54-75 yr.) }\end{array}$ & $\begin{array}{l}\text { Neis et al. } \\
(2018)^{64}\end{array}$ \\
\hline Fasting & Peripheral vein $44 \pm 4.4 \mu \mathrm{mol} / 1$ & Healthy/Ileostomy (56-80 yr.) patients & $\begin{array}{l}\text { Scheppach } \\
\text { et al. (1991) } 65\end{array}$ \\
\hline Fasting & $\begin{array}{l}\text { Small intestine } 77.6 \pm 3.23 \\
\mathrm{mmol} / \mathrm{kg} \\
\text { Large intestine } 53.72 \pm 9.87 \\
\mathrm{mmol} / \mathrm{kg} \\
\text { Portal vein } 258 \pm 40.13 \mu \mathrm{mol} / 1 \\
\text { Hepatic vein } 115 \pm 28.20 \mu \mathrm{mol} / 1 \\
\text { Peripheral vein } 70 \pm 18.55 \\
\mu \mathrm{mol} / \mathrm{l}\end{array}$ & Sudden death victims (16-89 yr.) & $\begin{array}{l}\text { Cummings } \\
\text { et al. (1987) } 22\end{array}$ \\
\hline Fasting & $\begin{array}{l}\text { Peripheral vein } 53.8 \pm 4.44 \\
\mu \mathrm{mol} / 1 \\
\begin{array}{l}\text { Peripheral } \\
\mu \mathrm{mol} / 1\end{array}\end{array}$ & Healthy patients (19-41 yr.) & $\begin{array}{l}\text { Pomare et al. } \\
(1985)^{51}\end{array}$ \\
\hline
\end{tabular}

Interestingly, a recent study that sampled acetate simultaneously from different colonic sites (proximal, distal), inferior/superior mesenteric veins (IMV/SMV), portal/hepatic vein and radial artery in patients undergoing surgery found acetate release was the highest in the IMV $(102.7 \pm 27.2 \mu \mathrm{mol} / \mathrm{l})$ and lowest in the radial artery $(21.8 \pm 7.6 \mu \mathrm{mol} / \mathrm{l})$. They reported a correlation of arterial acetate concentrations with those in the IMV $\left(\mathrm{r}^{2}=0.65, \mathrm{p}<0.01\right)$, not with SMV, and much lower concentrations in hepatic and portal veins $(23.6 \pm 4.8 \mu \mathrm{mol} / 1$ and $41.4 \pm 7.8$ $\mu \mathrm{mol} / \mathrm{l}$, respectively). Collectively, this suggested a greater release from the distal colon ${ }^{64}$. This highlighted the distal colon as a potential location to promote acetogenic fiber fermentation and/or as the location to test the effectiveness of a fiber supplementation in future studies.

A rodent study identified increments in circulating acetate concentrations after intraperitoneal infusion and oral gavage, but not after acetate administration in drinking water ${ }^{66}$. Nevertheless, some studies that provided acetate in drinking water have reported beneficial effects ${ }^{67-69}$. However, the study ${ }^{66}$ suggests that acetate effects/kinetics and clearance may depend on administration site of exogenous acetate. However, it should be mentioned that this study did not measure acetate with the gold standard technique (electron ionization gas 
chromatography mass spectroscopy) instead it used a commercially available colorimetric kit that allowed the measurement of multiple time points (each measurement required only $\sim 1 \mu \mathrm{L}$ of plasma) ${ }^{66}$. In summary, acetate production and colonic acetate release may vary along the colon. Thus, the site of fermentation and acetate production may be an important determinant of circulating concentrations.

\subsection{Prebiotics and bacterial acetate producers}

\subsubsection{Prebiotic in vitro studies}

First, using an in vitro system mimicking colonic digestion ${ }^{70}$ researchers reported that fermentation of starch entrapped microspheres, fructooligosaccharides (FOS) and Psyllium led to substantial in vitro acetate production

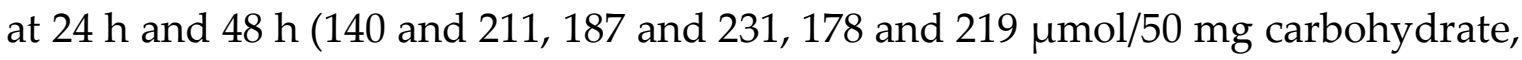
respectively). However, corn bran arabinoxylans in the same system showed a higher acetate production after 24 and 48 hours $(222,284 \mu \mathrm{mol} / 50 \mathrm{mg}$ carbohydrate, respectively) ${ }^{71}$. Furthermore, using the proximal large intestine in vitro TIM-2 model (developed by TNO, Zeist, The Netherlands), the addition of the acetogenic fiber galacto-oligosaccharides (GOS) for $72 \mathrm{~h}$ increased total SCFA (231 vs 144 control mmol/l, respectively) and acetate production ( $+49 \%)$ to approximately 500 $\mu \mathrm{mol} / 50 \mathrm{mg}$ of carbohydrates ${ }^{72}$. Another in vitro study, comparing FOS vs. inulin mixtures with various degrees of polymerization (DP) reported that a mixture of inulin (90-94\% DP>10 and 6-10\% DP=1-2) showed the greatest acetate levels at $24 \mathrm{~h}$ $(25.1 \mathrm{mmol} / \mathrm{l})$ and a mixture of Oligofructose $(>93.2 \% \mathrm{DP}<10$ and $<6.8 \% \mathrm{DP}=1)$ at 4 $\mathrm{h}$ increased the levels $(39.7 \mathrm{mmol} / \mathrm{l})$, however not reaching significance ${ }^{73}$. Similarly, a comparison of inulin (DP 3-60) versus Oligofructose (DP 2-20) with various DP using the Simulator of Human Intestinal Microbial Ecosystem (SHIME) in vitro model, reported that inulin with longer chain lengths (i.e. Chicory) showed a slower breakdown, more beneficial changes in microbial community (both proximal and distal), higher acetate production and higher bifidogenic effect in the distal colon than oligofructose ${ }^{74}$. In addition, the glycosidic bond configuration of dietary fibers may affect acetate production ${ }^{75}$. For instance, different glycosidic bonds in the $\beta$ orientation led to differences in acetate production (diglucose $\beta$ 1-6 vs diglucose $\beta 1-4,3.7 \pm 0.3$ vs $1.9 \pm 0.2 \mathrm{mmol} / \mathrm{g}$ carbohydrate/day, $\mathrm{P}=0.001$ ) but it did not differ between fibers with various glycosidic bond configurations ( $\alpha$ and $\beta$ ) 76.

With respect to timing, a recent in vitro study concluded that fermentation was delayed more when a mixture (arabinoxylan, chondroitin sulfate, galatomannan, polygalactunoric acid and xyloglucan) rather than when the individual fibers were provided. Of note, they suggested that mixtures of fibers may promote the late fermentation of soluble fibers in the distal colon and prevent deleterious effects of proteolytic activity ${ }^{77}$. Collectively, these studies have demonstrated, by the use of in vitro fermentation models, the potential of prebiotic fibers as well as the importance of fiber characteristics (i.e. DP, glycosidic bond 
configuration, orientation, chain length) to produce acetate. However, future studies are needed to investigate whether these results on delayed colonic acetate production can be replicated in humans and to what extent acetate is further absorbed and metabolized in humans.

\subsubsection{Bacterial acetate producers}

Acetate production is widely distributed within the bacterial community, as many bacterial species are acetate producers ${ }^{78}$. However, some species seem to be major acetate producers. For instance, humanized gnotobiotic mice that were cocolonized with Bacteroides thetaiotaomicron/methanobrevibacter smithii reported higher serum acetate levels as compared to Bacteroides thetaiotaomicron alone ${ }^{79}$. In overweight/obese individuals, a positive association between fecal $A$. muciniphila and serum acetate $\left(\mathrm{r}^{2}=0.36 \mathrm{p}=0.01\right)$ at baseline was described, however, 6-week caloric restriction resulted in decreased serum acetate levels and disappearance of the associations which indicated sensitivity to diet changes ${ }^{63}$. Of importance, differences in abundances of species along the colon may contribute to differences in acetate production between the proximal and distal colon ${ }^{80,81}$. According to the SHIME in vitro model, A. muciniphila colonization preferred high $\mathrm{pH}$ (6.6-6.9) and high concentration of mucin, which are more abundantly present in the distal compared to the proximal colon ${ }^{82}$. In support, murine studies have reported higher abundance of $A$. muciniphila in the colon as compared to the ileum ${ }^{83,84}$. Moreover, in vitro models that mimic the human colon reported highest concentration of $A$. muciniphila in the transverse compartment, followed by the descending compartment and no detection in the ascending colon ${ }^{85}$. Further discussion on important acetate microbial producers is continued under therapeutic approaches (See Probiotics, body weight control and insulin sensitivity).

\section{Acetate in control of body weight}

Acetate administration or microbially-derived acetate may have an impact on body weight control through effects on energy intake as well as energy expenditure. In a 12-week intervention in HFD-fed mice, Den Besten et al. ${ }^{86}$ found that oral sodium acetate supplementation incorporated into the diet (at 5\%, wt./wt. proportion) resulted in a suppression of HFD-induced weight gain $(\sim 30 \%)$ compared to control mice fed a HFD ${ }^{86}$. Similarly, Lu et al. ${ }^{87}$ showed that 16 -weeks of oral sodium acetate supplementation (5\%, wt./wt.) suppressed HFD-induced weight gain by $72 \%(\mathrm{p}<0.05)$ in comparison to control mice fed a HFD. In addition, a 6-week intragastric administration of acetic acid (50, $250 \mathrm{mmol} / \mathrm{l})$ to HFD-fed mice reduced weight gain $(7,8 \%$, respectively) and body fat accumulation in comparison to HFD alone ${ }^{88}$.

In contrast, Perry et al. ${ }^{57}$ described that both continuous intragastric acetate infusions (10 days, at a rate of $20 \mu \mathrm{mol} \mathrm{kg} \mathrm{min}^{-1}$ ) and a HFD ( $3 \mathrm{~d}$ and 4 weeks) in rats led to similar increased systemic ghrelin, gastrin concentrations and glucose 
stimulated insulin secretion (GSIS) that collectively promoted hyperphagia and energy retention that ultimately caused weight gain. Importantly, this elegant rodent study provided valuable mechanistic insight in acetate role in metabolic health although conflicting with other important rodent studies ${ }^{86-89}$. The differences might be related to the species and phenotype of animals used (i.e. Sprague-Dawley rats versus $\mathrm{C} 57 \mathrm{BI} / 6 \mathrm{~J}$ mice) or administration site (intragastric versus oral or colonic administration). This further highlights the need to investigate metabolic effects of an increased whole-body acetate turnover in humans. Of note, in contrast to the outcomes of Perry et al, a recent human study conducted by the same group failed to corroborate these findings, reporting a higher acetate turnover in lean $(\sim 30 \%)$ versus obese individuals and no effect of increased circulating acetate levels (intravenous infusion) on ghrelin and GSIS in obese individuals ${ }^{90}$. These important data showed that there are important differences across species and between modes of administrations.

In humans, long-term oral acetate supplementation or intravenous/gastric/colonic infusion studies with weight loss and energy expenditure as the primary outcome are limited (See Acetate effects on energy expenditure). Cross-sectional/cohort analyses have shown inconsistent results with obesity and adiposity. For instance, one study showed that fasting acetate correlated negatively with visceral adipose tissue mass in obese women ${ }^{91}$. In contrast, in obese men and women a positive association $\left(\mathrm{r}^{2}=0.11, \mathrm{P}=0.004\right)$ of fasting plasma acetate $(0-2 \mu \mathrm{mol} / 1$ range $)$ with degree of adiposity at baseline including total body, visceral and subcutaneous fat (magnetic resonance imaging) was observed independent of age, sex and ethnicity ${ }^{92}$.

In addition, baseline fasting plasma acetate positively predicted changes in adiposity (delta BMI per year) after a $2.2 \pm$ 1.7-year follow-up time independent of baseline BMI, age, sex and ethnicity. Additionally, fasting plasma acetate levels correlated positively with de novo fasting hepatic lipogenesis (measured by hepatic palmitate incorporation) ${ }^{92}$. As mentioned above, long-term oral acetate supplementation studies in humans are lacking and data on the role of acetate in body weight management are mainly based on animal data and human crosssectional data. The above discrepancies highlight that acetate effects on body weight control may depend on mode of administration, metabolic phenotype and species-specific differences in acetate metabolism. In the following paragraphs, we discuss the putative mechanisms of how acetate might influence body weight control via central nervous system mechanisms and gut-derived hormones as well as via effects on energy expenditure. 


\subsection{Acetate and central effects on appetite regulation}

Appetite regulation is coordinated by nutrients and microbial metabolites through the central nervous system circuitry and circulating hormones from peripheral tissues ${ }^{93}$. Of note, acetate has been reported to cross the blood-brain barrier in both mice ${ }^{89}$ and humans ${ }^{94}$. Additionally, acetate has been detected in the cerebrospinal fluid, thus suggesting a central homeostatic mechanistic role ${ }^{95}$. In support, Frost et al. ${ }^{89}$ described an association of elevated colonic acetate and a direct role of acetate on appetite regulation in HFD-fed mice supplemented for 8 weeks with acetogenic oligofructose-enriched inulin. Acetate accumulation in the hypothalamus, was shown to affect appetite regulation through the glutamateglutamine transcellular cycle which resulted in increments in lactate and gamma aminobutyric acid (GABA) production, after both intraperitoneal acetate injections and after colonic fermentation of ${ }^{13} \mathrm{C}$-labelled carbohydrate ${ }^{89}$. Interestingly, mice showed a peak in serum acetate levels $(350 \mu \mathrm{mol} / \mathrm{l})$ at $10 \mathrm{~min}$ after an intraperitoneal acetate injection $\left(500 \mathrm{mg} / \mathrm{kg}^{-1}\right)$, that was associated with changes in the expression of neuropeptides (AMPK and acetyl CoA carboxylase (ACC)) that regulate appetite suppression ${ }^{89}$. Hypothalamic acetate administration showed inactivation of AMPK and activation of ACC (via decreased phosphorylation), suggesting that acute administration of acetate may increase hypothalamic ACC activity. ACC activation may increase malonyl-CoA, which may lead to a reduction in food intake through the expression of orexigenic and anorexigenic neuropeptides in the hypothalamus via two mechanisms: (i) via the interaction of malonyl-CoA with signaling proteins or (ii) via inhibition of carnitine/palmitoylCoA transferase that prevents the entry of the long-chain fatty acids to the mitochondrion ${ }^{96,97}$. Of note, these data are of associational nature.

Therefore, to assess a causal role of these pathways and to further study the role of acetate on appetite and satiety regulation animal models (i.e. MC4R-/- mice) might be used.

Interestingly, acetate signaling in the brain may follow a hepatic-portalvagal route. However, vagal activation and effects on body weight control may depend on site of administration and use of different animal models ${ }^{98}$. For instance, Perry et al. ${ }^{57}$ reported that both a HFD and continuous intragastric infusions of acetate increased central vagal activation, including a vagal-induced release of ghrelin from the stomach that led to a metabolic syndrome phenotype in rats as explained above. In mice, an intraperitoneal acetate injection (6 mmol/ $\mathrm{kg}$ ) significantly reduced food intake ( 0.5 and 1 hour after administration) in a vagal dependent manner since vagotomy attenuated the effect ${ }^{99}$. Although these studies may imply that vagal activation in response to acetate are contradictory, it is important to note that the site of administration and use of different animal models may explain the difference in vagal activation. In addition, energy intake may also regulate vagal activation by gut hormones (GLP-1, glucagon like peptide 1 and 
peptide $Y Y, P Y Y)$, as vagotomized humans showed impaired effects of exogenous GLP-1 on food intake ${ }^{100}$.

In summary, acetate may regulate appetite possibly through central hypothalamic mechanisms and satiety through acetate-induced or gut hormoneinduced vagal activation. However, due to the lack of human evidence and the inconsistency in animal data, further research should elucidate the exact underlying mechanism.

\subsection{Gut-derived satiety hormones}

As indicated above, gut-derived satiety hormones can be secreted from enteroendocrine cells located in the gut ${ }^{101}$. Enteroendocrine cells, in particular the L-cells, secrete GLP-1 and PYY hormones that seem to play an important role in gut health and in the connection of the gut-brain axis ${ }^{102}$. Notably, both acetate receptors GPR43 ${ }^{23}$ and GPR41 ${ }^{24}$ are expressed at mRNA and protein level in enteroendocrine cells in the human colonic mucosa, thus potentially indicating an acetate-mediated effect in their secretion ${ }^{103}$. In support, in vitro culturing of enteroendocrine cells (SCT-1 cell line) with acetate ( 3 and $30 \mathrm{mmol} / \mathrm{l})$ for $24 \mathrm{~h}$ showed increased expression of proglucagon (GLP-1 precursor) in a dose-dependent manner ${ }^{104}$. In a mice study, inulin supplementation protected from HFD-induced obesity, which was dependent on an increased PYY secretion in a GPR43 dependent manner ${ }^{105}$. In addition, dietary fiber supplementation in rats with resistant starch increased plasma levels of GLP-1 and PYY in the short term (24-hour period) ${ }^{104}$. Similarly, rats supplemented with resistant starch (30-day period) increased plasma levels of GLP-1 and PYY and increased caecal acetate ${ }^{106}$. Using inulin type fructans, Cani et al. ${ }^{107}$ demonstrated that a 3-week oral supplementation in rats increased GLP-1 and decreased ghrelin plasma levels, possibly via colonic SCFA production, in particular of acetate. In addition, feeding of dogs with a mixture of high fermentable fibers for 14 days increased plasma GLP-1 concentration 15 min after an oral glucose load, however, systemic acetate was not measured ${ }^{108}$.

Importantly, GPR41 has been reported in other enteroendocrine cell types (secretin and neurotensins types) in the duodenum and proximal colon of mice, respectively ${ }^{25}$. However, whether acetate can induce a GPR41-dependent secretion of these appetite-suppressing hormones needs further investigation. In humans, production of gut-derived satiety hormones in response to prebiotic supplementation is scarce and inconclusive. For instance, Rahat-Rozenbloom et al. ${ }^{109}$ reported an increase in colonic acetate but no increase in serum levels of satiety hormones (GLP1, PYY) in overweight individuals after inulin ingestion (single dose $24 \mathrm{~g}$ ) in comparison to glucose as control. In contrast, oligofructose supplementation (21 g/day) for 12 weeks in overweight/obese individuals increased the area under the curve (AUC) of PYY, and decreased AUC of ghrelin secretion.

This coincided with a reduction in self-reported caloric intake and was associated with a significant weight loss $(1.03 \pm 0.43 \mathrm{~kg})$ as compared to maltodextrin ${ }^{110}$. In addition, oligofructose $(35 \mathrm{~g} / \mathrm{d})$ in lean individuals, increased 
postprandial PYY concentrations 111. Importantly, these human prebiotic interventions did not measure fecal/systemic acetate. Another approach to investigate secretion of gut-derived hormones is through colonic acetate infusions.

Interestingly, infusions in humans in different sites may elicit differential effects on gut-derived satiety hormones. For example, rectal $(60 \mathrm{mmol} / \mathrm{l})$ sodium acetate administrations increased PYY and GLP-1 significantly as compared to intravenous infusions (acetate $20 \mathrm{mmol} / 1$ and saline) ${ }^{112}$. Similarly, distal (not proximal) colonic infusions of sodium acetate $(180 \mathrm{mmol} / \mathrm{l})$ increased PYY in overweight/obese men and only $180 \mathrm{mmol} / 1$ (not $100 \mathrm{mmol} / \mathrm{l}$ ), elicited these effects 39. Moreover, rectal/intravenous infusions showed the potential to induce gutderived hormone secretion (GLP-1 and PYY) in a metabolically disturbed phenotype ${ }^{113}$. Together, these distal colonic infusions studies suggest the relevance of the site of administration or fermentation (distal versus proximal colon) to modulate gut hormone secretion. Of note, a higher density of PYY producing cells in the distal colon in rodent studies ${ }^{114,115}$ may explain the differential effect on hormonal secretion dependent on site of fermentation.

Acetate may also increase the secretion of leptin from the adipose tissue. For instance, in a rodent study, acetate $(860 \mu \mathrm{mol} / \mathrm{l})$ increased transcription of the leptin gene, and propionate $(78 \mu \mathrm{mol} / \mathrm{l})$ showed even stronger effects ${ }^{116}$. In bovine adipocytes, acetate $(1 \mathrm{mmol} / \mathrm{l})$ increased leptin expression by $\sim 60 \%$, which was inhibited by pertussis toxin, indicating GPR dependence ${ }^{117}$. Further studies have to show the relevance of this mechanism under in vivo conditions. In summary, animal and in vitro data studies have shown the potential of acetate to increase gut hormone secretion, however, dietary fiber supplementation in humans have shown inconsistent results. Future human trials may aim to target distal colonic fermentation since distal colonic acetate infusions have shown pronounced increments in gut satiety hormones.

\subsection{Acetate effects on energy expenditure}

A few studies have investigated the direct effects of acetate on energy expenditure as a primary outcome. Of note, acetate has been related to increments in energy expenditure through various mechanisms in peripheral tissues ${ }^{41}$ (See Acetate inter-organ crosstalk and insulin sensitivity in peripheral tissues). Here we discuss the effects of acetate infusions in humans and also in the form of vinegar through oral administrations in the context of body weight control.

\subsubsection{Acetate infusions in humans and energy expenditure}

In humans, a lipid lowering effect of acetate after rectal ${ }^{118}$ and intragastric 119 infusions in healthy subjects almost three decades ago paved the way for future human acetate infusions (See Table 2). Recently, acute colonic infusions showed increments in fasting fat oxidation and energy expenditure in humans ${ }^{39,120}$. First, distal colonic infusions in normoglycemic overweight/obese individuals increased fasting fat oxidation $\left(1.78 \pm 0.28\right.$ vs $-0.78 \pm 0.89 \mathrm{~g}$ fat $\left.2 \mathrm{~h}^{-1}, \mathrm{P}=0.015\right)$, however no 
effects on energy expenditure ${ }^{39}$. Another study in the same phenotype, using SCFA mixtures (rich in acetate) reported positive associations of fasting acetate with fasting fat oxidation $(\mathrm{r}=0.328 \mathrm{P}=0.0228)$ and with resting energy expenditure $(\mathrm{r}=0.349 \mathrm{P}=0.0149){ }^{120}$. In contrast, a study in healthy and T2DM subjects, acetate intravenous infusions $(2.5 \mathrm{mmol}$ per $\min$ for $1 \mathrm{~h}$ ) did not increase energy expenditure, which was partly explained by the fact that acetate might replace long chain fatty acids as preferred oxidation fuel ${ }^{121}$. Collectively, these studies suggested an acetate-mediated beneficial role in substrate utilization and energy expenditure in humans, however, results are inconclusive and further research is needed. In addition, the beneficial effects after distal colonic (not proximal) acetate infusions ${ }^{39}$, together with the higher acetate release in distal colon ${ }^{64}$ strengthened the notion that targeting distal colonic site might increase the metabolic health effects. 


\begin{tabular}{|c|c|c|c|c|c|}
\hline Dose & $\begin{array}{l}\text { Primary } \\
\text { outcome }\end{array}$ & Subjects & $\begin{array}{l}\text { Administratio } \\
\text { n site }\end{array}$ & $\begin{array}{l}\text { Effects on } \\
\text { lipid/glucose } \\
\text { metabolism }\end{array}$ & Study \\
\hline $\begin{array}{l}\text { SCFA } \text { infusions } \\
\text { mixtures rich in } \\
\text { acetate }(24 \mathrm{mmol} / 1 \\
\text { acetate, } 8 \mathrm{mmol} / 1 \\
\text { propionate and } 8 \\
\text { mmol/1 butyrate) } \\
\text { and propionate (18 } \\
\text { mmol/l acetate, } 14 \\
\text { mmol/l propionate } \\
\text { and } 8 \text { mmol/l } \\
\text { butyrate) }\end{array}$ & $\begin{array}{l}\text { Fat oxidation and } \\
\text { Energy } \\
\text { expenditure }\end{array}$ & $\begin{array}{l}\text { Overweight/ } \\
\text { obese men } \\
(n=12)\end{array}$ & $\begin{array}{ll}\text { / } & \text { Colonic } \\
\text { n infusions }\end{array}$ & $\begin{array}{l}\text { Attenuation of whole- } \\
\text { body lipolysis } \\
\uparrow \quad \text { Fat oxidative } \\
\text { capacity } \\
\text { Fat oxidation and } \\
\text { energy expenditure } \\
\text { related to increments } \\
\text { in fasting acetate } \\
\uparrow \text { Fasting and } \\
\text { postprandial PYY } \\
\text { No effects on insulin } \\
\text { and glucose }\end{array}$ & $\begin{array}{l}\text { Canfora et } \\
\text { al. } 2017^{120}\end{array}$ \\
\hline $\begin{array}{l}180 \mathrm{mmol} / \mathrm{l} \\
\text { sodium acetate }\end{array}$ & $\begin{array}{l}\text { Fat oxidation and } \\
\text { energy } \\
\text { expenditure }\end{array}$ & $\begin{array}{l}\text { Overweight/ } \\
\text { obese men } \\
(n=6)\end{array}$ & $\begin{array}{l}\text { Proximal } \\
\text { and Distal } \\
\text { colonic }\end{array}$ & $\begin{array}{l}\uparrow \text { Fasting fat oxidation } \\
\uparrow \text { Postprandial glucose } \\
\text { and insulin } \\
\text { Tendency to decrease } \\
\text { TNF- } \alpha \\
\text { Fasting peptide YY }\end{array}$ & $\begin{array}{l}\text { Van der } \\
\text { Beek et al. } \\
(2016)^{39}\end{array}$ \\
\hline $\begin{array}{l}140 \mathrm{mmol} / \mathrm{l} \text { in } 90 \\
\text { min sodium acetate }\end{array}$ & $\begin{array}{l}\text { Peripheral } \\
\text { uptake }\end{array}$ & $\begin{array}{l}\text { Overweight } \\
\text { normoglyce } \\
\text { mic and } \\
\text { hyperglyce } \\
\text { mic subjects } \\
\text { (n=9 vs } 9 \text { ) }\end{array}$ & IV & $\begin{array}{l}\text { No difference in } \\
\text { acetate clearance } \\
\text { between individuals } \\
\text { with normal (NI) and } \\
\text { high (HI) insulin } \\
\text { levels. } \\
\uparrow \text { FFA rebound in NI } \\
\text { than HI. }\end{array}$ & $\begin{array}{l}\text { Fernandes } \\
\text { et al. (2012) } \\
113\end{array}$ \\
\hline $\begin{array}{l}60 \mathrm{mmol} / \mathrm{l} \text { (rectal), } \\
20 \quad \mathrm{mmol} / 1 \\
\text { (intravenous) } \\
\text { Sodium acetate }\end{array}$ & $\begin{array}{l}\text { Gut-derived } \\
\text { hormone } \\
\text { secretion }\end{array}$ & $\begin{array}{l}\text { Hyperinsuli } \\
\text { naemic } \\
\text { females } \\
(\mathrm{n}=6)\end{array}$ & $\begin{array}{l}\text { Rectally and } \\
\text { IV }\end{array}$ & $\begin{array}{l}\uparrow \text { in PYY/GLP-1 after } \\
\text { rectal infusions } \\
\text { and decrease in TNF }\end{array}$ & $\begin{array}{l}\text { Freeland et } \\
\text { al. (2010) }\end{array}$ \\
\hline $12 \mathrm{mmol} / \mathrm{l}$ per hour & $\begin{array}{l}\text { Hepatic glucose } \\
\text { production }\end{array}$ & $\begin{array}{l}\text { Healthy } \\
\text { subjects } \\
(n=6)\end{array}$ & IG & $\begin{array}{l}\downarrow \text { Circulating FFA } \\
\text { No effect on hepatic } \\
\text { glucose production }\end{array}$ & $\begin{array}{l}\text { Laurent et } \\
\text { al. (1995) } \\
119\end{array}$ \\
\hline $\begin{array}{l}800 \quad \mathrm{ml} \text { rectal } \\
\text { infusions with } 180 \\
\mathrm{mmol} / \mathrm{l}\end{array}$ & $\begin{array}{l}\text { Glucose } \\
\text { homeostasis }\end{array}$ & $\begin{array}{l}\text { Healthy } \\
\text { subjects } \\
(\mathrm{n}=6)\end{array}$ & $\begin{array}{l}\text { Rectal } \\
\text { infusion }\end{array}$ & $\begin{array}{l}\text { No effects on insulin } \\
\text { and glucose } \\
\downarrow \text { Circulating FFA }\end{array}$ & $\begin{array}{l}\text { Wolever et } \\
\text { al. (1989) }{ }^{118}\end{array}$ \\
\hline
\end{tabular}

Abbreviations: SCFA, short chain fatty acids; PYY, peptide YY; GLP-1, glucagon like-peptide 1; TNF- $\alpha$, Tumor necrosis factor; FFA, free fatty acid; IV, Intravenous; IG, Intragastric.

\subsubsection{Vinegar administrations in humans}

Studies related to vinegar effects on body weight and energy expenditure in humans are limited. Nevertheless, a few studies have reported effects on body weight. For instance, a study in individuals with obesity, a 12-week vinegar intervention significantly lowered body weight with low (0.75 gr) and high (1.5 gr) acetate doses versus placebo ( $0 \mathrm{gr})$, in a dose-dependent manner ${ }^{122}$. 
In addition, a crossover study in overweight-obese subjects, the consumption of Kimchi (fermented Korean dish, unclear \% of acetic acid) vs unfermented dish reduced body fat $(\sim 1 \%)$, body weight $(\sim 1.5 \mathrm{~kg})$ and BMI $(0.6$ $\mathrm{kg} / \mathrm{m}^{2}$ ) after 2 weeks ${ }^{123}$. In a similar crossover study, in overweight women, fermented Kimchi (unclear \% of acetic acid) decreased Firmicutes/Bacteroidetes ratio 124 which has been associated with weight loss ${ }^{125}$. In HFD-fed mice, supplementation of synthetic acetic acid (4\%) and high dose of Nipa vinegar (unclear \% of acetic acid) reduced lipid deposition, inflammation and improved serum lipid profiles in comparison to control. Both vinegars decreased Firmicutes/Bacteroidetes ratio and increased the relative abundances of various bacterial species including A. muciniphila and Lactobacillus among other potential acetate producers ${ }^{126}$. With respect to differences in acetate infusions (sodium acetate) versus vinegar (acetic acid) administrations, both the route and absorption may differ. Saunders et al. ${ }^{127}$, reported that oral acetic acid administration was more rapidly absorbed in the stomach compared with sodium acetate administrations, possibly through a $\mathrm{pH}$-dependent mechanism, as acetic acid (unionized acetate) absorption increased when gastric $\mathrm{pH}$ decreased.

Although, in previous human and rodent studies the effects were attributed to acetic acid, either its content (\%) or the presence of other bioactive components is unclear. In addition, fermented dishes (e.g. Kimchi) may have other volatile and non-volatile compounds and overall composition can vary depending on fermentation time and storage room temperature ${ }^{128}$. In general, acetate may modulate body weight control through different mechanisms that can affect central appetite regulation, gut-satiety hormones as well as improvements in lipid metabolism and energy expenditure. Of note, human evidence is accumulating that acetate may prevent body weight gain and adiposity through increments in energy expenditure as observed in following acute colonic distal infusion studies. Importantly, there is a lack of longer-term human studies that investigate acetate effects on energy expenditure as primary outcome. In the next section, we discuss acetate effects in the peripheral tissues that collectively may improve insulin sensitivity (See Table 3). 


\section{Acetate and the inter-organ crosstalk and insulin sensitivity in peripheral tissues}

Adipose tissue is the main organ for triacylglycerol storage in the human body and an active endocrine regulator of energy homeostasis, therefore metabolic derangements in adipose tissue function contribute to pathophysiology and dysregulation of glucose homeostasis and whole-body insulin sensitivity ${ }^{129}$.

\subsection{Acetate and vinegar studies and insulin sensitivity}

Of note, human acute acetate infusions have shown inhibitory roles in whole-body lipolysis, increase in gut-hormone release and increase in fat oxidation and energy expenditure among other effects (See Table 2). Collectively, these effects may improve adipose tissue lipid buffering capacity, satiety regulation, oxidative capacity and in turn improve whole-body insulin sensitivity and peripheral tissue functioning. In addition, vinegar administrations have reported improvements in glucose homeostasis and insulinemic profiles (See Table 3). Moreover, we discuss vinegar effects on glucose homeostasis and insulinemic profiles with potential T2DM treatment applications ${ }^{46}$.

As mentioned above, oral vinegar (4-8\% acetic acid) administrations may rapidly increase circulating acetate and its co-ingestion with carbohydrates (50-75 gr) seems more effective for glucose lowering and insulinemic responses. In contrast to colonic sodium acetate infusions, oral vinegar administrations have shown improvements in glucose homeostasis and insulin profiles in healthy subjects ${ }^{46,130,131}$. For instance, supplementation of acetic acid (unspecified vinegar) in healthy subjects together with a test meal resulted in reduced postprandial glucose concentration $(\sim 35 \%$, during $30-70 \mathrm{~min})$, putatively through a delayed gastric emptying ${ }^{130}$. Similarly, white vinegar ( $6 \%$ acetic acid) administrations (18, 23 and $28 \mathrm{mmol} / \mathrm{l})$ in combination with white wheat bread (50 gr) in healthy subjects lowered glycemic (highest dose at 30-45 min) and insulinemic (highest dose at 15-30 min) postprandial responses ${ }^{132}$. In addition, acetic acid lowered the glycemic index (GI) and increased the satiety score postprandially at 30, 90 and 120 min using a subjective rating scale ${ }^{132}$. Another study in healthy subjects, reported that a vinaigrette ( $28 \mathrm{~g}$ white vinegar, $6 \%$ acetic acid) on a potato meal reduced GI and insulinemic index (43 and 31\%, respectively) ${ }^{133}$.

Furthermore, vinegar administration studies in individuals with metabolic alterations (i.e. impaired glucose tolerance IGT, T2DM) have been performed ${ }^{46}$. For instance, a study in individuals with IGT showed that wine vinegar (6\% acetic acid) administration decreased arterial plasma insulin (by 33\%) and increased muscle glucose uptake (by $35 \%$ ) after a meal test as compared to placebo (50 $\mathrm{ml}$ water) ${ }^{134}$. Additionally, in T2DM individuals, oral wine vinegar administration (1.2 g acetic acid) decreased iAUC $120 \mathrm{~min}$ of glucose (41\%) only after a high GI meal test (mashed potatoes and low-fat milk) but not after a low GI meal test ${ }^{135}$. 
However, these reported beneficial effects of vinegar on glucose homeostasis in metabolically compromised individuals have not been confirmed in all studies. A study in T2DM individuals using white vinegar (1 g acetic acid) did not show any effect on postprandial glucose levels after an oral glucose load (75 g) ${ }^{136}$. In summary, acetate infusions and vinegar administrations have reported beneficial effects on glucose homeostasis and potentially on insulin sensitivity. However, inconsistencies exist and differences between phenotypes require further research.

Table 3. Vinegar administrations in humans.

\begin{tabular}{|c|c|c|c|c|}
\hline $\begin{array}{l}\text { Dose, } \quad \text { vinegar } \\
\text { type }\end{array}$ & $\begin{array}{l}\text { Primary } \\
\text { outcome }\end{array}$ & Subjects & Effects & Study \\
\hline $\begin{array}{l}\text { Unspecified } \\
\text { vinegar }\end{array}$ & G\&IR & $\begin{array}{l}\text { Healthy }(22-51 \text { yr. }), 7 \\
\text { females } \\
(\mathrm{n}=10)\end{array}$ & $\begin{array}{ll}\downarrow & 35 \% \\
\text { postprandial } & \\
\text { glucose } & \\
\text { Delayed } & \\
\text { emptying } & \end{array}$ & Björck et al. 2005130. \\
\hline $\begin{array}{l}\text { White vinegar } 6 \% \\
\text { acetic acid }\end{array}$ & G\&IR & $\begin{array}{l}\text { Healthy }(19-27 \text { yr. }), 10 \\
\text { females } \\
(n=12)\end{array}$ & $\begin{array}{l}\downarrow \quad \text { Glucose and } \\
\text { Insulin }\end{array}$ & $\begin{array}{l}\text { Dimitriadis et al. } \\
(2015){ }^{132 .}\end{array}$ \\
\hline $\begin{array}{l}\text { White vinegar } 6 \% \\
\text { acetic acid }\end{array}$ & G\&IR & $\begin{array}{l}\text { Healthy }(19-32 \text { yr. }), 10 \\
\text { females } \\
(n=13)\end{array}$ & $\begin{array}{l}\downarrow \text { Glycemic index } \\
\text { Insulinemic index } \\
\text { (43 and } 31 \% \text {, } \\
\text { respectively) }\end{array}$ & $\begin{array}{l}\text { Diakoumoupolou et } \\
\text { al. (2010) } 133 .\end{array}$ \\
\hline $\begin{array}{l}\text { Wine vinegar } 6 \% \\
\text { acetic acid }\end{array}$ & $\begin{array}{l}\text { Muscle glucose } \\
\text { metabolism } \\
\text { Circulating } \\
\text { lipids } \\
\text { endothelial } \\
\text { function }\end{array}$ & $\begin{array}{l}\text { Individuals with } \\
\text { impaired glucose } \\
\text { tolerance (26-66 yr.), } 4 \\
\text { females } \\
(\mathrm{n}=8)\end{array}$ & $\begin{array}{l}\uparrow \quad \text { Muscle blood } \\
\text { flow } 33 \% \\
\uparrow \text { Muscle glucose } \\
\text { uptake } 35 \%\end{array}$ & $\begin{array}{l}\text { van Loon et al. (2012) } \\
134 .\end{array}$ \\
\hline $\begin{array}{l}\text { Wine vinegar } 1.2 \\
\mathrm{~g} \text { acetic acid vs } \\
\text { placebo }\end{array}$ & G\&IR & $\begin{array}{l}\text { TD2M individuals } \\
\text { ( } \mathrm{n}=8 \text { vs } 8)(40-78 \text { yr.), } 4 \\
\text { females }\end{array}$ & $\begin{array}{l}\downarrow \text { iAUC } 120 \\
\text { Glucose } 41 \%\end{array}$ & $\begin{array}{l}\text { Crovetti et al. (1995) } \\
135 .\end{array}$ \\
\hline $\begin{array}{l}\text { White vinegar } \\
1 \text { g acetic acid }\end{array}$ & G\&IR & $\begin{array}{l}\text { TD2M male } \\
\text { individuals } \\
(\mathrm{n}=12)(63-67 \mathrm{yr} .)\end{array}$ & No effect & $\begin{array}{l}\text { Haldar et al. (2016) } \\
136 .\end{array}$ \\
\hline
\end{tabular}




\subsection{Adipose tissue metabolism}

\subsubsection{Lipolysis}

Acetate administration has been shown to affect whole-body as well as intracellular lipolysis in adipocytes in in vitro, and in vivo animal and human studies. Ge et al. ${ }^{137}$ reported that acetate-mediated activation of GPR43 in 3T3-L1 adipocytes was accompanied by an inhibition of the lipolytic response within a physiological range of 100-300 $\mu \mathrm{mol} / 1{ }^{137}$. Moreover, a supraphysiological concentration of sodium acetate $(4 \mathrm{mmol} / \mathrm{l})$ induced an antilipolytic effect in murine 3T3-L1 adipocytes, via a decrease in phosphorylation of the cytosolic lipase HSL (hormone sensitive lipase) at serine residue 563 (equivalent to Ser 552 in humans) 138. Similarly, Heimann et al. ${ }^{139}$ described an antilipolytic effect of acetate, possibly mediated by a decrease in phosphorylation of HSL (at Ser 563) in rat and human primary (at Ser 552) adipocytes with a supraphysiological (10 mmol/l) concentration of acetate ${ }^{139}$. Recently, an in vitro study using differentiated human multipotent adipose-derived stem cells (hMADS) showed that acetate ( $1 \mu \mathrm{mol} / \mathrm{l}-1$ $\mathrm{mmol} / \mathrm{l}$ ) decreased basal and isoprenaline-stimulated lipolysis via attenuation of HSL Ser 650 phosphorylation (equivalent to Ser 660 in rats) in a GPR-dependent manner ${ }^{140}$.

In mice, an antilipolytic effect of acetate (30\% reduction of plasma free fatty acids) was observed after intraperitoneal infusions of sodium acetate (500 mg/kg), which coincided with a rise in plasma acetate after $15 \mathrm{~min}$ of infusion (circulating levels reached a range of 0.2-1.0 mmol/l) ${ }^{137}$. In HFD-fed mice, using a nanoparticle delivery system, acetate decreased lipolysis and circulating FFA after intraperitoneal injection ${ }^{141}$. In addition, adipose tissue mRNA expression of adipose triglyceride lipase (ATGL) was reduced.

In the human in vivo situation, a handful of studies demonstrated effects on lipolysis on whole-body level. First, Crouse et al. ${ }^{142}$ showed that orally administered sodium acetate (given in two doses $143 \mathrm{mg} / \mathrm{kg}$ initially and $71 \mathrm{mg} / \mathrm{kg}$ 30 min later) increased plasma acetate by 3 to 4 -fold and decreased FFA plasma concentrations by $25 \%$ in healthy humans within 20 min after ingestion. Second, in healthy young subjects, an intragastric infusion of sodium acetate (12 mmol/l) significantly decreased the total AUC of circulating FFA in comparison to saline infusions during five hours after infusion ${ }^{119}$. Third, rectal infusion of high dose sodium acetate $(180 \mathrm{mmol} / \mathrm{l})$ decreased serum FFA in comparison to saline infusions in healthy subjects during 2 hours after infusion ${ }^{118}$. Fourth, one-hour intravenous infusion of sodium acetate in healthy individuals increased plasma acetate from 0.19 to $0.99 \mathrm{mmol} / \mathrm{l}$ and inhibited whole-body lipolysis as evidenced by the decrease in plasma glycerol and FFA concentrations ${ }^{143}$.

Thus, both animal and human data show, an antilipolytic effect of acetate that may decrease lipid overflow to peripheral insulin-sensitive tissues (e.g. skeletal muscle) thereby possibly improving insulin sensitivity and decreasing hypothalamic inflammation. 


\subsubsection{Adipogenesis and browning of adipose tissue}

Acetate may affect the proliferation and differentiation of adipocytes, thereby contributing to adipose tissue morphology, browning and function, which can induce high thermogenic activity with the potential to enhance oxidative capacity ${ }^{141}$. Hereby, we discuss adipogenic/browning effects of acetate mainly derived from in vitro and animal data. First, a 7-day acetate incubation $(0.1 \mu \mathrm{mol} / \mathrm{l})$ of 3T3-L1 pre-adipocytes increased mRNA levels of GPR43 and peroxisome proliferator-activated receptor gamma (PPAR $\gamma)$, which is a master regulator of adipogenesis, suggesting a modulatory effect in adipocyte differentiation ${ }^{144,}{ }^{145}$. In support, incubation of immortalized mice brown adipose tissue cells with a supraphysiological concentration of acetate $(10 \mathrm{mmol} / \mathrm{l})$ enhanced mRNA expression levels of $P P A R \gamma$, and the browning markers peroxisome proliferatoractivated receptor-gamma coactivator-1 alpha $(P G C-1 \alpha)$ and uncoupling protein 1 (UCP1) ${ }^{146}$. In another in vitro study in 3T3-L1 pre-adipocytes, acetate $(1 \mathrm{mmol} / \mathrm{l})$ modulated the gene expression profile with an increase in the mRNA levels of $U C P 1, P P A R \alpha$, and PPAR $\gamma$, PR domain containing 16 protein (PRDM16) and cell death-inducing DNA fragmentation factor-a-like effector (CIDEA) as well as in markers of beige adipocytes including transmembrane protein 26 (TMEM26) and T-box protein 1 (TBX1) ${ }^{147}$. Importantly, in vitro effects were replicated in KK-Ay mice after a 16-week oral administration of acetate $(0.12 \mathrm{~g} /$ day $)$, however only in epididymal adipose tissue and not in inguinal or brown adipose tissues ${ }^{147}$.

In line, several animal studies reported comparable effects. For instance, a 16-week oral acetate supplementation ( $5 \% \mathrm{w} / \mathrm{w}$ diet) in HFD-fed mice increased the expressions of several key genes in epididymal adipose tissue possibly mediated via increased GPR41/GPR43 expression. The genes were involved in mitochondrial biogenesis (PGC-1 $\alpha$, nuclear respiratory factor 1, mitochondrial transcription factor a, beta-F1-ATPase, nuclear-encoded subunit IV and cytochrome complex), which were all reduced by the HFD alone ${ }^{87}$. Interestingly, in HFD-fed mice, acetate (injected intraperitoneally in a nanoparticle) increased PRDM16 mRNA expression in white adipose tissue (WAT) and increased thermogenesis ${ }^{141}$.

Recently, intermittent fasting treated mice (every other day fasting, EODF) significantly induced the expression of browning markers (UCP1) in subcutaneous inguinal WAT which was proposed to occur through gut-derived acetate since browning depended on gut microbiota depletion and transplantation ${ }^{61}$. In support, both colonic and serum acetate levels were significantly increased after both shortterm and long-term intermittent fasting ( 3 and 15 cycles of 24 hours, respectively), suggesting an acetate-mediated browning effect ${ }^{61}$. Although human data are scarce, a recent cross-sectional study in morbidly obese individuals reported that elevated circulating acetate levels were positively correlated with mRNA expression of the browning marker PRDM16 in abdominal subcutaneous adipose tissue, and improved insulin sensitivity (2-h euglycemic hyperinsulinemic clamp) and changes in gut microbiota composition (i.e. increased Firmicutes abundance) ${ }^{148}$. In summary, although the human evidence is limited, acetate seems to play a role 
in adipogenesis and browning of WAT and together with the antilipolytic effects, acetate may restructure adipose tissue morphology and improve adipose tissue functioning and energy metabolism and thereby overall metabolic health.

\subsubsection{Adipose tissue inflammation}

Adipose tissue has been recognized as an important modulator of local and systemic low-grade inflammation, which is often associated to the pathophysiology of obesity and development of insulin resistance ${ }^{149}$. Therefore, metabolic cues from the adipose tissue can increase the recruitment, infiltration and activation of immune cells that can promote a proinflammatory secretory profile (low-grade inflammation) ${ }^{150}$. A pro-inflammatory secretory profile is partly characterized by increased pro inflammatory macrophages (M1), less anti-inflammatory macrophages (M2) and T-cell polarization ${ }^{151}$. Here, we discuss acetate-mediated effects on gut-derived metabolic endotoxemia and local adipose tissue inflammation.

Under different pathological conditions, a leaky gut may occur and result in metabolic endotoxemia, characterized by high circulating lipopolysaccharide (LPS) levels which can potentially lead to chronic low-grade inflammation, as often observed in obesity and insulin resistance ${ }^{152}$. Gut-derived acetate may affect gut health via an improvement in intestinal barrier function through cross-feeding mechanisms (i.e. increased butyrate concentration) ${ }^{153}$. In an in vitro study, acetate (30 mmol/l) decreased LPS-stimulated secretion of tumor necrosis factor (TNF- $\alpha$ ) from human neutrophils by 33\% $(\mathrm{P}<0.01)^{154}$.

Furthermore, microbially-derived acetate from specific bifidobacteria protected mice against a lethal injection of Escherichia coli O157:H7 that can potentially increase LPS ${ }^{155}$. In a 3-week oral treatment in mice, acetate added to drinking water $(150 \mathrm{mmol} / \mathrm{l})$ increased the number and function of colonic antiinflammatory Treg cells in a GPR43 dependent manner ${ }^{156}$, suggesting a protective and anti-inflammatory role of colonic acetate. Additionally, in vitro data show that acetate has the potential to decrease immune cell infiltration in the adipose tissue. First, incubation of $\mathrm{T}$ cells from mice with supraphysiological concentration of sodium acetate $(5-20 \mathrm{mmol} / \mathrm{l})$ promoted $\mathrm{T}$ cell differentiation towards an antiinflammatory $\mathrm{T}$ cell polarization (both Th1 and Th17), capable to produce antiinflammatory cytokines such as IL-17, interferon $\gamma$ and interleukin 10 (IL-10) ${ }^{157}$. Authors indicated that acetate can bypass the cell surface and regulate cells that have low expression of GPR41/GPR43 ${ }^{157}$. These effects were putatively regulated by the activation of mTOR-S6K pathway independently of GPR41/GPR43, since both receptors are not expressed in T cells ${ }^{68}$.

In other relevant immune cells, acetate $(10 \mathrm{mmol} / \mathrm{l})$ induced TNF- $\alpha$ expression/secretion in M2 but not in M1 macrophages, which showed that acetate may have a different role through the GPR43 receptor ${ }^{158}$. Acetate may also affect CD8 $\mathrm{T}$ cells, which play a regulatory role in the initiation of adipose tissue 
inflammation, putatively through macrophage differentiation, activation and migration ${ }^{159}$. In mice, abundance of systemic acetate in response to bacterial infections was linked to an optimal function of memory CD8 T cells through an enhanced glycolytic rate and acetylation of glyceraldehyde 3-phosphate dehydrogenase (GAPDH) ${ }^{160}$. In summary, acetate may have a potential role to counteract a leaky gut by preserving gut integrity and health and through immunomodulating pro-inflammatory mechanisms. Furthermore, these putative acetate mediated effects on immune cells (M2 macrophages and T cells) may improve adipose tissue remodeling/functioning and consequently its function towards homeostasis.

\subsection{Acetate and skeletal muscle metabolism}

Obesity is characterized by ectopic fat deposition and an altered skeletal muscle glucose and lipid metabolism, which may exacerbate insulin resistance and lead to the development of T2DM ${ }^{161}$. In this section, we discuss the role of acetate on muscle lipid and glucose metabolism in the context of obesity-associated derangements originated by excessive lipid overflow, a systemic low-grade inflammation and ectopic deposition that may increase insulin resistance in the skeletal muscle.

As mentioned above, GPR41/43 are expressed in the human skeletal muscle tissue ${ }^{27}$. Moreover, acetate uptake has been reported in skeletal muscle tissue in rodents ${ }^{34}$ and humans ${ }^{11}$. In animals, acetate uptake increased together with increments in the AMP/ATP ratio ( $2 \mathrm{~min})$ in muscle tissue after oral injection of $10.5 \mathrm{mg} / \mathrm{kg}$ of BW, however, the absorption machinery remains unclear ${ }^{34}$. Intracellularly, acetate may be rapidly assimilated and metabolized through the TCA cycle in the mitochondrial matrix ${ }^{162}$, contribute to acetyl-CoA pool ${ }^{163}$ and/or modulate signaling mechanisms involved in muscle fat oxidation. For instance, an acetate intragastric infusion $(5 \mathrm{ml} / \mathrm{kg} \mathrm{BW})$ versus water infusion during 6 months in obese rats, revealed an increase in AMPK activity ${ }^{34}$. The putative mechanism is possibly through the catalytic activity of acetyl-CoA synthase that produces acetylCoA and increases AMP in the cytosol. Subsequently, this increases AMP/ATP ratio thereby resulting in an increment of AMPK phosphorylation. In addition, myoglobin and glucose transporter 4 (GLUT4) and lipolytic gene expression (LCACD, 3KACT, and PPAR) were upregulated in both the abdominal and the foreleg muscle tissue ${ }^{34}$. Although, no actual substrate utilization was measured, oxygen consumption rate measurements (using metabolic cage) showed $7 \%$ higher rates in acetate-treated rats, indicating possible increase in whole-body metabolic rate following intragastric acetate infusion.

Furthermore, in an in vitro study, using rat skeletal muscle (L6) cells, acetic acid increased the AMP/ATP ratio and the phosphorylation of AMPK in a dose (0.05-0.5 mmol/l) and time (0-30 $\mathrm{min})$ dependent manner using physiological concentrations ${ }^{33}$. With respect to glucose metabolism, acetate supplementation $(0.2$ $\mathrm{g}$ acetic acid/100 $\mathrm{g}$ diet for 2 hours) increased glycogen storage (1.1-fold) and 
decreased glycolysis in the gastrocnemius as compared to control (no acetate) in Sprague-Dawley rats ${ }^{164}$. In support, as mentioned above, increments in the protein expression of GLUT4 ${ }^{34}$ may suggest a modulatory role in glucose homeostasis. In summary, acetate may modulate skeletal muscle lipid and glucose metabolism possibly through activation (phosphorylation) of AMPK. Whether this affects endogenous intramyocellular triacylglycerol (IMTG) and/or exogenous (dietary) fat oxidation and glucose homeostasis in human muscle remains unclear.

\subsection{Acetate and liver metabolism}

Hepatic steatosis, if untreated, may progress towards non-alcoholic steatohepatitis (NASH) and aggravate pathophysiology in obesity and comorbidities. Therefore, adequate treatment to tackle hepatic fat accumulation is of utmost importance. As mentioned above, the liver plays a central role in acetate metabolism, since important endogenous production occurs here. Furthermore, acetate may be rapidly metabolized and used as a carbon donor for intracellular pathways including cholesterol biosynthesis, acetylation processes ${ }^{165}$ and hepatic palmitate formation ${ }^{92}$. Importantly, the liver is the first organ in direct contact with microbially-produced acetate coming from the ileum and proximal colon.

From a mechanistic perspective both in vitro and animal studies have shed light on the role of acetate in liver substrate metabolism. In Fao cells (rat hepatoma cell line), 1-hour incubation with physiological concentrations of sodium acetate (100-200 $\mu \mathrm{mol} / \mathrm{l}$ ) increased AMPK phosphorylation (pThr 172) by $40 \%$ compared to control treated cells ${ }^{35}$. Similarly, after 2-hour oral acetate administration (16.7 $\mathrm{mmol} / \mathrm{l}$ at $10 \mathrm{ml} / \mathrm{kg}$ body weight) in ICR (albino strain) mice, pAMPK and pAMPK/AMPK ratio increased ${ }^{35}$. In the same study, a basic chow diet with acetic acid added to a final concentration of $0.3 \%$ for 8 weeks in KK-A (y) mice showed a hypoglycemic effect, lowering of TAG and increased glycogen content in the liver, whilst AMPK phosphorylation was not significantly increased ${ }^{35}$. In HFD-fed mice, supplementation with low $(0.3 \%)$ and high (1.5\%) acetic acid for 6 weeks decreased hepatic lipid accumulation, liver lipids and increments in expression of hepatic genes associated with fatty acid oxidation (UCP2, PPAR $\alpha, \mathrm{CPT} 1$ and ACOX) ${ }^{88}$.

Furthermore, in HepG2 cells, these effects were ablated when cells were depleted of AMPK using siRNA, suggesting an AMPK mediated mechanism ${ }^{88}$. In line with modulatory role of acetate, intraperitoneal injections in rats (dose of 20 $\mathrm{mmol} / \mathrm{kg}$ body weight) showed an increase in the AMP/ATP ratio in liver extracts after 15 min ${ }^{166}$.

Additionally, in HFD-fed mice, intraperitoneal injection of acetate (nanoparticle delivery method), decreased hepatic lipid accumulation, improved hepatic function and increased mitochondrial efficiency ${ }^{141}$.

Acetate supplementations may also increase hepatic glycogen synthesis in the muscle as reported in rats ${ }^{167,168}$. Of note, the acetate:propionate ratio may be of importance for hepatic lipid biosynthesis since propionate may favor odd chain fatty acids while acetate may favor palmitate formation ${ }^{169}$. Moreover, odd chain fatty acids have been linked to improvements in insulin sensitivity ${ }^{170}$. Moreover, in 
men it has been suggested that propionate may reduce acetate utilization for liver lipid biosynthesis (fatty acid and cholesterol) ${ }^{171}$. However, acetate has shown antilipolytic effects at the whole-body and adipose tissue level and increases in whole-body fat oxidation (See Adipose tissue metabolism). For instance, a 3-hour intragastric infusion of acetate (equivalent to the fermentation of $30 \mathrm{~g}$ of dietary fibers) in healthy subjects decreased plasma FFA, thus possibly improving lipid profiles ${ }^{119}$. Importantly, SCFA reach the liver in different ratios, thus single SCFA may not reflect physiological effects in the lipid profile. In general, in vitro and animal studies have provided mechanistic insight into the role of acetate in the liver, where it may increase the AMP/ATP ratio, subsequently increasing AMPK phosphorylation/activity and thereby affecting hepatic lipid (FA oxidation) and glucose (glycogen) metabolism.

\subsection{Acetate and insulin secretion in beta cells}

Glucose homeostasis is intricately regulated via insulin which is secreted from the beta cells in the pancreas ${ }^{172}$. Therefore, there is no question that modulation of glucose stimulated insulin secretion (GSIS) has an impact on glucose homeostasis ${ }^{173}$. From a mechanistic perspective, murine and human beta cells express GPR (GPR41 and GPR43) ${ }^{29}$, via which acetate has reported to regulate insulin secretion ${ }^{28}$. In support to this G protein-mediated signaling, GPR43 deficient mice show glucose intolerance, and reduced beta cell mass and function 174. Importantly, a study reported that GPR43 signaling pathway may be mediated by divergent $G$ protein pathways that can selectively potentiate $\left(G \alpha_{q} / 11\right.$ signaling can lead to $\mathrm{Ca}^{2+}$ mobilization and enhance GSIS) or inhibit $\left(\mathrm{G} \alpha_{\mathrm{i} / \mathrm{o}}\right.$ signaling can lead to cAMP inhibition and diminish GSIS) insulin secretion in rodents ${ }^{29}$. In addition, acetate at physiological concentrations (70-170 $\mu \mathrm{mol} / \mathrm{l}){ }^{175}$ only increased in vitro insulin secretion in a murine beta cell line but not in human islets and suggested species-specific differences ${ }^{29}$. However, the model to induce insulin exocytosis via $\mathrm{Ca}^{2+}$ mobilization inside the beta cells has been a consensus for a long time, other acetate mediated signals may indirectly contribute to insulin secretion, such as via gut-derived GLP-1 and vagal activation of the parasympathetic nervous system ${ }^{176}$. Of note, GLP-1 may exert a direct effect on beta cells by closing $\mathrm{K}^{+}$ATP channel in a glucose dependent mechanism to stimulate insulin exocytosis 177-179. However, whether acetate enhances gut satiety hormones remains elusive as discussed above. Furthermore, vagal activation increased GSIS in a rat study (see acetate and central effects on appetite regulation) associated with an increased acetate turnover ${ }^{40}$. However, inconsistent results have been reported, since an in vitro study using isolated rat pancreatic islets, showed that acetate $(1 \mathrm{mmol} / \mathrm{l})$ strongly decreased GSIS ${ }^{180}$. Acetate may potentially modulate circulating levels of insulin, however, whether acetate directly affects insulin secretion via $G$ protein-mediated signaling, or indirectly via vagal/parasympathetic activation or gut-derived hormones remains to be elucidated. With respect to alpha cells only very few studies have investigated this, with no clear effects of acetate on glucagon release ${ }^{180,181}$. 
Overall both human acetate colonic infusions and vinegar administrations have reported effects to improve insulin sensitivity and glucose homeostasis. From a mechanistic perspective acetate may modulate improvements in adipose tissue functioning, as well as through an increase in oxidative capacity (i.e. muscle, liver) and modulation of GSIS in the pancreas. Collectively, these tissue specific effects may synergistically decrease lipid ectopic deposition and contribute to body weight control and glucose homeostasis. In the next sections, we discuss the preand probiotic human administrations with potential to increase acetate production.

\section{Prebiotic and probiotic administrations, body weight control and insulin sensitivity}

In this section, we discuss pre- and probiotic administrations to increase microbial-derived acetate and its effects in metabolic health. First, we discuss the effect of acetogenic fiber supplementation in humans. Second, we discuss the potential of probiotic interventions to increase microbial-derived acetate production in the gut and its effects on human metabolic health.

\subsection{Acetogenic fibers in human studies}

As described above, acetogenic fibers in the diet may be fermented in the colon by the microbial community and thereby increase colonic acetate production 182-184. Here we show that acetogenic fibers may increase circulating acetate levels and a few studies have even been linked to improvements in whole-body and peripheral insulin sensitivity (See Table 4). For instance, in healthy individuals, a drink containing lactulose $(0-20 \mathrm{~g})$ showed a dose effect to increase acetate concentrations with the highest dose reaching $>200 \mu \mathrm{mol} / 1$ in plasma after 2.5 hours and even 6 hours after ingestion (>100 $\mu \mathrm{mol} / \mathrm{l})$, however, no metabolic outcome was measured ${ }^{51}$. In line, other acute lactulose studies in healthy individuals reported an increase in whole-body acetate turnover ${ }^{185}$, ${ }^{186}$. In addition, young healthy individuals consuming bread overnight (evening of previous day) supplemented with $18.4 \mathrm{~g}$ arabinoxylan oligosaccharides reported increased plasma acetate levels $(245 \mu \mathrm{mol} / \mathrm{l})$ after $10.5 \mathrm{~h}$ fasting and 2 hours after a standardized breakfast (215 $\mu \mathrm{mol} / 1)^{187}$.

This was accompanied by improved glucose tolerance and insulin sensitivity possibly via increased gut fermentation (as evidenced by increased circulating SCFA and breath hydrogen levels) ${ }^{187}$. In overweight individuals, single oral administration of $24 \mathrm{~g}$ inulin in overnight fasted overweight individuals, increased serum acetate and possibly reduced ghrelin in comparison to glucose and resistant starch as control ${ }^{109}$. A study in overweight subjects, using a single administration of lactulose (30 g) significantly increased plasma acetate (333 $\mu \mathrm{mol} / \mathrm{l})$ in comparison to saline administration $(197 \mu \mathrm{mol} / \mathrm{l}) 6 \mathrm{~h}$ after ingestion. Moreover, acetate turnover correlated negatively with glycerol levels $(r=-0.78$, $\mathrm{p}<0.02)$, and a decrease in free fatty acids (35\%) was observed $2 \mathrm{~h}$ after lactulose 
ingestion ${ }^{188}$. In hypercholesterolemic men, oat bran consumption for 3 weeks showed a total cholesterol lowering effect $(12.8 \%)$ putatively through a greater serum acetate production as compared to wheat bran ${ }^{189}$.

Recently, a high fiber diet (including traditional Chinese medicinal foods and prebiotics) for 12 weeks in T2DM subjects, showed higher improvement in glycemic control (HbA1c $<7 \%$ ) than the control diet (89\% vs 50\%, respectively) ${ }^{190}$. Of note, in this study an active SCFA producer (ASP) index, based on the abundance and diversity of the 15 high-fiber promoted microbial SCFA producers, was increased much more in the high fiber diet as compared to the control diet ${ }^{190}$. Another relevant human study, showed that a resistant starch (30 g RS/d) intervention improved insulin sensitivity (measured with hyperinsulinaemiceuglycemic clamp), lowered circulating lipids (non-esterified fatty acids and glycerol), increased fasting ghrelin and improved insulin sensitivity during a meal tolerance test, which was accompanied by higher AUC for acetate ${ }^{11}$. Despite the beneficial effects of RS, the increments in ghrelin seemed counterintuitive considering the expected higher satiety after RS. Nevertheless, some studies have linked ghrelin with increased insulin sensitivity ${ }^{191,192}$. Although Robertson et al. ${ }^{11}$, found beneficial effects on insulin sensitivity in healthy individuals using the gold standard measurement, the studied population is relatively small $(n=10)$ and other RCT with acetogenic fibers within a larger population did not find beneficial effects (See Table 4) ${ }^{193,}{ }^{194}$. Moreover, a study conducted by Zhao et al. ${ }^{190}$ differed in duration compared to Robertson et al. and according to Zhao et al. results, T2DM subjects may need an integrative intervention, since they reported beneficial effects in the group treated with a fiber rich diet combined with lifestyle intervention and medication (acarbose).

Although the previous studies indicate that acetate may increase in response to acetogenic fiber supplementation and improve certain metabolites, other studies reported no effects of acetogenic fibers on metabolic profile. For instance, a 12-week GOS supplementation (15 g/day) to the regular diet in obese prediabetic individuals showed an increase in fecal Bifidobacterium but no effects on acetate levels (fecal and plasma), insulin sensitivity and energy metabolism ${ }^{193}$.

Similarly, another 12-week GOS supplementation (5.5 g/day) in overweight/prediabetic adults improved gut microbial community (increased Bifidobacterium and lowered Bacteroides) and reduced inflammatory markers (including C-reactive protein and fecal calprotectin), however, acetate was not measured ${ }^{195}$. Taken together, the discrepancies of effects between studies may suggest the importance of the type and dose of dietary fiber as well as the metabolic phenotype studied. In the next section, we discuss the potential of probiotics to improve body weight and insulin sensitivity in humans. 
Table 4. Acetogenic fiber administrations with effects on glucose homeostasis and metabolic health.

\begin{tabular}{|c|c|c|c|c|}
\hline Participants & $\begin{array}{l}\text { Non-digestible } \\
\text { carbohydrate }\end{array}$ & Design & Effects & Study \\
\hline T2DM individuals $(n=43)$ & $\begin{array}{l}\text { High fiber diet } \\
(\mathrm{n}=27) \\
\text { Control diet }(\mathrm{n}=16)\end{array}$ & $\begin{array}{l}12 \text { weeks } \\
\text { RCT }\end{array}$ & $\begin{array}{l}\text { Higher improvement } \\
\text { in glycemic control } \\
(\mathrm{HbA} 1 \mathrm{c} \quad<7 \%) \text { in } \\
\text { treatment }(89 \% \quad \mathrm{vs} \\
50 \% \text {, respectively) } \\
\uparrow \text { abundance in SCFA } \\
\text { microbiota producers } \\
\text { in treatment }\end{array}$ & $\begin{array}{l}\text { Zhao et al. } \\
\text { (2018) } 190\end{array}$ \\
\hline $\begin{array}{l}\text { Healthy adults } \\
(\mathrm{n}=10)\end{array}$ & $\begin{array}{l}20 \mathrm{~g} \text { resistant starch } \\
10 \mathrm{~g} \text { ( } 3 \text { times/day) }\end{array}$ & $\begin{array}{l}4 \quad \text { weeks, } \\
\text { placebo/controlled (20 } \\
\text { g digestible starch) }\end{array}$ & $\begin{array}{l}\text { Improved whole-body } \\
\text { insulin sensitivity } \\
\text { (euglycemic- } \\
\text { hyperinsulinaemic } \\
\text { clamp) by } 13 \% \\
(\mathrm{P}<0.05)\end{array}$ & $\begin{array}{l}\text { Robertson } \\
\text { et al. } \\
(2005))^{11}\end{array}$ \\
\hline $\begin{array}{l}\text { Hypercholesterolemic } \\
\text { men }(n=20)\end{array}$ & $\begin{array}{ll}\text { Oat bran } & (47.4 \\
\text { g/day) } & \text { wheat bran } \\
\text { (control) } & (41.8 \\
\text { g/day) } & \end{array}$ & $\begin{array}{l}\text { RCT } \\
3 \text { weeks }\end{array}$ & $\begin{array}{l}\downarrow \quad \text { Total cholesterol } \\
(12.8 \%) \\
\text { Linked to high acetate } \\
\text { in plasma } \\
\uparrow \text { Higher acetate in } \\
\text { treatment }\end{array}$ & $\begin{array}{l}\text { Bridges et } \\
\text { al. (1992) }{ }^{189}\end{array}$ \\
\hline $\begin{array}{l}\text { Healthy individuals } \\
(\mathrm{n}=14)\end{array}$ & $\begin{array}{l}0,10 \text { and } 20 \mathrm{gr} \\
\text { lactulose } \\
\text { Control (water) }\end{array}$ & Single dose & $\begin{array}{l}\text { No effects in glucose } \\
\text { homeostasis } \\
\text { investigated } \\
\text { Dose effect in acetate } \\
\text { levels } \\
\uparrow \text { fermentation after } 6 \\
\text { hours }\end{array}$ & $\begin{array}{l}\text { Pomare et } \\
\text { al. (1985) }{ }^{51}\end{array}$ \\
\hline $\begin{array}{l}\text { Healthy individuals } \\
(n=19)\end{array}$ & $\begin{array}{l}\text { Arabynoxylan } \\
\text { oligosaccharides } \\
\text { (AXOS) ( } 8.9 \text { gr) } \\
\text { High AXOS (18.4 } \\
\text { gr) }\end{array}$ & $\begin{array}{l}\text { Randomized cross- } \\
\text { over } \\
\text { Overnight } \\
\text { administration }\end{array}$ & $\begin{array}{l}\uparrow \text { Improvement in } \\
\text { glucose tolerance } \\
\uparrow \quad \text { improved insulin } \\
\text { sensitivity index with } \\
\text { High AXOS } \\
\text { Dose-effect increase in } \\
\text { plasma acetate (>200 } \\
\mu \text { mol/l) }\end{array}$ & $\begin{array}{l}\text { Boli et al. } \\
(2016)^{187}\end{array}$ \\
\hline $\begin{array}{l}\text { Overweight } \\
\text { individuals }(n=53)\end{array}$ & $\begin{array}{l}\text { Pea fiber (15 g/day) } \\
\text { Control (no fiber) }\end{array}$ & $\begin{array}{l}\text { RCT } \\
12 \text { weeks }\end{array}$ & $\begin{array}{l}\text { No effects in glucose } \\
\text { homeostasis } \\
\uparrow \text { Fecal acetate } \\
\text { No effects on plasma } \\
\text { acetate }\end{array}$ & $\begin{array}{l}\text { Mayengba } \\
\text { ng et al. } \\
(2017)^{194}\end{array}$ \\
\hline $\begin{array}{l}\text { Lean/overweight } \\
\text { individuals } \quad(n=12, \quad 13 \\
\text { respectively) }\end{array}$ & $\begin{array}{l}\text { Inulin (24 gr) } \\
\text { Control (glucose) }\end{array}$ & $\begin{array}{l}\text { Cross-over } \\
\text { Single dose }\end{array}$ & $\begin{array}{l}\uparrow \text { Acetate in plasma } \\
\text { Possibly linked to } \\
\text { ghrelin reduction }\end{array}$ & $\begin{array}{l}\text { Rahat- } \\
\text { Rozenbloo } \\
\mathrm{m} \text { et al. } \\
(2016)^{109}\end{array}$ \\
\hline $\begin{array}{l}\text { Overweight individuals } \\
(\mathrm{n}=8)\end{array}$ & Lactulose (30 gr) & Single dose & $\begin{array}{l}\uparrow \text { Acetate in plasma } \\
\text { Correlation of acetate } \\
\text { and in lipolysis } \\
\text { (glycerol turnover) }\end{array}$ & $\begin{array}{l}\text { Ferchaud- } \\
\text { Roucher et } \\
\text { al. (2005) }\end{array}$ \\
\hline
\end{tabular}

Abbreviations: T2DM, type 2 diabetes mellitus; RCT, randomized controlled trial; HbA1c, glycated hemoglobin; SCFA, short chain fatty acids. 


\subsection{Probiotic body weight control and insulin sensitivity}

Targeting the gut microbiota with probiotics has gained interest as a therapeutic approach to combat obesity and its comorbidities. Interestingly, in rodents at the genus level, Bifidobacterium and Lactobacillus have been associated with reduced weight gain and markers of adiposity ${ }^{196}$. Here we discuss both animal and human studies on probiotic interventions/associations with putatively acetatemediated metabolic health effects.

First, a probiotic intervention in mice, (Bifidobacterium animalis ssp. lactis GCL2505) modified microbial community (increased Bifidobacterium and Lactobacillus), increased acetate (caecal and plasma) levels and improved glucose tolerance which was accompanied by a reduction of adipocyte cell size ${ }^{13}$. Second, mice colonized with Bifidobacterium strains (Bifidobacterium longum JCM 1217, B. infantis 157F, or B. longum NCC 2705) were protected against a lethal infection of Escherichia coli O157:H7 through an augmented acetate production and Bifidobacterium that promoted gut barrier integrity and reduced epithelial cell death 197. Furthermore, Wrsozek et al. ${ }^{198}$ proposed that two species, including Bacteroides thetaiotaomicron (acetate producer) and Faecalibacterium prausnitzii (acetate consumer), might aid in maintaining epithelial homeostasis and gut health in rats.

Another important bacterium, A. muciniphila, has been associated with improvements in adiposity in both rodents and humans ${ }^{196,199}$ and loss of $A$. muciniphila has been reported to impair gut integrity and increase insulin resistance in rodents ${ }^{200}$. A. muciniphila has been reported to correlate with serum acetate levels in humans, thus, suggesting a putative role of $A$. muciniphila in acetate production and metabolic health ${ }^{63}$. Recently, a RCT reported that pasteurized A. muciniphila supplementation (3 months) in overweight/obese insulin resistant individuals improved insulin sensitivity (HOMA-IR, $+28.62+7.02 \%, \mathrm{P}=0.002)$, decreased body weight $(-2.27+0.92 \mathrm{~kg}, \mathrm{P}=0.091)$, fat mass $(-1.37+0.82, \mathrm{P}=0.092)$ among other relevant blood markers ${ }^{201}$.

Similarly, a 3 month supplementation (L.casei Shirota) improved insulin sensitivity index in metabolic syndrome individuals compared to baseline, but not different with control ${ }^{202}$. Although, these studies showed that the interventions were safe and provided benefits on metabolic health, an in-depth study of the microbial-derived metabolites is needed to identify the metabolic regulators. In addition, as Anhe et al. ${ }^{203}$ suggested, the identification of these metabolites may provide a safer therapeutic option and overcome the limitations of probiotic supplementations. In addition, a metanalysis of randomized controlled trials reported that probiotic supplementation (i.e. Lactobacillus reuteri, Lactobacillus gasseri) can significantly reduce body weight ((95\% CI) $-0.60[-1.19,-0.01] \mathrm{kg}), \mathrm{BMI}$ $\left((95 \% \mathrm{CI})-0.27[-0.45,-0.08] \mathrm{kg} / \mathrm{m}^{2}\right)$ and fat percentage $((95 \% \mathrm{CI})-0.60[-1.20,-0.01]$ $\%)$ in overweight/obese individuals, however, the effect sizes were small ${ }^{204}$. $L$. 
plantarum reduced glucose and homocysteine levels significantly after 12 weeks in premenopausal insulin resistant women ${ }^{205}$. In addition, combination of probiotics with prebiotics may provide a synergistic effect. For instance, a synbiotic administration (Lactobacillus and fructo-oligosaccharides) in insulin resistant individuals showed improvements in fasting levels of glucose and HOMA-IR in comparison to placebo after a 28 -week treatment ${ }^{206}$.

Future probiotic studies should identify the mechanisms, factors that ensure its efficacy as well as which synbiotic mixtures may have a better synergistic effect ${ }^{207,208}$. Although these studies report benefits on insulin sensitivity (HOMAIR) and glucose levels with potential to improve metabolic health following probiotic interventions, it remains unknown whether these effects are acetatemediated. Nevertheless, effects were mediated by specific species such as Bacteroides thetaiotaomicron and A. muciniphila, which are important acetate producers.

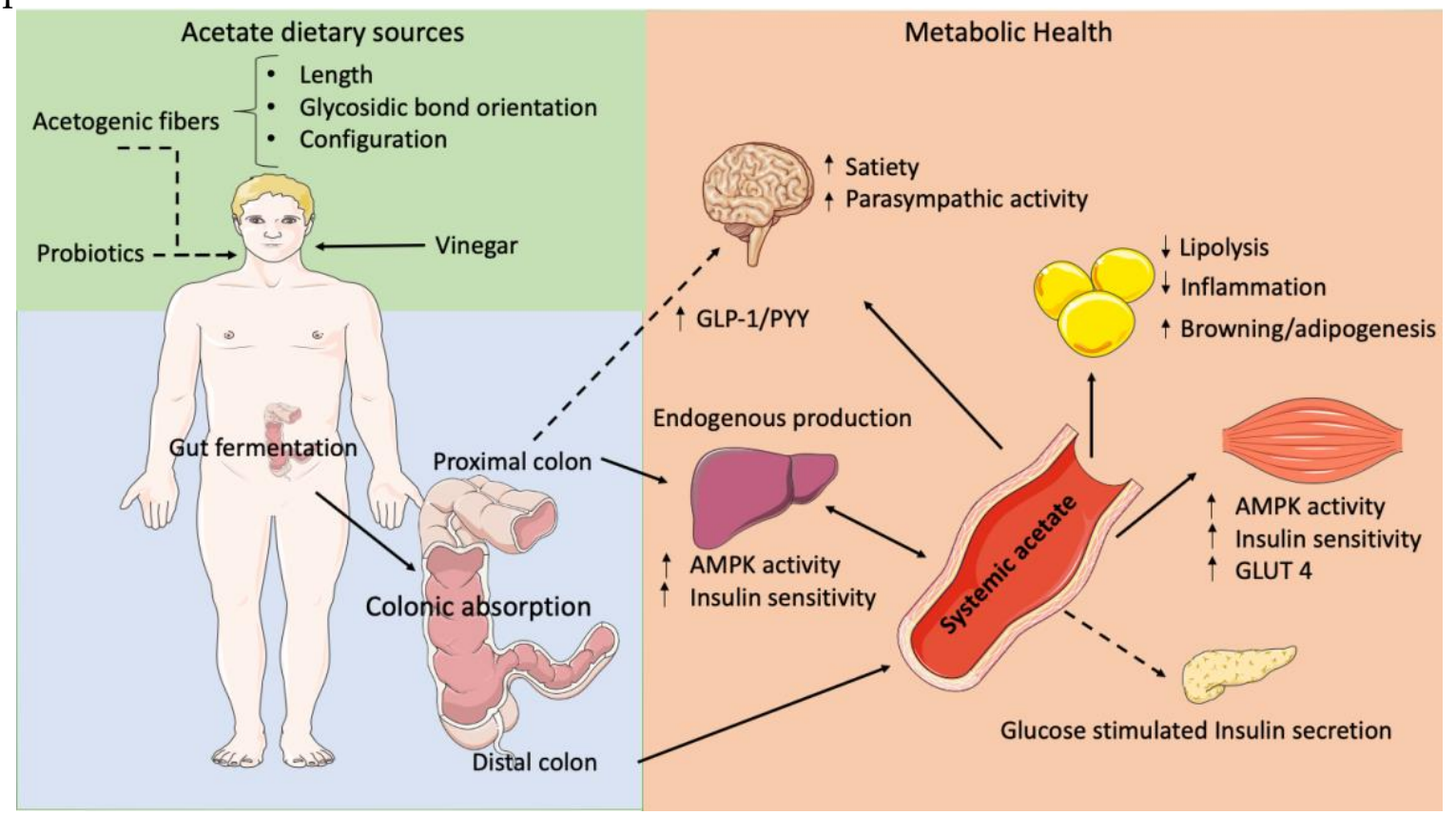

Figure 1: Acetate sources and acetate-mediated effects in metabolic health. Exogenous acetate production includes vinegar as well as the supplementation of acetogenic fiber and probiotics. Acetogenic fiber characteristics (i.e. length, glycosidic bond configuration) may determine acetate production. Endogenous acetate production occurs in all tissues but predominantly in the liver. Microbial acetate is mainly produced in the colon. Colonic absorption and acetate systemic concentrations may differ between colonic production sites (proximal/distal). Importantly, acetate may increase GLP-1 and PYY secretion in the colon. Systemic acetate may improve metabolic health via improvements in adipose tissue functioning (antilipolytic/anti-inflammatory effects) insulin sensitivity and oxidative capacity (i.e. liver, skeletal muscle) increments in satiety (central nervous system) and modulation of insulin secretion (pancreas). Solid lines indicate well-studied effects of acetate, dashed line indicate more inconsistent findings. Abbreviations: GLP-1 glucagonlike peptide 1; PYY peptide YY; AMPK AMP-activated protein kinase; GLUT glucose transporter. 


\section{Conclusions and perspectives}

From a mechanistic perspective, a vast wealth of animal data suggests that acetate has an important regulatory role in body weight control, and insulin sensitivity through effects on lipid metabolism and glucose homeostasis (see Figure 1). Current evidence of acetate-mediated effects on metabolism emphasizes the need for well-controlled human intervention studies that ensure an efficient administration of acetate (considering location and concentration) in a physiological and appealing manner. Under this premise, prebiotic supplementations have been conducted, however with inconsistent results to increase colonic/systemic acetate production. Nevertheless, a few human studies have shown the capacity to improve markers of whole-body insulin sensitivity ${ }^{11}$, 187,190 . Similarly, a few probiotic human studies, with the potential to increase acetate production, have reported improvements in whole-body insulin sensitivity ${ }^{202,206 .}$ While the effect of changes in acetate levels on the observed metabolic phenotype in these studies remains unclear, in light of the evidence presented in this report, it is reasonable to hypothesize that acetate acts as a direct mediator of these effects. With respect to oral acetate administrations (vinegar), improvements on glucose homeostasis have been reported, and attributed to acetic acid. Of importance, future vinegar supplementations should specify its composition (i.e. acetic acid \% percentage, polyphenols). Moreover, future prebiotic/probiotic/vinegar studies should consider that responses may differ between healthy individuals and prediabetic individuals, as evidently shown in vinegar administrations. 


\section{References}

1. Worldwide trends in body-mass index, underweight, overweight, and obesity from 1975 to 2016: a pooled analysis of 2416 population-based measurement studies in 128.9 million children, adolescents, and adults. Lancet. 2017;390(10113):2627-42. Epub 2017/10/17. doi: 10.1016/s0140-6736(17)32129-3. PubMed PMID: 29029897; PubMed Central PMCID: PMCPMC5735219.

2. Smith KB, Smith MS. Obesity Statistics. Prim Care. 2016;43(1):121-35, ix. Epub 2016/02/21. doi: 10.1016/j.pop.2015.10.001. PubMed PMID: 26896205.

3. Mittendorfer B. Origins of metabolic complications in obesity: adipose tissue and free fatty acid trafficking. Curr Opin Clin Nutr Metab Care. 2011;14(6):535-41. Epub 2011/08/19. doi: 10.1097/MCO.0b013e32834ad8b6. PubMed PMID: 21849896; PubMed Central PMCID: PMCPMC3711689.

4. Petersen KF, Shulman GI. Etiology of insulin resistance. Am J Med. 2006;119(5 Suppl 1):S10-S6. doi: 10.1016/j.amjmed.2006.01.009. PubMed PMID: 16563942.

5. Stinkens R, Goossens GH, Jocken JW, Blaak EE. Targeting fatty acid metabolism to improve glucose metabolism. Obes Rev. 2015;16(9):715-57. Epub 2015/07/17. doi: 10.1111/obr.12298. PubMed PMID: 26179344.

6. Schroeder BO, Bäckhed F. Signals from the gut microbiota to distant organs in physiology and disease. Nature Medicine. 2016;22(10):1079-89. doi: 10.1038/nm.4185.

7. DiBaise J, Frank D, Mathur R. Impact of the Gut Microbiota on the Development of Obesity: Current Concepts. The American Journal of Gastroenterology Supplements. 2012;1:22-7. doi: 10.1038/ajgsup.2012.5.

8. Graham C, Mullen A, Whelan K. Obesity and the gastrointestinal microbiota: A review of associations and mechanisms. Nutr Rev. 2015;73. doi: 10.1093/nutrit/nuv004.

9. Reijnders D, Goossens G, Hermes G, Neis E, van der Beek C, Most J, et al. Effects of Gut Microbiota Manipulation by Antibiotics on Host Metabolism in Obese Humans: A Randomized Double-Blind Placebo-Controlled Trial. Cell metabolism. 2016;24:341. doi: 10.1016/j.cmet.2016.07.008.

10. Vrieze A, Out C, Fuentes S, Jonker L, Reuling I, Kootte RS, et al. Impact of oral vancomycin on gut microbiota, bile acid metabolism, and insulin sensitivity. Journal of Hepatology. 2014;60(4):824-31. doi: 10.1016/j.jhep.2013.11.034.

11. Robertson MD, Bickerton AS, Dennis AL, Vidal H, Frayn KN. Insulinsensitizing effects of dietary resistant starch and effects on skeletal muscle and adipose tissue metabolism. Am J Clin Nutr. 2005;82(3):559-67. Epub 2005/09/13. doi: 10.1093/ajcn.82.3.559. PubMed PMID: 16155268.

12. Salden B, Troost F, Wilms E, Truchado P, Vilchez-Vargas R, Pieper D, et al. Reinforcement of intestinal epithelial barrier by arabinoxylans in overweight 
and obese subjects: A randomized controlled trial. Clinical Nutrition. 2017;37. doi: 10.1016/j.clnu.2017.01.024.

13. Aoki R, Kamikado K, Suda W, Takii H, Mikami Y, Suganuma N, et al. A proliferative probiotic Bifidobacterium strain in the gut ameliorates progression of metabolic disorders via microbiota modulation and acetate elevation. Scientific Reports. 2017;7(1):43522. doi: 10.1038/srep43522.

14. Aguilar-Toalá JE, Garcia-Varela R, Garcia HS, Mata-Haro V, GonzálezCórdova AF, Vallejo-Cordoba B, et al. Postbiotics: An evolving term within the functional foods field. Trends in Food Science \& Technology. 2018;75:105-14. doi: 10.1016/j.tifs.2018.03.009.

15. Conlon MA, Bird AR. The Impact of Diet and Lifestyle on Gut Microbiota and Human Health. Nutrients. 2015;7(1):17-44. doi: 10.3390/nu7010017. PubMed PMID: PMC4303825.

16. Canfora EE, Jocken JW, Blaak EE. Short-chain fatty acids in control of body weight and insulin sensitivity. Nat Rev Endocrinol. 2015;11(10):577-91. doi: 10.1038/nrendo.2015.128.

17. Marchesi JR, Adams DH, Fava F, Hermes GDA, Hirschfield GM, Hold G, et al. The gut microbiota and host health: a new clinical frontier. Gut. 2015.

18. Macfarlane GT, Macfarlane S. Bacteria, Colonic Fermentation, and Gastrointestinal Health. Journal of AOAC International. 2012;95(1):50-60. doi: 10.5740/jaoacint.SGE_Macfarlane.

19. Schwiertz A, Taras D, Schafer K, Beijer S, Bos NA, Donus C, et al. Microbiota and SCFA in lean and overweight healthy subjects. Obesity (Silver Spring). 2010;18(1):190-5. Epub 2009/06/06. doi: 10.1038/oby.2009.167. PubMed PMID: 19498350.

20. Fernandes J, Su W, Rahat-Rozenbloom S, Wolever TMS, Comelli EM. Adiposity, gut microbiota and faecal short chain fatty acids are linked in adult humans. Nutrition \& diabetes. 2014;4(6):e121-e. doi: 10.1038/nutd.2014.23. PubMed PMID: 24979150.

21. Asano I, Ikeda Y, Fujii S, Iino H. Effects of Mannooligosaccharides from Coffee on Microbiota and Short Chain Fatty Acids in Rat Cecum. Food Science and Technology Research. 2004;10(3):273-7. doi: 10.3136/fstr.10.273.

22. Cummings JH, Pomare EW, Branch WJ, Naylor CP, Macfarlane GT. Short chain fatty acids in human large intestine, portal, hepatic and venous blood. Gut. 1987;28(10):1221-7. doi: 10.1136/gut.28.10.1221. PubMed PMID: 3678950.

23. Karaki S, Tazoe H, Hayashi H, Kashiwabara H, Tooyama K, Suzuki Y, et al. Expression of the short-chain fatty acid receptor, GPR43, in the human colon. Journal of molecular histology. 2008;39(2):135-42. Epub 2007/09/28. doi: 10.1007/s10735-007-9145-y. PubMed PMID: 17899402.

24. Tazoe H, Otomo Y, Karaki S, Kato I, Fukami Y, Terasaki M, et al. Expression of short-chain fatty acid receptor GPR41 in the human colon. 
Biomedical research (Tokyo, Japan). 2009;30(3):149-56. Epub 2009/07/04. PubMed PMID: 19574715.

25. Nohr MK, Pedersen MH, Gille A, Egerod KL, Engelstoft MS, Husted AS, et al. GPR41/FFAR3 and GPR43/FFAR2 as cosensors for short-chain fatty acids in enteroendocrine cells vs FFAR3 in enteric neurons and FFAR2 in enteric leukocytes. Endocrinology. 2013;154(10):3552-64. Epub 2013/07/26. doi: 10.1210/en.2013-1142. PubMed PMID: 23885020.

26. Le Poul E, Loison C, Struyf S, Springael JY, Lannoy V, Decobecq ME, et al. Functional characterization of human receptors for short chain fatty acids and their role in polymorphonuclear cell activation. J Biol Chem. 2003;278(28):25481-9. Epub 2003/04/25. doi: 10.1074/jbc.M301403200. PubMed PMID: 12711604.

27. Brown AJ, Goldsworthy SM, Barnes AA, Eilert MM, Tcheang L, Daniels D, et al. The Orphan G protein-coupled receptors GPR41 and GPR43 are activated by propionate and other short chain carboxylic acids. J Biol Chem. 2003;278(13):11312-9. Epub 2002/12/24. doi: 10.1074/jbc.M211609200. PubMed PMID: 12496283.

28. Tang C, Ahmed K, Gille A, Lu S, Grone H-J, Tunaru S, et al. Loss of FFA2 and FFA3 increases insulin secretion and improves glucose tolerance in type 2 diabetes. Nat Med. 2015;21(2):173-7. doi: 10.1038/nm.3779.

29. Priyadarshini M, Villa SR, Fuller M, Wicksteed B, Mackay CR, Alquier $\mathrm{T}$, et al. An Acetate-Specific GPCR, FFAR2, Regulates Insulin Secretion. Molecular Endocrinology. 2015;29(7):1055-66. doi: 10.1210/me.2015-1007.

30. Ballard FJ. Supply and utilization of acetate in mammals. Am J Clin Nutr. 1972;25(8):773-9. Epub 1972/08/01. doi: 10.1093/ajcn/25.8.773. PubMed PMID: 4558368.

31. Knowles SE, Jarrett IG, Filsell OH, Ballard FJ. Production and utilization of acetate in mammals. Biochem J. 1974;142(2):401-11. Epub 1974/08/01. doi: 10.1042/bj1420401. PubMed PMID: 4441381; PubMed Central PMCID: PMCPMC1168292.

32. Mittendorfer B, Sidossis LS, Walser E, Chinkes DL, Wolfe RR. Regional acetate kinetics and oxidation in human volunteers. Am J Physiol. 1998;274(6):E978-83. Epub 1998/06/05. doi: 10.1152/ajpendo.1998.274.6.E978. PubMed PMID: 9611145.

33. Maruta H, Yoshimura Y, Araki A, Kimoto M, Takahashi Y, Yamashita H. Activation of AMP-Activated Protein Kinase and Stimulation of Energy Metabolism by Acetic Acid in L6 Myotube Cells. PloS one. 2016;11(6):e0158055e. doi: 10.1371/journal.pone.0158055. PubMed PMID: 27348124.

34. Yamashita H, Maruta H, Jozuka M, Kimura R, Iwabuchi H, Yamato M, et al. Effects of acetate on lipid metabolism in muscles and adipose tissues of type 2 diabetic Otsuka Long-Evans Tokushima Fatty (OLETF) rats. Biosci Biotechnol Biochem. 2009;73(3):570-6. Epub 2009/03/10. doi: 10.1271/bbb.80634. PubMed PMID: 19270372. 
35. Sakakibara S, Yamauchi T, Oshima Y, Tsukamoto Y, Kadowaki T. Acetic acid activates hepatic AMPK and reduces hyperglycemia in diabetic KK-A(y) mice. Biochem Biophys Res Commun. 2006;344(2):597-604. Epub 2006/04/25. PubMed PMID: 16630552.

36. Gao X, Lin S-H, Ren F, Li J-T, Chen J-J, Yao C-B, et al. Acetate functions as an epigenetic metabolite to promote lipid synthesis under hypoxia. Nature Communications. 2016;7:11960. doi: 10.1038/ncomms11960.

37. Pluznick JL, Protzko RJ, Gevorgyan H, Peterlin Z, Sipos A, Han J, et al. Olfactory receptor responding to gut microbiota-derived signals plays a role in renin secretion and blood pressure regulation. Proceedings of the National Academy of Sciences of the United States of America. 2013;110(11):4410-5. doi: 10.1073/pnas.1215927110. PubMed PMID: PMC3600440.

38. Bindels Laure B, Leclercq I. Colonic acetate in obesity: location matters! Clinical Science. 2016;130(22):2083-6. doi: 10.1042/cs20160556.

39. van der Beek CM, Canfora EE, Lenaerts K, Troost FJ, Damink SW, Holst JJ, et al. Distal, not proximal, colonic acetate infusions promote fat oxidation and improve metabolic markers in overweight/obese men. Clin Sci (Lond). 2016;130(22):2073-82. Epub 2016/07/22. doi: 10.1042/cs20160263. PubMed PMID: 27439969.

40. Trajkovski M, Wollheim CB. Physiology: Microbial signals to the brain control weight. Nature. 2016;534(7606):185-7. doi: 10.1038/534185a.

41. Canfora EE, Blaak EE. Acetate: a diet-derived key metabolite in energy metabolism: good or bad in context of obesity and glucose homeostasis? Curr Opin Clin Nutr Metab Care. 2017. Epub 2017/08/11. doi: 10.1097/mco.0000000000000408. PubMed PMID: 28795972.

42. Kasselman LJ, Vernice NA, DeLeon J, Reiss AB. The gut microbiome and elevated cardiovascular risk in obesity and autoimmunity. Atherosclerosis. 2018;271:203-13. Epub 2018/03/11. doi: 10.1016/j.atherosclerosis.2018.02.036. PubMed PMID: 29524863.

43. Aron-Wisnewsky J, Clement K. The gut microbiome, diet, and links to cardiometabolic and chronic disorders. Nat Rev Nephrol. 2016;12(3):169-81. Epub 2015/12/01. doi: 10.1038/nrneph.2015.191. PubMed PMID: 26616538.

44. Iwabuchi H. Codex alimentarius Commission Codex General standard for food additives. Joint FAO/WHO [Internet]. 2016 31/01/2017.

45. Israel Y, Orrego H, Carmichael FJ. Acetate-mediated effects of ethanol. Alcoholism, clinical and experimental research. 1994;18(1):144-8. Epub 1994/02/01. PubMed PMID: 8198211.

46. Lim J, Henry CJ, Haldar S. Vinegar as a functional ingredient to improve postprandial glycemic control-human intervention findings and molecular mechanisms. Molecular Nutrition \& Food Research. 2016;60(8):1837-49. doi: 10.1002/mnfr.201600121.

47. Sugiyama S, Fushimi T, Kishi M, Irie S, Tsuji S, Hosokawa N, et al. Bioavailability of acetate from two vinegar supplements: capsule and drink. 
Journal of nutritional science and vitaminology. 2010;56(4):266-9. Epub 2010/10/07. PubMed PMID: 20924150.

48. Budak NH, Aykin E, Seydim AC, Greene AK, Guzel-Seydim ZB. Functional properties of vinegar. J Food Sci. 2014;79(5):R757-64. Epub 2014/05/09. doi: 10.1111/1750-3841.12434. PubMed PMID: 24811350.

49. Ho CW, Lazim AM, Fazry S, Zaki UKHH, Lim SJ. Varieties, production, composition and health benefits of vinegars: A review. Food Chemistry. 2017;221:1621-30. doi: 10.1016/j.foodchem.2016.10.128.

50. Wong JMW, de Souza R, Kendall CWC, Emam A, Jenkins DJA. Colonic Health: Fermentation and Short Chain Fatty Acids. Journal of Clinical Gastroenterology. 2006;40(3):235-43. PubMed PMID: 00004836-20060300000015.

51. Pomare EW, Branch WJ, Cummings JH. Carbohydrate fermentation in the human colon and its relation to acetate concentrations in venous blood. Journal of Clinical Investigation. 1985;75(5):1448-54. PubMed PMID: PMC425482.

52. Schug ZT, Vande Voorde J, Gottlieb E. The metabolic fate of acetate in cancer. Nat Rev Cancer. 2016;16(11):708-17. doi: 10.1038/nrc.2016.87.

53. Ragsdale SW, Pierce E. Acetogenesis and the Wood-Ljungdahl Pathway of CO(2) Fixation. Biochimica et biophysica acta. 2008;1784(12):1873-98. doi: 10.1016/j.bbapap.2008.08.012. PubMed PMID: PMC2646786.

54. Koh A, De Vadder F, Kovatcheva-Datchary P, Bäckhed F. From Dietary Fiber to Host Physiology: Short-Chain Fatty Acids as Key Bacterial Metabolites. Cell. 2016;165(6):1332-45. doi: 10.1016/j.cell.2016.05.041.

55. Mortensen PB, Holtug K, Bonnen H, Clausen MR. The degradation of amino acids, proteins, and blood to short-chain fatty acids in colon is prevented by lactulose. Gastroenterology. 1990;98(2):353-60. Epub 1990/02/01. PubMed PMID: 2295390.

56. Macfarlane GT, Gibson GR, Beatty E, Cummings JH. Estimation of short-chain fatty acid production from protein by human intestinal bacteria based on branched-chain fatty acid measurements. FEMS Microbiology Letters. 1992;101(2):81-8. doi: 10.1111/j.1574-6968.1992.tb05764.x.

57. Perry RJ, Peng L, Barry NA, Cline GW, Zhang D, Cardone RL, et al. Acetate mediates a microbiome-brain- $\beta$-cell axis to promote metabolic syndrome. Nature. 2016;534(7606):213-7. doi: 10.1038/nature18309.

58. Blachier F, Mariotti F, Huneau JF, Tome D. Effects of amino acidderived luminal metabolites on the colonic epithelium and physiopathological consequences. Amino acids. 2007;33(4):547-62. Epub 2006/12/06. doi: 10.1007/s00726-006-0477-9. PubMed PMID: 17146590.

59. Windey K, De Preter V, Verbeke K. Relevance of protein fermentation to gut health. Mol Nutr Food Res. 2012;56(1):184-96. Epub 2011/11/29. doi: 10.1002/mnfr.201100542. PubMed PMID: 22121108. 
60. Canfora EE, Meex RCR, Venema K, Blaak EE. Gut microbial metabolites in obesity, NAFLD and T2DM. Nature Reviews Endocrinology. 2019;15(5):26173. doi: 10.1038/s41574-019-0156-z.

61. Li G, Xie C, Lu S, Nichols RG, Tian Y, Li L, et al. Intermittent Fasting Promotes White Adipose Browning and Decreases Obesity by Shaping the Gut Microbiota. Cell metabolism. 2017;26(4):672-85.e4. Epub 2017/09/19. doi: 10.1016/j.cmet.2017.08.019. PubMed PMID: 28918936; PubMed Central PMCID: PMCPMC5668683.

62. Remely M, Hippe B, Geretschlaeger I, Stegmayer S, Hoefinger I, Haslberger A. Increased gut microbiota diversity and abundance of Faecalibacterium prausnitzii and Akkermansia after fasting: a pilot study. Wiener Klinische Wochenschrift. 2015;127(9-10):394-8. doi: 10.1007/s00508-0150755-1. PubMed PMID: PMC4452615.

63. Dao MC, Everard A, Aron-Wisnewsky J, Sokolovska N, Prifti E, Verger $\mathrm{EO}$, et al. Akkermansia muciniphila and improved metabolic health during a dietary intervention in obesity: relationship with gut microbiome richness and ecology. Gut. 2016;65(3):426-36. Epub 2015/06/24. doi: 10.1136/gutjnl-2014308778. PubMed PMID: 26100928.

64. Neis EPJG, van Eijk HMH, Lenaerts K, Olde Damink SWM, Blaak EE, Dejong $\mathrm{CHC}$, et al. Distal versus proximal intestinal short-chain fatty acid release in man. Gut. 2018.

65. Scheppach W, Pomare EW, Elia M, Cummings JH. The contribution of the large intestine to blood acetate in man. Clin Sci (Lond). 1991;80(2):177-82. Epub 1991/02/01. PubMed PMID: 1848171.

66. Shubitowski TB, Poll BG, Natarajan N, Pluznick JL. Short-chain fatty acid delivery: assessing exogenous administration of the microbiome metabolite acetate in mice. Physiol Rep. 2019;7(4):e14005-e. doi: 10.14814/phy2.14005. PubMed PMID: 30810289.

67. Piekarska J, Miśta D, Houszka M, Króliczewska B, Zawadzki W, Gorczykowski M. Trichinella spiralis: the influence of short chain fatty acids on the proliferation of lymphocytes, the goblet cell count and apoptosis in the mouse intestine. Exp Parasitol. 2011;128(4):419-26. Epub 2011/06/02. doi: 10.1016/j.exppara.2011.05.019. PubMed PMID: 21627965.

68. Kim MH, Kang SG, Park JH, Yanagisawa M, Kim CH. Short-chain fatty acids activate GPR41 and GPR43 on intestinal epithelial cells to promote inflammatory responses in mice. Gastroenterology. 2013;145(2):396-406.e1-10. Epub 2013/05/15. doi: 10.1053/j.gastro.2013.04.056. PubMed PMID: 23665276.

69. Maslowski KM, Vieira AT, Ng A, Kranich J, Sierro F, Di Y, et al. Regulation of inflammatory responses by gut microbiota and chemoattractant receptor GPR43. Nature. 2009;461(7268):1282-6. doi: 10.1038/nature08530.

70. Lebet V, Arrigoni E, Amado R. Digestion Procedure Using Mammalian Enzymes to Obtain Substrates forIn VitroFermentation Studies. LWT - Food Science and Technology. 1998;31(6):509-15. doi: 10.1006/fstl.1998.0402. 
71. Kaur A, Rose DJ, Rumpagaporn P, Patterson JA, Hamaker BR. In Vitro Batch Fecal Fermentation Comparison of Gas and Short-Chain Fatty Acid Production Using "Slowly Fermentable" Dietary Fibers. Journal of Food Science. 2011;76(5):H137-H42. doi: 10.1111/j.1750-3841.2011.02172.x.

72. Maathuis AJH, van den Heuvel EG, Schoterman MHC, Venema K. Galacto-Oligosaccharides Have Prebiotic Activity in a Dynamic In Vitro Colon Model Using a 13C-Labeling Technique. The Journal of Nutrition. 2012;142(7):1205-12. doi: 10.3945/jn.111.157420.

73. Stewart ML, Timm DA, Slavin JL. Fructooligosaccharides exhibit more rapid fermentation than long-chain inulin in an in vitro fermentation system. Nutrition Research. 2008;28(5):329-34. doi: 10.1016/j.nutres.2008.02.014.

74. van de Wiele T, Boon N, Possemiers S, Jacobs H, Verstraete W. Inulintype fructans of longer degree of polymerization exert more pronounced in vitro prebiotic effects. Journal of applied microbiology. 2007;102(2):452-60. Epub 2007/01/24. doi: 10.1111/j.1365-2672.2006.03084.x. PubMed PMID: 17241351.

75. Hamaker BR, Tuncil YE. A perspective on the complexity of dietary fiber structures and their potential effect on the gut microbiota. J Mol Biol. 2014;426(23):3838-50. doi: 10.1016/j.jmb.2014.07.028. PubMed PMID: 25088686.

76. Harris H, Edwards C, Morrison D. Impact of Glycosidic Bond Configuration on Short Chain Fatty Acid Production from Model Fermentable Carbohydrates by the Human Gut Microbiota. Nutrients. 2017;9(1):26. PubMed PMID: doi:10.3390/nu9010026.

77. Tuncil YE, Nakatsu CH, Kazem AE, Arioglu-Tuncil S, Reuhs B, Martens EC, et al. Delayed utilization of some fast-fermenting soluble dietary fibers by human gut microbiota when presented in a mixture. Journal of Functional Foods. 2017;32:347-57. doi: 10.1016/j.jff.2017.03.001.

78. Morrison DJ, Preston T. Formation of short chain fatty acids by the gut microbiota and their impact on human metabolism. Gut Microbes. 2016;7(3):189-200. doi: 10.1080/19490976.2015.1134082.

79. Samuel BS, Gordon JI. A humanized gnotobiotic mouse model of hostarchaeal-bacterial mutualism. Proceedings of the National Academy of Sciences of the United States of America. 2006;103(26):10011-6. Epub 06/16. doi: 10.1073/pnas.0602187103. PubMed PMID: 16782812.

80. Stearns JC, Lynch MDJ, Senadheera DB, Tenenbaum HC, Goldberg MB, Cvitkovitch DG, et al. Bacterial biogeography of the human digestive tract. Scientific Reports. 2011;1:170. doi: 10.1038/srep00170.

81. Suez J, Zmora N, Zilberman-Schapira G, Mor U, Dori-Bachash M, Bashiardes S, et al. Post-Antibiotic Gut Mucosal Microbiome Reconstitution Is Impaired by Probiotics and Improved by Autologous FMT. Cell. 2018;174(6):1406-23.e16. doi: 10.1016/j.cell.2018.08.047.

82. Van Herreweghen F, Van den Abbeele P, De Mulder T, De Weirdt R, Geirnaert A, Hernandez-Sanabria E, et al. In vitro colonisation of the distal 
colon by Akkermansia muciniphila is largely mucin and $\mathrm{pH}$ dependent. Benef Microbes. 2017;8(1):81-96. Epub 2016/11/09. doi: 10.3920/bm2016.0013. PubMed PMID: 27824274.

83. Derrien M, van Baarlen P, Hooiveld G, Norin E, Muller M, de Vos W. Modulation of Mucosal Immune Response, Tolerance, and Proliferation in Mice Colonized by the Mucin-Degrader Akkermansia muciniphila. Front Microbiol. 2011;2(166). doi: 10.3389/fmicb.2011.00166.

84. Preidis GA, Ajami NJ, Wong MC, Bessard BC, Conner ME, Petrosino JF. Composition and function of the undernourished neonatal mouse intestinal microbiome. J Nutr Biochem. 2015;26(10):1050-7. Epub 2015/06/14. doi: 10.1016/j.jnutbio.2015.04.010. PubMed PMID: 26070414.

85. Geerlings SY, Kostopoulos I, de Vos WM, Belzer C. Akkermansia muciniphila in the Human Gastrointestinal Tract: When, Where, and How? Microorganisms. 2018;6(3). Epub 2018/07/26. doi: 10.3390/microorganisms6030075. PubMed PMID: 30041463.

86. den Besten G, Bleeker A, Gerding A, van Eunen K, Havinga R, van Dijk $\mathrm{TH}$, et al. Short-Chain Fatty Acids Protect Against High-Fat Diet-Induced Obesity via a PPARgamma-Dependent Switch From Lipogenesis to Fat Oxidation. Diabetes. 2015;64(7):2398-408. Epub 2015/02/20. doi: 10.2337/db141213. PubMed PMID: 25695945.

87. Lu Y, Fan C, Li P, Lu Y, Chang X, Qi K. Short Chain Fatty Acids Prevent High-fat-diet-induced Obesity in Mice by Regulating G Protein-coupled Receptors and Gut Microbiota. Scientific Reports. 2016;6:37589. doi: 10.1038/srep37589.

88. Kondo T, Kishi M, Fushimi T, Kaga T. Acetic Acid Upregulates the Expression of Genes for Fatty Acid Oxidation Enzymes in Liver To Suppress Body Fat Accumulation. Journal of Agricultural and Food Chemistry. 2009;57(13):5982-6. doi: 10.1021/jf900470c.

89. Frost G, Sleeth ML, Sahuri-Arisoylu M, Lizarbe B, Cerdan S, Brody L, et al. The short-chain fatty acid acetate reduces appetite via a central homeostatic mechanism. Nat Commun. 2014;5:3611. Epub 2014/05/02. doi: 10.1038/ncomms4611. PubMed PMID: 24781306; PubMed Central PMCID: PMCPMC4015327.

90. Falk Petersen K, Impellizeri A, Cline G, I. Shulman G. The effects of increased acetate turnover on glucose-induced insulin secretion in lean and obese humans2019. 1-3 p.

91. Layden BT, Yalamanchi SK, Wolever TM, Dunaif A, Lowe WL, Jr. Negative association of acetate with visceral adipose tissue and insulin levels. Diabetes, metabolic syndrome and obesity : targets and therapy. 2012;5:49-55. Epub 2012/03/16. doi: 10.2147/dmso.S29244. PubMed PMID: 22419881; PubMed Central PMCID: PMCPMC3299553.

92. Goffredo M, Mass K, Parks EJ, Wagner DA, McClure EA, Graf J, et al. Role of Gut Microbiota and Short Chain Fatty Acids in Modulating Energy 
Harvest and Fat Partitioning in Youth. The Journal of clinical endocrinology and metabolism. 2016;101(11):4367-76. Epub 2016/11/05. doi: 10.1210/jc.20161797. PubMed PMID: 27648960; PubMed Central PMCID: PMCPMC5095239.

93. Dhillo WS. Appetite regulation: an overview. Thyroid : official journal of the American Thyroid Association. 2007;17(5):433-45. Epub 2007/06/05. doi: 10.1089/thy.2007.0018. PubMed PMID: 17542673.

94. Jiang L, Gulanski BI, De Feyter HM, Weinzimer SA, Pittman B, Guidone $\mathrm{E}$, et al. Increased brain uptake and oxidation of acetate in heavy drinkers. J Clin Invest. 2013;123(4):1605-14. Epub 2013/03/08. doi: 10.1172/JCI65153. PubMed PMID: 23478412.

95. Nagashima H, Morio Y, Meshitsuka S, Yamane K, Nanjo Y, Teshima R. High-resolution nuclear magnetic resonance spectroscopic study of metabolites in the cerebrospinal fluid of patients with cervical myelopathy and lumbar radiculopathy. European Spine Journal. 2010;19(8):1363-8. doi: 10.1007/s00586010-1453-3. PubMed PMID: PMC2989206.

96. Hu Z, Cha SH, Chohnan S, Lane MD. Hypothalamic malonyl-CoA as a mediator of feeding behavior. Proc Natl Acad Sci U S A. 2003;100(22):12624-9. Epub 2003/10/09. doi: 10.1073/pnas.1834402100. PubMed PMID: 14532332; PubMed Central PMCID: PMCPMC240668.

97. He W, Lam TK, Obici S, Rossetti L. Molecular disruption of hypothalamic nutrient sensing induces obesity. Nat Neurosci. 2006;9(2):227-33. Epub 2006/01/18. doi: 10.1038/nn1626. PubMed PMID: 16415870.

98. Mithieux G. Nutrient control of hunger by extrinsic gastrointestinal neurons. Trends in Endocrinology \& Metabolism. 24(8):378-84. doi: 10.1016/j.tem.2013.04.005.

99. Goswami C, Iwasaki Y, Yada T. Short chain fatty acids suppress food intake by activating vagal afferent neurons. The Journal of Nutritional Biochemistry. 2018. doi: 10.1016/j.jnutbio.2018.03.009.

100. Plamboeck A, Veedfald S, Deacon CF, Hartmann B, Wettergren A, Svendsen LB, et al. The effect of exogenous GLP-1 on food intake is lost in male truncally vagotomized subjects with pyloroplasty. American journal of physiology Gastrointestinal and liver physiology. 2013;304(12):G1117-27. Epub 2013/04/20. doi: 10.1152/ajpgi.00035.2013. PubMed PMID: 23599045.

101. Ahima RS, Antwi DA. Brain regulation of appetite and satiety. Endocrinol Metab Clin North Am. 2008;37(4):811-23. doi: 10.1016/j.ecl.2008.08.005. PubMed PMID: PMC2710609.

102. Gutierrez-Aguilar R, Woods SC. Nutrition and L and Kenteroendocrine cells. Current opinion in endocrinology, diabetes, and obesity. 2011;18(1):10.1097/MED.0b013e32834190b5. doi: 10.1097/MED.0b013e32834190b5. PubMed PMID: PMC3884637.

103. Kasubuchi M, Hasegawa S, Hiramatsu T, Ichimura A, Kimura I. Dietary Gut Microbial Metabolites, Short-chain Fatty Acids, and Host Metabolic 
Regulation. Nutrients. 2015;7(4):2839-49. doi: 10.3390/nu7042839. PubMed PMID: PMC4425176.

104. Zhou J, Martin RJ, Tulley RT, Raggio AM, McCutcheon KL, Shen L, et al. Dietary resistant starch upregulates total GLP-1 and PYY in a sustained daylong manner through fermentation in rodents. American Journal of Physiology - Endocrinology And Metabolism. 2008;295(5):E1160.

105. Brooks L, Viardot A, Tsakmaki A, Stolarczyk E, Howard JK, Cani PD, et al. Fermentable carbohydrate stimulates FFAR2-dependent colonic PYY cell expansion to increase satiety. Mol Metab. 2017;6(1):48-60. Epub 2017/01/27. doi: 10.1016/j.molmet.2016.10.011. PubMed PMID: 28123937; PubMed Central PMCID: PMCPMC5220466.

106. Keenan MJ, Zhou J, McCutcheon KL, Raggio AM, Bateman HG, Todd $\mathrm{E}$, et al. Effects of resistant starch, a non-digestible fermentable fiber, on reducing body fat. Obesity (Silver Spring). 2006;14(9):1523-34. Epub 2006/10/13. doi: 10.1038/oby.2006.176. PubMed PMID: 17030963.

107. Cani PD, Dewever C, Delzenne NM. Inulin-type fructans modulate gastrointestinal peptides involved in appetite regulation (glucagon-like peptide-1 and ghrelin) in rats. British Journal of Nutrition. 2004;92(3):521-6. doi: 10.1079/BJN20041225.

108. Massimino SP, McBurney MI, Field CJ, Thomson ABR, Keelan M, Hayek MG, et al. Fermentable Dietary Fiber Increases GLP-1 Secretion and Improves Glucose Homeostasis Despite Increased Intestinal Glucose Transport Capacity in Healthy Dogs. The Journal of Nutrition. 1998;128(10):1786-93.

109. Rahat-Rozenbloom S, Fernandes J, Cheng J, Wolever TMS. Acute increases in serum colonic short-chain fatty acids elicited by inulin do not increase GLP-1 or PYY responses but may reduce ghrelin in lean and overweight humans. Eur J Clin Nutr. 2016. doi: 10.1038/ejcn.2016.249.

110. Parnell JA, Reimer RA. Weight loss during oligofructose supplementation is associated with decreased ghrelin and increased peptide YY in overweight and obese adults. The American Journal of Clinical Nutrition. 2009;89(6):1751-9. doi: 10.3945/ajcn.2009.27465.

111. Pedersen C, Lefevre S, Peters V, Patterson M, Ghatei MA, Morgan LM, et al. Gut hormone release and appetite regulation in healthy non-obese participants following oligofructose intake. A dose-escalation study. Appetite. 2013;66:44-53. doi: 10.1016/j.appet.2013.02.017.

112. Freeland KR, Wolever TMS. Acute effects of intravenous and rectal acetate on glucagon-like peptide-1, peptide $Y Y$, ghrelin, adiponectin and tumour necrosis factor-alpha. The British journal of nutrition. 2010;103(3):4606. doi: 10.1017/s0007114509991863. PubMed PMID: 19818198.

113. Fernandes J, Vogt J, Wolever TM. Intravenous acetate elicits a greater free fatty acid rebound in normal than hyperinsulinaemic humans. Eur J Clin Nutr. 2012;66(9):1029-34. Epub 2012/07/26. doi: 10.1038/ejcn.2012.98. PubMed PMID: 22828730; PubMed Central PMCID: PMCPMC3937122. 
114. Nilsson OLA, Bilchik AJ, Goldenring JR, Ballantyne GH, Adrian TE, Modlin IM. Distribution and Immunocytochemical Colocalization of Peptide YY and Enteroglucagon in Endocrine Cells of the Rabbit Colon*. Endocrinology. 1991;129(1):139-48. doi: 10.1210/endo-129-1-139.

115. Arantes RM, Nogueira AM. Distribution of enteroglucagon- and peptide YY-immunoreactive cells in the intestinal mucosa of germ-free and conventional mice. Cell and tissue research. 1997;290(1):61-9. Epub 1997/09/11. PubMed PMID: 9377643.

116. Xiong Y, Miyamoto N, Shibata K, Valasek MA, Motoike T, Kedzierski $\mathrm{RM}$, et al. Short-chain fatty acids stimulate leptin production in adipocytes through the G protein-coupled receptor GPR41. Proc Natl Acad Sci U S A. 2004;101(4):1045-50. Epub 2004/01/15. doi: 10.1073/pnas.2637002100. PubMed PMID: 14722361; PubMed Central PMCID: PMCPMC327148.

117. Soliman M, Kimura K, Ahmed M, Yamaji D, Matsushita Y, OkamatsuOgura $Y$, et al. Inverse regulation of leptin mRNA expression by short- and long-chain fatty acids in cultured bovine adipocytes. Domestic animal endocrinology. 2007;33(4):400-9. Epub 2006/10/03. doi: 10.1016/j.domaniend.2006.08.005. PubMed PMID: 17011156.

118. Wolever TM, Brighenti F, Royall D, Jenkins AL, Jenkins DJ. Effect of rectal infusion of short chain fatty acids in human subjects. Am J Gastroenterol. 1989;84(9):1027-33. Epub 1989/09/01. PubMed PMID: 2773895.

119. Laurent C, Simoneau C, Marks L, Braschi S, Champ M, Charbonnel B, et al. Effect of acetate and propionate on fasting hepatic glucose production in humans. Eur J Clin Nutr. 1995;49(7):484-91. Epub 1995/07/01. PubMed PMID: 7588498 .

120. Canfora EE, van der Beek CM, Jocken JWE, Goossens GH, Holst JJ, Olde Damink SWM, et al. Colonic infusions of short-chain fatty acid mixtures promote energy metabolism in overweight/obese men: a randomized crossover trial. Scientific Reports. 2017;7(1):2360. doi: 10.1038/s41598-017-02546-x.

121. Akanji AO, Bruce MA, Frayn KN. Effect of acetate infusion on energy expenditure and substrate oxidation rates in non-diabetic and diabetic subjects. Eur J Clin Nutr. 1989;43(2):107-15. Epub 1989/02/01. PubMed PMID: 2651106.

122. Kondo T, Kishi M, Fushimi T, Ugajin S, Kaga T. Vinegar intake reduces body weight, body fat mass, and serum triglyceride levels in obese Japanese subjects. Biosci Biotechnol Biochem. 2009;73(8):1837-43. Epub 2009/08/08. PubMed PMID: 19661687.

123. Kim EK, An SY, Lee MS, Kim TH, Lee HK, Hwang WS, et al. Fermented kimchi reduces body weight and improves metabolic parameters in overweight and obese patients. Nutrition research (New York, NY). 2011;31(6):436-43. Epub 2011/07/13. doi: 10.1016/j.nutres.2011.05.011. PubMed PMID: 21745625.

124. Han K, Bose S, Wang JH, Kim BS, Kim MJ, Kim EJ, et al. Contrasting effects of fresh and fermented kimchi consumption on gut microbiota composition and gene expression related to metabolic syndrome in obese 
Korean women. Mol Nutr Food Res. 2015;59(5):1004-8. Epub 2015/02/18. doi: 10.1002/mnfr.201400780. PubMed PMID: 25688926.

125. Ley RE, Turnbaugh PJ, Klein S, Gordon JI. Microbial ecology: human gut microbes associated with obesity. Nature. 2006;444(7122):1022-3. Epub 2006/12/22. doi: 10.1038/4441022a. PubMed PMID: 17183309.

126. Beh BK, Mohamad NE, Yeap SK, Ky H, Boo SY, Chua JYH, et al. Antiobesity and anti-inflammatory effects of synthetic acetic acid vinegar and Nipa vinegar on high-fat-diet-induced obese mice. Scientific Reports. 2017;7(1):6664. doi: 10.1038/s41598-017-06235-7.

127. Saunders DR. Absorption of short-chain fatty acids in human stomach and rectum. Nutrition Research. 1991;11(8):841-7. doi: 10.1016/S02715317(05)80612-8.

128. Shim S-M, Kim JY, Lee SM, Park J-B, Oh S-K, Kim Y-S. Profiling of fermentative metabolites in kimchi: Volatile and non-volatile organic acids. Journal of the Korean Society for Applied Biological Chemistry. 2012;55(4):4639. doi: 10.1007/s13765-012-2014-8.

129. Choe SS, Huh JY, Hwang IJ, Kim JI, Kim JB. Adipose Tissue Remodeling: Its Role in Energy Metabolism and Metabolic Disorders. Front Endocrinol (Lausanne). 2016;7:30. doi: 10.3389/fendo.2016.00030. PubMed PMID: PMC4829583.

130. Liljeberg $\mathrm{H}$, Bjorck I. Delayed gastric emptying rate may explain improved glycaemia in healthy subjects to a starchy meal with added vinegar. Eur J Clin Nutr. 1998;52(5):368-71. Epub 1998/06/18. PubMed PMID: 9630389.

131. Brighenti F, Castellani G, Benini L, Casiraghi MC, Leopardi E, Crovetti $\mathrm{R}$, et al. Effect of neutralized and native vinegar on blood glucose and acetate responses to a mixed meal in healthy subjects. Eur J Clin Nutr. 1995;49(4):2427. Epub 1995/04/01. PubMed PMID: 7796781.

132. Östman E, Granfeldt Y, Persson L, Björck I. Vinegar supplementation lowers glucose and insulin responses and increases satiety after a bread meal in healthy subjects. European Journal Of Clinical Nutrition. 2005;59:983. doi: 10.1038/sj.ejcn.1602197.

133. Leeman M, Ostman E, Bjorck I. Vinegar dressing and cold storage of potatoes lowers postprandial glycaemic and insulinaemic responses in healthy subjects. Eur J Clin Nutr. 2005;59(11):1266-71. Epub 2005/07/22. doi: 10.1038/sj.ejcn.1602238. PubMed PMID: 16034360.

134. Mitrou P, Petsiou E, Papakonstantinou E, Maratou E, Lambadiari V, Dimitriadis $\mathrm{P}$, et al. The role of acetic acid on glucose uptake and blood flow rates in the skeletal muscle in humans with impaired glucose tolerance. Eur J Clin Nutr. 2015;69(6):734-9. Epub 2015/01/30. doi: 10.1038/ejcn.2014.289. PubMed PMID: 25626409.

135. Liatis S, Grammatikou S, Poulia KA, Perrea D, Makrilakis K, Diakoumopoulou E, et al. Vinegar reduces postprandial hyperglycaemia in patients with type II diabetes when added to a high, but not to a low, glycaemic 
index meal. Eur J Clin Nutr. 2010;64(7):727-32. Epub 2010/05/27. doi: 10.1038/ejcn.2010.89. PubMed PMID: 20502468.

136. van Dijk JW, Tummers K, Hamer HM, van Loon LJ. Vinegar coingestion does not improve oral glucose tolerance in patients with type 2 diabetes. Journal of diabetes and its complications. 2012;26(5):460-1. Epub 2012/06/16. doi: 10.1016/j.jdiacomp.2012.05.009. PubMed PMID: 22699111.

137. Ge H, Li X, Weiszmann J, Wang P, Baribault H, Chen J-L, et al. Activation of G Protein-Coupled Receptor 43 in Adipocytes Leads to Inhibition of Lipolysis and Suppression of Plasma Free Fatty Acids. Endocrinology. 2008;149(9):4519-26. doi: 10.1210/en.2008-0059.

138. Aberdein N, Schweizer M, Ball D. Sodium acetate decreases phosphorylation of hormone sensitive lipase in isoproterenol-stimulated 3T3L1 mature adipocytes. Adipocyte. 2014;3(2):121-5. doi: 10.4161/adip.27936. PubMed PMID: PMC3979876.

139. Heimann E, Nyman M, Pålbrink A-K, Lindkvist-Petersson K, Degerman E. Branched short-chain fatty acids modulate glucose and lipid metabolism in primary adipocytes. Adipocyte. 2016;5(4):359-68. doi: 10.1080/21623945.2016.1252011. PubMed PMID: 27994949.

140. Jocken JWE, González Hernández MA, Hoebers NTH, van der Beek CM, Essers YPG, Blaak EE, et al. Short-Chain Fatty Acids Differentially Affect Intracellular Lipolysis in a Human White Adipocyte Model. Front Endocrinol (Lausanne). 2018;8(372). doi: 10.3389/fendo.2017.00372.

141. Sahuri-Arisoylu M, Brody LP, Parkinson JR, Parkes H, Navaratnam N, Miller AD, et al. Reprogramming of hepatic fat accumulation and 'browning' of adipose tissue by the short-chain fatty acid acetate. International journal of obesity (2005). 2016;40(6):955-63. Epub 2016/03/16. doi: 10.1038/ijo.2016.23. PubMed PMID: 26975441.

142. Crouse JR, Gerson CD, DeCarli LM, Lieber CS. Role of acetate in the reduction of plasma free fatty acids produced by ethanol in man. J Lipid Res. 1968;9(4):509-12.

143. Suokas A, Kupari M, Heikkila J, Lindros K, Ylikahri R. Acute cardiovascular and metabolic effects of acetate in men. Alcoholism, clinical and experimental research. 1988;12(1):52-8. Epub 1988/02/01. PubMed PMID: 3279860.

144. Hong YH, Nishimura Y, Hishikawa D, Tsuzuki H, Miyahara H, Gotoh $\mathrm{C}$, et al. Acetate and propionate short chain fatty acids stimulate adipogenesis via GPCR43. Endocrinology. 2005;146(12):5092-9. Epub 2005/08/27. doi: 10.1210/en.2005-0545. PubMed PMID: 16123168.

145. Siersbaek R, Nielsen R, Mandrup S. PPARgamma in adipocyte differentiation and metabolism--novel insights from genome-wide studies. FEBS letters. 2010;584(15):3242-9. Epub 2010/06/15. doi: 10.1016/j.febslet.2010.06.010. PubMed PMID: 20542036. 
146. Hu J, Kyrou I, Tan BK, Dimitriadis GK, Ramanjaneya M, Tripathi G, et al. Short-Chain Fatty Acid Acetate Stimulates Adipogenesis and Mitochondrial Biogenesis via GPR43 in Brown Adipocytes. Endocrinology. 2016;157(5):188194. doi: 10.1210/en.2015-1944.

147. Hanatani S, Motoshima H, Takaki Y, Kawasaki S, Igata M, Matsumura $\mathrm{T}$, et al. Acetate alters expression of genes involved in beige adipogenesis in 3T3-L1 cells and obese KK-Ay mice. J Clin Biochem Nutr. 2016;59(3):207-14. Epub 2016/11/30. doi: 10.3164/jcbn.16-23. PubMed PMID: 27895388; PubMed Central PMCID: PMCPMC5110936.

148. Moreno-Navarrete JM, Serino M, Blasco-Baque V, Azalbert V, Barton $\mathrm{RH}$, Cardellini M, et al. Gut Microbiota Interacts with Markers of Adipose Tissue Browning, Insulin Action and Plasma Acetate in Morbid Obesity. Mol Nutr Food Res. 2017. Epub 2017/11/07. doi: 10.1002/mnfr.201700721. PubMed PMID: 29105287.

149. Huh JY, Park YJ, Ham M, Kim JB. Crosstalk between Adipocytes and Immune Cells in Adipose Tissue Inflammation and Metabolic Dysregulation in Obesity. Molecules and Cells. 2014;37(5):365-71. doi: 10.14348/molcells.2014.0074. PubMed PMID: PMC4044307.

150. Ferrante AW. The Immune Cells in Adipose Tissue. Diabetes, obesity \& metabolism. 2013;15(0 3):34-8. doi: 10.1111/dom.12154. PubMed PMID: PMC3777665.

151. Guzik TJ, Skiba DS, Touyz RM, Harrison DG. The role of infiltrating immune cells in dysfunctional adipose tissue. Cardiovascular research. 2017;113(9):1009-23. Epub 2017/08/26. doi: 10.1093/cvr/cvx108. PubMed PMID: 28838042; PubMed Central PMCID: PMCPMC5852626.

152. Cani PD, Amar J, Iglesias MA, Poggi M, Knauf C, Bastelica D, et al. Metabolic endotoxemia initiates obesity and insulin resistance. Diabetes. 2007;56(7):1761-72. Epub 2007/04/26. doi: 10.2337/db06-1491. PubMed PMID: 17456850 .

153. Duncan SH, Holtrop G, Lobley GE, Calder AG, Stewart CS, Flint HJ. Contribution of acetate to butyrate formation by human faecal bacteria. British Journal of Nutrition. 2004;91(6):915-23. Epub 2007/03/09. doi: 10.1079/BJN20041150.

154. Tedelind S, Westberg F, Kjerrulf M, Vidal A. Anti-inflammatory properties of the short-chain fatty acids acetate and propionate: a study with relevance to inflammatory bowel disease. World journal of gastroenterology. 2007;13(20):2826-32. Epub 2007/06/15. PubMed PMID: 17569118; PubMed Central PMCID: PMCPMC4395634.

155. Fukuda S, Toh H, Hase K, Oshima K, Nakanishi Y, Yoshimura K, et al. Bifidobacteria can protect from enteropathogenic infection through production of acetate. Nature. 2011;469(7331):543-7. Epub 2011/01/29. doi: 10.1038/nature09646. PubMed PMID: 21270894. 
156. Smith PM, Howitt MR, Panikov N, Michaud M, Gallini CA, Bohlooly YM, et al. The microbial metabolites, short-chain fatty acids, regulate colonic Treg cell homeostasis. Science. 2013;341(6145):569-73. Epub 2013/07/06. doi: 10.1126/science.1241165. PubMed PMID: 23828891; PubMed Central PMCID: PMCPMC3807819.

157. Park J, Kim M, Kang SG, Jannasch AH, Cooper B, Patterson J, et al. Short chain fatty acids induce both effector and regulatory $\mathrm{T}$ cells by suppression of histone deacetylases and regulation of the mTOR-S6K pathway. Mucosal immunology. 2015;8(1):80-93. doi: 10.1038/mi.2014.44. PubMed PMID: PMC4263689.

158. Nakajima A, Nakatani A, Hasegawa S, Irie J, Ozawa K, Tsujimoto G, et al. The short chain fatty acid receptor GPR43 regulates inflammatory signals in adipose tissue M2-type macrophages. PLoS One. 2017;12(7):e0179696-e. doi: 10.1371/journal.pone.0179696. PubMed PMID: 28692672.

159. Nishimura S, Manabe I, Nagasaki M, Eto K, Yamashita H, Ohsugi M, et al. CD8+ effector $\mathrm{T}$ cells contribute to macrophage recruitment and adipose tissue inflammation in obesity. Nat Med. 2009;15(8):914-20. Epub 2009/07/28. doi: 10.1038/nm.1964. PubMed PMID: 19633658.

160. Balmer Maria L, Ma Eric H, Bantug Glenn R, Grählert J, Pfister S, Glatter $\mathrm{T}$, et al. Memory CD8+ T Cells Require Increased Concentrations of Acetate Induced by Stress for Optimal Function. Immunity. 2016;44(6):1312-24. doi: 10.1016/j.immuni.2016.03.016.

161. DeFronzo RA, Tripathy D. Skeletal Muscle Insulin Resistance Is the Primary Defect in Type 2 Diabetes. Diabetes Care. 2009;32(Suppl 2):S157-S63. doi: 10.2337/dc09-S302. PubMed PMID: PMC2811436.

162. Itsuki-Yoneda A, Kimoto M, Tsuji H, Hiemori M, Yamashita H. Effect of a hypolipidemic drug, Di (2-ethylhexyl) phthalate, on mRNA-expression associated fatty acid and acetate metabolism in rat tissues. Biosci Biotechnol Biochem. 2007;71(2):414-20. Epub 2007/02/08. doi: 10.1271/bbb.60478. PubMed PMID: 17284823.

163. Bertocci LA, Jones JG, Malloy CR, Victor RG, Thomas GD. Oxidation of lactate and acetate in rat skeletal muscle: analysis by 13C-nuclear magnetic resonance spectroscopy. Journal of applied physiology (Bethesda, Md : 1985). 1997;83(1):32-9. Epub 1997/07/01. doi: 10.1152/jappl.1997.83.1.32. PubMed PMID: 9216941.

164. Fushimi T, Tayama K, Fukaya M, Kitakoshi K, Nakai N, Tsukamoto Y, et al. Acetic acid feeding enhances glycogen repletion in liver and skeletal muscle of rats. J Nutr. 2001;131(7):1973-7. Epub 2001/07/04. PubMed PMID: 11435516.

165. Yamashita H, Kaneyuki T, Tagawa K. Production of acetate in the liver and its utilization in peripheral tissues. Biochimica et Biophysica Acta (BBA) Molecular and Cell Biology of Lipids. 2001;1532(1):79-87. doi: 10.1016/S13881981(01)00117-2. 
166. Zydowo MM, Smolenski RT, Swierczynski J. Acetate-induced changes of adenine nucleotide levels in rat liver. Metabolism. 1993;42(5):644-8. Epub 1993/05/01. PubMed PMID: 8492721.

167. Nakao C, Yamada E, Fukaya M, Tayama K, Tsukamoto Y, Sato Y. Effect of acetate on glycogen replenishment in liver and skeletal muscles after exhaustive swimming in rats. Scandinavian journal of medicine \& science in sports. 2001;11(1):33-7. Epub 2001/02/13. PubMed PMID: 11169233.

168. Fushimi T, Sato Y. Effect of acetic acid feeding on the circadian changes in glycogen and metabolites of glucose and lipid in liver and skeletal muscle of rats. Br J Nutr. 2005;94(5):714-9. Epub 2005/11/10. PubMed PMID: 16277773.

169. Weitkunat K, Schumann S, Nickel D, Kappo KA, Petzke KJ, Kipp AP, et al. Importance of propionate for the repression of hepatic lipogenesis and improvement of insulin sensitivity in high-fat diet-induced obesity. Mol Nutr Food Res. 2016;60(12):2611-21. Epub 2016/07/29. doi: 10.1002/mnfr.201600305. PubMed PMID: 27467905; PubMed Central PMCID: PMCPMC5215627.

170. Weitkunat K, Schumann S, Nickel D, Hornemann S, Petzke KJ, Schulze $\mathrm{MB}$, et al. Odd-chain fatty acids as a biomarker for dietary fiber intake: a novel pathway for endogenous production from propionate. The American Journal of Clinical Nutrition. 2017;105(6):1544-51. doi: 10.3945/ajcn.117.152702.

171. Wolever TM, Fernandes J, Rao AV. Serum acetate:propionate ratio is related to serum cholesterol in men but not women. J Nutr. 1996;126(11):27907. Epub 1996/11/01. doi: 10.1093/jn/126.11.2790. PubMed PMID: 8914950.

172. Röder PV, Wu B, Liu Y, Han W. Pancreatic regulation of glucose homeostasis. Experimental \& Molecular Medicine. 2016;48(3):e219. doi: 10.1038/emm.2016.6. PubMed PMID: PMC4892884.

173. Cavaghan MK, Ehrmann DA, Polonsky KS. Interactions between insulin resistance and insulin secretion in the development of glucose intolerance. Journal of Clinical Investigation. 2000;106(3):329-33. PubMed PMID: PMC314336.

174. McNelis JC, Lee YS, Mayoral R, van der Kant R, Johnson AMF, Wollam J, et al. GPR43 Potentiates $\beta$-Cell Function in Obesity. Diabetes. 2015;64(9):320317. doi: 10.2337/db14-1938. PubMed PMID: PMC4542437.

175. Layden BT, Angueira AR, Brodsky M, Durai V, Lowe WL, Jr. Short chain fatty acids and their receptors: new metabolic targets. Translational research : the journal of laboratory and clinical medicine. 2013;161(3):131-40. Epub 2012/11/14. doi: 10.1016/j.trsl.2012.10.007. PubMed PMID: 23146568.

176. Tokarz VL, MacDonald PE, Klip A. The cell biology of systemic insulin function. The Journal of Cell Biology. 2018.

177. Meloni AR, DeYoung MB, Lowe C, Parkes DG. GLP-1 receptor activated insulin secretion from pancreatic beta-cells: mechanism and glucose dependence. Diabetes Obes Metab. 2013;15(1):15-27. Epub 2012/07/11. doi: 10.1111/j.1463-1326.2012.01663.x. PubMed PMID: 22776039; PubMed Central PMCID: PMCPMC3556522. 
178. Zander M, Madsbad S, Madsen JL, Holst JJ. Effect of 6-week course of glucagon-like peptide 1 on glycaemic control, insulin sensitivity, and beta-cell function in type 2 diabetes: a parallel-group study. Lancet. 2002;359(9309):82430. Epub 2002/03/19. doi: 10.1016/s0140-6736(02)07952-7. PubMed PMID: 11897280.

179. Meneilly GS, Greig N, Tildesley H, Habener JF, Egan JM, Elahi D. Effects of 3 months of continuous subcutaneous administration of glucagon-like peptide 1 in elderly patients with type 2 diabetes. Diabetes Care. 2003;26(10):2835-41. Epub 2003/09/30. PubMed PMID: 14514588.

180. Tiengo A, Valerio A, Molinari M, Meneghel A, Lapolla A. Effect of ethanol, acetaldehyde, and acetate on insulin and glucagon secretion in the perfused rat pancreas. Diabetes. 1981;30(9):705-9. Epub 1981/09/01. PubMed PMID: 7021270.

181. Sano H, Nakamura E, Takahashi H, Terashima Y. Plasma insulin and glucagon responses to acute challenges of acetate, propionate, n-butyrate and glucose in growing gilts (Sus scrofa). Comp Biochem Physiol A Physiol. 1995;110(4):375-8. Epub 1995/04/01. PubMed PMID: 7735905.

182. David LA, Maurice CF, Carmody RN, Gootenberg DB, Button JE, Wolfe $\mathrm{BE}$, et al. Diet rapidly and reproducibly alters the human gut microbiome. Nature. 2014;505(7484):559-63. doi: 10.1038/nature12820.

183. Titgemeyer EC, Bourquin LD, Fahey GC, Jr., Garleb KA. Fermentability of various fiber sources by human fecal bacteria in vitro. Am J Clin Nutr. 1991;53(6):1418-24. Epub 1991/06/11. PubMed PMID: 1852091.

184. Scott KP, Duncan SH, Flint HJ. Dietary fibre and the gut microbiota. Nutrition Bulletin. 2008;33(3):201-11. doi: 10.1111/j.1467-3010.2008.00706.x.

185. Pouteau E, Vahedi K, Messing B, Flourié B, Nguyen P, Darmaun D, et al. Production rate of acetate during colonic fermentation of lactulose: a stableisotope study in humans. Am J Clin Nutr. 1998;68(6):1276-83. Epub 1998/12/10. doi: 10.1093/ajcn/68.6.1276. PubMed PMID: 9846859.

186. Luo J, Rizkalla SW, Alamowitch C, Boussairi A, Blayo A, Barry JL, et al. Chronic consumption of short-chain fructooligosaccharides by healthy subjects decreased basal hepatic glucose production but had no effect on insulinstimulated glucose metabolism. Am J Clin Nutr. 1996;63(6):939-45. Epub 1996/06/01. doi: 10.1093/ajcn/63.6.939. PubMed PMID: 8644690.

187. Boll EV, Ekstrom LM, Courtin CM, Delcour JA, Nilsson AC, Bjorck IM, et al. Effects of wheat bran extract rich in arabinoxylan oligosaccharides and resistant starch on overnight glucose tolerance and markers of gut fermentation in healthy young adults. Eur J Nutr. 2016;55(4):1661-70. Epub 2015/07/15. doi: 10.1007/s00394-015-0985-z. PubMed PMID: 26169871.

188. Ferchaud-Roucher V, Pouteau E, Piloquet H, Zaïr Y, Krempf M. Colonic fermentation from lactulose inhibits lipolysis in overweight subjects. Am J Physiol Endocrinol Metab. 2005;289(4):E716-20. Epub 2005/09/10. doi: 10.1152/ajpendo.00430.2004. PubMed PMID: 16150956. 
189. Bridges SR, Anderson JW, Deakins DA, Dillon DW, Wood CL. Oat bran increases serum acetate of hypercholesterolemic men. The American Journal of Clinical Nutrition. 1992;56(2):455-9.

190. Zhao L, Zhang F, Ding X, Wu G, Lam YY, Wang X, et al. Gut bacteria selectively promoted by dietary fibers alleviate type 2 diabetes. Science. 2018;359(6380):1151-6. Epub 2018/03/29. doi: 10.1126/science.aao5774. PubMed PMID: 29590046.

191. Ikezaki A, Hosoda H, Ito K, Iwama S, Miura N, Matsuoka H, et al. Fasting plasma ghrelin levels are negatively correlated with insulin resistance and PAI-1, but not with leptin, in obese children and adolescents. Diabetes. 2002;51(12):3408-11. Epub 2002/11/28. doi: 10.2337/diabetes.51.12.3408. PubMed PMID: 12453893.

192. Pagotto U, Gambineri A, Vicennati V, Heiman ML, Tschop M, Pasquali R. Plasma ghrelin, obesity, and the polycystic ovary syndrome: correlation with insulin resistance and androgen levels. The Journal of clinical endocrinology and metabolism. 2002;87(12):5625-9. Epub 2002/12/06. doi: 10.1210/jc.2002020776. PubMed PMID: 12466363.

193. Canfora EE, van der Beek CM, Hermes GDA, Goossens GH, Jocken JWE, Holst JJ, et al. Supplementation of Diet With Galacto-oligosaccharides Increases Bifidobacteria, but Not Insulin Sensitivity, in Obese Prediabetic Individuals. Gastroenterology. 2017;153(1):87-97.e3. Epub 2017/04/12. doi: 10.1053/j.gastro.2017.03.051. PubMed PMID: 28396144.

194. Mayengbam S, Lambert JE, Parnell JA, Tunnicliffe JM, Han J, Sturzenegger $\mathrm{T}$, et al. Dietary Fiber Supplementation Normalizes Serum Metabolites of Adults with Overweight/Obesity in a 12-Week Randomized Control Trial. The FASEB Journal. 2017;31(1 Supplement):433.5.

195. Vulevic J, Juric A, Tzortzis G, Gibson GR. A mixture of transgalactooligosaccharides reduces markers of metabolic syndrome and modulates the fecal microbiota and immune function of overweight adults. J Nutr. 2013;143(3):324-31. Epub 2013/01/11. doi: 10.3945/jn.112.166132. PubMed PMID: 23303873.

196. Mulders RJ, de Git KCG, Schele E, Dickson SL, Sanz Y, Adan RAH. Microbiota in obesity: interactions with enteroendocrine, immune and central nervous systems. Obes Rev. 2018;19(4):435-51. Epub 2018/01/25. doi: 10.1111/obr.12661. PubMed PMID: 29363272.

197. Fukuda S, Toh H, Taylor TD, Ohno H, Hattori M. Acetate-producing bifidobacteria protect the host from enteropathogenic infection via carbohydrate transporters. Gut Microbes. 2012;3(5):449-54. doi: 10.4161/gmic.21214.

198. Wrzosek L, Miquel S, Noordine ML, Bouet S, Joncquel Chevalier-Curt M, Robert V, et al. Bacteroides thetaiotaomicron and Faecalibacterium prausnitzii influence the production of mucus glycans and the development of goblet cells in the colonic epithelium of a gnotobiotic model rodent. BMC 
biology. 2013;11:61. Epub 2013/05/23. doi: 10.1186/1741-7007-11-61. PubMed PMID: 23692866; PubMed Central PMCID: PMCPMC3673873.

199. Everard A, Belzer C, Geurts L, Ouwerkerk JP, Druart C, Bindels LB, et al. Cross-talk between \&lt;em\&gt;Akkermansia muciniphila\&lt;/em\&gt; and intestinal epithelium controls diet-induced obesity. Proceedings of the National Academy of Sciences. 2013;110(22):9066.

200. Bodogai M, O'Connell J, Kim K, Kim Y, Moritoh K, Chen C, et al. Commensal bacteria contribute to insulin resistance in aging by activating innate B1a cells. Science Translational Medicine. 2018;10(467):eaat4271. doi: 10.1126/scitranslmed.aat4271.

201. Depommier C, Everard A, Druart C, Plovier H, Van Hul M, Vieira-Silva $S$, et al. Supplementation with Akkermansia muciniphila in overweight and obese human volunteers: a proof-of-concept exploratory study. Nature Medicine. 2019. doi: 10.1038/s41591-019-0495-2.

202. Tripolt NJ, Leber B, Blattl D, Eder M, Wonisch W, Scharnagl H, et al. Short communication: Effect of supplementation with Lactobacillus casei Shirota on insulin sensitivity, beta-cell function, and markers of endothelial function and inflammation in subjects with metabolic syndrome--a pilot study. Journal of dairy science. 2013;96(1):89-95. Epub 2012/11/21. doi: 10.3168/jds.2012-5863. PubMed PMID: 23164226.

203. Anhê FF, Schertzer JD, Marette A. Bacteria to alleviate metabolic syndrome. Nature Medicine. 2019;25(7):1031-3. doi: 10.1038/s41591-019-0516-1. 204. Borgeraas H, Johnson LK, Skattebu J, Hertel JK, Hjelmesæth J. Effects of probiotics on body weight, body mass index, fat mass and fat percentage in subjects with overweight or obesity: a systematic review and meta-analysis of randomized controlled trials. Obesity Reviews. 2018;19(2):219-32. doi: 10.1111/obr.12626.

205. Barreto FM, Colado Simao AN, Morimoto HK, Batisti Lozovoy MA, Dichi I, Helena da Silva Miglioranza L. Beneficial effects of Lactobacillus plantarum on glycemia and homocysteine levels in postmenopausal women with metabolic syndrome. Nutrition. 2014;30(7-8):939-42. Epub 2014/03/13. doi: 10.1016/j.nut.2013.12.004. PubMed PMID: 24613434.

206. Eslamparast T, Zamani F, Hekmatdoost A, Sharafkhah M, Eghtesad S, Malekzadeh $\mathrm{R}$, et al. Effects of synbiotic supplementation on insulin resistance in subjects with the metabolic syndrome: a randomised, double-blind, placebocontrolled pilot study. Br J Nutr. 2014;112(3):438-45. Epub 2014/05/23. doi: 10.1017/s0007114514000919. PubMed PMID: 24848793.

207. Valdés L, Salazar N, González S, Arboleya S, Ríos-Covián D, Genovés $S$, et al. Selection of potential probiotic bifidobacteria and prebiotics for elderly by using in vitro faecal batch cultures. European Food Research and Technology. 2017;243(1):157-65. doi: 10.1007/s00217-016-2732-y. 
208. Marco ML, Tachon S. Environmental factors influencing the efficacy of probiotic bacteria. Current opinion in biotechnology. 2013;24(2):207-13. Epub 2012/10/30. doi: 10.1016/j.copbio.2012.10.002. PubMed PMID: 23102489. 



\section{CHAPTER 3}

\section{Relation between circulating acetate and human insulin resistance before and after weight loss in the DiOGenes study}

Running title: Acetate and insulin resistance

Manuel A. González Hernández, Emanuel E. Canfora, Kenneth Pasmans, Arne Astrup, Wim H. M. Saris and Ellen E. Blaak.

Nutrients. 2020 Jan; 12 (2):339 


\begin{abstract}
Microbially-produced acetate has been reported to beneficially affect metabolic health through effects on satiety, energy expenditure, insulin sensitivity and substrate utilization. Here, we investigated the association between sex-specific concentrations of acetate and insulin sensitivity/resistance indices (HOMA-IR, circulating insulin and Matsuda Index) in the DiOGenes study at baseline and after a low-calorie diet (LCD, $800 \mathrm{kcal} / \mathrm{d})$. In this analysis 478 subjects $\left(\mathrm{BMI}>27 \mathrm{~kg} / \mathrm{m}^{2}\right)$ were included who underwent a LCD for 8 weeks. Linear mixed models were performed, which were adjusted for mean acetate concentration, center (random factor), age, weight loss, and fat-free mass (FFM). At baseline no associations between plasma acetate and insulin sensitivity/resistance indices were found. We found a slight positive association between changes in acetate and changes in HOMA-IR $(\operatorname{std} \beta 0.130, P=0.033)$ in women, but not in men $(\operatorname{std} \beta-0.072, P=0.310)$ independently of age, weight loss and FFM. We were not able to confirm previously reported associations between acetate and insulin sensitivity in this large European cohort. The mechanisms behind the sex-specific relationship between LCD-induced changes in acetate and insulin sensitivity require further study.
\end{abstract}




\section{Introduction}

Microbially-derived acetate has been shown to play an important role in substrate and energy metabolism ${ }^{1}$. In rodents fed high-fat diets, beneficial effects on insulin sensitivity ${ }^{2}$ and increased white adipose tissue browning were reported ${ }^{3}$. However, rodent data are not entirely consistent since intragastric acetate infusions in rats resulted in hyperphagia and energy retention ${ }^{4}$.

In humans, rectal acetate infusions have been shown to increase plasma peptide YY (PYY) and glucagon-like peptide-1 in hyperinsulinaemic overweight women ${ }^{5}$. Furthermore, acute distal colonic acetate infusions and SCFA mixtures high in acetate decreased whole-body lipolysis, increased fasting fat oxidation, resting energy expenditure and PYY secretion in overweight men 6,7. Additionally, circulating acetate was positively associated with increased fat oxidation and energy expenditure 7. Recently, a study reported that acetate produced in the distal colon contributes significantly to systemic acetate concentrations in men ${ }^{8}$. In line, cross-sectional analyses in patients with obesity have shown positive associations between high circulating acetate and insulin sensitivity indices 9, 10. In women, plasma acetate correlated negatively with fasting and postprandial insulin concentrations ${ }^{9}$. In morbidly obese subjects, acetate correlated negatively with insulin resistance (HOMA-IR) ${ }^{10}$. Notably, a resistant starch supplementation (30 g/d for 4 weeks) in healthy subjects increased acetate uptake in muscle and adipose tissue and improved insulin sensitivity (as measured by hyperinsulinemic-euglycemic clamp) ${ }^{11}$.

Here, we assessed the association of (changes in) circulating acetate and insulin sensitivity/resistance indices (HOMA-IR, circulating insulin and Matsuda Index) at baseline and after an eight weeks low calorie diet (LCD) in male and female overweight/obese subjects of the European Diet, Obesity and Genes (DiOGenes) study. Due to the fact that microbial composition and functionality as well as their impact on insulin sensitivity may differ in overweight males and females, we stratified our analysis for $\operatorname{sex}^{12}$.

\section{Materials and methods}

\subsection{Study Design}

The DiOGenes project was a multicenter, randomized, controlled dietary intervention study in 8 European countries. In total, 938 overweight or obese, nondiabetic adults free of cardiovascular disease [age 18-65 years, body mass index (BMI) $27-45 \mathrm{~kg} / \mathrm{m}^{2}$ and fasting blood glucose concentrations $<6.1 \mathrm{mmol} / \mathrm{L}]$ were recruited. Subjects using prescription 
medication or suffering from diseases or conditions that might influence the outcome of the study were excluded.

Of special interest were diseases that influenced body weight regulation (malabsorption, untreated hypo/hyperthyroidism, eating disorders, systemic use of steroids, etc.) and obesity-related cardiovascular risk factors (heart disease, systolic and diastolic blood pressures $\geq$

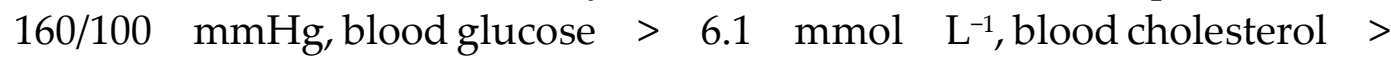

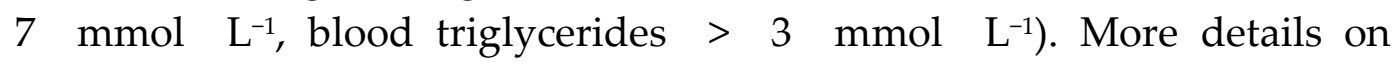
recruitment, inclusion and exclusion criteria, and study design are described elsewhere ${ }^{13}$. The Medical Ethical Committees of the respective countries approved the study protocol, which followed the Helsinki II regulations. The study was registered with ClinicalTrials.gov, number NCT00390637. Succinctly, the study consisted of two phases. In this report, we focus on the baseline characterization of the overweight/obese individuals as well as the weight-loss phase in which individuals with BMI $>27 \mathrm{~kg} / \mathrm{m}^{2}$ were assigned to lose weight following a LCD for 8 weeks. The LCD provided $3.3 \mathrm{MJ} / \mathrm{d}(800 \mathrm{kcal} / \mathrm{d}$ ) using Modifast products (Nutrition et Santé, France), with a macronutrient composition of $15-20 \%$ of total energy from fat, $35-40 \%$ from protein and $45-50 \%$ from carbohydrate. In addition, subjects could also eat up to $400 \mathrm{~g}$ of vegetables, providing a total of 3.3 to $4.2 \mathrm{MJ} / \mathrm{d}$ (800 to $1000 \mathrm{kcal} / \mathrm{d})$.

\subsection{Study Population}

We included 478 volunteers (303 women, 175 men) from whom plasma metabolomics (including acetate) were available before and after the LCD. Volunteers with BMI $>27 \mathrm{~kg} / \mathrm{m}^{2}$ were recruited in eight different research centers across Europe: Maastricht (the Netherlands), Copenhagen (Denmark), Cambridge (United Kingdom), Heraklion (Greece), Potsdam (Germany), Pamplona (Spain), Sofia (Bulgaria) and Prague (the Czech Republic). All subjects provided written informed consent before enrollment into the study.

\subsection{Anthropometry and blood sampling}

Anthropometry and blood samples were collected after fasting (10 hours) at baseline (clinical investigation day 1, CID1) and after weight loss at the end of the LCD period (CID2). In this report, we included the following parameters: anthropometry (body weight, BMI and fat-free mass (FFM)). FFM was assessed by multifrequency bioimpedance (QuadScan 4000, Bodystat, Douglas, United Kingdom) ${ }^{13}$. Additionally, glucose, free fatty acids (FFA) (automatic spectrophotometric enzymatic techniques) and insulin were measured from fasting samples ${ }^{13}$. Insulin concentrations were 
measured by a colorimetric assay (Ortho-Clinical Diagnostics, Johnson \& Johnson, Birkerød, Denmark) ${ }^{14}$.

Homeostatic Model Assessment of Insulin Resistance (HOMA-IR) and insulin sensitivity (Matsuda index) were calculated. HOMA-IR was calculated as follows: HOMA-IR $=$ [glucose $(\mathrm{mmol} / \mathrm{L}){ }^{*}$ insulin $(\mu \mathrm{U} / \mathrm{mL}) / 22.5]$, using fasting values ${ }^{15}$. Matsuda index was calculated as follows: (10,000/square root of (fasting glucose $\mathrm{x}$ fasting insulin) $\mathrm{x}$ (mean glucose $x$ mean insulin during Oral glucose tolerance test (OGTT))) ${ }^{16}$.

BMI was calculated by dividing the mass in $\mathrm{kg}$ by squared height. FFM was obtained from Bioimpedance analysis. Acetate, acetoacetate and 3-OH-butyrate were quantified in serum from nuclear magnetic resonance (NMR) spectra, as reported previously in detail ${ }^{17}$. Physical activity was measured before and after LCD by means of the Baecke questionnaire ${ }^{13}$.

\subsection{Statistical analysis}

Normality of data was assessed with the Kolmogorov-Smirnov procedure and histogram and variables that were not normally distributed were Ln-transformed. Repeated measures ANOVA with Bonferroni correction was conducted to investigate sex, time and sex-time effects as a result of LCD phase. A Linear mixed model (LMM) was used to determine the relationship between (changes in) acetate and (changes in) insulin sensitivity/resistance parameters at baseline and after LCD. In the first model besides (changes in) acetate and (changes in) fasting insulin, HOMAIR or Matsuda index, mean acetate were added. Subsequently, age (Model 2), (change in) weight (Model 3), and (change in) FFM (Model 4) were added as covariates. In all models, center was included as a random factor. 


\section{Results}

\subsection{Cross-sectional analysis at baseline}

Acetate showed significantly higher levels in men as compared to women at baseline $(1.35 \pm 1.39$ vs. $1.13 \pm 0.97 \mathrm{mmol} / \mathrm{L}$, respectively, See Table 1). There were no differences in BMI between males and females. Furthermore, fasting insulin, HOMA-IR, fasting glucose, body weight and FFM were higher in males, whilst the Matsuda index, FFA, and ketone bodies, were lower (See Table 1). There were no relationships between acetate and fasting insulin, HOMA-IR, and Matsuda Index in males as well as in females.

\subsection{Weight loss by LCD}

In response to the LCD, acetate levels increased in both men and women $(1.36 \pm 1.54$ to $1.41 \pm 1.25$ and $1.12 \pm 1.03$ to $1.18 \pm 1.04 \mathrm{mmol} / \mathrm{L}$, respectively, Table 1$)$, but did not differ between sexes $(P=0.672)$. Weight reduction increased insulin sensitivity as assessed by Matsuda $(P=0.002)$ and decreased HOMA-IR $(P=0.004)$ and fasting insulin $(P=0.001)$, with more pronounced improvements in males as compared to females. Additionally, FFA slightly increased as a result of weight loss with a more pronounced increase in males as compared to females (Table 1). Ketone bodies (acetoacetate and 3-OH-butyrate) showed an increase while acetate/3-OH-butyrate and acetate/acetoacetate ratios decreased after LCD, however sex-time interactions were not significant. Physical activity (sports and work indexes) did not change after LCD. Leisure activity differed (sex and time), however, sex-time interaction was not significant.

We observed a slight positive relationship between LCD-induced changes in acetate concentrations and changes in HOMA-IR (stdß 0.130, $P$ $=0.033$, Table 2$)$ and with changes in fasting insulin concentrations ( $\operatorname{std} \beta$ $0.119, P=0.051$, Table 3 ) in women, but not in men (std $\beta-0.072, P=0.310$ and std $\beta-0.066, P=0.359$, respectively; data not shown). Subsequently, the relationship between (changes in) acetate and HOMA-IR did not change after adjustment for age and changes in body weight as well as changes in FFM (Table 2). A similar result was seen for (changes in) fasting insulin (Table 3). The relationship between changes in acetate and Matsuda did not reach statistical significance (data not shown). 


\begin{tabular}{|c|c|c|c|c|c|c|c|}
\hline & \multicolumn{2}{|c|}{ Men } & \multicolumn{2}{|c|}{ Women } & \multirow[t]{2}{*}{ Sex } & \multirow[t]{2}{*}{ Time } & \multirow[t]{2}{*}{ Time $x$ sex } \\
\hline & $\begin{array}{l}\text { Baselin } \\
\mathrm{e}\end{array}$ & $\begin{array}{l}\text { After } \\
\text { LCD }\end{array}$ & Baseline & $\begin{array}{l}\text { After } \\
\text { LCD }\end{array}$ & & & \\
\hline $\mathrm{N}$ & 175 & 175 & 303 & 303 & & & \\
\hline Age (yr) & $42 \pm 6$ & $42 \pm 6$ & $41 \pm 6$ & $41 \pm 6$ & 0.000 & & 1 \\
\hline Acetate $(\mathrm{mmol} / \mathrm{L})$ & $\begin{array}{l}1.36 \pm \\
1.54\end{array}$ & $\begin{array}{l}1.41 \quad \pm \\
1.25\end{array}$ & $\begin{array}{l}1.12 \pm \\
1.03\end{array}$ & $\begin{array}{l}1.18 \quad \pm \\
1.04\end{array}$ & 0.000 & 0.016 & 0.672 \\
\hline HOMA index & $\begin{array}{l}3.86 \pm \\
2.35\end{array}$ & $\begin{array}{l}2.18 \quad \pm \\
2.23\end{array}$ & $2.93 \pm 3.0$ & $\begin{array}{l}2.00 \quad \pm \\
2.53\end{array}$ & 0.000 & 0.000 & 0.004 \\
\hline Matsuda index & $\begin{array}{l}4.10 \pm \\
2.50\end{array}$ & $\begin{array}{l}7.16 \quad \pm \\
3.8\end{array}$ & $\begin{array}{l}5.67 \quad \pm \\
3.50\end{array}$ & $\begin{array}{l}7.55 \quad \pm \\
3.70\end{array}$ & 0.000 & 0.000 & 0.002 \\
\hline Insulin $(\mu \mathrm{U} / \mathrm{mL})$ & $\begin{array}{l}13.8 \pm \\
7.6\end{array}$ & $8.1 \pm 5.3$ & $\begin{array}{l}11.14 \pm \\
11.14\end{array}$ & $\begin{array}{l}8.18 \quad \pm \\
9.32\end{array}$ & 0.000 & 0.000 & 0.001 \\
\hline Glucose $(\mathrm{mmol} / \mathrm{L})$ & $5.3 \pm 0.6$ & $5.0 \pm 0.5$ & $5.0 \pm 0.6$ & $4.8 \pm 0.3$ & 0.000 & 0.000 & 0.457 \\
\hline FFA (micromol/L) & $\begin{array}{l}528 \\
190\end{array}$ & $\begin{array}{l}634 \quad \pm \\
204\end{array}$ & $675 \pm 263$ & $746 \pm 213$ & 0.000 & 0.000 & 0.176 \\
\hline $\begin{array}{l}\text { Acetoacetate } \\
(\mathrm{mmol} / \mathrm{L})\end{array}$ & $\begin{array}{l}0.06 \quad \pm \\
0.04\end{array}$ & $\begin{array}{l}0.23 \quad \pm \\
0.30\end{array}$ & $\begin{array}{l}0.09 \quad \pm \\
0.07\end{array}$ & $\begin{array}{l}0.25 \quad \pm \\
0.30\end{array}$ & 0.000 & 0.000 & 0.376 \\
\hline $\begin{array}{l}\text { 3-OH-butyrate } \\
(\mathrm{mmol} / \mathrm{L})\end{array}$ & $\begin{array}{l}0.35 \quad \pm \\
0.30\end{array}$ & $\begin{array}{l}1.52 \quad \pm \\
2.00\end{array}$ & $\begin{array}{l}0.56 \quad \pm \\
0.52\end{array}$ & $\begin{array}{l}1.69 \pm \\
1.94\end{array}$ & 0.000 & 0.000 & 0.133 \\
\hline $\begin{array}{l}\text { Acetate/3-OH- } \\
\text { butyrate ratio }\end{array}$ & $5.80 \pm 8$ & $2.08 \pm 3$ & $\begin{array}{l}3.50 \quad \pm \\
4.90\end{array}$ & $\begin{array}{l}1.40 \quad \pm \\
2.04\end{array}$ & 0.000 & 0.000 & 0.415 \\
\hline $\begin{array}{l}\text { Acetate/Acetoaceta } \\
\text { te ratio }\end{array}$ & $26 \pm 29$ & $12 \pm 16$ & $18 \pm 21$ & $9 \pm 10$ & 0.000 & 0.000 & 0.787 \\
\hline Body weight (kg) & $\begin{array}{l}111.8 \pm \\
17.5\end{array}$ & $\begin{array}{l}99.0 \pm \\
15.9\end{array}$ & $\begin{array}{l}96.8 \quad \pm \\
16.5\end{array}$ & $86.6 \pm 15$ & 0.000 & 0.000 & 0.702 \\
\hline $\mathrm{BMI}\left(\mathrm{kg} / \mathrm{m}^{2}\right)$ & $35 \pm 4.8$ & $31 \pm 4.4$ & $35.2 \pm 5.1$ & $31.4 \pm 4.6$ & 0.259 & 0.000 & 0.657 \\
\hline Fat-free mass (kg) & $\begin{array}{l}73.4 \quad \pm \\
9.9\end{array}$ & $\begin{array}{ll}69.8 \quad \pm \\
8.7\end{array}$ & $53.4 \pm 8.1$ & $51 \pm 8$ & 0.000 & 0.000 & 0.990 \\
\hline \multicolumn{8}{|l|}{$\begin{array}{l}\text { Baecke } \\
\text { Questionnaire } \\
\text { scores }\end{array}$} \\
\hline Leisure index & $2.6 \pm 0.7$ & $2.8 \pm 0.6$ & $2.7 \pm 0.7$ & $3.0 \pm 0.6$ & 0.000 & 0.001 & 0.569 \\
\hline Sports index & $2.6 \pm 0.4$ & $2.6 \pm 0.4$ & $2.6 \pm 0.5$ & $2.7 \pm 0.4$ & 0.620 & 0.675 & 0.310 \\
\hline Work index & $2.7 \pm 0.4$ & $2.8 \pm 0.4$ & $2.7 \pm 0.4$ & $2.7 \pm 0.3$ & 0.087 & 0.670 & 0.980 \\
\hline
\end{tabular}

Repeated measures ANOVA. $1^{\text {st }} \mathbf{P}$-value reported corresponds to sex effect. $\mathbf{2}^{\text {nd }} \mathbf{P}$-value corresponds to time effect and $3^{\text {rd }} \mathbf{P}$-value corresponds to the sex-time effect. Data expressed as mean and standard deviation. Abbreviations: Low-calorie diet (LCD), Homeostatic Model Assessment of Insulin Resistance (HOMA-IR), BMI (Body Mass Index)

Table 2. Determinants of changes in HOMA-IR in participants during weight loss.

\begin{tabular}{|c|c|c|c|c|c|}
\hline \multirow[b]{2}{*}{ Model } & \multirow[b]{2}{*}{ Parameter } & \multicolumn{2}{|l|}{ Females } & \multicolumn{2}{|l|}{ Males } \\
\hline & & Std $B$, confidence interval & $\begin{array}{l}P \text { - } \\
\text { value }\end{array}$ & $\begin{array}{l}\text { Std B, confidence } \\
\text { interval }\end{array}$ & $\begin{array}{l}\text { P- } \\
\text { value }\end{array}$ \\
\hline 1 & $\begin{array}{l}\Delta \text {-Acetate } \\
\text { Mean } \\
\text { acetate }\end{array}$ & 0.111 (0.013 to 0.209$)$ & 0.027 & $\begin{array}{lll}-0.098 & (-0.224 \quad \text { to } \\
0.027) & & \end{array}$ & 0.123 \\
\hline 2 & $\begin{array}{l}\text { Model 1+ } \\
\text { Age }\end{array}$ & 0.125 (0.027 to 0.223$)$ & 0.013 & $\begin{array}{lll}-0.101 & (-0.227 \quad \text { to } \\
0.025) & & \end{array}$ & 0.115 \\
\hline 3 & $\begin{array}{l}\text { Model 2+ } \\
\Delta \text {-Weight }\end{array}$ & $0.120(0.022$ to 0.218$)$ & 0.017 & $\begin{array}{lll}-0.069 & (-0.189 & \text { to } \\
0.051) & & \end{array}$ & 0.259 \\
\hline 4 & $\begin{array}{l}\text { Model 3+ } \\
\Delta \text {-Fat-free } \\
\text { mass }\end{array}$ & 0.130 (0.010 to 0.249$)$ & 0.033 & $\begin{array}{lll}-0.072 & (-0.211 \quad \text { to } \\
0.068) & & \end{array}$ & 0.310 \\
\hline \multicolumn{6}{|c|}{$\begin{array}{l}\text { Linear Mixed model was adjusted for age, weight, and fat-free-mass. All models were adjusted for center as a random } \\
\text { factor (coefficients not shown). Acetate concentration (independent factor) and HOMA-IR as dependent factor. } \\
\text { Statistically significant P-values are in bold. Valid cases } \mathrm{N}=302 \text { females, } 175 \text { males. }\end{array}$} \\
\hline
\end{tabular}




\begin{tabular}{|c|c|c|c|c|c|}
\hline \multirow[b]{2}{*}{ Model } & \multirow[b]{2}{*}{ Parameter } & \multicolumn{2}{|l|}{ Females } & \multicolumn{2}{|l|}{ Males } \\
\hline & & Std $B$, confidence interval & $\begin{array}{l}\text { P- } \\
\text { value }\end{array}$ & $\begin{array}{l}\text { Std } B \text {, confidence } \\
\text { interval }\end{array}$ & $\begin{array}{l}P \text { - } \\
\text { value }\end{array}$ \\
\hline 1 & $\begin{array}{l}\Delta \text {-Acetate } \\
\text { Mean acetate }\end{array}$ & $0.132(0.034$ to 0.231$)$ & 0.009 & $\begin{array}{lll}-0.085 & (-0.212 & \text { to } \\
0.042) & & \end{array}$ & 0.190 \\
\hline 2 & $\begin{array}{l}\text { Model 1+ } \\
\text { Age }\end{array}$ & $0.140(0.042$ to 0.238$)$ & 0.005 & $\begin{array}{lll}-0.089 & (-0.217 \quad \text { to } \\
0.038) & & \end{array}$ & 0.168 \\
\hline 3 & $\begin{array}{l}\text { Model } 2+ \\
\Delta \text {-Weight }\end{array}$ & $0.132(0.035$ to 0.229$)$ & 0.008 & $\begin{array}{lll}-0.064 & (-0.188 & \text { to } \\
0.059) & & \end{array}$ & 0.306 \\
\hline 4 & $\begin{array}{l}\text { Model } 3+ \\
\Delta \text {-Fat-free } \\
\text { mass }\end{array}$ & $0.119(-0.001$ to 0.238$)$ & 0.051 & $\begin{array}{lll}-0.066 & (-0.207 & \text { to } \\
0.075) & & \end{array}$ & 0.359 \\
\hline \multicolumn{6}{|c|}{$\begin{array}{l}\text { Linear Mixed model was adjusted for age, weight, and fat-free-mass. All models were adjusted for center as a random } \\
\text { factor (coefficients not shown). Acetate concentration (independent factor) and HOMA-IR as dependent factor. } \\
\text { Statistically significant } P \text { values are in bold. Valid cases } N=295 \text { females, } 175 \text { males. }\end{array}$} \\
\hline
\end{tabular}

\section{Discussion}

In the present study we could not confirm previously reported positive associations between acetate and insulin sensitivity and metabolic health parameters in a cross-sectional analysis at baseline. LCD-induced weight loss increased insulin sensitivity more in men than in women. Surprisingly, we found a slight positive association between LCD-induced changes in acetate and changes in HOMA-IR and fasting insulin concentrations in overweight/obese women, but not in men, which was independent of age and changes in body composition.

As previously described, cross-sectional studies showed negative associations between acetate and fasting insulin in obese women 9 . Similarly, in morbidly obese subjects a negative association with HOMA-IR and a positive association with insulin sensitivity were observed ${ }^{10}$. Furthermore, acute distal colonic acetate infusions increased fat oxidation and improved metabolic profile in overweight/obese men ${ }^{6,7}$. Surprisingly, we observed a slight positive relationship between the LCD-induced change in circulating acetate and change in HOMA-IR and fasting insulin in females, but not in males. In line with these results on differential associations between males and females, a previous DiOGenes analysis by Stroeve et al. ${ }^{17}$, suggested a sex-specific modulatory role of baseline acetate in energy metabolism, since they reported a negative association with LCDinduced BMI change in morbidly obese females (stdß -0.23), but not in males. 
Furthermore, a previous analysis in the DiOGenes study showed that despite the fact that hepatic insulin resistance was lower in females as compared to males, there was a more pronounced worsening of blood lipid profile with the progression of hepatic insulin resistance in females, but not in males ${ }^{18}$.

In interpreting our results, it is important to note that acetate kinetics (acetate absorption in the colon, hepatic uptake and acetate clearance), and turnover (production/utilization) are intricate processes that are influenced by the interplay of gut microbial fermentation, lipogenesis, fat oxidation and ketogenesis ${ }^{19}$. With respect to insulinemic profile, hyperinsulinemia may affect acetate metabolism, possibly via an alteration in endogenous and exogenous acetate metabolism ${ }^{20}$. In addition, a caloric restriction may augment hepatic ketogenesis via an overproduction of acetyl-coA coming from increased lipid utilization ${ }^{21}$ and an increased ketogenesis may also elevate acetate production ${ }^{22}$. The exact mechanisms behind the sex-specific relationship of acetate and HOMA-IR may relate to sex hormones affecting processes such as microbial metabolism, hepatic lipid metabolism and ketogenesis. Notably, in our study most women were in the premenopausal state, however data on the hormone status was not available. Ketone bodies increased after the LCD with no differences between males and females. In addition, acetate/3-OH-butyrate and acetate/acetoacetate ratios decreased after LCD, which may suggest that the contribution of acetate from sources other than hepatic ketogenesis has probably not increased after LCD ${ }^{23}$.

Thus, the discrepancy of our findings on the relationship of acetate and insulin resistance as well as the sexual dimorphism in this relationship remains to be elucidated taking into account the complex acetate kinetics. Finally, other SCFA (propionate and butyrate) may be of importance for insulin sensitivity as we and other publications have reported 24-28. Unfortunately, these metabolites were not included in the NMR measurements and we focused on acetate, the most abundant SCFA in the colonic lumen and systemic circulation.

The major strength of our study is the availability of HOMA-IR and a 5-point Oral glucose tolerance test (including insulin concentrations for calculation of the Matsuda index) in a large and well-characterized cohort, which made additional adjustment for confounding factors feasible. A limitation of our study is the lack of information on acetate kinetics and colonic acetate that may contribute to circulating acetate concentrations. Of note, in the current study we measured plasma acetate concentrations using NMR methodology, which validation has been previously described $17,29,30$. Although, the absolute concentrations of acetate may differ as compared to previous studies measured with LC-MS or GC-MS 6-9, 25, 31, 32, high 
correlations have been reported between metabolites measured with both LC-MS and NMR ${ }^{33}$.

In conclusion, the results of this large pan-European study (478 volunteers) showed no relationship between acetate and markers of insulin sensitivity. Furthermore, a small positive association between LCDinduced changes in acetate and HOMA-IR and fasting insulin (explaining $1-2 \%$ of variance in HOMA-IR or fasting insulin) was shown in females and not in males. Future studies should aim to elucidate the underlying mechanisms and physiological significance. 


\section{References}

1. Canfora EE, Blaak EE. Acetate: a diet-derived key metabolite in energy metabolism: good or bad in context of obesity and glucose homeostasis? Curr Opin Clin Nutr Metab Care. 2017. Epub 2017/08/11. doi: 10.1097/mco.0000000000000408. PubMed PMID: 28795972.

2. den Besten G, Bleeker A, Gerding A, van Eunen K, Havinga R, van Dijk TH, et al. Short-Chain Fatty Acids Protect Against High-Fat DietInduced Obesity via a PPAR $\gamma$-Dependent Switch From Lipogenesis to Fat Oxidation. Diabetes. 2015;64(7):2398. doi: 10.2337/db14-1213.

3. Sahuri-Arisoylu M, Brody LP, Parkinson JR, Parkes H, Navaratnam $\mathrm{N}$, Miller $\mathrm{AD}$, et al. Reprogramming of hepatic fat accumulation and 'browning' of adipose tissue by the short-chain fatty acid acetate. International Journal of Obesity. 2016;40(6):955-63. doi: 10.1038/ijo.2016.23. 4. Perry RJ, Peng L, Barry NA, Cline GW, Zhang D, Cardone RL, et al. Acetate mediates a microbiome-brain- $\beta$-cell axis to promote metabolic syndrome. Nature. 2016;534(7606):213-7. doi: 10.1038/nature18309.

5. Freeland KR, Wolever TMS. Acute effects of intravenous and rectal acetate on glucagon-like peptide-1, peptide $Y Y$, ghrelin, adiponectin and tumour necrosis factor-alpha. The British journal of nutrition. 2010;103(3):460-6. doi: 10.1017/s0007114509991863. PubMed PMID: 19818198.

6. van der Beek CM, Canfora EE, Lenaerts K, Troost FJ, Damink S, Holst $\mathrm{JJ}$, et al. Distal, not proximal, colonic acetate infusions promote fat oxidation and improve metabolic markers in overweight/obese men. Clin Sci (Lond). 2016;130(22):2073-82. Epub 2016/07/22. doi: 10.1042/cs20160263. PubMed PMID: 27439969.

7. Canfora EE, van der Beek CM, Jocken JWE, Goossens GH, Holst JJ, Olde Damink SWM, et al. Colonic infusions of short-chain fatty acid mixtures promote energy metabolism in overweight/obese men: a randomized crossover trial. Sci Rep. 2017;7(1):2360. Epub 2017/05/26. doi: 10.1038/s41598-017-02546-x. PubMed PMID: 28539646; PubMed Central PMCID: PMCPMC5443817.

8. Neis EPJG, van Eijk HMH, Lenaerts K, Olde Damink SWM, Blaak $\mathrm{EE}$, Dejong $\mathrm{CHC}$, et al. Distal versus proximal intestinal short-chain fatty acid release in man. Gut. 2019;68(4):764. doi: 10.1136/gutjnl-2018-316161.

9. Layden BT, Yalamanchi SK, Wolever TM, Dunaif A, Lowe WL, Jr. Negative association of acetate with visceral adipose tissue and insulin levels. Diabetes, metabolic syndrome and obesity : targets and therapy. 2012;5:49-55. Epub 2012/02/28. doi: 10.2147/DMSO.S29244. PubMed PMID: 22419881. 
10. Moreno-Navarrete JM, Serino M, Blasco-Baque V, Azalbert V, Barton RH, Cardellini M, et al. Gut Microbiota Interacts with Markers of Adipose Tissue Browning, Insulin Action and Plasma Acetate in Morbid Obesity. Molecular Nutrition \& Food Research. 2018;62(3):1700721. doi: 10.1002/mnfr.201700721.

11. Robertson MD, Bickerton AS, Dennis AL, Vidal H, Frayn KN. Insulin-sensitizing effects of dietary resistant starch and effects on skeletal muscle and adipose tissue metabolism. Am J Clin Nutr. 2005;82(3):559-67. Epub 2005/09/13. doi: 10.1093/ajcn.82.3.559. PubMed PMID: 16155268.

12. Most J, Goossens GH, Reijnders D, Canfora EE, Penders J, Blaak EE. Gut microbiota composition strongly correlates to peripheral insulin sensitivity in obese men but not in women. Benef Microbes. 2017;8(4):55762. Epub 2017/06/18. doi: 10.3920/bm2016.0189. PubMed PMID: 28618864.

13. Larsen TM, Dalskov S, van Baak M, Jebb S, Kafatos A, Pfeiffer A, et al. The Diet, Obesity and Genes (Diogenes) Dietary Study in eight European countries - a comprehensive design for long-term intervention. Obes Rev. 2010;11(1):76-91. Epub 2009/05/28. doi: 10.1111/j.1467-789X.2009.00603.x. PubMed PMID: 19470086.

14. Goyenechea E, Holst C, van Baak MA, Saris WH, Jebb S, Kafatos A, et al. Effects of different protein content and glycaemic index of ad libitum diets on diabetes risk factors in overweight adults: the DIOGenes multicentre, randomized, dietary intervention trial. Diabetes Metab Res Rev. 2011;27(7):705-16. Epub 2011/05/19. doi: 10.1002/dmrr.1218. PubMed PMID: 21591241.

15. Matthews DR, Hosker JP, Rudenski AS, Naylor BA, Treacher DF, Turner RC. Homeostasis model assessment: insulin resistance and beta-cell function from fasting plasma glucose and insulin concentrations in man. Diabetologia. 1985;28(7):412-9. Epub 1985/07/01. doi: 10.1007/bf00280883. PubMed PMID: 3899825.

16. Matsuda M, DeFronzo RA. Insulin sensitivity indices obtained from oral glucose tolerance testing: comparison with the euglycemic insulin clamp. Diabetes Care. 1999;22(9):1462. doi: 10.2337/diacare.22.9.1462.

17. Stroeve JHM, Saccenti E, Bouwman J, Dane A, Strassburg K, Vervoort J, et al. Weight loss predictability by plasma metabolic signatures in adults with obesity and morbid obesity of the DiOGenes study. Obesity. 2016;24(2):379-88. doi: 10.1002/oby.21361.

18. van der Kolk BW, Vogelzangs $\mathrm{N}$, Jocken JWE, Valsesia A, Hankemeier T, Astrup A, et al. Plasma lipid profiling of tissue-specific insulin resistance in human obesity. International Journal of Obesity. 2019;43(5):989-98. doi: 10.1038/s41366-018-0189-8.

19. Piloquet H, Ferchaud-Roucher V, Duengler F, Zair Y, Maugere P, Krempf M. Insulin effects on acetate metabolism. Am J Physiol Endocrinol 
Metab. 2003;285(3):E561-5. Epub 2003/05/30. doi: 10.1152/ajpendo.00042.2003. PubMed PMID: 12773304.

20. Fernandes J, Vogt J, Wolever TMS. Kinetic model of acetate metabolism in healthy and hyperinsulinaemic humans. European Journal of Clinical Nutrition. 2014;68(9):1067-71. doi: 10.1038/ejcn.2014.136.

21. Knowles SE, Jarrett IG, Filsell OH, Ballard FJ. Production and utilization of acetate in mammals. The Biochemical journal. 1974;142(2):40111. doi: 10.1042/bj1420401. PubMed PMID: 4441381.

22. Leighton F, Bergseth S, Rørtveit T, Christiansen EN, Bremer J. Free acetate production by rat hepatocytes during peroxisomal fatty acid and dicarboxylic acid oxidation. The Journal of biological chemistry. 1989;264(18):10347-50. PubMed PMID: 2732225.

23. SEUFERT CD, MEWES W, SOELING HD. Effect of long-term starvation on acetate and ketone body metabolism in obese patients. European Journal of Clinical Investigation. 1984;14(2):163-70. doi: 10.1111/j.1365-2362.1984.tb02107.x.

24. Sanna S, van Zuydam NR, Mahajan A, Kurilshikov A, Vich Vila A, Võsa $U$, et al. Causal relationships among the gut microbiome, short-chain fatty acids and metabolic diseases. Nature Genetics. 2019;51(4):600-5. doi: 10.1038/s41588-019-0350-x.

25. Müller M, Hernández MAG, Goossens GH, Reijnders D, Holst JJ, Jocken JWE, et al. Circulating but not faecal short-chain fatty acids are related to insulin sensitivity, lipolysis and GLP-1 concentrations in humans. Scientific Reports. 2019;9(1):12515. doi: 10.1038/s41598-019-48775-0.

26. Todesco T, Rao AV, Bosello O, Jenkins DJ. Propionate lowers blood glucose and alters lipid metabolism in healthy subjects. The American Journal of Clinical Nutrition. 1991;54(5):860-5. doi: 10.1093/ajcn/54.5.860.

27. Wolever TM, Brighenti F, Royall D, Jenkins AL, Jenkins DJ. Effect of rectal infusion of short chain fatty acids in human subjects. Am J Gastroenterol. 1989;84(9):1027-33. Epub 1989/09/01. PubMed PMID: 2773895.

28. Gao Z, Yin J, Zhang J, Ward RE, Martin RJ, Lefevre M, et al. Butyrate Improves Insulin Sensitivity and Increases Energy Expenditure in Mice. Diabetes. 2009;58(7):1509. doi: 10.2337/db08-1637.

29. Emwas A-H, Roy R, McKay RT, Tenori L, Saccenti E, Gowda GAN, et al. NMR Spectroscopy for Metabolomics Research. Metabolites. 2019;9(7):123. doi: 10.3390/metabo9070123. PubMed PMID: 31252628.

30. Dona AC, Kyriakides M, Scott F, Shephard EA, Varshavi D, Veselkov $\mathrm{K}$, et al. A guide to the identification of metabolites in NMR-based metabonomics/metabolomics experiments. Comput Struct Biotechnol J. 2016;14:135-53. Epub 2016/04/19. doi: 10.1016/j.csbj.2016.02.005. PubMed PMID: 27087910; PubMed Central PMCID: PMCPMC4821453. 
31. Canfora EE, van der Beek CM, Hermes GDA, Goossens GH, Jocken JWE, Holst JJ, et al. Supplementation of Diet With Galacto-oligosaccharides Increases Bifidobacteria, but Not Insulin Sensitivity, in Obese Prediabetic Individuals. Gastroenterology. 2017;153(1):87-97.e3. Epub 2017/04/12. doi: 10.1053/j.gastro.2017.03.051. PubMed PMID: 28396144.

32. Gathungu RM, Kautz R, Kristal BS, Bird SS, Vouros P. The integration of LC-MS and NMR for the analysis of low molecular weight trace analytes in complex matrices. Mass Spectrometry Reviews. 2020;39(12):35-54. doi: 10.1002/mas.21575.

33. Lanza IR, Zhang S, Ward LE, Karakelides H, Raftery D, Nair KS. Quantitative Metabolomics by 1H-NMR and LC-MS/MS Confirms Altered Metabolic Pathways in Diabetes. PLoS One. 2010;5(5):e10538. doi: 10.1371/journal.pone.0010538. 


\section{CHAPTER 4}

\section{Circulating but not fecal short-chain fatty acids are related to insulin sensitivity, lipolysis and GLP-1 concentrations in humans}

Running title: Circulating and fecal SCFA in metabolic health

Mattea Müller;; Manuel A. González Hernández;; Gijs H. Goossens, Dorien Reijnders, Jens J. Holst; Johan W.E. Jocken, Hans van Eijk, Emanuel E. Canfora; and Ellen E. Blaak

\#These authors contributed equally to the work

Scientific Reports 9, 12515 (2019) 


\begin{abstract}
Microbial-derived short-chain fatty acids (SCFA) acetate, propionate and butyrate may provide a link between gut microbiota and whole-body insulin sensitivity (IS). In this cross-sectional study (160 participants, $66.3 \%$ male, BMI: $19.2-41.0 \mathrm{~kg} / \mathrm{m}^{2}$, normal or impaired glucose metabolism), associations between SCFA (fecal and fasting circulating) and circulating metabolites, substrate oxidation and IS were investigated. In a subgroup $(\mathrm{n}=93)$, IS was determined using a hyperinsulinemic-euglycemic clamp. Data were analyzed using multiple linear regression analysis adjusted for sex, age and BMI. Fasting circulating acetate, propionate and butyrate concentrations were positively associated with fasting GLP-1 levels. Additionally, circulating SCFA were negatively related to whole-body lipolysis (glycerol), triacylglycerols and free fatty acids levels (standardized (std) $\beta$ adjusted (adj) -0.190, $P=0.023$; std $\beta$ adj $-0.202, P=0.010$; std $\beta$ adj $0.306, P=0.001$, respectively). Circulating acetate and propionate were, respectively, negatively and positively correlated with IS (M-value: std $\beta$ adj $-0.294, \mathrm{P}<0.001$; std $\beta$ adj $0.161, \mathrm{P}=0.033$, respectively). We show that circulating rather than fecal SCFA were associated with GLP-1 concentrations, whole-body lipolysis and peripheral IS in humans. Therefore, circulating SCFA are more directly linked to metabolic heath, which indicates the need to measure circulating SCFA in human prebiotic/probiotic intervention studies as a biomarker/mediator of effects on host metabolism.
\end{abstract}




\section{Introduction}

In obesity and type 2 diabetes mellitus (T2DM), alterations in the gut microbiota composition and functionality may contribute to disease aetiology. The gut microbiota ferments indigestible carbohydrates (i.e. dietary fibres) and major end-products thereof are the short- chain fatty acids (SCFA) acetate, propionate and butyrate ${ }^{1}$. Acetate, propionate and butyrate are present in the colon in a ratio of approximately 3:1:1, respectively ${ }^{2,3}$. Most butyrate is utilized by colonocytes as energy source ${ }^{4}$. Via the portal vein, SCFA reach the liver where acetate and propionate are metabolized and partly oxidised or used as substrate in gluconeogenesis and lipogenesis ${ }^{5}$. Consequently, a small proportion of microbial-derived SCFA enters the peripheral circulation whereby acetate reaches the highest concentrations compared to propionate and butyrate ${ }^{6,7}$. SCFA are ligands to G-protein coupled receptors (GPR) 41 and 43, which are expressed on intestinal, adipose, skeletal muscle, liver and pancreatic tissues 8-10, indicating their important role in the crosstalk between the gut and peripheral tissues. Several rodent studies showed that oral, intravenous and colonic infusion of SCFA as well as microbial-derived SCFA beneficially affect the functioning and metabolism of the aforementioned tissues and consequently improve insulin sensitivity, substrate metabolism and body weight regulation ${ }^{11}$. In humans, distal colonic acetate infusions increased fasting fat oxidation, energy expenditure, and PYY secretion whilst whole-body lipolysis was decreased ${ }^{12,13}$. Other acute studies show decreased circulating free fatty acid (FFA) concentration after rectal SCFA infusion in healthy participants ${ }^{14,15}$. In addition, 24 weeks of $10 \mathrm{~g} /$ day inulin propionate ester protected against body weight gain as compared to inulin only in overweight individuals ${ }^{16}$. Potential mechanisms may include a SCFA-induced inhibition of energy intake possibly mediated via the stimulation of glucagon-like peptide 1 (GLP-1) and peptide YY (PYY) secretion, increased intestinal gluconeogenesis, increased skeletal muscle fat oxidation and improved lipid buffering capacity of adipose tissue ${ }^{11}$.

However, increased microbial-derived acetate formation has been associated with increased body weight gain and insulin resistance in dietinduced obese rats ${ }^{17}$. Additionally, increased fecal SCFA have been reported in overweight and obese compared to lean participants $2,3,8,18,19$, yet it is difficult to interpret the latter data since fecal SCFA reflect the net result of colonic production and absorption ${ }^{20,21}$. Even though fecal SCFA are commonly used as an indicator of microbial fermentation, fecal SCFA may not accurately reflect in vivo colonic fermentation since approximately $95 \%$ of colonic SCFA are absorbed and only the remaining 5\% are excreted in feces ${ }^{22-25}$. 
To obtain more information on the validity of fecal SCFA as biomarker for metabolic health effects, the associations between fecal and circulating SCFA concentrations and parameters of metabolic health were studied in a relatively large cohort of 160 participants with a wide range of body mass indices (BMI) and glucometabolic status. Using multiple regression analysis, we analysed the relationship between fecal and fasting circulating SCFA with fasting glucose, insulin, circulating lipids (free fatty acids (FFA), triacylglycerols (TAG), glycerol), insulin resistance index (homeostasis model assessment of insulin resistance (HOMA-IR)), gut hormone concentrations (PYY, GLP-1), fasting substrate utilization and inflammation markers including lipopolysaccharide-binding protein (LBP), tumour necrosis factor alpha (TNF- $\alpha$ ), interleukin 6 (IL-6) and interleukin 8 (IL-8). We further investigated the relationship between fecal and fasting circulating SCFA profiles and peripheral insulin sensitivity index (M-value) as measured via the gold standard hyperinsulinaemic-euglycemic clamp technique in a subset of individuals.

\section{Methods}

\section{Study participants}

This cross-sectional analysis included 160 Caucasian men and women aged 20-70 years with a BMI between 19.2 and $41.0 \mathrm{~kg} / \mathrm{m}^{2}$ from the general population in the vicinity of Maastricht, The Netherlands during August 2013 and December 2016. Individuals had normoglycemia, impaired fasting glucose (IFG, $\geq 5.6 \mathrm{mmol} / \mathrm{L}$ ) and/or impaired glucose tolerance (IGT, 2 hour plasma glucose of $7.8-11 \mathrm{mmol} / \mathrm{L}$ after $75 \mathrm{~g}$ oral glucose challenge) according to the diagnostic criteria of the American Diabetes Association, $2010{ }^{26}$. Eligibility of the participants was assessed via a general health questionnaire, medical history and anthropometry during an initial screening visit. Exclusion criteria were as follows: use of antibiotics, prebiotics, or probiotics 3 months before the study, diagnosis of T2DM, gastrointestinal or cardiovascular diseases, abdominal surgery, participants with life expectancy shorter than 5 years and participants following a hypocaloric diet. Participants did not use $\beta$-blockers, lipid- or glucose-lowering drugs, anti-oxidants, or chronic corticosteroids. All protocols were reviewed and approved by the local Medical Ethical Committee (MUMC+) and conducted in accordance with the Declaration of Helsinki (revised version, October 2008, Seoul, South Korea). Written informed consent was obtained from all participants. 


\section{Study Design}

This cross-sectional analysis included metabolic parameters as well as fecal and fasting circulating SCFA concentrations of previously performed intervention studies $12,13,27-29$. In the present study, we collated and analyzed study data at baseline and thus prior to the respective interventions.

In all studies, sample collection was performed after an overnight fast, and measurements were conducted according to the same standard operating procedures. Two days prior to the baseline investigation day, participants were asked to refrain from intense physical activity and alcohol consumption, and to collect a fecal sample. In the evening before the investigation day, the participants consumed a standardized low-fiber meal.

\section{Used data sets}

The data set included baseline data from the following intervention human in vivo studies. These include an intervention study in prediabetic, overweight-obese individuals on the effect of antibiotics on insulin sensitivity (Clinical trial No. NCT02241421) ${ }^{28}$, an intervention study in prediabetic, overweight-obese individuals on the effect of dietary fiber (galacto-oligosaccharides) on insulin sensitivity (Clinical trial No. NCT02271776) ${ }^{27}$, an intervention study in normoglycemic, normal to overweight individuals on the effect of dietary fibers on gastrointestinal transit (Clinical trial No. NCT02491125 ) ${ }^{29}$, and lastly two acute studies investigating the effect of different mixtures of SCFA in normoglycemic, overweight to obese individuals on human substrate and energy metabolism (Clinical trial No. NCT01826162 12, Clinical trial No. NCT01983046 $\left.{ }^{13}\right)$.

\section{Baseline investigation day}

After an overnight fast ( $>10 \mathrm{~h})$, participants came to the laboratory by car or public transport. Anthropometry was measured including height, weight and waist to hip ratio. After inserting a cannula into the antecubital vein, blood samples were taken to measure plasma metabolites, hormones and inflammatory markers in the fasted state. After the blood sampling, participants were in a resting, half-supine position and fasting substrate oxidation was measured for $30 \mathrm{~min}$ using an open circuit ventilated hood system (Omnical, MUMC+, Maastricht, the Netherlands). Fat and carbohydrate oxidation were calculated according to the equations of Weir and Frayn ${ }^{30,31}$, assuming that protein oxidation accounted for $15 \%$ of total energy expenditure. 


\section{Hyperinsulinaemic-euglycaemic clamp}

Peripheral insulin sensitivity was determined in a subgroup of overweight/obese, prediabetic individuals via hyperinsulinaemiceuglycemic clamps as previously described ${ }^{27,28}$. In short, a cannula was inserted into an antecubital vein for infusion of glucose and insulin. To measure blood glucose, a second cannula was inserted into a superficial dorsal hand vein, which was arterialized by placing the hand into a hotbox $\left(\sim 50^{\circ} \mathrm{C}\right)$.

A priming dose of insulin infusion (Actrapid, Novo Nordisk, Gentofte, Denmark) was administered during the first ten $\min (\mathrm{t} 0-\mathrm{t} 10 \mathrm{~min})$ and insulin infusion was thereafter continued at $40 \mathrm{mU} / \mathrm{m}^{2} / \mathrm{min}$ for $2 \mathrm{~h}(\mathrm{t} 10$ - t120 min). By variable infusion of a $20 \%$ glucose solution, plasma concentrations were maintained at $5.0 \mathrm{mmol} / \mathrm{L}$. Peripheral insulin sensitivity (M-value, $\mathrm{mg}^{*}\left(\mathrm{~kg}^{*} \mathrm{~min}\right)^{-1}$ was calculated from the mean glucose infusion rate during the steady-state of the clamp (last 30min, stable blood glucose concentration at $5.0 \mathrm{mmol} / \mathrm{L}$ ) ${ }^{32}$. A high $\mathrm{M}$-value represents high insulin sensitivity (i.e., more glucose needs to be infused to maintain euglycemia during insulin infusion).

\section{Analysis of fecal and circulating SCFA}

Fecal samples were collected at home and stored in the subjects' freezer at $-20{ }^{\circ} \mathrm{C}$ maximum of two days before the baseline investigation day, transported on dry ice, and stored on arrival at the university at $-80^{\circ} \mathrm{C}$. Fecal acetate, propionate, and butyrate were measured by gas chromatography-mass spectrometry (Dr. Stein and Colleague Medical Laboratory, Mönchengladbach, Germany) as previously described ${ }^{33}$. Plasma sample preparation for circulating SCFA analysis were performed as reported previously ${ }^{34}$. In short, deproteinization was performed by mixing 1 part plasma (v/v) with 2 parts methanol acidified with $1.5 \mathrm{mmol} / \mathrm{l}$ hydrochloric acid. Subsequently, samples were vortex-mixed vigorously and immediately centrifuged at 50000xg in a model Biofuge Stratos (Hereaus, Dijkstra Vereenigde, Lelystad, the Netherlands) for $15 \mathrm{~min}$ at $4^{\circ} \mathrm{C}$. Then, $100 \mu \mathrm{l}$ aliquots of the clear plasma supernatant were transferred into glass micro-insert vials and stored in the Combi-Pal until analysis. Samples were calibrated against external standards. The reversed phase separation was performed on a X-select ODS $2.5 \mu \mathrm{m}$ column (150mm x 2.1mm I.D., Waters, Breda, the Netherlands), mounted in a Mistral Spark column oven (Separations, H.I. Ambacht, the Netherlands), set to $45^{\circ} \mathrm{C}$. Samples were completely separated from other components into the individual SCFA in a $25 \mathrm{~min}$. gradient cycle between an aqueous $1 \mathrm{mmol} / \mathrm{l}$ solution of sulfuric acid and ethanol. 
Post-column, the solvent $\mathrm{pH}$ was enhanced to about 9 , by mixing with $150 \mathrm{mmol} / \mathrm{l}$ ammonia in ethanol to maximize negative ionization. Samples were processed using a Combi-Pal sample processor (Interscience, Breda, the Netherlands) with Peltier chilled sample storage compartments set to $10^{\circ} \mathrm{C}$. The system was equipped with a $50 \mu \mathrm{l}$ sample loop. Separated SCFA were detected using a model LTQ XL linear ion trap mass spectrometer (Thermo Fisher Scientific, Breda, the Netherlands), equipped with an ion-max electrospray probe. The MS was operated in MS-MS full scan negative mode."

\section{Blood collection and biochemical analysis}

Blood was collected in pre-chilled EDTA tubes $(0.2 \mathrm{~mol} / \mathrm{L}$ EDTA; Sigma, Dorset, UK) for SCFA, insulin, glucose, FFA, TAG, free glycerol, LBP, GLP-1, TNF- $\alpha$, IL- 6 and IL-8 analyses during fasting conditions. For GLP-1 and PYY analysis, $20 \mu \mathrm{l}$ of dipeptidyl peptidase-IV inhibitor (Milipore Merck, Billerica, MA, USA) was added to EDTA and Aprotinin (Becton Dickinson, Eysins, Switzerland) tubes, respectively. Samples were centrifuged at $3500 \mathrm{~g}, 4{ }^{\circ} \mathrm{C}$ for 10 minutes; plasma was aliquoted and directly snap-frozen in liquid nitrogen and stored at $-80^{\circ} \mathrm{C}$ until analysis. Plasma glucose concentrations were determined using commercially available reagent kit (Glucose Hexokinase CP, Horiba ABX Pentra, Montpellier, France) involving a two-step enzymatic reaction with hexokinase followed by Glucose-6-phosphate-dehydrogenase resulting in D-gluconate-6-phosphate. The colorimetric reaction was measured using an automated spectrophotometer (ABX Pentra 400 autoanalyzer, Horiba ABX Pentra). Plasma FFA concentrations were measured using a commercially available kit (NEFA-HR(2) assay, Wako, Sopachem BV, Ochten, the Netherlands) with a two-step enzymatic reaction involving acylation of Coenzyme(Co) A followed by acyl-CoA oxidase resulting in the production of hydrogen peroxide as substrate that in the presence of peroxidase yields a blue purple pigment, measured with a colorimetric reaction measured using an automated spectrophotometer (ABX Pentra 400 autonalyzer, Horiba ABX Pentra). Plasma TAG were determined using a commercially available kit (Triglycerides CP, Horiba ABX Pentra) based on enzymatic reactions involving lipoprotein lipase, glycerolkinase and glycerol-3-phosphate oxidase resulting in the production of hydrogen peroxide as substrate of a colorimetric reaction measured using the automated spectrophotometer (ABX Pentra 400 autonalyzer, Horiba ABX Pentra). Plasma glycerol was measured after precipitation with an enzymatic assay (Enzytec TM Glycerol, Roche Biopharm, Basel, Switzerland) involving phosphorylation of glycerol to L-glycerol-3phosphate by glycerokinase and the colorimetric reaction is measured 
using an automated spectrophotometer (Cobas Fara, Roche Diagnostics, Basel, Switzerland). Plasma insulin was determined with a commercially available radioimmunoassay (RIA) kit (HI-14K Human Insulin specific RIA, Millipore Merck) according to the manufacture's protocol. Plasma IL 6, IL- 8 and TNF- $\alpha$ were determined with an commercialy available enzymelinked immunosorbent assay (ELISA) kit (Human Proinflammatory II 4Plex Ultra-Sensitive kit, Meso Scale Diagnostics, MD, USA). Plasma samples were assayed for total GLP-1 immunoreactivity using an antiserum that reacts equally with intact GLP-1 and the primary (Nterminally truncated) metabolite as previously described ${ }^{35}$.

PYY concentrations were determined using a commercially available radio-immunoassay (RIA) kits (Human PYY (3-36) Specific RIA, Millipore Merck). Plasma LBP was measured as previously described ${ }^{36}$. In short, plates (Greiner Mocrolon 600 high binding; Sigma Aldrich, St. Louis, MO) were coated with polyclonal anti-human LBP antibodies. Diluted plasma samples (1:5000) and a standard dilution series with recombinant LBP were added to the plate. Detection occurred with a biotinylated polyclonal rabbit anti-human LBP IgG, followed by peroxidase-conjugated streptavidin and substrate. The detection limit for the LBP assay was $200 \mathrm{pg} / \mathrm{ml}$.

\section{Statistical analysis}

Normality of data was assessed with the Gaussian distribution and Kolmogorov-Smirnov procedure, and ln or Z-score transformation was used if assumption of normality was not met. HOMA-IR was calculated as previously described ${ }^{37}$. In case of missing data, the participant was excluded from the analysis. Multicollinearity was checked using variance inflation factor index $<10$. First, we used simple linear regression to investigate the associations between fecal and circulating concentrations of acetate, propionate and butyrate (as dependent variables) and metabolic parameters (as independent variables) i.e. insulin sensitivity (M-value), insulin resistance (HOMA-IR), circulating glucose, insulin, circulating lipids (TAG, FFA and glycerol), circulating inflammatory markers (IL-6, IL8, TNF- $\alpha$ and LBP) and fasting substrate oxidation. Subsequently, we used multiple linear regression to test whether the associations between fecal and circulating SCFA and the aforementioned metabolic parameters were independent of the covariates sex, age and BMI. All data were analysed using SPSS 22.0 (IBM, Armok, U.S.) with significance set at $\mathrm{P}<0.05$. 


\section{Results}

Mean age of the participants was $49.6 \pm 14.7$ years and $66.3 \%$ of participants were male with a mean BMI of $29.8 \pm 4.4 \mathrm{~kg} / \mathrm{m}^{2}$, a mean fasting glucose of $5.6 \pm 0.6 \mathrm{mmol} / \mathrm{L}$ and a mean HOMA-IR of $3.7 \pm 1.5$ (Table 1). In the subgroup, peripheral insulin sensitivity (M-value) was measured in 93 overweight to obese, prediabetic men $(n=72)$ and women $(n=21)$ with mean age of $59.0 \pm 7.1$ years and a mean BMI of $31.8 \pm 3.1 \mathrm{~kg} / \mathrm{m}^{2}$, respectively.

Table 1 Characteristics of participants

\begin{tabular}{|c|c|c|}
\hline Characteristic & Mean \pm SD & Range \\
\hline Male (n) /Female (n) & $106 / 54$ & \\
\hline Age $(y)$ & $49.6 \pm 14.7$ & $20-69$ \\
\hline Body Weight (kg) & $90.8 \pm 15.7$ & $52.8-125.9$ \\
\hline BMI $\left(\mathrm{kg} / \mathrm{m}^{2}\right)$ & $29.8 \pm 4.4$ & $19.2-41.0$ \\
\hline Waist:Hip ratio & $0.93 \pm 0.13$ & $0.64-1.22$ \\
\hline HOMA-IR & $3.7 \pm 1.5$ & $1.0-9.0$ \\
\hline Fasting plasma insulin (mU/L) & $14.9 \pm 9.4$ & $2.4-82.7$ \\
\hline Fasting plasma glucose (mmol/L) & $5.6 \pm 0.6$ & $3.1-7.5$ \\
\hline Fasting plasma free fatty acids $(\mu \mathrm{mol} / \mathrm{L})$ & $672 \pm 243$ & $140.3-1620$ \\
\hline Fasting plasma triacylglycerol $(\mu \mathrm{mol} / \mathrm{L})$ & $1178 \pm 613$ & $311.0-3944$ \\
\hline Fasting free glycerol $(\mu \mathrm{mol} / \mathrm{L})$ & $102.1 \pm 46$ & $27.1-372.1$ \\
\hline Plasma acetate $(\mu \mathrm{mol} / \mathrm{L})$ & $65.2 \pm 64.7$ & $2.8-429.4$ \\
\hline Plasma propionate $(\mu \mathrm{mol} / \mathrm{L})$ & $2.0 \pm 1.6$ & $0.06-12.0$ \\
\hline $\begin{array}{l}\text { Plasma butyrate }(\mu \mathrm{mol} / \mathrm{L}) \\
\text { Fecal acetate }(\mu \mathrm{mol} / \mathrm{g}) \\
\text { Fecal propionate }(\mu \mathrm{mol} / \mathrm{g}) \\
\text { Fecal butyrate }(\mu \mathrm{mol} / \mathrm{g})\end{array}$ & $\begin{array}{l}1.7 \pm 1.8 \\
44.2 \pm 16.1 \\
13.0 \pm 7.9 \\
10.9 \pm 5.9\end{array}$ & $\begin{array}{l}0.07-6.7 \\
6.80-102.8 \\
2.53-71.2 \\
0.00-33.8\end{array}$ \\
\hline \multicolumn{3}{|c|}{ Subgroup hyperinsulinaemic-euglycaemic clamp } \\
\hline Male (n) /Female (n) & $72 / 21$ & \\
\hline BMI $\left(\mathrm{kg} / \mathrm{m}^{2}\right)$ & $31.8 \pm 3.1$ & 26.1- 41 \\
\hline Age (y) & $59.0 \pm 7.1$ & $72-69$ \\
\hline Fasting glucose (mmol/L) & $6.0 \pm 0.5$ & $5.0-7.5$ \\
\hline 2h glucose $(\mathrm{mmol} / \mathrm{L})$ & $7.2 \pm 1.8$ & $3.3-11.2$ \\
\hline M-value $\left(\mathrm{mg}^{*} \mathrm{~kg}^{-1 *} \mathrm{~min}^{-1}\right)$ & $3.6 \pm 1.5$ & $1.7-8.3$ \\
\hline \multicolumn{3}{|c|}{$\begin{array}{l}\text { BMI body mass index, HOMA-IR Homeostasis model assessment of insulin resistance, M- } \\
\text { value mean glucose infusion rate at steady state during insulinemic- euglycemic clamps }\end{array}$} \\
\hline
\end{tabular}




\section{Associations between fecal and circulating SCFA concentrations}

Fecal acetate and butyrate were not associated to their respective circulating concentrations, while fecal propionate was positively associated with circulating propionate (standardized (std) std $\beta=0.262, \mathrm{P}=0.002$ ).

\section{Circulating, but not fecal, SCFA are associated with BMI}

Fecal acetate was positively associated with BMI $($ std $\beta=0.245, P=$ $0.004)$, however, after adjustment for age and sex, the association was not significant anymore (std $\beta$ adj $=0.092, \mathrm{P}=0.214$ ). Fecal propionate and butyrate were not significantly associated with BMI with or without adjustment for age and sex (fecal butyrate std $\beta=0.162, P=0.055$, fecal propionate std $\beta=0.023, \mathrm{P}=0.746)$. In contrast, circulating butyrate and propionate were significantly inversely related to BMI, also after adjustment for age and sex (circulating butyrate std $\beta=-0.599 \mathrm{P}<0.001$, circulating propionate std $\beta=-0.290, \mathrm{P}<0.001$, Supplementary Figure $1 \mathrm{~K}$, L). Circulating acetate was negatively associated with BMI (std $\beta=-0.285$, $\mathrm{P}<0.001)$, which was not significant anymore after adjustment for age and $\operatorname{sex}(\operatorname{std} \beta=-0.115, P=0.108)$.

\section{Fecal SCFA were not related to metabolic parameters}

None of fecal SCFA were significantly associated with fasting GLP1, PYY, FFA, TAG, glycerol, glucose, insulin concentrations, HOMA-IR, inflammatory markers or fasting substrate oxidation either with or without adjustment for age, sex and BMI. In the subgroup analysis fecal SCFA were not associated with peripheral insulin sensitivity (Table 2). 
Table 2 Simple and multiple linear regression coefficients between fecal SCFA and metabolic parameters in fasting state

\begin{tabular}{|c|c|c|c|c|c|c|c|c|c|c|c|c|}
\hline \multirow[b]{2}{*}{ Metabolites } & \multicolumn{4}{|c|}{ Fecal acetate } & \multicolumn{4}{|c|}{ Fecal propionate } & \multicolumn{4}{|c|}{ Fecal butyrate } \\
\hline & $\operatorname{std} \beta$ & $P$ & $\begin{array}{l}\text { std } \\
\text { adj }\end{array}$ & $P$ & $\operatorname{std} \beta$ & $P$ & $\begin{array}{l}\text { std } \\
\text { adj }\end{array}$ & $P$ & $\operatorname{std} \beta$ & $P$ & $\begin{array}{l}\text { std } \\
\text { adj }\end{array}$ & $P$ \\
\hline FFA, $\mu \mathrm{mol} / \mathrm{L}$ & $\begin{array}{l}0.052 \pm \\
0.090\end{array}$ & .569 & $\begin{array}{l}-0.048 \pm \\
0.082\end{array}$ & .558 & $\begin{array}{l}-0.098 \pm \\
0.089\end{array}$ & .276 & $\begin{array}{l}-0.092 \pm \\
0.080\end{array}$ & .249 & $\begin{array}{l}0.001 \pm \\
0.090\end{array}$ & .991 & $\begin{array}{l}-0.030 \pm \\
0.081\end{array}$ & .707 \\
\hline $\mathrm{TAG}, \mu \mathrm{mol} / \mathrm{L}$ & $\begin{array}{c}0.058 \pm \\
0.090\end{array}$ & .519 & $\begin{array}{l}-0.057 \pm \\
0.084\end{array}$ & .498 & $\begin{array}{l}0.129 \pm \\
0.089\end{array}$ & .151 & $\begin{array}{l}0.037 \pm \\
0.081\end{array}$ & .647 & $\begin{array}{l}0.094 \pm \\
0.090\end{array}$ & .296 & $\begin{array}{l}-0.007 \pm \\
0.082\end{array}$ & .931 \\
\hline $\begin{array}{l}\text { Glycerol, } \\
\mu \mathrm{mol} / \mathrm{L}\end{array}$ & $\begin{array}{l}-0.047 \pm \\
0.090\end{array}$ & .603 & $\begin{array}{l}-0.152 \pm \\
0.086\end{array}$ & .079 & $\begin{array}{l}-0.019 \pm \\
0.090\end{array}$ & .836 & $\begin{array}{l}-0.022 \pm \\
0.084\end{array}$ & .797 & $\begin{array}{l}-0.185 \pm \\
0.145\end{array}$ & .205 & $\begin{array}{l}-0.160 \pm \\
0.084\end{array}$ & .059 \\
\hline \multicolumn{13}{|l|}{ Hormones } \\
\hline Insulin, $\mathrm{mU} / \mathrm{L}$ & $\begin{array}{l}0.275 \pm \\
0.082\end{array}$ & .001 & $\begin{array}{l}0.122 \pm \\
0.071\end{array}$ & .088 & $\begin{array}{l}0.185 \pm \\
0.084\end{array}$ & .029 & $\begin{array}{l}0.114 \pm \\
0.069\end{array}$ & .101 & $\begin{array}{l}0.207 \pm \\
0.084\end{array}$ & .015 & $\begin{array}{l}0.102 \pm \\
0.070\end{array}$ & .146 \\
\hline $\begin{array}{l}\text { GLP-1, } \\
\text { pmol/L }\end{array}$ & $\begin{array}{l}-0.081 \pm \\
0.092\end{array}$ & .379 & $\begin{array}{l}0.114 \pm \\
0.074\end{array}$ & .125 & $\begin{array}{l}0.017 \pm \\
0.092\end{array}$ & .852 & $\begin{array}{l}0.140 \pm \\
0.071\end{array}$ & .053 & $\begin{array}{l}-0.037 \pm \\
0.092\end{array}$ & .692 & $\begin{array}{l}0.116 \pm \\
0.072\end{array}$ & .113 \\
\hline PYY, pmol/L & $\begin{array}{l}-0.194 \pm \\
0.106\end{array}$ & .070 & $\begin{array}{l}-0.166 \pm \\
0.112\end{array}$ & .140 & $\begin{array}{l}-0.093 \pm \\
0.107\end{array}$ & .386 & $\begin{array}{l}-0.087 \pm \\
0.110\end{array}$ & .428 & $\begin{array}{l}-0.156 \pm \\
0.106\end{array}$ & .146 & $\begin{array}{l}-0.142 \pm \\
0.110\end{array}$ & .199 \\
\hline \multicolumn{13}{|c|}{ Insulin sensitivity } \\
\hline $\begin{array}{l}\text { M-value, } \\
\mathrm{mg} / \mathrm{kg} / \mathrm{min}\end{array}$ & $\begin{array}{l}-0.071 \pm \\
0.106\end{array}$ & .502 & $\begin{array}{l}-0.035 \pm \\
0.075\end{array}$ & .640 & $\begin{array}{l}-0.029 \pm \\
0.105\end{array}$ & .783 & $\begin{array}{l}-0.050 \pm \\
0.073\end{array}$ & .490 & $\begin{array}{l}-0.100 \pm \\
0.105\end{array}$ & .345 & $\begin{array}{l}-0.111 \pm \\
0.073\end{array}$ & .130 \\
\hline HOMA-IR & $\begin{array}{c}0.267 \pm \\
0.084\end{array}$ & .002 & $\begin{array}{l}0.080 \pm \\
0.068\end{array}$ & .244 & $\begin{array}{l}0.193 \pm \\
0.085\end{array}$ & .025 & $\begin{array}{l}0.091 \pm \\
0.066\end{array}$ & .169 & $\begin{array}{l}0.214 \pm \\
0.085\end{array}$ & .013 & $\begin{array}{l}0.076 \pm \\
0.067\end{array}$ & .254 \\
\hline $\begin{array}{l}\text { Glucose, } \\
\text { mmol/L }\end{array}$ & $\begin{array}{l}0.174 \pm \\
0.084\end{array}$ & .040 & $\begin{array}{l}-0.029 \pm \\
0.067\end{array}$ & .661 & $\begin{array}{l}0.112 \pm \\
0.084\end{array}$ & .187 & $\begin{array}{l}0.006 \pm \\
0.065\end{array}$ & .925 & $\begin{array}{l}0.086 \pm \\
0.085\end{array}$ & .310 & $\begin{array}{l}-0.066 \pm \\
0.066\end{array}$ & .315 \\
\hline \multicolumn{13}{|c|}{ Inflammatory markers } \\
\hline LBP, pg/ml & $\begin{array}{c}0.152 \pm \\
0.091\end{array}$ & .095 & $\begin{array}{l}0.033 \pm \\
0.086\end{array}$ & .699 & $\begin{array}{l}0.024 \pm \\
0.091\end{array}$ & .789 & $\begin{array}{l}-0.010 \pm \\
0.084\end{array}$ & .909 & $\begin{array}{l}0.155 \pm \\
0.090\end{array}$ & .088 & $\begin{array}{l}0.092 \pm \\
0.084\end{array}$ & .274 \\
\hline $\mathrm{IL}-6, \mathrm{pg} / \mathrm{ml}$ & $\begin{array}{l}0.164 \pm \\
0.085\end{array}$ & .055 & $\begin{array}{l}-0.010 \pm \\
0.071\end{array}$ & .892 & $\begin{array}{l}0.012 \pm \\
0.085\end{array}$ & .887 & $\begin{array}{l}-0.088 \pm \\
0.069\end{array}$ & .204 & $\begin{array}{l}0.039 \pm \\
0.085\end{array}$ & .651 & $\begin{array}{l}-0.094 \pm \\
0.069\end{array}$ & .178 \\
\hline IL-8, pg/ml & $\begin{array}{c}0.157 \pm \\
0.085\end{array}$ & .067 & $\begin{array}{l}0.106 \pm \\
0.088\end{array}$ & .231 & $\begin{array}{l}0.164 \pm \\
0.084\end{array}$ & .053 & $\begin{array}{l}0.118 \pm \\
0.085\end{array}$ & .170 & $\begin{array}{l}0.115 \pm \\
0.085\end{array}$ & .179 & $\begin{array}{l}0.061 \pm \\
0.086\end{array}$ & .479 \\
\hline $\mathrm{TNF}-\alpha, \mathrm{pg} / \mathrm{ml}$ & $\begin{array}{c}0.196 \pm \\
0.084\end{array}$ & .021 & $\begin{array}{l}0.070 \pm \\
0.078\end{array}$ & .170 & $\begin{array}{l}0.063 \pm \\
0.085\end{array}$ & .459 & $\begin{array}{l}-0.046 \pm \\
0.076\end{array}$ & .548 & $\begin{array}{l}0.156 \pm \\
0.084\end{array}$ & .067 & $\begin{array}{l}0.039 \pm \\
0.076\end{array}$ & .612 \\
\hline \multicolumn{13}{|c|}{ Substrate Oxidation } \\
\hline Fat, E\% & $\begin{array}{l}0.036 \\
0.092\end{array}$ & .691 & $\begin{array}{l}0.001 \pm \\
0.095\end{array}$ & .996 & $\begin{array}{l}-0.002 \pm \\
0.091\end{array}$ & .982 & $\begin{array}{l}0.004 \pm \\
0.093\end{array}$ & .970 & $\begin{array}{l}0.013 \pm \\
0.092\end{array}$ & .885 & $\begin{array}{l}0.004 \pm \\
0.094\end{array}$ & .969 \\
\hline CHO, E \% & $\begin{array}{l}-0.092 \pm \\
0.094\end{array}$ & .329 & $\begin{array}{l}-0.084 \pm \\
0.098\end{array}$ & .397 & $\begin{array}{l}-0.034 \pm \\
0.094\end{array}$ & .716 & $\begin{array}{l}-0.042 \pm \\
0.096\end{array}$ & .666 & $\begin{array}{l}-0.110 \pm \\
0.094\end{array}$ & .243 & $\begin{array}{l}-0.116 \pm \\
0.096\end{array}$ & .233 \\
\hline
\end{tabular}

$\beta$, standardized $\beta$ coefficient + standard error of coefficient of fecal acetate, propionate and butyrate as dependent variable in a simple regression and multiple regression analysis adjusted for age, sex and BMI. M-value (n=93), PYY (n=107). Adj, adjusted, std standardized, E\%, percentage of energy expenditure, FFA, free fatty acids, TAG, triglycerides, GLP-1, glucagon-like peptide 1, PYY, peptide YY, HOMA-IR, homeostatic model assessment of insulin resistance, LBP, lipopolysaccharide binding protein, IL-6, interleukin 6, IL-8, interleukin 8, TNF $\alpha$, tumour necrosis factor alpha. 
Table 3 Simple and multiple linear regression coefficients between circulating SCFA and metabolic parameters in fasting state

\begin{tabular}{|c|c|c|c|c|c|c|c|c|c|c|c|c|}
\hline \multirow[b]{2}{*}{ Metabolites } & \multicolumn{4}{|c|}{ Circulating acetate } & \multicolumn{4}{|c|}{ Circulating propionate } & \multicolumn{4}{|c|}{ Circulating butyrate } \\
\hline & $\operatorname{std} \beta$ & $P$ & $\begin{array}{ll}\text { std } & \beta \\
\text { adj } & \end{array}$ & & $\operatorname{std} \beta$ & $P$ & std $\beta$ adj & & $\operatorname{std} \beta$ & $P$ & $\begin{array}{ll}\text { std } & \beta \\
\text { adj } & \end{array}$ & $P$ \\
\hline FFA, $\mu \mathrm{mol} / \mathrm{L}$ & $\begin{array}{l}-0.032 \pm \\
0.084\end{array}$ & .701 & $\begin{array}{l}0.003 \pm \\
0.080\end{array}$ & .974 & $\begin{array}{l}-0.302 \pm \\
0.079\end{array}$ & .000 & $\begin{array}{l}-0.127 \pm \\
0.078\end{array}$ & .103 & $\begin{array}{l}-0.381 \pm \\
0.077\end{array}$ & $<.001$ & $\begin{array}{l}-0.306 \\
\pm 0.090\end{array}$ & .001 \\
\hline $\mathrm{TAG}, \mu \mathrm{mol} / \mathrm{L}$ & $\begin{array}{l}-0.249 \pm \\
0.081\end{array}$ & .003 & $\begin{array}{l}-0.077 \pm \\
0.081\end{array}$ & .340 & $\begin{array}{l}-0.225 \pm \\
0.081\end{array}$ & .006 & $\begin{array}{l}-0.202 \pm \\
0.078\end{array}$ & .010 & $\begin{array}{l}-0.356 \pm \\
0.078\end{array}$ & $<.001$ & $\begin{array}{l}-0.137 \\
\pm 0.094\end{array}$ & .150 \\
\hline $\begin{array}{l}\text { Glycerol, } \\
\mu \mathrm{mol} / \mathrm{L}\end{array}$ & $\begin{array}{l}-0.204 \pm \\
0.082\end{array}$ & .014 & $\begin{array}{l}-0.190 \pm \\
0.083\end{array}$ & .023 & $\begin{array}{l}-0.025 \pm \\
0.083\end{array}$ & .768 & $\begin{array}{l}0.155 \pm \\
0.081\end{array}$ & .059 & $\begin{array}{l}-0.286 \pm \\
0.080\end{array}$ & $<.001$ & $\begin{array}{l}-0.173 \\
\pm 0.098\end{array}$ & .079 \\
\hline \multicolumn{13}{|l|}{ Hormones } \\
\hline $\begin{array}{l}\text { Insulin, } \\
\mathrm{mU} / \mathrm{L}\end{array}$ & $\begin{array}{l}-0.187 \pm \\
0.079\end{array}$ & .019 & $\begin{array}{l}0.003 \pm \\
0.070\end{array}$ & .968 & $\begin{array}{l}-0.234 \pm \\
0.078\end{array}$ & .003 & $\begin{array}{l}-0.066 \pm \\
0.068\end{array}$ & .336 & $\begin{array}{l}-0.406 \pm \\
0.073\end{array}$ & $<.001$ & $\begin{array}{l}-0.040 \\
\pm 0.082\end{array}$ & .625 \\
\hline $\begin{array}{l}\text { GLP-1, } \\
\mathrm{pmol} / \mathrm{L}\end{array}$ & $\begin{array}{l}0.402 \pm \\
0.078\end{array}$ & .000 & $\begin{array}{l}0.187 \pm \\
0.070\end{array}$ & .009 & $\begin{array}{l}0.318 \pm \\
0.080\end{array}$ & .000 & $\begin{array}{l}0.218 \pm \\
0.068\end{array}$ & .002 & $\begin{array}{l}0.574 \pm \\
0.069\end{array}$ & $<.001$ & $\begin{array}{l}0.274 \pm \\
0.081\end{array}$ & .001 \\
\hline PYY, pmol/L & $\begin{array}{l}0.125 \pm \\
0.097\end{array}$ & .201 & $\begin{array}{l}0.107 \pm \\
0.107\end{array}$ & .317 & $\begin{array}{l}0.080 \pm \\
0.097\end{array}$ & .413 & $\begin{array}{l}0.018 \pm \\
0.105\end{array}$ & .868 & $\begin{array}{l}0.182 \pm \\
0.096\end{array}$ & .060 & $\begin{array}{l}0.142 \pm \\
0.126\end{array}$ & .263 \\
\hline \multicolumn{13}{|c|}{ Insulin sensitivity } \\
\hline $\begin{array}{l}\text { M-value, } \\
\mathrm{mg} / \mathrm{kg} / \mathrm{min}\end{array}$ & $\begin{array}{l}-0.224 \pm \\
0.102\end{array}$ & .031 & $\begin{array}{l}-0.294 \pm \\
0.071\end{array}$ & $<.001$ & $\begin{array}{l}0.327 \pm \\
0.099\end{array}$ & .001 & $\begin{array}{l}0.161 \pm \\
0.074\end{array}$ & .033 & $\begin{array}{l}0.164 \pm \\
0.103\end{array}$ & .113 & $\begin{array}{l}-0.066 \\
\pm 0.091\end{array}$ & .469 \\
\hline HOMA-IR & $\begin{array}{l}-0.247 \pm \\
0.079\end{array}$ & .002 & $\begin{array}{l}-0.009 \pm \\
0.067\end{array}$ & .892 & $\begin{array}{l}-0.160 \pm \\
0.080\end{array}$ & .047 & $\begin{array}{l}0.007 \pm \\
0.065\end{array}$ & .914 & $\begin{array}{l}-0.461 \pm \\
0.072\end{array}$ & $<.001$ & $\begin{array}{l}-0.054 \\
\pm 0.078\end{array}$ & .494 \\
\hline $\begin{array}{l}\text { Glucose, } \\
\text { mmol/L }\end{array}$ & $\begin{array}{l}-0.228 \pm \\
0.078\end{array}$ & .004 & $\begin{array}{l}0.005 \pm \\
0.066\end{array}$ & .939 & $\begin{array}{l}-0.177 \pm \\
0.078\end{array}$ & .025 & $\begin{array}{l}-0.044 \pm \\
0.064\end{array}$ & .499 & $\begin{array}{l}-0.506 \pm \\
0.068\end{array}$ & $<.001$ & $\begin{array}{l}-0.200 \\
\pm 0.075\end{array}$ & $<.001$ \\
\hline \multicolumn{13}{|c|}{ Inflammatory markers } \\
\hline LBP, pg/ml & $\begin{array}{l}-0.075 \pm \\
0.088\end{array}$ & .399 & $\begin{array}{l}0.038 \pm \\
0.087\end{array}$ & .667 & $\begin{array}{l}-0.262 \pm \\
0.085\end{array}$ & .003 & $\begin{array}{l}-0.117 \pm \\
0.085\end{array}$ & .168 & $\begin{array}{l}-0.330 \pm \\
0.083\end{array}$ & $<.001$ & $\begin{array}{l}-0.116 \\
\pm 0.102\end{array}$ & .254 \\
\hline IL-6, pg/ml & $\begin{array}{l}-0.298 \pm \\
0.077\end{array}$ & .000 & $\begin{array}{l}-0.098 \pm \\
0.070\end{array}$ & .163 & $\begin{array}{l}-0.177 \pm \\
0.079\end{array}$ & .027 & $\begin{array}{l}-0.027 \pm \\
0.053\end{array}$ & .690 & $\begin{array}{l}-0.406 \pm \\
0.073\end{array}$ & .000 & $\begin{array}{l}-0.021 \\
\pm 0.082\end{array}$ & .803 \\
\hline IL-8, pg/ml & $\begin{array}{l}-0.079 \pm \\
0.080\end{array}$ & .324 & $\begin{array}{l}0.024 \pm \\
0.086\end{array}$ & .778 & $\begin{array}{l}0.126 \pm \\
0.079\end{array}$ & .113 & $\begin{array}{l}0.164 \pm \\
0.084\end{array}$ & .051 & $\begin{array}{l}-0.023 \pm \\
0.080\end{array}$ & .775 & $\begin{array}{l}0.185 \pm \\
0.100\end{array}$ & .067 \\
\hline $\begin{array}{l}\text { TNF- } \alpha, \\
\mathrm{pg} / \mathrm{ml}\end{array}$ & $\begin{array}{l}-0.296 \pm \\
0.076\end{array}$ & .000 & $\begin{array}{l}-0.105 \pm \\
0.076\end{array}$ & .168 & $\begin{array}{l}-0.095 \pm \\
0.079\end{array}$ & .232 & $\begin{array}{l}-0.048 \pm \\
0.075\end{array}$ & .523 & $\begin{array}{l}-0.330 \pm \\
0.075\end{array}$ & $<.001$ & $\begin{array}{l}-0.047 \\
\pm 0.089\end{array}$ & .598 \\
\hline \multicolumn{13}{|c|}{ Substrate Oxidation } \\
\hline Fat, E\% & $\begin{array}{l}-0.156 \pm \\
0.084\end{array}$ & .065 & $\begin{array}{l}-0.021 \pm \\
0.086\end{array}$ & .812 & $\begin{array}{l}-0.117 \pm \\
0.084\end{array}$ & .167 & $\begin{array}{l}-0.052 \pm \\
0.085\end{array}$ & .543 & $\begin{array}{l}-0.317 \pm \\
0.080\end{array}$ & $<.001$ & $\begin{array}{l}-0.138 \\
\pm 0.101\end{array}$ & .172 \\
\hline CHO, E \% & $\begin{array}{l}0.074 \pm \\
0.087\end{array}$ & .396 & $\begin{array}{l}0.159 \pm \\
0.094\end{array}$ & .094 & $\begin{array}{l}-0.092 \pm \\
0.087\end{array}$ & .291 & $\begin{array}{l}-0.167 \pm \\
0.092\end{array}$ & .072 & $\begin{array}{l}0.002 \pm \\
0.087\end{array}$ & .986 & $\begin{array}{l}0.047 \pm \\
0.111\end{array}$ & .672 \\
\hline
\end{tabular}

$\beta$, standardized $\beta$ coefficient + standard error of coefficient of fasting circulating acetate, propionate and butyrate as dependent variable in a simple regression and multiple regression analysis adjusted for age, sex and BMI. M-value (n=93), PYY (n=107). Adj, adjusted, std standardized, E\%, percentage of energy expenditure, FFA, free fatty acids, TAG, triglycerides, GLP-1, glucagon-like peptide 1, PYY, peptide YY, HOMA-IR, homeostatic model assessment of insulin resistance, LBP, lipopolysaccharide binding protein, IL-6, interleukin 6 , IL-8, interleukin 8 , TNF $\alpha$, tumour necrosis factor alpha.

\section{Fasting, circulating SCFA were related to fasting GLP-1, lipid metabolites and insulin sensitivity}

All three circulating SCFA were positively associated with fasting GLP-1 concentrations (Table 3). Additionally, circulating acetate, propionate and butyrate were negatively associated with fasting glycerol, TAG and FFA, respectively. Also, circulating butyrate was negatively associated with fasting glucose. These relationships remained significant after adjustment for age, sex and BMI (Table 3, Supplementary Figure 1). Circulating SCFA were not associated with fasting PYY, LBP, IL-6, IL-8 and TNF- $\alpha$. Furthermore, circulating SCFA were not related to fat and carbohydrate oxidation, expressed as percentage of energy expenditure. In the subgroup analysis of overweight/obese, prediabetic individuals, 
peripheral insulin sensitivity was measured using the M-value derived from the hyperinsulinaemic- euglycaemic clamp technique.

We found that circulating acetate was negatively associated with peripheral insulin sensitivity (M-value) whereas circulating propionate was positively related to peripheral insulin sensitivity (Table 3, Supplementary Figure S1). The relationships between circulating SCFA and insulin sensitivity remained significant after adjustment for age, sex and BMI.

\section{Discussion}

We investigated the relationship between fecal and fasting circulating SCFA with fasting plasma metabolites, gut hormones, substrate metabolism and inflammatory markers in a cohort with a wide range of BMI and glucometabolic status. This study shows that only circulating but not fecal SCFA concentrations were related to fasting plasma glucose, FFA, TAG and glycerol, GLP-1 and insulin sensitivity, also after adjustment for age, sex and BMI. Contrary to previous human studies, fecal SCFA were not related to BMI, whereas circulating butyrate and propionate were inversely associated with BMI. Circulating plasma propionate seems to be the most reflective of its respective fecal concentrations, whilst fecal acetate and butyrate were not related to their respective circulating concentrations. In line, previous literature reports that SCFA flux into the circulation and uptake in peripheral tissues rather than microbial SCFA production per se is of importance for metabolic health ${ }^{38-40}$. Our data emphasize the need to measure circulating SCFA in human prebiotic/probiotic intervention studies as a biomarker/mediator of effects on host metabolism.

To our knowledge, this is the first study providing evidence that fasting circulating SCFA are positively associated with fasting plasma GLP1 in humans. High colonic SCFA production is linked to increased GLP-1 and PYY secretion through binding of SCFA to GPR41/43 on the enteroendocrine L-cell ${ }^{41}$. Further, a one year dietary fiber intervention (wheat bran, 24g/d) increased circulating SCFA concentrations accompanied by increased levels of GLP-1 concentrations in hyperinsulinemic participants ${ }^{42}$. Yet, there is little known about the contribution of circulating SCFA to GLP-1 secretion during the fasted state. Circulating SCFA may stimulate GLP-1 secretion from the visceral, basolateral side of enteroendocrine L-cells as observed in isolated rat colons 43. Besides enteroendocrine L-cells, pancreatic $\alpha$-cells have been suggested to contribute to systemic GLP-1 concentrations in the fasted state ${ }^{44,45}$, but whether circulating SCFA act as stimuli for GLP-1 secretion warrants further investigation. 
In contrast to GLP-1, we did not find an association between circulating and fecal SCFA with fasting PYY. This is in contrast to human and in vitro studies reporting a stimulatory effect of SCFA on PYY secretion $12,46,47$, however to what extent SCFA and/or dietary fibres contribute to fasting PYY secretion remains to be investigated. Although the mechanisms still remain to be elucidated, the present data indicate that despite being the net result of production, uptake and tissue utilization, circulating SCFA are more directly linked to metabolic health as compared to fecal SCFA.

In our study population, only circulating, but not fecal SCFA were associated with fasting plasma metabolites. Circulating acetate was negatively associated with fasting free glycerol, an indicator of whole-body lipolysis. This is consistent with in vitro and human in vivo studies reporting that acetate has an antilipolytic effect ${ }^{13,48-50}$. This may be beneficial for metabolic health in the long term, since partial inhibition of adipose tissue lipolysis may reduce systemic lipid spillover thereby attenuating ectopic lipid accumulation ${ }^{51}$. Furthermore, circulating propionate was negatively associated with fasting TAG, which might be explained by the activating effect of propionate on lipoprotein lipase (LPL) in adipose tissue leading to increased TAG extraction as shown in vitro ${ }^{52}$. Furthermore, circulating butyrate was negatively associated with fasting FFA concentrations. In vitro data about the lipolytic effect of butyrate are contradictive showing proand antilipolytic effects of butyrate in white adipose tissue models ${ }^{50,53}$. Thus, circulating SCFA may be negatively related to systemic glycerol or FFA and/or TAG suggesting that increased circulating SCFA may reduce systemic lipid overflow with a potential beneficial effect on ectopic lipid accumulation and insulin sensitivity.

Nevertheless, with respect to markers of insulin sensitivity, neither fasting circulating nor fecal SCFA were related to fasting insulin or HOMAIR in the total study population. Yet, fasting circulating butyrate, but not acetate and propionate, was negatively associated with fasting glucose. This is consistent with rodent studies showing that butyrate administration may have glucose lowering effects and may improve insulin sensitivity in the postprandial state ${ }^{54,55}$. In obesity, insulin resistance and T2DM, the abundance of butyrate-producing bacteria is reduced, which may explain to some extent the inverse association between circulating butyrate and fasting glucose in our study ${ }^{56-58}$. 
In the subgroup analysis including prediabetic individuals with obesity, circulating acetate was negatively associated with peripheral insulin sensitivity. This is in contrast with previous rodent studies reporting a beneficial role of acetate on insulin sensitivity ${ }^{47}$ and with two small-scale human cross-sectional studies including obese women or morbidly obese individuals reported either none or a positive association of circulating acetate and insulin sensitivity measured via hyperinsulinemic-euglycemic clamp, respectively 59, 60. Additionally, when acetate is administered colonically, overweight participants showed increases in fasting fat oxidation, energy expenditure, and PYY secretion ${ }^{12,13}$, reflective of positive effects on metabolic health. Interestingly, a kinetic study showed that intravenously infused acetate remains longer in the circulation in individuals with T2DM suggesting a disturbed acetate tissue uptake and metabolism in the context of metabolic disorders ${ }^{47}$. Furthermore, exogenous and endogenous acetate production but not colonic acetate absorption differed between hyperinsulinemic and normoinsulinemic individuals after rectal infusion of sodium-acetate $49,61,62$. Thus, our findings may reflect an altered endogenous acetate metabolism rather than an altered microbial-derived acetate production in metabolically compromised individuals. In contrast to fasting circulating acetate, fasting circulating propionate was positively associated with clamp-derived insulin sensitivity. Propionate has been reported to stimulate glucose uptake in 3T3-L1 adipocytes and C2C12 skeletal muscle cells in vitro and improve insulin sensitivity (HOMA-IR) in mice fed a high fat diet 63,64 . Possible mechanisms include an increase in peripheral glucose uptake via increased GPR41 stimulation, suppression of hepatic de novo lipogenesis and increase formation of beneficial odd chain fatty acids in the liver ${ }^{65}$.

The main limitation of our study is the cross-sectional design, which limits causal suppositions. Further, we cannot account for endogenous SCFA production, splanchnic and liver extraction or tissue utilization in this study ${ }^{66,67}$. Secondly, measures of GLP-1 and SCFA in the postprandial state would have been valuable. However, the study's major strength is the availability of fecal and fasting circulating SCFA in combination with metabolic markers in a relatively large cohort with a broad range of BMI and metabolic health status. This enabled us to investigate the relationship between fecal and fasting circulating SCFA concentrations with markers of lipid and energy metabolism as well as insulin sensitivity measured by the gold standard hyperinsulinemic-euglycemic clamp. 
For the first time, we confirmed that fasting circulating but not fecal SCFA were related to whole-body lipolysis, fasting GLP-1 and insulin sensitivity in the fasted state. Furthermore, our study calls for urgently needed mechanistic studies in humans concerning the relationship between SCFA, GLP-1 secretion and lipid metabolism.

In conclusion, our data show that circulating but not fecal SCFA are linked to circulating GLP-1 concentrations, whole-body lipolysis and peripheral insulin sensitivity in humans. Of note, this highlights that circulating SCFA are more directly linked to metabolic heath parameters. Therefore, our data indicate the need to measure circulating SCFA as a biomarker/mediator of effects on host metabolism in future human prebiotic/probiotic intervention studies. This may provide interesting leads for future research, which should aim to modulate the SCFA availability in the systemic circulation and its impact on peripheral tissue function. 


\section{References}

1. Macfarlane GT, Macfarlane S. Bacteria, Colonic Fermentation, and Gastrointestinal Health. Journal of AOAC International. 2012;95(1):50-60. doi: 10.5740/jaoacint.SGE_Macfarlane.

2. Schwiertz A, Taras D, Schafer K, Beijer S, Bos NA, Donus C, et al. Microbiota and SCFA in lean and overweight healthy subjects. Obesity (Silver Spring). 2010;18(1):190-5. Epub 2009/06/06. doi: 10.1038/oby.2009.167. PubMed PMID: 19498350.

3. Fernandes J, Su W, Rahat-Rozenbloom S, Wolever TMS, Comelli EM. Adiposity, gut microbiota and faecal short chain fatty acids are linked in adult humans. Nutrition \& Diabetes. 2014;4(6):e121. doi: 10.1038/nutd.2014.23. PubMed PMID: PMC4079931.

4. Roediger WE. Role of anaerobic bacteria in the metabolic welfare of the colonic mucosa in man. Gut. 1980;21(9):793-8. PubMed PMID: 7429343. 5. Boets E, Gomand SV, Deroover L, Preston T, Vermeulen K, De Preter $\mathrm{V}$, et al. Systemic availability and metabolism of colonic-derived shortchain fatty acids in healthy subjects: a stable isotope study. J Physiol. 2017;595(2):541-55. doi: 10.1113/JP272613. PubMed PMID: PMC5233652.

6. Kroger J, Zietemann V, Enzenbach C, Weikert C, Jansen EH, Doring $\mathrm{F}$, et al. Erythrocyte membrane phospholipid fatty acids, desaturase activity, and dietary fatty acids in relation to risk of type 2 diabetes in the European Prospective Investigation into Cancer and Nutrition (EPIC)Potsdam Study. Am J Clin Nutr. 2011;93(1):127-42. Epub 2010/10/29. doi: 10.3945/ajcn.110.005447. PubMed PMID: 20980488.

7. Bloemen JG, Venema K, van de Poll MC, Olde Damink SW, Buurman WA, Dejong $\mathrm{CH}$. Short chain fatty acids exchange across the gut and liver in humans measured at surgery. Clinical Nutrition. 2009;28(6):657-61. doi: 10.1016/j.clnu.2009.05.011.

8. Tang C, Ahmed K, Gille A, Lu S, Grone H-J, Tunaru S, et al. Loss of FFA2 and FFA3 increases insulin secretion and improves glucose tolerance in type 2 diabetes. Nat Med. 2015;21(2):173-7. doi: 10.1038/nm.3779.

9. Koh A, De Vadder F, Kovatcheva-Datchary P, Bäckhed F. From Dietary Fiber to Host Physiology: Short-Chain Fatty Acids as Key Bacterial Metabolites. Cell. 2016;165(6):1332-45. doi: 10.1016/j.cell.2016.05.041.

10. Priyadarshini M, Villa SR, Fuller M, Wicksteed B, Mackay CR, Alquier T, et al. An Acetate-Specific GPCR, FFAR2, Regulates Insulin Secretion. Molecular Endocrinology. 2015;29(7):1055-66. doi: 10.1210/me.2015-1007.

11. Canfora EE, Jocken JW, Blaak EE. Short-chain fatty acids in control of body weight and insulin sensitivity. Nat Rev Endocrinol. 2015;11(10):57791. doi: 10.1038/nrendo.2015.128. 
12. van der Beek CM, Canfora EE, Lenaerts K, Troost FJ, Damink SW, Holst JJ, et al. Distal, not proximal, colonic acetate infusions promote fat oxidation and improve metabolic markers in overweight/obese men. Clin Sci (Lond). 2016;130(22):2073-82. Epub 2016/07/22. doi: 10.1042/cs20160263. PubMed PMID: 27439969.

13. Canfora EE, van der Beek CM, Jocken JWE, Goossens GH, Holst JJ, Olde Damink SWM, et al. Colonic infusions of short-chain fatty acid mixtures promote energy metabolism in overweight/obese men: a randomized crossover trial. Scientific Reports. 2017;7(1):2360. doi: 10.1038/s41598-017-02546-x.

14. Wolever TM, Spadafora P, Eshuis H. Interaction between colonic acetate and propionate in humans. Am J Clin Nutr. 1991;53(3):681-7. Epub 1991/03/01. doi: 10.1093/ajcn/53.3.681. PubMed PMID: 2000822.

15. Wolever TM, Brighenti F, Royall D, Jenkins AL, Jenkins DJ. Effect of rectal infusion of short chain fatty acids in human subjects. Am J Gastroenterol. 1989;84(9):1027-33. Epub 1989/09/01. PubMed PMID: 2773895.

16. Chambers ES, Viardot A, Psichas A, Morrison DJ, Murphy KG, ZacVarghese SE, et al. Effects of targeted delivery of propionate to the human colon on appetite regulation, body weight maintenance and adiposity in overweight adults. Gut. 2015;64(11):1744-54. Epub 2014/12/17. doi: 10.1136/gutjnl-2014-307913. PubMed PMID: 25500202; PubMed Central PMCID: PMCPMC4680171.

17. Perry RJ, Peng L, Barry NA, Cline GW, Zhang D, Cardone RL, et al. Acetate mediates a microbiome-brain- $\beta$-cell axis to promote metabolic syndrome. Nature. 2016;534(7606):213-7. doi: 10.1038/nature18309. PubMed PMID: 27279214.

18. Rahat-Rozenbloom S, Fernandes J, Gloor GB, Wolever TM. Evidence for greater production of colonic short-chain fatty acids in overweight than lean humans. International journal of obesity (2005). 2014;38(12):1525-31. Epub 2014/03/20. doi: 10.1038/ijo.2014.46. PubMed PMID: 24642959; PubMed Central PMCID: PMCPMC3970979.

19. Teixeira TF, Grzeskowiak L, Franceschini SC, Bressan J, Ferreira CL, Peluzio MC. Higher level of faecal SCFA in women correlates with metabolic syndrome risk factors. Br J Nutr. 2013;109(5):914-9. Epub 2012/12/04. doi: 10.1017/s0007114512002723. PubMed PMID: 23200109.

20. Turnbaugh PJ, Ley RE, Mahowald MA, Magrini V, Mardis ER, Gordon JI. An obesity-associated gut microbiome with increased capacity for energy harvest. Nature. 2006;444(7122):1027-31. Epub 2006/12/22. doi: 10.1038/nature05414. PubMed PMID: 17183312.

21. Murphy EF, Cotter PD, Healy S, Marques TM, O'Sullivan O, Fouhy $\mathrm{F}$, et al. Composition and energy harvesting capacity of the gut microbiota: relationship to diet, obesity and time in mouse models. Gut. 
2010;59(12):1635-42. Epub 2010/10/12. doi: 10.1136/gut.2010.215665. PubMed PMID: 20926643.

22. McNeil NI, Cummings JH, James WP. Short chain fatty acid absorption by the human large intestine. Gut. 1978;19(9):819-22. PubMed PMID: PMC1412179.

23. Topping DL, Clifton PM. Short-chain fatty acids and human colonic function: roles of resistant starch and nonstarch polysaccharides. Physiological reviews. 2001;81(3):1031-64. Epub 2001/06/28. doi: 10.1152/physrev.2001.81.3.1031. PubMed PMID: 11427691.

24. Ruppin H, Bar-Meir S, Soergel KH, Wood CM, Schmitt MG, Jr. Absorption of short-chain fatty acids by the colon. Gastroenterology. 1980;78(6):1500-7. Epub 1980/06/01. PubMed PMID: 6768637.

25. Rechkemmer G, Rönnau K, Engelhardt WV. Fermentation of polysaccharides and absorption of short chain fatty acids in the mammalian hindgut. Comparative Biochemistry and Physiology Part A: Physiology. 1988;90(4):563-8. doi: 10.1016/0300-9629(88)90668-8.

26. American Diabetes A. Diagnosis and classification of diabetes mellitus. Diabetes care. 2010;33 Suppl 1(Suppl 1):S62-S9. doi: 10.2337/dc10S062. PubMed PMID: 20042775.

27. Canfora EE, van der Beek CM, Hermes GDA, Goossens GH, Jocken JWE, Holst JJ, et al. Supplementation of Diet With Galacto-oligosaccharides Increases Bifidobacteria, but Not Insulin Sensitivity, in Obese Prediabetic Individuals. Gastroenterology. 2017;153(1):87-97.e3. Epub 2017/04/12. doi: 10.1053/j.gastro.2017.03.051. PubMed PMID: 28396144.

28. Reijnders D, Goossens GH, Hermes GD, Neis EP, van der Beek CM, Most J, et al. Effects of Gut Microbiota Manipulation by Antibiotics on Host Metabolism in Obese Humans: A Randomized Double-Blind PlaceboControlled Trial. Cell metabolism. 2016;24(1):63-74. Epub 2016/07/15. doi: 10.1016/j.cmet.2016.06.016. PubMed PMID: 27411009.

29. Müller M, Hermes, G., Canfora, E. E., Holst, J.J., Zoetendal, E. G., Smidt, H., Troost, F., Lenaerts, K., Masclee, A.A.M., Blaak, E. E. The effect of long term Arabinoxylan-oligosaccharide supplementation on gastrointestinal functioning and metabolic parameters: A randomized controlled trial. European Congress on Obesity; 2018; Vienna: Obesity Facts; 2018.

30. Frayn KN. Calculation of substrate oxidation rates in vivo from gaseous exchange. J Appl Physiol Respir Environ Exerc Physiol. 1983;55(2):628-34. Epub 1983/08/01. doi: 10.1152/jappl.1983.55.2.628. PubMed PMID: 6618956.

31. Weir JBdV. New methods for calculating metabolic rate with special reference to protein metabolism. J Physiol. 1949;109(1-2):1-9. PubMed PMID: PMC1392602. 
32. DeFronzo RA, Tobin JD, Andres R. Glucose clamp technique: a method for quantifying insulin secretion and resistance. Am J Physiol. 1979;237(3):E214-23. Epub 1979/09/01. doi: 10.1152/ajpendo.1979.237.3.E214. PubMed PMID: 382871.

33. Garcia-Villalba R, Gimenez-Bastida JA, Garcia-Conesa MT, TomasBarberan FA, Carlos Espin J, Larrosa M. Alternative method for gas chromatography-mass spectrometry analysis of short-chain fatty acids in faecal samples. J Sep Sci. 2012;35(15):1906-13. Epub 2012/08/07. doi: 10.1002/jssc.201101121. PubMed PMID: 22865755.

34. van Eijk HM, Bloemen JG, Dejong $\mathrm{CH}$. Application of liquid chromatography-mass spectrometry to measure short chain fatty acids in blood. Journal of chromatography B, Analytical technologies in the biomedical and life sciences. 2009;877(8-9):719-24. Epub 2009/02/24. doi: 10.1016/j.jchromb.2009.01.039. PubMed PMID: 19230798.

35. Orskov C, Rabenhoj L, Wettergren A, Kofod H, Holst JJ. Tissue and plasma concentrations of amidated and glycine-extended glucagon-like peptide I in humans. Diabetes. 1994;43(4):535-9. Epub 1994/04/01. doi: 10.2337/diab.43.4.535. PubMed PMID: 8138058.

36. Schols AM, Buurman WA, Staal van den Brekel AJ, Dentener MA, Wouters EF. Evidence for a relation between metabolic derangements and increased levels of inflammatory mediators in a subgroup of patients with chronic obstructive pulmonary disease. Thorax. 1996;51(8):819-24. doi: 10.1136/thx.51.8.819.

37. Matthews DR, Hosker JP, Rudenski AS, Naylor BA, Treacher DF, Turner RC. Homeostasis model assessment: insulin resistance and beta-cell function from fasting plasma glucose and insulin concentrations in man. Diabetologia. 1985;28(7):412-9. Epub 1985/07/01. doi: 10.1007/bf00280883. PubMed PMID: 3899825.

38. de la Cuesta-Zuluaga J, Mueller NT, Alvarez-Quintero R, VelasquezMejia EP, Sierra JA, Corrales-Agudelo V, et al. Higher Fecal Short-Chain Fatty Acid Levels Are Associated with Gut Microbiome Dysbiosis, Obesity, Hypertension and Cardiometabolic Disease Risk Factors. Nutrients. 2018;11(1). Epub 2018/12/29. doi: 10.3390/nu11010051. PubMed PMID: 30591685; PubMed Central PMCID: PMCPMC6356834.

39. Vogt JA, Wolever TM. Fecal acetate is inversely related to acetate absorption from the human rectum and distal colon. J Nutr. 2003;133(10):3145-8. Epub 2003/10/02. doi: 10.1093/jn/133.10.3145. PubMed PMID: 14519799.

40. den Besten G, Havinga R, Bleeker A, Rao S, Gerding A, van Eunen $\mathrm{K}$, et al. The short-chain fatty acid uptake fluxes by mice on a guar gum supplemented diet associate with amelioration of major biomarkers of the metabolic syndrome. PLoS One. 2014;9(9):e107392-e. doi: 10.1371/journal.pone.0107392. PubMed PMID: 25203112. 
41. Kaji I, Karaki S, Kuwahara A. Short-chain fatty acid receptor and its contribution to glucagon-like peptide-1 release. Digestion. 2014;89(1):31-6. Epub 2014/01/25. doi: 10.1159/000356211. PubMed PMID: 24458110.

42. Freeland KR, Wilson C, Wolever TM. Adaptation of colonic fermentation and glucagon-like peptide- 1 secretion with increased wheat fibre intake for 1 year in hyperinsulinaemic human subjects. Br J Nutr. 2010;103(1):82-90. Epub 2009/08/12. doi: 10.1017/s0007114509991462. PubMed PMID: 19664300.

43. Christiansen CB, Gabe MBN, Svendsen B, Dragsted LO, Rosenkilde MM, Holst JJ. The impact of short-chain fatty acids on GLP-1 and PYY secretion from the isolated perfused rat colon. American Journal of Physiology-Gastrointestinal and Liver Physiology. 2018;315(1):G53-G65. doi: 10.1152/ajpgi.00346.2017. PubMed PMID: 29494208.

44. Chambers AP, Sorrell JE, Haller A, Roelofs K, Hutch CR, Kim K-S, et al. The Role of Pancreatic Preproglucagon in Glucose Homeostasis in Mice. Cell metabolism. 2017;25(4):927-34.e3. doi: 10.1016/j.cmet.2017.02.008.

45. Gromada J, Chabosseau P, Rutter GA. The $\alpha$-cell in diabetes mellitus. Nature Reviews Endocrinology. 2018;14(12):694-704. doi: 10.1038/s41574018-0097-y.

46. Larraufie P, Martin-Gallausiaux C, Lapaque N, Dore J, Gribble FM, Reimann F, et al. SCFAs strongly stimulate PYY production in human enteroendocrine cells. Scientific Reports. 2018;8(1):74. doi: 10.1038/s41598017-18259-0.

47. Canfora EE, Blaak EE. Acetate: a diet-derived key metabolite in energy metabolism: good or bad in context of obesity and glucose homeostasis? Curr Opin Clin Nutr Metab Care. 2017. Epub 2017/08/11. doi: 10.1097/mco.0000000000000408. PubMed PMID: 28795972.

48. Ge H, Li X, Weiszmann J, Wang P, Baribault H, Chen J-L, et al. Activation of G Protein-Coupled Receptor 43 in Adipocytes Leads to Inhibition of Lipolysis and Suppression of Plasma Free Fatty Acids. Endocrinology. 2008;149(9):4519-26. doi: 10.1210/en.2008-0059.

49. Fernandes J, Vogt J, Wolever TM. Intravenous acetate elicits a greater free fatty acid rebound in normal than hyperinsulinaemic humans. Eur J Clin Nutr. 2012;66(9):1029-34. Epub 2012/07/26. doi: 10.1038/ejcn.2012.98. PubMed PMID: 22828730; PubMed Central PMCID: PMCPMC3937122.

50. Jocken JWE, González Hernández MA, Hoebers NTH, van der Beek CM, Essers YPG, Blaak EE, et al. Short-Chain Fatty Acids Differentially Affect Intracellular Lipolysis in a Human White Adipocyte Model. Front Endocrinol (Lausanne). 2018;8(372). doi: 10.3389/fendo.2017.00372.

51. Girousse A, Tavernier G, Valle C, Moro C, Mejhert N, Dinel A-L, et al. Partial inhibition of adipose tissue lipolysis improves glucose metabolism and insulin sensitivity without alteration of fat mass. PLoS biology. 2013;11(2):e1001485. 
52. Al-Lahham S, Roelofsen H, Rezaee F, Weening D, Hoek A, Vonk R, et al. Propionic acid affects immune status and metabolism in adipose tissue from overweight subjects. Eur J Clin Invest. 2012;42(4):357-64. Epub 2011/09/15. doi: 10.1111/j.1365-2362.2011.02590.x. PubMed PMID: 21913915. 53. Rumberger JM, Arch JRS, Green A. Butyrate and other short-chain fatty acids increase the rate of lipolysis in 3T3-L1 adipocytes. PeerJ. 2014;2:e611-e. doi: 10.7717/peerj.611. PubMed PMID: 25320679.

54. Khan S, Jena G. Sodium butyrate reduces insulin-resistance, fat accumulation and dyslipidemia in type-2 diabetic rat: A comparative study with metformin. Chem Biol Interact. 2016;254:124-34. Epub 2016/06/09. doi: 10.1016/j.cbi.2016.06.007. PubMed PMID: 27270450.

55. Khan S, Jena GB. Protective role of sodium butyrate, a HDAC inhibitor on beta-cell proliferation, function and glucose homeostasis through modulation of p38/ERK MAPK and apoptotic pathways: study in juvenile diabetic rat. Chem Biol Interact. 2014;213:1-12. Epub 2014/02/18. doi: 10.1016/j.cbi.2014.02.001. PubMed PMID: 24530320.

56. Qin J, Li Y, Cai Z, Li S, Zhu J, Zhang F, et al. A metagenome-wide association study of gut microbiota in type 2 diabetes. Nature. 2012;490:55. doi: 10.1038/nature11450.

57. Karlsson FH, Tremaroli V, Nookaew I, Bergström G, Behre CJ, Fagerberg B, et al. Gut metagenome in European women with normal, impaired and diabetic glucose control. Nature. 2013;498:99. doi: 10.1038/nature12198.

58. Brahe LK, Astrup A, Larsen LH. Is butyrate the link between diet, intestinal microbiota and obesity-related metabolic diseases? Obesity Reviews. 2013;14(12):950-9. doi: 10.1111/obr.12068.

59. Layden BT, Yalamanchi SK, Wolever TM, Dunaif A, Lowe WL, Jr. Negative association of acetate with visceral adipose tissue and insulin levels. Diabetes, metabolic syndrome and obesity : targets and therapy. 2012;5:49-55. Epub 2012/03/16. doi: 10.2147/dmso.S29244. PubMed PMID: 22419881 ; PubMed Central PMCID: PMCPMC3299553.

60. Moreno-Navarrete JM, Serino M, Blasco-Baque V, Azalbert V, Barton RH, Cardellini M, et al. Gut Microbiota Interacts with Markers of Adipose Tissue Browning, Insulin Action and Plasma Acetate in Morbid Obesity. Mol Nutr Food Res. 2018;62(3). Epub 2017/11/07. doi: 10.1002/mnfr.201700721. PubMed PMID: 29105287.

61. Fernandes J, Vogt J, Wolever TMS. Inulin increases short-term markers for colonic fermentation similarly in healthy and hyperinsulinaemic humans. European Journal Of Clinical Nutrition. 2011;65:1279. doi: 10.1038/ejcn.2011.116.

62. Fernandes J, Vogt J, Wolever TMS. Kinetic model of acetate metabolism in healthy and hyperinsulinaemic humans. European Journal of Clinical Nutrition. 2014;68(9):1067-71. doi: 10.1038/ejcn.2014.136. 
63. Han J-H, Kim I-S, Jung S-H, Lee S-G, Son H-Y, Myung C-S. The effects of propionate and valerate on insulin responsiveness for glucose uptake in 3T3-L1 adipocytes and C2C12 myotubes via G protein-coupled receptor 41. PLoS One. 2014;9(4):e95268-e. doi: 10.1371/journal.pone.0095268. PubMed PMID: 24748202.

64. Weitkunat K, Schumann S, Nickel D, Kappo KA, Petzke KJ, Kipp AP, et al. Importance of propionate for the repression of hepatic lipogenesis and improvement of insulin sensitivity in high-fat diet-induced obesity. Mol Nutr Food Res. 2016;60(12):2611-21. Epub 2016/07/29. doi: 10.1002/mnfr.201600305. PubMed PMID: 27467905; PubMed Central PMCID: PMCPMC5215627.

65. Weitkunat K, Schumann S, Nickel D, Hornemann S, Petzke KJ, Schulze $\mathrm{MB}$, et al. Odd-chain fatty acids as a biomarker for dietary fiber intake: a novel pathway for endogenous production from propionate. The American Journal of Clinical Nutrition. 2017;105(6):1544-51. doi: 10.3945/ajen.117.152702.

66. Knowles SE, Jarrett IG, Filsell OH, Ballard FJ. Production and utilization of acetate in mammals. Biochem J. 1974;142(2):401-11. Epub 1974/08/01. doi: 10.1042/bj1420401. PubMed PMID: 4441381; PubMed Central PMCID: PMCPMC1168292.

67. Sun EW, de Fontgalland D, Rabbitt P, Hollington P, Sposato L, Due SL, et al. Mechanisms Controlling Glucose-Induced GLP-1 Secretion in Human Small Intestine. Diabetes. 2017;66(8):2144-9. doi: 10.2337/db17-0058. 


\section{Supplementary Material}

A

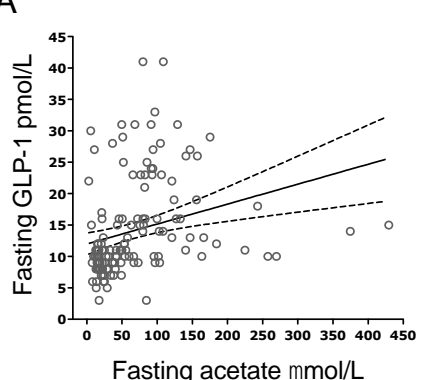

D

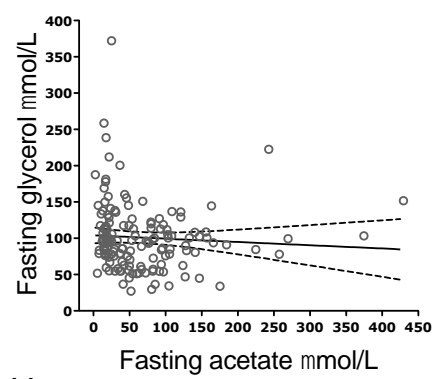

$\mathrm{H}$

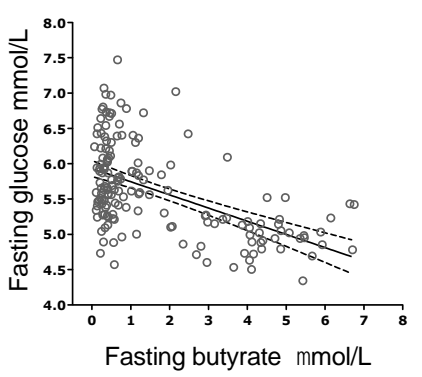

K

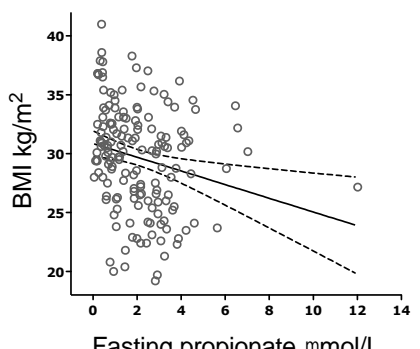

B

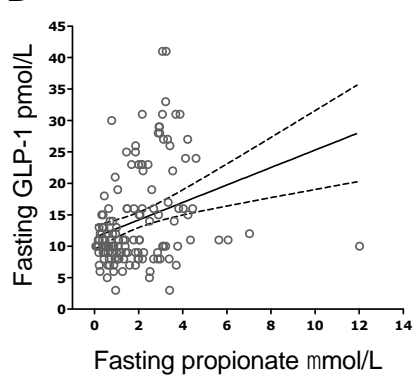

$\mathrm{F}$

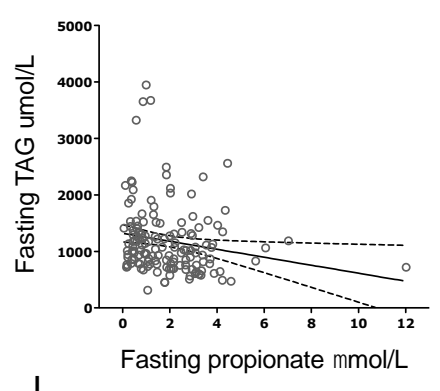

I

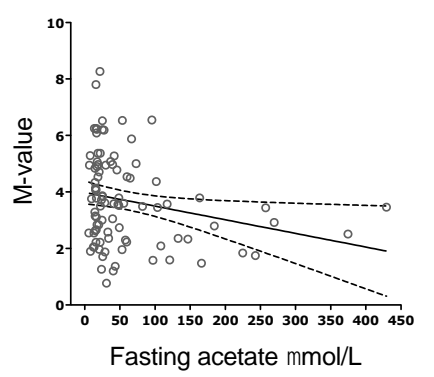

$\mathrm{L}$

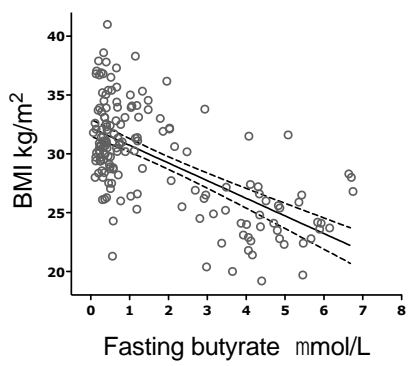

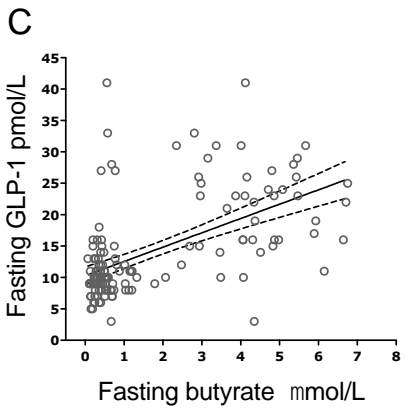

G
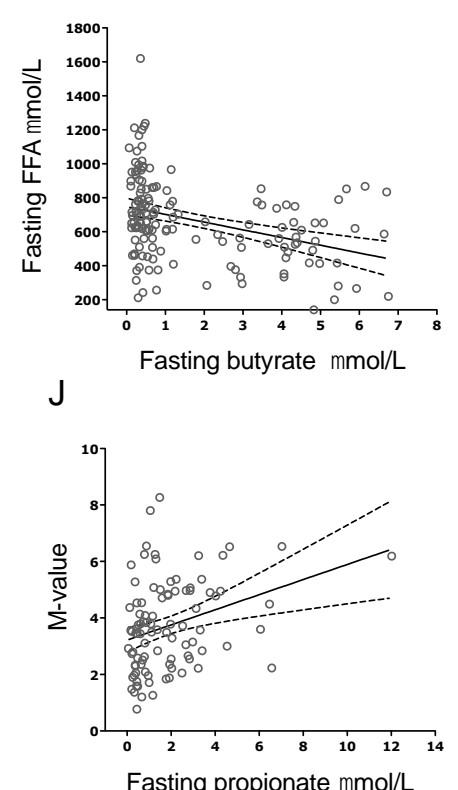

Supplementary Figure 1 Associations between metabolic parameters, BMI and insulin sensitivity and fasting circulating SCFA. Linear regression scatter plots of fasting circulating SCFA with fasting GLP-1 (A-C), fasting acetate with fasting glycerol (D), fasting propionate with fasting TAG (F), fasting butyrate with FFA (G) and fasting glucose $(\mathbf{H})$, fasting acetate with $\mathrm{M}$-value (I), fasting propionate with M-value (J) and BMI (K) and fasting butyrate with BMI (L). GLP-1 glucagon like peptide 1, TAG triacylglycerol, FFA free fatty acids, BMI body mass index 




\section{CHAPTER 5}

Fecal microbial metabolites of proteolytic and saccharolytic fermentation in relation to degree of insulin resistance in overweight and obese individuals

Running title: Microbial metabolites, obesity and insulin resistance

Manuel A. González Hernández, Emanuel E. Canfora and Ellen E. Blaak.

To be submitted 


\begin{abstract}
The gut microbiota may affect host metabolic health through microbial metabolites. The balance between the production of microbial metabolites by saccharolytic and proteolytic fermentation may be an important determinant of metabolic health. Amongst the best-studied saccharolytic microbial metabolites are the short-chain fatty acids acetate, propionate and butyrate. However, human data on the role of other microbial fermentation byproducts in metabolic health are greatly lacking. Therefore, we compared in a cross-sectional study the fecal microbial metabolites (caproate, lactate, valerate, succinate, and the branchedchain fatty acids (BCFA) (isobutyrate, isovalerate)) between insulin sensitive (HOMA-IR $<1.85$, IS) and insulin resistant (HOMA-IR $>1.85$, IR) individuals. Additionally, we assessed the relationships between fecal metabolites and markers of metabolic health including fasting glucose, insulin, free fatty acids, insulin resistance ((homeostatic model assessment of insulin resistance (HOMAIR)) and fasting substrate oxidation in 86 individuals with a wide range of body mass (BMI). Fecal metabolite concentrations did not significantly differ between IS and IR. Furthermore, there were no associations between microbial metabolites and metabolic health markers, except for a slight positive association of isovalerate with carbohydrate oxidation ( $\mathrm{E} \%$, std $\beta 0.194, \mathrm{P}=0.011)$ and fat oxidation $(\mathrm{E} \%$, std $\beta$ $-0.075, \mathrm{P}=0.047)$, also after adjustment for age, sex and BMI. In summary, fecal caproate, lactate, valerate, succinate, and BCFA (isobutyrate, isovalerate) were not different between IR and IS individuals, nor was there any association between these fecal metabolites and parameters of metabolic health. Further human intervention studies are warranted to investigate the role of these microbiallyderived fermentation products and their kinetics in metabolic health and insulin sensitivity.
\end{abstract}




\section{Introduction}

Metabolic health is impacted by gut health maintenance which is intricately modulated through various mechanisms, including the preservation of a balanced microbial composition and functionality ${ }^{1,2}$. In addition, a high microbial diversity has been linked to gut health as well as to an improved insulin sensitivity and metabolic health in various animal and human studies ${ }^{3-8}$. In contrast, the presence of gut dysbiosis has been linked to the development of obesity and its complications ${ }^{9}$.

Metabolites derived from the microbial fermentation of indigestible foods play an important role in the microbes-host metabolic crosstalk. In the proximal colon, microbial fermentation of mainly indigestible carbohydrates (saccharolytic fermentation) produces short chain fatty acids (SCFA, acetate, propionate and butyrate) as well as other metabolites (i.e. lactate, succinate, valerate, caproate) and gases (i.e. methane, carbon dioxide and hydrogen) ${ }^{2}{ }^{10}$. The distal portion of the colon specializes more in proteolytic fermentation (residual peptides and proteins) and produces a wide variety of metabolites including phenolic compounds, indoles, hydrogen sulfide as well as branched chain fatty acids (BCFA) such as isovalerate and isobutyrate ${ }^{1,11,12}$. In general, the majority of microbial metabolites derived from proteolytic fermentation are associated with adverse gut and metabolic health effects. An increased production of proteolytic fermentation byproducts elicited by high protein diets (HPD) or low-fiber diets may lead to altered production of microbial derived metabolites (i.e. increase fecal BCFA and decrease fecal SCFA), alterations in gut microbiota, colonic epithelium as well as impaired detoxification capacity of gut mucosa and increased inflammation ${ }^{13}$.

Furthermore, in human small-scale observational studies, fecal BCFA concentrations have been reported to be higher in individuals with obesity ${ }^{14}$ and hypercholesterolemia ${ }^{15}$ as well as associated to NAFLD progression ${ }^{16}$. Interestingly, the gut microbiome from obese individuals has shown a higher biosynthetic capacity for BCFA precursors, branched chain amino acids ${ }^{17}$, which circulating levels have been related to tissue specific insulin resistance in obese individuals ${ }^{18}$ as well as with T2DM ${ }^{19,20}$. Currently, there is a lack of studies that investigate the role of microbial fermentation byproducts besides the major SCFA acetate, propionate and butyrate. In the present cross-sectional study, we investigated the comparisons of fecal microbial metabolites (caproate, lactate, valerate, succinate, and the BCFA (isobutyrate, isovalerate)) between insulin sensitive (HOMA-IR<1.85, IS) and insulin resistant (HOMA-IR>1.85, IR) individuals. Secondly, associations of these fecal metabolites with fasting glucose, insulin, free fatty acids (FFA), insulin resistance ((homeostatic model assessment of insulin resistance (HOMA-IR)) and fasting substrate oxidation were assessed in the whole cohort. 


\section{Methods}

\section{Study participants}

A cohort of 86 Caucasian men and women aged 20-69 years with a BMI between 19.2 and $35.2 \mathrm{~kg} / \mathrm{m}^{2}$ from the vicinity of Maastricht was divided based on the HOMA-IR median (1.85) in insulin sensitive (IS, HOMA-IR<1.85) and insulin resistant individuals (IR, HOMA-IR $>1.85$ ). Additionally, in the total cohort, associations between fecal metabolites and parameters of metabolic health were assessed. Eligibility of the participants was assessed via a general health questionnaire, medical history and anthropometry during an initial screening visit. Exclusion criteria were as follows: use of antibiotics, prebiotics, or probiotics 3 months before or during the study, diagnosis of T2DM, gastrointestinal or cardiovascular diseases, abdominal surgery, participants with life expectancy shorter than 5 years, alcohol or drug abuse and participants following a hypocaloric diet. Participants did not use $\beta$-blockers, lipid- or glucose-lowering drugs, antioxidants, or chronic corticosteroids. All protocols were reviewed and approved by the local Medical Ethical Committee (MUMC+) and conducted in accordance with the Declaration of Helsinki (revised version, October 2008, Seoul, South Korea). Written informed consent was obtained from all participants.

\section{Study Design}

This cross-sectional analysis included metabolic parameters as well as fecal metabolites (caproate, lactate, valerate, succinate, BCFA) of previously performed interventions ${ }^{21-23}$. In the present study, we collated and analyzed study data at baseline and thus prior to the respective interventions. In all studies, sample collection and measurements were conducted according to the same standard operating procedures. Two days prior to the baseline investigation day, participants were asked to refrain from intense physical activity and alcohol consumption. On the evening before the investigation day, subjects were provided with a standardized meal composed of $65 \mathrm{E} \%$ carbohydrates, $20 \mathrm{E} \%$ fat and $15 \mathrm{E} \%$ protein.

\section{Used data sets}

For this analysis we included baseline data from the following intervention human in vivo studies. The data set includes an intervention study with arabinoxylan-oligosacharide (AXOS) in healthy participants with slow gastrointestinal transit (Clinical trial No. NCT02491125) ${ }^{22}$, an intervention study with prebiotic inulin in overweight to obese individuals on substrate metabolism (Clinical trial No. NCT03711383) ${ }^{21}$ and an intervention study with beta glucan in lean-overweight individuals (Clinical trial No. NCT03714646) ${ }^{24}$. 


\section{Baseline investigation day}

After an overnight fast ( $>10 \mathrm{~h})$, participants came to the laboratory by car or public transport. Anthropometry was measured including height, weight and waist to hip ratio. After inserting a cannula into the antecubital vein, blood samples were taken to measure plasma metabolites in the fasted state. After the blood sampling, participants were in a resting, half-supine position and fasting substrate oxidation was measured for 30 min using an open circuit ventilated hood system (Omnical, MUMC+, Maastricht, the Netherlands).

Fat and carbohydrate oxidation were calculated according to the equations of Weir and Frayn ${ }^{25,26}$, assuming that protein oxidation accounted for $15 \%$ of total energy expenditure. Fat and carbohydrate oxidation are expressed as percentage of energy expenditure.

\section{Analysis of fecal microbial metabolites}

Fecal samples were collected at home and stored in the subjects' freezer at $-20{ }^{\circ} \mathrm{C}$ maximum of two days before the baseline investigation day, transported on dry ice, and stored on arrival at the university at $-80^{\circ} \mathrm{C}$. BCFA, lactate, succinate, caproate and valerate were measured using gas chromatography-mass spectrometry as described previously ${ }^{27}$.

\section{Blood collection and biochemical analysis}

Blood was collected in pre-chilled EDTA tubes (0.2 mol/L EDTA; Sigma, Dorset, UK) for insulin, glucose, FFA, analyses during fasting conditions. Plasma glucose and FFA were determined using commercially available reagent kits for glucose (Glucose HK CP, Horiba ABX Pentra, Montpellier, France) and FFA (NEFA-HR (2) assay, Wako, Sopachem BV, Ochten, the Netherlands). Plasma insulin was determined with a commercially available radioimmunoassay (RIA) kit (HI-14K Human Insulin specific RIA HI-14K, Millipore Merck) according to the manufacture's protocol. HOMA-IR was calculated as the product of (glucose $(\mathrm{mmol} / \mathrm{L}){ }^{*}$ insulin $\left.(\mu \mathrm{U} / \mathrm{mL}) / 22.5\right)$, using fasting values ${ }^{28}$.

\section{Statistical analysis}

First, we divided the total cohort into two groups based on the HOMA-IR median (1.85), insulin sensitive (IS, HOMA-IR<1.85) and insulin resistant individuals (IR, HOMA-IR>1.85) to compare the microbial metabolites on group level. The normality of data was assessed with the Gaussian distribution and Kolmogorov-Smirnov procedure. Variables that were not normally distributed were $\ln$ - transformed. In case of missing data, the participant was excluded from the analysis. Differences between IS and IR characteristics were analyzed using a Student's independent samples t-test. Next, we used simple/multiple linear regression to investigate the associations between fecal metabolites (as independent variables) and metabolic parameters (as dependent variables), 
insulin resistance (HOMA-IR), circulating glucose, insulin, circulating FFA and fasting substrate oxidation in the whole cohort. Multiple linear regression models were adjusted for the covariates age, sex and BMI. All data were analysed using SPSS 22.0 (IBM, Armok, U.S.) with significance set at $\mathrm{P}<0.05$.

\section{Results}

Table 1. Characteristics of Insulin sensitive (HOMA-IR<1.85) and Insulin resistant (HOMAIR>1.85) individuals

\begin{tabular}{|c|c|c|c|c|c|}
\hline \multirow[b]{2}{*}{ Parameter } & \multicolumn{2}{|c|}{ IS $(\mathrm{N}=43)$} & \multicolumn{2}{|c|}{ IR $(\mathrm{N}=43)$} & \multirow{2}{*}{$\begin{array}{l}\mathbf{P} \\
\text { value }\end{array}$} \\
\hline & $\begin{array}{l}\text { Mean } \\
\pm \text { SD }\end{array}$ & Range & $\begin{array}{l}\text { Mean } \\
\pm \text { SD }\end{array}$ & Range & \\
\hline $\operatorname{Sex}(F / M)$ & \multicolumn{2}{|l|}{$18 / 25$} & \multicolumn{2}{|l|}{$16 / 27$} & \\
\hline Age (y) & $\begin{array}{l}46.26 \\
\pm 15\end{array}$ & $20-66$ & $\begin{array}{l}45.6 \pm \\
16\end{array}$ & $20-69$ & 0.850 \\
\hline Body weight (kg) & $\begin{array}{l}73.7 \pm \\
11.3\end{array}$ & $52-103$ & $\begin{array}{l}83.8 \pm \\
15.2\end{array}$ & $57-144$ & 0.000 \\
\hline BMI $\left(\mathrm{kg} / \mathrm{m}^{2}\right)$ & $\begin{array}{l}24 \pm \\
2.18\end{array}$ & $19-28$ & $\begin{array}{l}27.1 \pm \\
3.6\end{array}$ & $19-35$ & 0.000 \\
\hline Waist: Hip ratio & $\begin{array}{l}0.85 \pm \\
0.11\end{array}$ & $\begin{array}{l}0.64- \\
1.07\end{array}$ & $\begin{array}{l}0.89 \pm \\
0.12\end{array}$ & $\begin{array}{l}0.70- \\
1.07\end{array}$ & 0.123 \\
\hline HOMA-IR & $\begin{array}{l}1.28 \pm \\
0.33\end{array}$ & $\begin{array}{l}0.56- \\
1.85\end{array}$ & $\begin{array}{l}3.01 \pm \\
1.41\end{array}$ & $\begin{array}{l}1.85- \\
8.51\end{array}$ & 0.000 \\
\hline $\begin{array}{l}\text { Fasting plasma insulin } \\
(\mathrm{mU} / \mathrm{L})\end{array}$ & $\begin{array}{l}5.8 \pm \\
1.54\end{array}$ & $2-9$ & $\begin{array}{l}12.33 \\
\pm 5.8\end{array}$ & $7-39$ & 0.000 \\
\hline $\begin{array}{l}\text { Fasting plasma glucose } \\
(\mathrm{mmol} / \mathrm{L})\end{array}$ & $\begin{array}{l}4.9 \pm \\
0.42\end{array}$ & $4-6.3$ & $\begin{array}{l}5.51 \pm \\
0.76\end{array}$ & $4-7.4$ & 0.000 \\
\hline 2h glucose $(\mathrm{mmol} / \mathrm{L})$ & $\begin{array}{l}4.75 \\
\pm 1.76\end{array}$ & $2.1-11$ & $\begin{array}{l}6.16 \pm \\
2.26\end{array}$ & $2-10.8$ & 0.002 \\
\hline $\begin{array}{l}\text { Fasting plasma free fatty } \\
\text { acids }(\mu \mathrm{mol} / \mathrm{L})\end{array}$ & $\begin{array}{l}517 \pm \\
214\end{array}$ & $\begin{array}{l}117- \\
949\end{array}$ & $\begin{array}{l}468 \pm \\
146\end{array}$ & $96-759$ & 0.222 \\
\hline Fecal isobutyrate $(\mu \mathrm{mol} / \mathrm{g})$ & $\begin{array}{l}1.81 \pm \\
0.94\end{array}$ & $0.6-4.3$ & $\begin{array}{l}1.95 \pm \\
1.30\end{array}$ & $0.1-5.4$ & 0.586 \\
\hline Fecal valerate $(\mu \mathrm{mol} / \mathrm{g})$ & $\begin{array}{l}1.73 \pm \\
1.46\end{array}$ & $\begin{array}{l}0.14- \\
8.43\end{array}$ & $\begin{array}{l}1.79 \pm \\
1.47\end{array}$ & $\begin{array}{l}0.1- \\
5.28\end{array}$ & 0.857 \\
\hline Fecal isovalerate $(\mu \mathrm{mol} / \mathrm{g})$ & $\begin{array}{l}2.68 \pm \\
1.56\end{array}$ & $\begin{array}{l}0.1- \\
6.26\end{array}$ & $\begin{array}{l}2.67 \pm \\
2.55\end{array}$ & $\begin{array}{l}0.1- \\
12.76\end{array}$ & 0.988 \\
\hline Fecal caproate $(\mu \mathrm{mol} / \mathrm{g})$ & $\begin{array}{l}0.97 \pm \\
0.84\end{array}$ & $\begin{array}{l}0.1- \\
3.10\end{array}$ & $\begin{array}{l}1.29 \pm \\
1.49\end{array}$ & $\begin{array}{l}0.1- \\
7.11\end{array}$ & 0.314 \\
\hline Fecal succinate $(\mu \mathrm{mol} / \mathrm{g})$ & $\begin{array}{l}1.27 \pm \\
0.97\end{array}$ & $0-3.55$ & $\begin{array}{l}1.79 \pm \\
2.62\end{array}$ & $\begin{array}{l}0.1- \\
15.3\end{array}$ & 0.289 \\
\hline Fecal lactate $(\mu \mathrm{mol} / \mathrm{g})$ & $\begin{array}{l}0.99 \pm \\
1.1\end{array}$ & $\begin{array}{l}0.1- \\
4.69\end{array}$ & $\begin{array}{l}0.89 \pm \\
0.94\end{array}$ & $\begin{array}{l}0.1- \\
3.32\end{array}$ & 0.706 \\
\hline Energy intake (kcal/d) & $\begin{array}{l}1924 \\
\pm 503\end{array}$ & $\begin{array}{l}1065- \\
3600\end{array}$ & $\begin{array}{l}1957 \pm \\
471\end{array}$ & $\begin{array}{l}1265- \\
3165\end{array}$ & 0.786 \\
\hline Carbohydrate intake, E\% & $46 \pm 7$ & $32-63$ & $46 \pm 6$ & $34-60$ & 0.905 \\
\hline Fat intake, E\% & $34 \pm 7$ & $16-50$ & $35 \pm 6$ & $23-49$ & 0.455 \\
\hline Protein intake, E\% & $17 \pm 4$ & $10-27$ & $17 \pm 4$ & $9-25$ & 0.610 \\
\hline Dietary fiber (g/MJ) & $\begin{array}{l}2.38 \pm \\
0.81\end{array}$ & $\begin{array}{l}1.08- \\
4.06\end{array}$ & $\begin{array}{l}2.39 \pm \\
0.57\end{array}$ & $\begin{array}{l}1.38- \\
4.33\end{array}$ & 0.948 \\
\hline
\end{tabular}


The mean age of the participants in our cohort was $45.8 \pm 15$ years and $60.5 \%$ of participants were male. To study differences between the aforementioned fecal metabolites including proteolytic fermentation byproducts (BCFA) in relation to insulin sensitivity on group level, we divided the total cohort into two groups based on the HOMA-IR median (1.85), insulin sensitive (IS, HOMA-IR<1.85) and insulin resistant individuals (IR, HOMA-IR>1.85). While there were differences between groups in body weight, BMI, fasting insulin, fasting glucose, 2-h glucose and HOMA-IR, there were no differences in fecal metabolites between IS and IR groups (Table 1).

We found a weak association of fecal isovalerate concentrations with carbohydrate oxidation $\mathrm{E} \%$ (std $\beta 0.194 \mathrm{P}$-value $=0.011)$ and fat oxidation $\mathrm{E} \%$ (std $\beta$ $-0.075 \mathrm{P}$-value $=0.047$ ) in the whole cohort (Table 2), which was maintained after adjustment for age, sex and BMI. However, we did not find further associations between the fecal metabolites valerate, caproate, succinate, lactate and BCFA concentrations with fasting metabolites (glucose, insulin, FFA), HOMA-IR and fasting substrate oxidation in the whole cohort.

\begin{tabular}{|c|c|c|c|c|c|c|c|c|c|c|c|c|}
\hline \multirow[b]{2}{*}{ Metabolite } & \multicolumn{4}{|c|}{ Fecal isobutyrate } & \multicolumn{4}{|c|}{ Fecal valerate } & \multicolumn{4}{|c|}{ Fecal isovalerate } \\
\hline & $\operatorname{std} \beta$ & $P$ & $\begin{array}{ll}\text { std } & \beta \\
\text { adj } & \end{array}$ & $P$ & $\operatorname{std} \beta$ & $P$ & $\begin{array}{ll}\text { std } & \beta \\
\text { adj } & \end{array}$ & $P$ & $\operatorname{std} \beta$ & $P$ & $\begin{array}{ll}\text { std } & \beta \\
\text { adj } & \end{array}$ & $P$ \\
\hline $\begin{array}{l}\text { FFA, } \\
\mu \mathrm{mol} / \mathrm{L}\end{array}$ & $\begin{array}{l}-0.079 \pm \\
0.061\end{array}$ & 0.196 & $\begin{array}{l}-0.070 \\
\pm 0.056\end{array}$ & 0.211 & $\begin{array}{l}-.008 \pm \\
0.045\end{array}$ & 0.858 & $\begin{array}{l}0.015 \pm \\
0.042\end{array}$ & 0.713 & $\begin{array}{l}-.018 \pm \\
0.053\end{array}$ & 0.742 & $\begin{array}{l}-0.029 \\
\pm 0.049\end{array}$ & 0.556 \\
\hline $\begin{array}{l}\text { Insulin, } \\
\text { mU/L }\end{array}$ & $\begin{array}{l}-0.007 \\
\pm 0.057\end{array}$ & 0.917 & $\begin{array}{l}0.023 \pm \\
0.056\end{array}$ & 0.686 & $\begin{array}{l}-0.003 \pm \\
0.051\end{array}$ & 0.950 & $\begin{array}{l}-0.005 \\
\pm 0.044\end{array}$ & 0.911 & $\begin{array}{l}-0.030 \\
\pm 0.061\end{array}$ & 0.625 & $\begin{array}{l}-0.014 \\
\pm 0.052\end{array}$ & 0.782 \\
\hline $\begin{array}{l}\text { HOMA- } \\
\text { IR }\end{array}$ & $\begin{array}{l}-0.013 \pm \\
0.071\end{array}$ & 0.855 & $\begin{array}{l}0.026 \pm \\
0.057\end{array}$ & 0.646 & $\begin{array}{l}0.004 \pm \\
0.055\end{array}$ & 0.939 & $\begin{array}{l}-0.004 \\
\pm 0.046\end{array}$ & 0.931 & $\begin{array}{l}-0.028 \\
\pm 0.066\end{array}$ & 0.672 & $\begin{array}{l}0.001 \pm \\
0.054\end{array}$ & 0.979 \\
\hline $\begin{array}{l}\text { Glucose, } \\
\mathrm{mmol} / \mathrm{L}\end{array}$ & $\begin{array}{l}-0.008 \pm \\
0.015\end{array}$ & 0.596 & $\begin{array}{l}0.002 \pm \\
0.013\end{array}$ & 0.865 & $\begin{array}{l}0.006 \pm \\
0.011\end{array}$ & 0.611 & $\begin{array}{l}0.000 \pm \\
0.009\end{array}$ & 0.976 & $\begin{array}{l}0.000 \pm \\
0.014\end{array}$ & 0.982 & $\begin{array}{l}0.015 \pm \\
0.012\end{array}$ & 0.216 \\
\hline Fat, E\% & $\begin{array}{l}-0.078 \pm \\
0.042\end{array}$ & 0.068 & $\begin{array}{l}-0.073 \\
\pm 0.042\end{array}$ & 0.086 & $\begin{array}{l}-0.008 \pm \\
0.032\end{array}$ & 0.813 & $\begin{array}{l}-0.002 \\
\pm 0.032\end{array}$ & 0.957 & $\begin{array}{l}-0.073 \\
\pm 0.037\end{array}$ & 0.047 & $\begin{array}{l}-0.075 \\
\pm 0.037\end{array}$ & 0.047 \\
\hline \multirow[t]{3}{*}{$\begin{array}{l}\text { CHO, } \\
\text { E \% }\end{array}$} & $\begin{array}{l}0.123 \pm \\
0.082\end{array}$ & 0.140 & $\begin{array}{l}0.117 \pm \\
0.083\end{array}$ & 0.167 & $\begin{array}{l}0.045 \pm \\
0.062\end{array}$ & 0.468 & $\begin{array}{l}0.039 \pm \\
0.063\end{array}$ & 0.539 & $\begin{array}{l}0.187 \pm \\
0.074\end{array}$ & 0.012 & $\begin{array}{l}0.194 \pm \\
0.075\end{array}$ & 0.011 \\
\hline & \multicolumn{4}{|c|}{ Fecal caproate } & \multicolumn{4}{|c|}{ Fecal succinate } & \multicolumn{4}{|c|}{ Fecal lactate } \\
\hline & $\operatorname{std} \beta$ & $P$ & $\begin{array}{ll}\text { std } & \beta \\
\text { adj } & \end{array}$ & $P$ & std $\beta$ & $P$ & $\begin{array}{ll}\text { std } & \beta \\
\text { adj } & \end{array}$ & $P$ & $\operatorname{std} \beta$ & $P$ & $\begin{array}{ll}\text { std } & \beta \\
\text { adj } & \end{array}$ & $P$ \\
\hline $\begin{array}{l}\text { FFA, } \\
\mu \mathrm{mol} / \mathrm{L}\end{array}$ & $\begin{array}{l}-0.016 \pm \\
0.046\end{array}$ & 0.731 & $\begin{array}{l}-0.007 \\
\pm 0.043\end{array}$ & 0.866 & $\begin{array}{l}0.003 \pm \\
0.049\end{array}$ & 0.951 & $\begin{array}{l}-0.052 \\
\pm 0.045\end{array}$ & 0.257 & $\begin{array}{l}0.040 \pm \\
0.050\end{array}$ & 0.419 & $\begin{array}{l}-0.032 \\
\pm 0.048\end{array}$ & 0.507 \\
\hline $\begin{array}{l}\text { Insulin, } \\
\text { mU/L }\end{array}$ & $\begin{array}{l}0.092 \pm \\
0.046\end{array}$ & 0.051 & $\begin{array}{l}0.073 \pm \\
0.040\end{array}$ & 0.075 & $\begin{array}{l}0.009 \pm \\
0.052\end{array}$ & 0.867 & $\begin{array}{l}0.001 \pm \\
0.045\end{array}$ & 0.981 & $\begin{array}{l}0.017 \\
\pm 0.052\end{array}$ & 0.745 & $\begin{array}{l}-0.015 \\
\pm 0.048\end{array}$ & 0.747 \\
\hline $\begin{array}{l}\text { HOMA- } \\
\text { IR }\end{array}$ & $\begin{array}{l}0.094 \pm \\
0.048\end{array}$ & 0.057 & $\begin{array}{l}0.070 \pm \\
0.040\end{array}$ & 0.084 & $\begin{array}{l}0.001 \pm \\
0.055\end{array}$ & 0.979 & $\begin{array}{l}0.004 \pm \\
0.045\end{array}$ & 0.929 & $\begin{array}{l}-0.005 \\
\pm .055\end{array}$ & 0.925 & $\begin{array}{l}-0.030 \\
\pm 0.541\end{array}$ & 0.541 \\
\hline $\begin{array}{l}\text { Glucose, } \\
\mathrm{mmol} / \mathrm{L}\end{array}$ & $\begin{array}{l}0.000 \pm \\
0.010\end{array}$ & 0.981 & $\begin{array}{l}-0.003 \\
\pm 0.008\end{array}$ & 0.691 & $\begin{array}{l}-0.008 \pm \\
0.012\end{array}$ & 0.508 & $\begin{array}{l}0.004 \pm \\
0.010\end{array}$ & 0.719 & $\begin{array}{l}-0.023 \\
\pm 0.011\end{array}$ & 0.031 & $\begin{array}{l}-0.015 \\
\pm 0.010\end{array}$ & 0.133 \\
\hline Fat, E\% & $\begin{array}{l}-0.011 \pm \\
0.032\end{array}$ & 0.738 & $\begin{array}{l}-0.011 \\
\pm 0.031\end{array}$ & 0.725 & $\begin{array}{l}-0.015 \pm \\
0.035\end{array}$ & 0.675 & $\begin{array}{l}-0.031 \\
\pm 0.036\end{array}$ & 0.390 & $\begin{array}{l}0.019 \pm \\
0.035\end{array}$ & 0.584 & $\begin{array}{l}-0.006 \\
\pm 0.037\end{array}$ & 0.872 \\
\hline $\begin{array}{l}\text { CHO, } \\
\text { E \% }\end{array}$ & $\begin{array}{l}0.003 \pm \\
0.067\end{array}$ & 0.960 & $\begin{array}{l}0.009 \pm \\
0.057\end{array}$ & 0.892 & $\begin{array}{l}-0.044 \pm \\
0.070\end{array}$ & 0.535 & $\begin{array}{l}-0.033 \\
\pm 0.073\end{array}$ & 0.652 & $\begin{array}{l}-0.032 \\
\pm 0.072\end{array}$ & 0.658 & $\begin{array}{l}0.002 \pm \\
0.078\end{array}$ & 0.976 \\
\hline \multicolumn{13}{|c|}{$\begin{array}{l}\beta \text {, standardized } \beta \text { coefficient }+ \text { standard error of coefficient of fecal branched chain fatty acids (isobutyrate, isovalerate), } \\
\text { valerate, caproate, succinate and lactate as dependent variable in a simple regression and multiple regression analysis } \\
\text { adjusted for age, sex and BMI. Adj, adjusted, std standardized, E\%, percentage of energy expenditure, FFA, free fatty acids, } \\
\text { HOMA-IR, homeostatic model assessment of insulin resistance }\end{array}$} \\
\hline
\end{tabular}




\section{Discussion}

In this cross-sectional analysis, we showed that the fecal levels of BCFA and other fecal metabolites (lactate, succinate, caproate and valerate) were not different between IS and IR individuals. In addition, we could not find a relationship between these microbiota-derived metabolites and insulin resistance and metabolic health parameters in a continuous analysis in the total group.

Our findings are in line with a study that did not find differences in fecal BCFA levels between overweight/obese and healthy individuals ${ }^{29}$. In contrast, another cross-sectional study showed increased total fecal BCFA (isovalerate, isobutyrate and 2 methylbutyrate) and valerate concentrations in obese (30-37 BMI kg/m²) hyperinsulinaemic individuals as compared to non-obese normo-insulinemic individuals ${ }^{14}$. Furthermore, increased fecal isobutyrate concentrations have been previously related to NAFLD progression in both rodents ${ }^{30}$ and humans ${ }^{16}$ and higher fecal BCFA have been associated with hypercholesterolemia ${ }^{15}$. In view of the above inconsistent data, it is important to consider that fecal levels depend on various factors including microbial community ${ }^{31}$, intestinal transit time ${ }^{32}$, diet ${ }^{33}$, 34, age ${ }^{35}$ and lifestyle ${ }^{36}$. Additionally, gut-derived circulating metabolite concentrations may relate more strongly with changes in whole body metabolic markers than fecal concentrations as we have previously suggested for SCFA ${ }^{37}$. Of note, obese individuals have been reported to have lower circulating isobutyrate and higher isovalerate concentrations as compared to healthy individuals ${ }^{38}$. In addition, an in vitro study using rat and human adipocytes showed that both BCFA (isobutyrate and isovalerate) inhibited cytosolic (intracellular) lipolysis and lipogenesis using supraphysiological concentrations $(10 \mathrm{mmol} / \mathrm{L})^{39}$.

Thus, although our study did not show differences in fecal BCFA between metabolic phenotypes, other studies $(38,39)$ may suggest alteration in circulating BCFA in metabolically compromised individuals and possible tissue specific metabolic effects. More research investigating BCFA dynamics may shed light on whether BCFA handling is disturbed in different metabolic phenotypes and/or whether BCFA need to enter the circulation to exert metabolic effects.

Of note, various human studies reported that a HPD could increase fecal BCFA/SCFA ratio through a higher proteolytic fermentation, possibly inducing adverse health consequences in the long-term ${ }^{13}, 40$. Additionally, a human study reported that a high fiber diet (resistant starch type 2 and arabinoxylan fibers) increased saccharolytic/proteolytic fermentation ratio while a Western diet did not 41. Collectively, current literature shows that the capacity of dietary interventions to modulate saccharolytic/proteolytic fermentation may have an impact on gut and metabolic health.

A further interesting aspect to consider in future research is the microbial phenotype. For example, a study showed that differences in saccharolytic/proteolytic fermentation ratio may depend on microbial enterotype 
(microbial community stratification in Bacteroides, Prevotella, Ruminococcus) ${ }^{42}$. For instance, the Bacteroides enterotype has been linked to higher consumption of protein and saturated fats, whereas Prevotella enterotype has been associated to a lower protein intake ${ }^{43}$.

In conclusion, the present data do not show any alteration in proteolytic fermentation metabolites (fecal BCFA) and the saccharolytic metabolites lactate, succinate, valerate and caproate with degree of insulin resistance. Future nutritional interventions with the aim to modulate saccharolytic/proteolytic fermentation should measure the kinetics of these metabolites as well as plasma concentrations taking into account the metabolic ${ }^{44-46}$ and microbial phenotypes ${ }^{42}$ 47,48 . 


\section{References}

1. Canfora EE, Meex RCR, Venema K, Blaak EE. Gut microbial metabolites in obesity, NAFLD and T2DM. Nat Rev Endocrinol. 2019. Epub 2019/01/24. doi: 10.1038/s41574-019-0156-z. PubMed PMID: 30670819.

2. Canfora EE, Jocken JW, Blaak EE. Short-chain fatty acids in control of body weight and insulin sensitivity. Nat Rev Endocrinol. 2015;11(10):577-91. doi: 10.1038/nrendo.2015.128.

3. Koliada A, Syzenko G, Moseiko V, Budovska L, Puchkov K, Perederiy V, et al. Association between body mass index and Firmicutes/Bacteroidetes ratio in an adult Ukrainian population. BMC Microbiol. 2017;17(1):120-. doi: 10.1186/s12866017-1027-1. PubMed PMID: 28532414.

4. Turnbaugh PJ, Ley RE, Mahowald MA, Magrini V, Mardis ER, Gordon JI. An obesity-associated gut microbiome with increased capacity for energy harvest. Nature. 2006;444(7122):1027-31. doi: 10.1038/nature05414.

5. Ley RE, Bäckhed F, Turnbaugh P, Lozupone CA, Knight RD, Gordon JI. Obesity alters gut microbial ecology. Proceedings of the National Academy of Sciences of the United States of America. 2005;102(31):11070. doi: 10.1073/pnas.0504978102.

6. Castaner O, Goday A, Park Y-M, Lee S-H, Magkos F, Shiow S-ATE, et al. The Gut Microbiome Profile in Obesity: A Systematic Review. Int J Endocrinol. 2018;2018:4095789-. doi: 10.1155/2018/4095789. PubMed PMID: 29849617.

7. Mariat D, Firmesse O, Levenez F, Guimarăes VD, Sokol H, Doré J, et al. The Firmicutes/Bacteroidetes ratio of the human microbiota changes with age. BMC Microbiol. 2009;9(1):123. doi: 10.1186/1471-2180-9-123.

8. Schwiertz A, Taras D, Schäfer K, Beijer S, Bos NA, Donus C, et al. Microbiota and SCFA in Lean and Overweight Healthy Subjects. Obesity. 2010;18(1):190-5. doi: 10.1038/oby.2009.167.

9. Shen J, Obin MS, Zhao L. The gut microbiota, obesity and insulin resistance. Molecular Aspects of Medicine. 2013;34(1):39-58. doi: 10.1016/j.mam.2012.11.001.

10. Koh A, De Vadder F, Kovatcheva-Datchary P, Bäckhed F. From Dietary Fiber to Host Physiology: Short-Chain Fatty Acids as Key Bacterial Metabolites. Cell. 2016;165(6):1332-45. doi: 10.1016/j.cell.2016.05.041.

11. Mika A, Stepnowski P, Kaska L, Proczko M, Wisniewski P, Sledzinski M, et al. A comprehensive study of serum odd- and branched-chain fatty acids in patients with excess weight. Obesity (Silver Spring). 2016;24(8):1669-76. Epub 2016/06/30. doi: 10.1002/oby.21560. PubMed PMID: 27355152.

12. Ran-Ressler RR, Bae S, Lawrence P, Wang DH, Brenna JT. Branched-chain fatty acid content of foods and estimated intake in the USA. Br J Nutr. 2014;112(4):565-72. Epub 2014/05/17. doi: 10.1017/s0007114514001081. PubMed PMID: 24830474; PubMed Central PMCID: PMCPMC4381348. 
13. Blachier F, Beaumont M, Portune KJ, Steuer N, Lan A, Audebert M, et al. High-protein diets for weight management: Interactions with the intestinal microbiota and consequences for gut health. A position paper by the my new gut study group. Clinical Nutrition. 2019;38(3):1012-22. doi: 10.1016/j.clnu.2018.09.016. 14. Tiihonen K, Ouwehand AC, Rautonen N. Effect of overweight on gastrointestinal microbiology and immunology: correlation with blood biomarkers. British Journal of Nutrition. 2010;103(7):1070-8. Epub 2009/11/24. doi: 10.1017/S0007114509992807.

15. Granado-Serrano AB, Martín-Garí M, Sánchez V, Riart Solans M, Berdún R, Ludwig IA, et al. Faecal bacterial and short-chain fatty acids signature in hypercholesterolemia. Scientific Reports. 2019;9(1):1772. doi: 10.1038/s41598-01938874-3.

16. Da Silva HE, Teterina A, Comelli EM, Taibi A, Arendt BM, Fischer SE, et al. Nonalcoholic fatty liver disease is associated with dysbiosis independent of body mass index and insulin resistance. Sci Rep. 2018;8(1):1466. Epub 2018/01/25. doi: 10.1038/s41598-018-19753-9. PubMed PMID: 29362454; PubMed Central PMCID: PMCPMC5780381.

17. Ridaura VK, Faith JJ, Rey FE, Cheng J, Duncan AE, Kau AL, et al. Gut Microbiota from Twins Discordant for Obesity Modulate Metabolism in Mice. Science. 2013;341(6150):1241214. doi: 10.1126/science.1241214.

18. Vogelzangs N, van der Kallen CJH, van Greevenbroek MMJ, van der Kolk BW, Jocken JWE, Goossens GH, et al. Metabolic profiling of tissue-specific insulin resistance in human obesity: results from the Diogenes study and the Maastricht Study. International Journal of Obesity. 2020. doi: 10.1038/s41366-020-0565-z.

19. Gannon NP, Schnuck JK, Vaughan RA. BCAA Metabolism and Insulin Sensitivity - Dysregulated by Metabolic Status? Mol Nutr Food Res. 2018;62(6):e1700756. Epub 2018/01/30. doi: 10.1002/mnfr.201700756. PubMed PMID: 29377510.

20. Newgard CB, An J, Bain JR, Muehlbauer MJ, Stevens RD, Lien LF, et al. A branched-chain amino acid-related metabolic signature that differentiates obese and lean humans and contributes to insulin resistance. Cell metabolism. 2009;9(4):311-26. Epub 2009/04/10. doi: 10.1016/j.cmet.2009.02.002. PubMed PMID: 19356713; PubMed Central PMCID: PMCPMC3640280.

21. van der Beek CM, Canfora EE, Kip AM, Gorissen SHM, Olde Damink SWM, van Eijk HM, et al. The prebiotic inulin improves substrate metabolism and promotes short-chain fatty acid production in overweight to obese men. Metabolism. 2018;87:25-35. Epub 2018/06/29. doi: 10.1016/j.metabol.2018.06.009. PubMed PMID: 29953876.

22. Müller M, Hermes G, Canfora E, Holst J, Zoetendal E, Smidt H, et al. Effect of wheat bran derived prebiotic supplementation on gastrointestinal transit, gut microbiota, and metabolic health: a randomized controlled trial in healthy adults with a slow gut transit. Gut Microbes. 2020. doi: 10.1080/19490976.2019.1704141. 
23. NIH. National Institutes of Health. 2019. PubMed PMID: Clinical trial No. NCT03714646.

24. Canfora EE, Blaak EE. Beta Glucan and Acetate production. Clinicaltrialsgov. 2018. PubMed PMID: NCT03714646.

25. Frayn KN. Calculation of substrate oxidation rates in vivo from gaseous exchange. J Appl Physiol Respir Environ Exerc Physiol. 1983;55(2):628-34. Epub 1983/08/01. doi: 10.1152/jappl.1983.55.2.628. PubMed PMID: 6618956.

26. Weir JBdV. New methods for calculating metabolic rate with special reference to protein metabolism. J Physiol. 1949;109(1-2):1-9. PubMed PMID: PMC1392602.

27. Garcia-Villalba R, Gimenez-Bastida JA, Garcia-Conesa MT, TomasBarberan FA, Carlos Espin J, Larrosa M. Alternative method for gas chromatography-mass spectrometry analysis of short-chain fatty acids in faecal samples. J Sep Sci. 2012;35(15):1906-13. Epub 2012/08/07. doi: 10.1002/jssc.201101121. PubMed PMID: 22865755.

28. Matthews DR, Hosker JP, Rudenski AS, Naylor BA, Treacher DF, Turner RC. Homeostasis model assessment: insulin resistance and beta-cell function from fasting plasma glucose and insulin concentrations in man. Diabetologia. 1985;28(7):412-9. Epub 1985/07/01. doi: 10.1007/bf00280883. PubMed PMID: 3899825.

29. Fernandes J, Su W, Rahat-Rozenbloom S, Wolever TMS, Comelli EM. Adiposity, gut microbiota and faecal short chain fatty acids are linked in adult humans. Nutrition \& diabetes. 2014;4(6):e121-e. doi: 10.1038/nutd.2014.23. PubMed PMID: 24979150.

30. Le Roy $\mathrm{T}$, Llopis M, Lepage P, Bruneau A, Rabot S, Bevilacqua C, et al. Intestinal microbiota determines development of non-alcoholic fatty liver disease in mice. Gut. 2013;62(12):1787-94. Epub 2012/12/01. doi: 10.1136/gutjnl-2012303816. PubMed PMID: 23197411.

31. Feng W, Ao H, Peng C. Gut Microbiota, Short-Chain Fatty Acids, and Herbal Medicines. Frontiers in Pharmacology. 2018;9(1354). doi: 10.3389/fphar.2018.01354.

32. Müller M, Canfora EE, Blaak EE. Gastrointestinal Transit Time, Glucose Homeostasis and Metabolic Health: Modulation by Dietary Fibers. Nutrients. 2018;10(3):275. doi: 10.3390/nu10030275. PubMed PMID: 29495569.

33. den Besten G, van Eunen K, Groen AK, Venema K, Reijngoud D-J, Bakker $\mathrm{BM}$. The role of short-chain fatty acids in the interplay between diet, gut microbiota, and host energy metabolism. J Lipid Res. 2013;54(9):2325-40. Epub 2013/07/02. doi: 10.1194/jlr.R036012. PubMed PMID: 23821742.

34. Tang Z-Z, Chen G, Hong Q, Huang S, Smith HM, Shah RD, et al. MultiOmic Analysis of the Microbiome and Metabolome in Healthy Subjects Reveals Microbiome-Dependent Relationships Between Diet and Metabolites. Frontiers in Genetics. 2019;10(454). doi: 10.3389/fgene.2019.00454. 
35. Nagpal R, Mainali R, Ahmadi S, Wang S, Singh R, Kavanagh K, et al. Gut microbiome and aging: Physiological and mechanistic insights. Nutr Healthy Aging. 2018;4(4):267-85. doi: 10.3233/NHA-170030. PubMed PMID: 29951588.

36. Zhong H, Penders J, Shi Z, Ren H, Cai K, Fang C, et al. Impact of early events and lifestyle on the gut microbiota and metabolic phenotypes in young school-age children. Microbiome. 2019;7(1):2. doi: 10.1186/s40168-018-0608-z.

37. Müller M, Hernández MAG, Goossens GH, Reijnders D, Holst JJ, Jocken JWE, et al. Circulating but not faecal short-chain fatty acids are related to insulin sensitivity, lipolysis and GLP-1 concentrations in humans. Scientific Reports. 2019;9(1):12515. doi: 10.1038/s41598-019-48775-0.

38. Aragonès G, Colom-Pellicer M, Aguilar C, Guiu-Jurado E, Martínez S, Sabench F, et al. Circulating microbiota-derived metabolites: a "liquid biopsy? International Journal of Obesity. 2020;44(4):875-85. doi: 10.1038/s41366-019-0430-0. 39. Heimann E, Nyman M, Pålbrink A-K, Lindkvist-Petersson K, Degerman E. Branched short-chain fatty acids modulate glucose and lipid metabolism in primary adipocytes. Adipocyte. 2016;5(4):359-68. doi: 10.1080/21623945.2016.1252011. PubMed PMID: 27994949.

40. Russell WR, Gratz SW, Duncan SH, Holtrop G, Ince J, Scobbie L, et al. Highprotein, reduced-carbohydrate weight-loss diets promote metabolite profiles likely to be detrimental to colonic health. The American Journal of Clinical Nutrition. 2011;93(5):1062-72. doi: 10.3945/ajcn.110.002188.

41. Hald S, Schioldan AG, Moore ME, Dige A, Lærke HN, Agnholt J, et al. Effects of Arabinoxylan and Resistant Starch on Intestinal Microbiota and ShortChain Fatty Acids in Subjects with Metabolic Syndrome: A Randomised Crossover Study. PLoS One. 2016;11(7):e0159223. Epub 2016/07/21. doi: 10.1371/journal.pone.0159223. PubMed PMID: 27434092; PubMed Central PMCID: PMCPMC4951149.

42. Arumugam M, Raes J, Pelletier E, Le Paslier D, Yamada T, Mende DR, et al. Enterotypes of the human gut microbiome. Nature. 2011;473(7346):174-80. doi: 10.1038/nature09944.

43. Wu GD, Chen J, Hoffmann C, Bittinger K, Chen Y-Y, Keilbaugh SA, et al. Linking Long-Term Dietary Patterns with Gut Microbial Enterotypes. Science. 2011;334(6052):105. doi: 10.1126/science.1208344.

44. Hansen AMB, Wium C, Lee S, Tierney AC, McCarthy D, Roche HM, et al. Substantial inter-individual variations in insulin secretion and sensitivity across the glucometabolic spectrum. Scandinavian Journal of Clinical and Laboratory Investigation. 2020:1-9. doi: 10.1080/00365513.2020.1730433.

45. Bouter K, Bakker GJ, Levin E, Hartstra AV, Kootte RS, Udayappan SD, et al. Differential metabolic effects of oral butyrate treatment in lean versus metabolic syndrome subjects. Clin Transl Gastroenterol. 2018;9(5):155-. doi: 10.1038/s41424018-0025-4. PubMed PMID: 29799027.

46. Lim J, Henry CJ, Haldar S. Vinegar as a functional ingredient to improve postprandial glycemic control-human intervention findings and molecular 
mechanisms. Mol Nutr Food Res. 2016;60(8):1837-49. Epub 2016/05/24. doi: 10.1002/mnfr.201600121. PubMed PMID: 27213723.

47. Kovatcheva-Datchary P, Nilsson A, Akrami R, Lee YS, De Vadder F, Arora $\mathrm{T}$, et al. Dietary Fiber-Induced Improvement in Glucose Metabolism Is Associated with Increased Abundance of Prevotella. Cell metabolism. 2015;22(6):971-82. Epub 2015/11/11. doi: 10.1016/j.cmet.2015.10.001. PubMed PMID: 26552345.

48. Holscher HD. Dietary fiber and prebiotics and the gastrointestinal microbiota. Gut Microbes. 2017;8(2):172-84. Epub 2017/02/06. doi: 10.1080/19490976.2017.1290756. PubMed PMID: 28165863. 


\section{CHAPTER 6}

Acetate does not affect palmitate oxidation and AMPK phosphorylation in human primary skeletal muscle cells

Running title: Acetate and fat oxidation in human muscle cells

Manuel A. González Hernández, Ellen E. Blaak, Nicole T. H. Hoebers, Yvonne P. G. Essers, Emanuel E. Canforat and Johan W. E. Jockent

† shared last authors

To be submitted 


\begin{abstract}
Our recent in vivo human studies showed that colonic administration of sodium acetate (SA) resulted in increased circulating acetate levels, which was accompanied by increments in whole-body fat oxidation in overweight-obese men. Since skeletal muscle has a major role in whole-body fat oxidation, we aimed to investigate effects of SA on fat oxidation and underlying mechanisms in human primary skeletal muscle cells (HSkMC). We investigated the dose (0-5 mmol/l) and time $(1,4,20$ and $24 \mathrm{~h})$ effect of SA on complete and incomplete endogenous and exogenous oxidation of ${ }^{14} \mathrm{C}$-labeled palmitate in HSkMC derived from a lean insulin sensitive male donor. Both physiological $(0.1$ and $0.25 \mathrm{mmol} / \mathrm{l})$ and supraphysiological $(0.5,1$ and $5 \mathrm{mmol} / \mathrm{l})$ concentrations of SA neither increased endogenous nor exogenous fat oxidation over time in HSkMC. In addition, no effect of SA was observed on $T h r^{172}-A M P K \alpha$ phosphorylation. In conclusion, our previously observed in vivo effects of SA on whole-body fat oxidation in men may not be explained via direct effects on HSkMC fat oxidation. Nevertheless, SAmediated effects on whole-body fat oxidation may be triggered by other mechanisms including gut-derived hormones or may occur in other metabolically active tissues.
\end{abstract}




\section{Introduction}

In the last decades obesity has reached pandemic proportions worldwide ${ }^{1}$, indicating the necessity to take action and prevent the development of obesityrelated comorbidities. Obesity is characterized by an imbalance in energy intake and expenditure, with the excess energy stored in adipose tissue depots. It is increasingly clear that not adipose mass per se but rather adipose tissue dysfunction plays a central role in the observed metabolic derangements ${ }^{1}$. A limited buffering capacity may result in systemic lipid overflow and an increased lipid supply to non-adipose tissues. Consequently, this may, together with an impaired oxidative capacity cause ectopic fat deposition in important metabolically active tissues (i.e. skeletal muscle, liver), which may link to the development of insulin resistance ${ }^{2}$.

Of note, evidence is accumulating that energy and substrate metabolism and insulin sensitivity may be modulated by gut microbially-derived metabolites, thus bringing attention to the cross-talk between gut-microbiota-derived metabolites and host metabolic health ${ }^{3,4}$. In the colon, fermentation of indigestible food compounds by the resident microbiota plays a pivotal role in the production of short-chain fatty acids (SCFA), including acetate, propionate and butyrate ${ }^{3,5,6}$. Of these SCFA, acetate is the most abundant in the colon and has potential beneficial effects on energy expenditure and substrate metabolism ${ }^{7}$. Circulating levels of acetate can reach physiological concentrations that range between 5 up to an average of $450 \mu \mathrm{mol} / \mathrm{l}$, predominantly after prebiotic consumption ${ }^{4,8-15}$. Subsequently, gut-derived acetate seems to have a broad metabolic role, since the SCFA-sensing G protein-coupled receptors (GPR, 41/43) are expressed in various tissues such as the adipose tissue ${ }^{16}$, skeletal muscle, liver ${ }^{17}$ and pancreatic beta cells ${ }^{18,19}$. Circulating acetate has been shown to have antilipolytic effects in adipose tissue in in vitro ${ }^{20-22}$, animal ${ }^{20,23}$ and human studies ${ }^{24-27}$, thus potentially reducing lipid overflow, improving lipid buffering capacity and impacting peripheral insulin sensitivity. Other potential metabolic effects may include the induction of satiety mediated via the stimulation of glucagon-like peptide 1 (GLP-1) and peptide YY (PYY) secretion ${ }^{28,29}$.

In addition to satiety, SCFA effects on intestinal gluconeogenesis and increased skeletal muscle fat oxidation have been observed at least in rodents ${ }^{3}$. Furthermore, an in vitro study in L6 myotubes showed that acetate acutely (following $2 \mathrm{~min}$ ) increased AMP-activated protein kinase (AMPK) phosphorylation via increased adenosine monophosphate (AMP)/adenosine triphosphate (ATP) ratio ${ }^{30}$. Moreover, intragastric injection of acetate in mice showed a rapid increase in muscle AMP/ATP ratio and AMPK phosphorylation ${ }^{31}$. In addition to effects on skeletal muscle metabolism, acetate mediated upregulation of genes involved in fat oxidation (uncoupling protein 2, peroxisome proliferator-activated receptor alpha and carnitine palmitoyl transferase 1) and increased $A M P K$ phosphorylation in murine liver ${ }^{32-34}$. 
Although no actual substrate oxidation was measured, these studies suggest that acetate may increase liver and skeletal muscle oxidative capacity in an AMPK-mediated fashion, at least in rodent models. Our recently published human intervention study in overweight/obese normoglycemic men showed that acute distal, not proximal, colonic acetate infusions $(180 \mathrm{mmol} / \mathrm{l})$ increased fasting fat oxidation by around $25 \%$, which may be possibly mediated via increased circulating acetate levels ${ }^{7}$. Together, these data suggest that the observed in vivo effects on whole-body fat oxidation in overweight/obese humans may be partly mediated via a direct effect of circulating acetate on skeletal muscle fat oxidation. Therefore, we hypothesized that SA (sodium acetate) increases fat oxidation in a time- and dose-dependent manner in differentiated human primary muscle cells (HSkMC).

Fat oxidation was investigated by measuring SA effects on both endogenous (intracellular) and exogenous (extracellular) ${ }^{14} \mathrm{C}$-palmitate oxidation. Additionally, from a mechanistic perspective, we investigated SA-mediated effects on AMPK phosphorylation in HSkMC. 


\section{Materials and methods}

HSkMC were isolated from rectus abdominal muscle tissue following surgery as described previously from a healthy male donor, insulin sensitive (HOMA-IR=0.37), 45 year old with a BMI of $23.5 \mathrm{~kg} / \mathrm{m}^{235}$. Cells were proliferated using Dulbecco's modified eagle medium (DMEM) low glucose $(5.5 \mathrm{mmol} / \mathrm{l})$ (\#D6046-500ml, Sigma-Aldrich) supplemented with 16\% fetal bovine serum (FBS) (Batch BDC-11933, Bodinco BV), 0.05\% bovine serum albumin (BSA) (\#A4503100g, Sigma-Aldrich), $1 \mu \mathrm{mol} / \mathrm{l}$ dexamethasone (\#D4902-100mg, Sigma-Aldrich), $0.5 \mathrm{mg} / \mathrm{ml}$ bovine fetuin (\#10505053, Thermo Fisher Scientific), 1x antibiotic/antimycotic (\#15240-062, Thermo Fisher Scientific) and $0.01 \mu \mathrm{g} / \mathrm{ml}$ recombinant human epidermal growth factor (\#PHG0311, Thermo Fisher Scientific). Cells were cultured in differentiation medium containing MEM-Alpha medium/ Glutamax (\#32561-029, Thermo Fisher Scientific) supplemented with 2\% FBS, $0.5 \mathrm{mg} / \mathrm{ml}$ bovine fetuin and $1 \mathrm{x}$ antibiotic/antimycotic. Antibodies against total AMPK $\alpha$, and phosphorylated $T h r^{172}-A M P K \alpha$ were purchased from Cell Signaling (\#2603 and \#2535, respectively MA, USA). Secondary antibodies (Goatanti-Rabbit HRP) were purchased from Vector Labs (\#1000). SA was purchased from Sigma-Aldrich (\#S2889). For fat oxidation assay, we used 4-(2-Hydroxyethyl) piperazine-1-ethanesulfonic acid (HEPES, \#H3375, Sigma-Aldrich), L-carnitine hydrochloric acid (carnitine, \#C0283, Sigma-Aldrich), ${ }^{14} \mathrm{C}$-palmitate (\#NEC075H250uC, Perkin Elmer), non-labelled palmitate (\#P0500, Sigma-Aldrich) and perchloric acid (\#244252, Sigma-Aldrich).

\section{Cell culture}

HSkMC were cultivated in proliferation medium under $5 \% \mathrm{CO}_{2}$ at $37^{\circ} \mathrm{C}$. For differentiation towards functional myotubes, medium was changed to differentiation medium when reaching $80 \%$ confluence, as described above. At day 8-11 of differentiation viable myotubes were used for fat oxidation experiments

\section{Cytotoxicity assay}

A fluorometric lactate dehydrogenase (LDH) assay was used for estimating the number of nonviable cells, as marker of cytotoxicity (\#G7890, Promega). Differentiated cells were cultured without $(0 \mathrm{mmol} / \mathrm{l})$, or with physiological $(0.1$ $\mathrm{mmol} / \mathrm{l})$ and supraphysiological (1 and $5 \mathrm{mmol} / \mathrm{l})$ concentrations of SA during 4 and 24 hours. Fluorescence was measured using a Spectramax plate reader (Molecular Devices, California, USA). Percentage of cytotoxicity was calculated in comparison to Triton X-100 (100\% lysis positive control) with the same incubation times. 


\section{Fat oxidation}

For measurement of in vitro fat oxidation cells were incubated with ${ }^{14} \mathrm{C}$ labelled palmitate $(250 \mu \mathrm{Ci} / \mathrm{ml}$; PerkinElmer, Boston, MA) and non-labeled palmitate. Palmitate was coupled to fatty acid free BSA (7.5\%) together with HEPES (100 mmol/l) and carnitine $(40 \mathrm{mmol} / \mathrm{l})$. After SA incubation, complete $\left({ }^{14} \mathrm{CO}_{2}\right)$ and incomplete $\left({ }^{14} \mathrm{ASM}\right.$, acid soluble metabolites) oxidation products were measured, as previously described ${ }^{36}$.

${ }^{14} \mathrm{ASM}$ include acetyl-CoA, acetyl carnitines, ketone bodies and TCA cycle intermediaries. Briefly, following incubation, medium was transferred to a custom-made Teflon 24-well $\mathrm{CO}_{2}$ trapping plate that was sealed, 70\% perchloric acid was injected (Hamilton syringe, 1705N) through a silicon layer in the lid directly into the media. This moved the $\mathrm{CO}_{2}$ through a tunnel to an adjacent well where it was trapped in $1 \mathrm{~N} \mathrm{NaOH}$. After overnight trapping, complete and incomplete oxidation products were measured by Scintillation counting (using a Tri-Carb 2910 TR liquid scintillation analyzer, Perkin Elmer). For measuring endogenous fat oxidation, cells were pre-incubated for 24 hours with ${ }^{14} \mathrm{C}$ - labelled palmitate $(250 \mu \mathrm{Ci} / \mathrm{ml}$; PerkinElmer, Boston, MA) without carnitine and washed prior to SA incubations.

\section{Western blotting}

Following incubations without $(0 \mathrm{mmol} / \mathrm{l})$ or with SA $(0.1$ or $0.5 \mathrm{mmol} / \mathrm{l})$, cells were washed with ice-cold PBS and lysed with RIPA buffer supplemented with protease (\#04693132001, Sigma-Aldrich) and phosphatase (\#04906845001, Sigma-Aldrich) inhibitors. Samples were vortexed, left on ice ( 10 min) and snapfrozen in liquid nitrogen. Supernatants were subjected to western blot analysis. Briefly, a total of $10 \mu \mathrm{g}$ of protein was loaded on a 4-12\% SDS-PAGE gel using a Criterion XT precast gel (10-300 kilodaltons, Bio-Rad) with MOPS buffer (\#1610788, Biorad). After separation, proteins were transferred using a Criterion blot system (Bio-Rad) in 1xTG buffer (20\% methanol + 0.05\% SDS) onto a $0.45 \mu \mathrm{m}$ nitrocellulose membrane (GE Healthcare, Netherlands), and membranes were blocked using tris-buffered saline (TBS)/3\%BSA/0.1\%Tween-20 (for phosphorylated antibodies and for non-phosphorylated antibodies they were blocked in TBS/5\%non-fat dry milk (NFDM)/0.1\%Tween-20). Next, the membrane was incubated with primary antibodies (rabbit-anti human p-AMPK diluted $1: 1000$ in $\mathrm{TBS} / 3 \% \mathrm{BSA} / 0.1 \%$ Tween and rabbit-anti human AMPK in $\mathrm{TBS} / 5 \% / \mathrm{NFDM} / 0.1 \%$ Tween-20). After overnight incubations at $4^{\circ} \mathrm{C}$, membranes were washed three times with tris-buffered saline tween (TBST) and incubated with HRP-conjugated secondary antibodies for $1 \mathrm{~h}$ (goat-anti-rabbit HRP) diluted 1:10,000 in TBS 3\%BSA/0.1\%Tween-20 or in TBS/5\%NFDM/0.1\%Tween-20).

Finally, after washing with TBST, last time with TBS, bands were visualized using ECL substrate (Super Signal West Femto, \#34095, Thermo Scientific) according to the protocol supplied by the manufacturer using the Bio-Rad ChemiDoc imaging system (Biorad). 


\section{Statistical analysis}

Values are expressed as mean and standard deviation. Significance was determined using the nonparametric Mann Whitney U-test when comparing two groups or the Kruskal-Wallis H-test, for multiple comparisons. In case of significant Kruskal-Wallis, Dunn's post hoc test was performed.

Statistics were performed using the GraphPad Prism 5.0a software package (GraphPad Software, San Diego, CA, USA) and a P<0.05 (two-sided P-value) was considered statistically significant. 


\section{Results}

\section{Time-dependent effect of SA on exogenous and endogenous palmitate}

oxidation

First of all, both acute (4h) as well as chronic (24h) incubation with physiological $(0.1 \mathrm{mmol} / \mathrm{l})$ and supraphysiological concentrations $(1$ and $5 \mathrm{mmol} / \mathrm{l})$ of SA did not affect LDH activity, indicating no cytotoxic effect of SA in HSkMC (see S1 Fig). Secondly, we investigated whether SA has a time-dependent effect on complete or incomplete exogenous/endogenous ${ }^{14} \mathrm{C}$-palmitate oxidation in differentiated HSkMC. Briefly, cells were incubated with a physiological concentration of SA $(0.1 \mathrm{mmol} / \mathrm{l})$ or without $(0 \mathrm{mmol} / \mathrm{l})$ for up to 24 hours. As expected, and shown in Fig 1, an increase in fat oxidation was observed over time. However, both complete (Fig 1A) and incomplete (Fig 1B) exogenous fat oxidation were not affected when comparing SA to control treated cells after acute (1-4h) and chronic (20-24h) exposure. Next, to investigate the effect of SA on endogenous fat oxidation cells were first pre-incubated with ${ }^{14} \mathrm{C}$-palmitate for $24 \mathrm{~h}$ and after removal of the label, cells were incubated with SA $(0.1 \mathrm{mmol} / \mathrm{l})$ or without $(0$ $\mathrm{mmol} / \mathrm{l}$ ) for up to 24 hours. Although we found a significant increase over time, no differences were observed between SA and control treated cells in complete (Fig 1C) and incomplete (Fig 1D) endogenous fat oxidation at each timepoint.
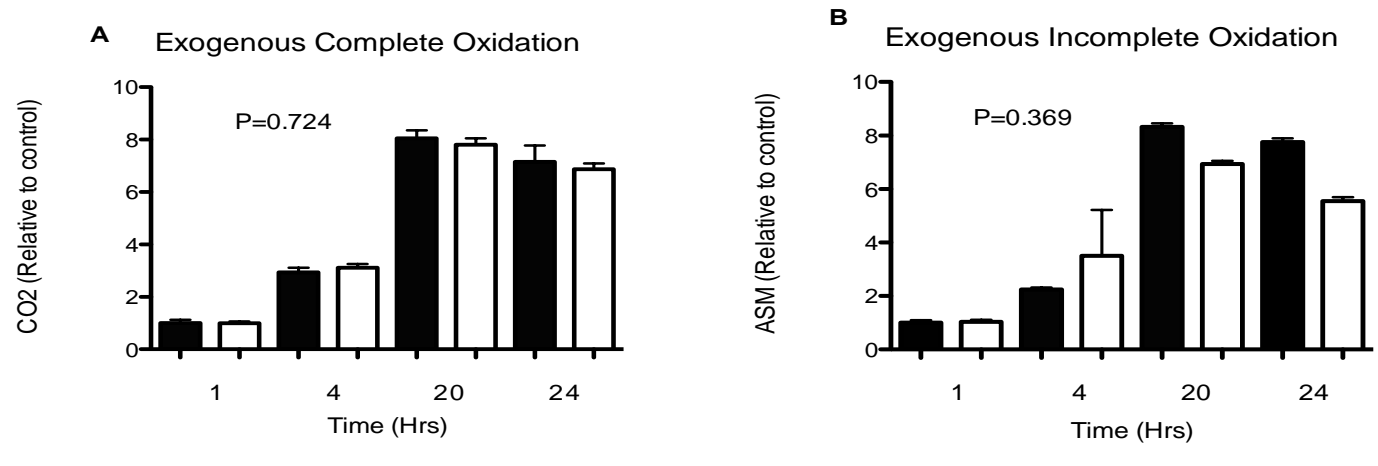

C Endogenous Complete Oxidation

D Endogenous Incomplete Oxidation
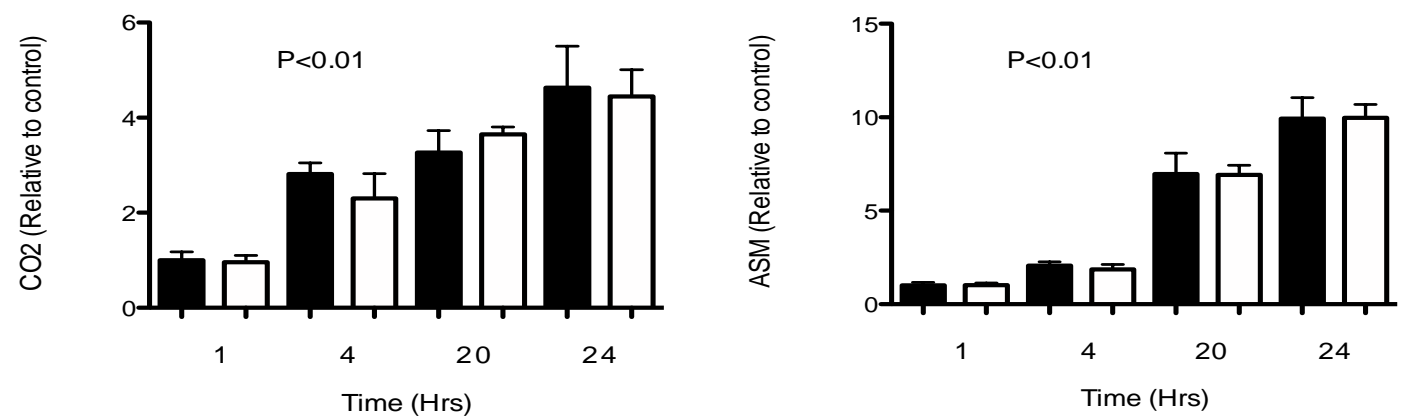

Figure 1 Time-effect of SA on exogenous and endogenous fat oxidation in HSkMC. (A) Complete $\left({ }^{14} \mathrm{CO}_{2}\right)$ and (B) incomplete (ASM) ${ }^{14} \mathrm{C}$-palmitate exogenous oxidation (2 independent experiments) was measured following 1, 4, 20 and 24 hours of incubation without $(0 \mathrm{mmol} / \mathrm{l})$ or with $(0.1 \mathrm{mmol} / \mathrm{l}) \mathrm{SA}$. In addition, following 24h pre-incubation with ${ }^{14} \mathrm{C}$-palmitate, (C) complete $\left({ }^{14} \mathrm{CO}_{2}\right)$ and (D) incomplete (ASM) ${ }^{14} \mathrm{C}$-palmitate endogenous oxidation ( 2 independent experiments) was measured following 1, 4, 20- and 24-hours incubation without $(0 \mathrm{mmol} / \mathrm{l})$ or with $(0.1 \mathrm{mmol} / \mathrm{l}) \mathrm{SA}$. Data expressed as mean and standard deviation and expressed as relative to control at $\mathrm{T}=0$ (black). $\mathrm{P}$ value corresponds to Kruskal-Wallis test. Post-hoc test showed no differences between control and acetate treated cells at each timepoint. 


\section{Dose-dependent effect of SA on exogenous or endogenous fat oxidation}

Subsequently, we investigated the dose-effect of SA on exogenous fat oxidation in differentiated HSkMC. Cells were incubated without $(0 \mathrm{mmol} / \mathrm{l})$, or with physiological $(0.1,0.25,0.5 \mathrm{mmol} / \mathrm{l})$ and supraphysiological ( 1 and $5 \mathrm{mmol} / \mathrm{l})$ concentrations of SA for up to 20h. However, as shown in Fig 2, SA did neither increase complete (Fig 2A) nor incomplete (Fig 2B) exogenous fat oxidation as compared to control treated cells.

To investigate dose-dependent effect of SA on endogenous fat oxidation cells were first pre-incubated with ${ }^{14} \mathrm{C}$-palmitate for $24 \mathrm{~h}$ and after removal of the label, cells were treated without $(0 \mathrm{mmol} / \mathrm{l})$, or with physiological $(0.1,0.25,0.5$ $\mathrm{mmol} / \mathrm{l}$ ) and supraphysiological (1 and $5 \mathrm{mmol} / \mathrm{l}$ ) concentrations of SA for up to 20h. In line with the exogenous oxidation data, we did not observe a dosedependent increase in complete (Fig 2C) palmitate oxidation as compared to control treated cells. However, we found a significant decrease in the incomplete oxidation (Fig 2D), but only following incubation with the highest dose $(5 \mathrm{mmol} / \mathrm{l})$ of SA $(\mathrm{P}<0.05)$.

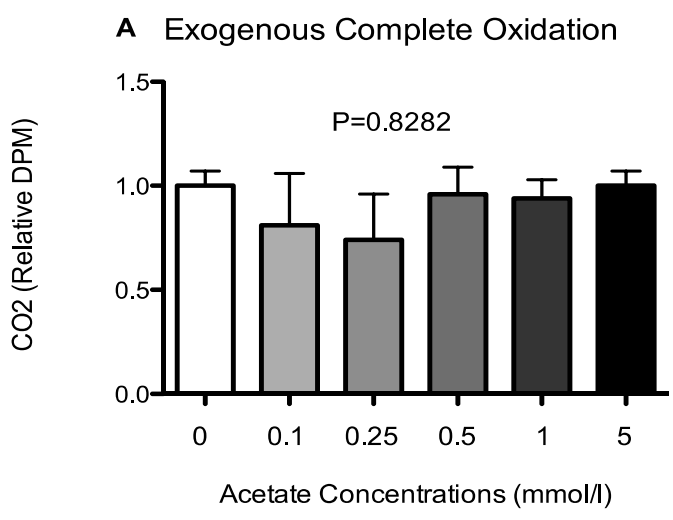

C Endogenous Complete Oxidation

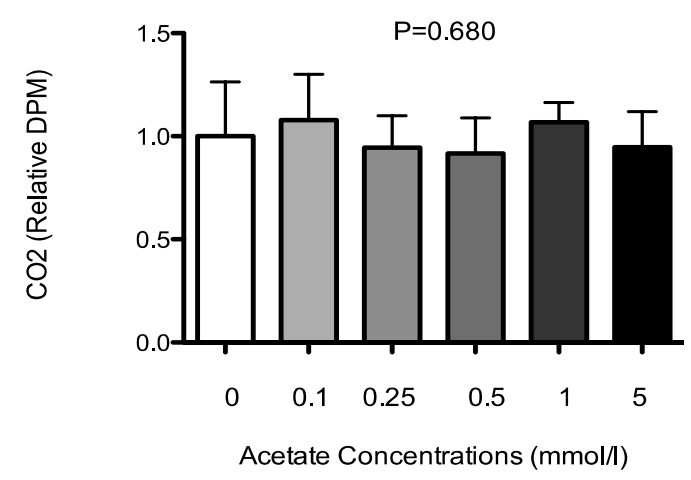

B Exogenous Incomplete Oxidation

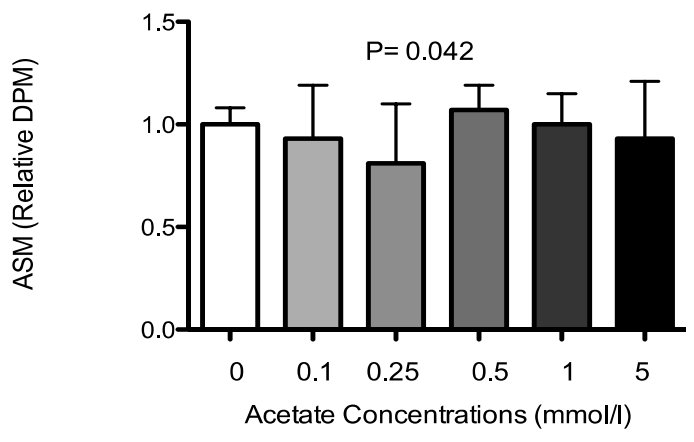

D Endogenous Incomplete Oxidation

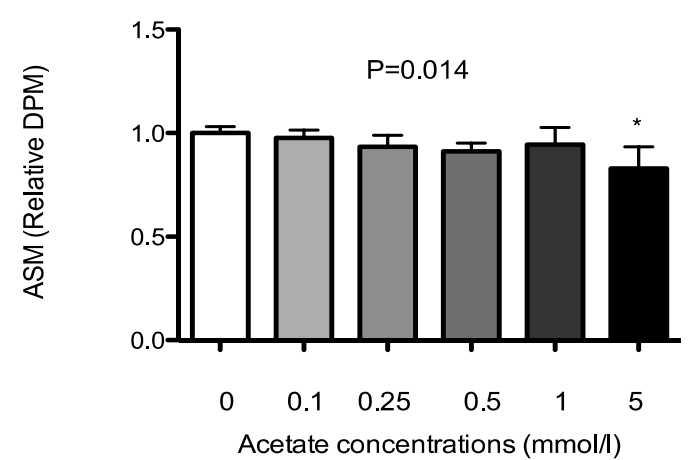

Figure 2. Dose-effect of SA on exogenous and endogenous fat oxidation in HSkMC. (A) Complete $\left({ }^{14} \mathrm{CO}_{2}\right)$ and (B) incomplete (ASM) ${ }^{14} \mathrm{C}$-palmitate exogenous oxidation (5 independent experiments) was measured following $20 \mathrm{~h}$ incubation without $(0 \mathrm{mmol} / \mathrm{l})$ or with $(0.1-5 \mathrm{mmol} / \mathrm{l}) \mathrm{SA}$. In addition, following $24 \mathrm{~h}$ preincubation with ${ }^{14} \mathrm{C}$-palmitate, (C) complete $\left({ }^{14} \mathrm{CO}_{2}\right)$ and (D) incomplete (ASM) ${ }^{14} \mathrm{C}$-palmitate endogenous oxidation (4 independent experiments) was measured following $20 \mathrm{~h}$ incubation without $(0 \mathrm{mmol} / \mathrm{l})$ or with (0.1-5 mmol/l) SA. Data expressed as mean and standard deviation and expressed as relative to control treated cells. DPM, disintegrations per minute. P value corresponds to Kruskal-Wallis test. Post-hoc test significance is compared to control $(0 \mathrm{mmol} / \mathrm{l})$ indicated as asterisk $\left({ }^{*}\right)$ when $\mathrm{P}<0.05$ 


\section{Effect of SA on AMPK phosphorylation}

In line with our oxidation data, we did not observe a time $(2 \mathrm{~min}, 10 \mathrm{~min}$ and 24h) or dose ( 0.1 and $0.5 \mathrm{mmol} / \mathrm{l})$ dependent effect on total or phosphorylated Thr ${ }^{172} A M P K \alpha$ content in HSkMC (Fig 3). Of interest, Thr ${ }^{172}-A M P K \alpha$ phosphorylation was increased in our HSkMC model following $30 \mathrm{~min}$ and 4-hour incubations with the major AMPK activator AICAR (2 mmol/l) (Fig S3). Nevertheless, this AICAR-mediated increase in AMPK phosphorylation was not accompanied by an increased complete nor incomplete exogenous/endogenous fat oxidation (Fig S2). In contrast, treatment of HSkMC using an inhibitor (etomoxir, $100 \mu \mathrm{mol} / \mathrm{l}$ ) showed marked changes in complete and incomplete palmitate oxidation, indicating that our HSkMC is dynamic and fully functional (Fig S2).

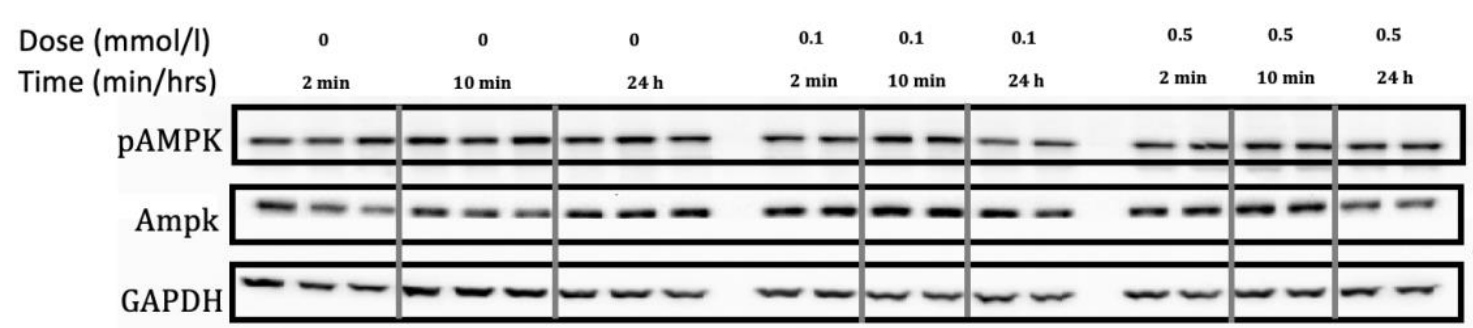

Figure 3. Time and dose-dependent effects of SA on total and pAMPK in HSkMC. Cells were incubated without $(0 \mathrm{mmol} / \mathrm{l})$ and with $(0.1$ and $0.5 \mathrm{mmol} / \mathrm{l}) \mathrm{SA}$ for 2 or $10 \mathrm{~min}$ and up to $24 \mathrm{~h}$. After incubation, cells were lysed with RIPA buffer supplemented with protease and phosphatase inhibitors and samples were subjected to western analysis for total $A M P K \alpha(62 \mathrm{kD}$ band), phosphorylated AMPK (Thr172-AMPK $\alpha$ ) and GAPDH (37kD band) was used as a loading control. 


\section{Discussion}

Recently we showed that distal (not proximal) colonic infusions of acetate and SCFA mixtures (rich in acetate) increased circulating acetate levels (120 min) in overweight/obese humans ${ }^{7,37}$. Of note, we found a positive relationship between increments in acetate levels and fasting whole-body fat oxidation. Therefore, we hypothesized that circulating acetate may directly affect fat oxidation in human skeletal muscle contributing to the observed increments in in vivo whole-body fat oxidation. To our knowledge, this is the first study that investigates the direct (dose/time) effects of SA on fat oxidation (endo-exogenous) and whether effects are dependent on AMPK phosphorylation in a HSkMC model. However, our data showed no time- or dose-dependent increase of SA on either endo- or exogenous palmitate oxidation in HSkMC. In line, AMPK phosphorylation was not affected by acute nor chronic SA treatment.

In vivo colonic infusions ${ }^{37,38}$ showed an acute effect on fasting whole-body fat oxidation in overweight-obese men with acetate concentrations reaching average plasma acetate levels of $40 \mu \mathrm{mol} / \mathrm{l}$. However, our in vitro experiments showed no dose- or time effect of SA, with physiological concentrations (0-0.5 $\mathrm{mmol} / \mathrm{l})$, reached after in vivo dietary fiber fermentation ${ }^{10-13}$, and no effect with supraphysiological concentrations (1-5 mmol/l). Although the actual interstitial/intracellular concentration of acetate in the skeletal muscle remains unknown, it has been suggested in rats ${ }^{39}$ and humans ${ }^{40}$ that acetate uptake in skeletal muscle is proportional to circulating levels. In support, using labelled ${ }^{13} \mathrm{C}$ SA in healthy lean individuals has shown ${ }^{13} \mathrm{CO}_{2}$ breath recovery rates between $40-$ $80 \%$, which may indicate acetate uptake and utilization/oxidation in the TCA cycle of metabolically relevant organs (i.e. liver, muscle) ${ }^{41-43}$. Of note, in our in vitro experiments SA was provided as a single bolus, and acetate may have been rapidly absorbed, and used into the tricarboxylic acid cycle (TCA) and saturate connecting metabolic pathways ${ }^{44}$. In support, acute intravenous infusions of acetate (2.5 mmol per min for $1 \mathrm{~h}$ ) in humans did not increase energy expenditure, which was partly explained by the fact that acetate might replace long chain fatty acids as preferred oxidation fuel in TCA-connected metabolic pathways ${ }^{45}$. Of interest, a slight time-dependent decrease in endogenous (incomplete) oxidation was observed (Fig 2D), which reached significance following incubation with the highest dose of SA (5 mmol/l) compared to control cells, possibly indicating preferential use of SA as oxidation fuel. Future studies using labeled SA are needed in order to investigate the metabolic fate of acetate in human skeletal muscle.

As mentioned above, our design (one single bolus of SA), may not mimic a continuous delivery of gut-derived acetate to the skeletal muscle. Acetate colonic absorption seems to be concentration dependent ${ }^{46}$ and to gradually increase plasma levels, thus reaching skeletal muscle in a more continuous manner in vivo. Nevertheless, our doses were based on human prebiotic interventions that report 
circulating acetate concentrations up to $450 \mu \mathrm{mol} / \mathrm{l}^{9-14}$. In support of a continuous supply, a 4-week supplementation of a high amount of fermentable (type 2) resistant starch $(30 \mathrm{~g} / \mathrm{d}$ vs rapidly digestible starch $20 \mathrm{~g})$ increased acetate uptake in skeletal muscle (and adipose tissue) in healthy subjects ${ }^{47}$. Although no fat oxidation was measured, the intervention improved peripheral insulin sensitivity (euglycemic hyperinsulinemic clamp) ${ }^{47}$, thus potentially indicating that a continuous supply of gut-derived acetate into the muscle (instead of a single bolus) is needed to induce major metabolic effects.

Based on previous animal data, acetate (oral injection) acutely increases phosphorylation of $\operatorname{Thr}^{172}-\mathrm{AMPK} \alpha$, at least in rat skeletal muscle (in vivo) and in vitro using L6 myotubes ${ }^{30,31}$. The putative mechanism of acetate in the regulation of skeletal muscle fat oxidation, is through rapid (acute) catabolic conversion of acetate to acetyl CoA (mediated by acetyl Co A synthase), increasing the AMP/ATP ratio which subsequently increases AMPK phosphorylation. We replicated the exact same incubation time/dose as in above mentioned animal studies; however, we could not corroborate previous results. Importantly, our model is fully functional as observed by pronounced effects of Etomoxir.

Nevertheless, our model may not be the ideal system to investigate (acute) AMPK-dependent effects on fat oxidation (See Fig S2) and/or effects may be donorspecific ${ }^{48,49}$. Of note, AICAR administration in humans did not increase AMPK phosphorylation in skeletal muscle, but increased hepatic fatty acid oxidation and lowered hepatic glucose production ${ }^{50}$. Furthermore, AMPK-dependent fat oxidation might be limited in HSkMC and/or an AMPK-dependent effect on glucose uptake/oxidation may be preferentially activated ${ }^{51,52}$ as well as muscle fiber type-dependent activation of AMPK 53 in HSkMC. Although, acetate has shown AMPK-dependent fat oxidation in rodents, acetate-mediated effect on AMPK activation in skeletal muscle may be species-specific ${ }^{54}$.

Lastly, circulating acetate may modulate other metabolically active tissues in humans that may explain the in vivo increments in whole-body fat oxidation. First, SA may increase liver/adipose tissue fat oxidative capacity, since in vitro and rodent studies have shown increments in phosphorylated AMPK and total AMPK 31, 32. In addition, acetate turnover rate is the highest in the liver of mammals as compared to other tissues (i.e. muscle and adipose tissues) indicating an important regulatory role of the liver in acetate metabolism ${ }^{55}$. Second, other gut-derived metabolites such as butyrate may increase mitochondrial function and expression of fatty acid oxidative genes as observed in mice skeletal muscle ${ }^{56,57}$. Kinetic studies demonstrated that around $24 \%$ of colonic acetate is converted via bacterial cross-feeding into butyrate in metabolically healthy adults ${ }^{58}$. Finally, SCFA might affect the secretion of gut-derived hormones such as PYY and GLP-1, which have been associated with increased whole-body fat oxidation and energy expenditure in humans ${ }^{59,60}$. In line, data from our group and others reported increments of circulating PYY levels following colonic SCFA infusion in humans ${ }^{37,61}$. In agreement, a recent study reported that human skeletal muscle and muscle 
progenitor cells express PYY and its receptors, suggesting that our observed increase in whole-body fat oxidation might be partly explained via PYY-mediated effects on muscle fat oxidation ${ }^{62}$. Future research needs to investigate this in detail.

In conclusion, our data showed no time- or dose-dependent increase of SA on endogenous or exogenous palmitate oxidation as well as no increments in AMPK phosphorylation following acute/chronic SA treatment in our human primary muscle cell model HSkMC derived from a lean insulin sensitive male donor. However, we cannot exclude that our previously reported in vivo effect of colonic acetate administration on fat oxidation in overweight individuals might be partly explained by the effect of other gut-derived metabolites and their signaling pathways (i.e. via PYY) on muscle fat oxidation or by direct effects of SCFA (i.e. butyrate) on other tissues (i.e. liver and adipose tissue). Furthermore, fat oxidation might be donor-dependent and/or species-specific. 


\section{References}

1. Engin A. The Definition and Prevalence of Obesity and Metabolic Syndrome. Adv Exp Med Biol. 2017;960:1-17. Epub 2017/06/07. doi: 10.1007/978-3319-48382-5_1. PubMed PMID: 28585193.

2. Stinkens R, Goossens GH, Jocken JW, Blaak EE. Targeting fatty acid metabolism to improve glucose metabolism. Obes Rev. 2015;16(9):715-57. Epub 2015/07/17. doi: 10.1111/obr.12298. PubMed PMID: 26179344.

3. Canfora EE, Jocken JW, Blaak EE. Short-chain fatty acids in control of body weight and insulin sensitivity. Nat Rev Endocrinol. 2015;11(10):577-91. doi: 10.1038/nrendo.2015.128.

4. Canfora EE, Meex RCR, Venema K, Blaak EE. Gut microbial metabolites in obesity, NAFLD and T2DM. Nat Rev Endocrinol. 2019. Epub 2019/01/24. doi: 10.1038/s41574-019-0156-z. PubMed PMID: 30670819.

5. Conlon MA, Bird AR. The Impact of Diet and Lifestyle on Gut Microbiota and Human Health. Nutrients. 2015;7(1):17-44. doi: 10.3390/nu7010017. PubMed PMID: PMC4303825.

6. Marchesi JR, Adams DH, Fava F, Hermes GDA, Hirschfield GM, Hold G, et al. The gut microbiota and host health: a new clinical frontier. Gut. 2015.

7. Canfora EE, Blaak EE. Acetate: a diet-derived key metabolite in energy metabolism: good or bad in context of obesity and glucose homeostasis? Curr Opin Clin Nutr Metab Care. 2017. Epub 2017/08/11. doi: 10.1097/mco.0000000000000408. PubMed PMID: 28795972.

8. Cummings JH, Pomare EW, Branch WJ, Naylor CP, Macfarlane GT. Short chain fatty acids in human large intestine, portal, hepatic and venous blood. Gut. 1987;28(10):1221-7. doi: 10.1136/gut.28.10.1221. PubMed PMID: 3678950.

9. Müller M, Hernández MAG, Goossens GH, Reijnders D, Holst JJ, Jocken JWE, et al. Circulating but not faecal short-chain fatty acids are related to insulin sensitivity, lipolysis and GLP-1 concentrations in humans. Scientific Reports. 2019;9(1):12515. doi: 10.1038/s41598-019-48775-0.

10. Ferchaud-Roucher V, Pouteau E, Piloquet H, Zaïr Y, Krempf M. Colonic fermentation from lactulose inhibits lipolysis in overweight subjects. Am J Physiol Endocrinol Metab. 2005;289(4):E716-20. Epub 2005/09/10. doi: 10.1152/ajpendo.00430.2004. PubMed PMID: 16150956.

11. Pouteau E, Vahedi K, Messing B, Flourié B, Nguyen P, Darmaun D, et al. Production rate of acetate during colonic fermentation of lactulose: a stableisotope study in humans. Am J Clin Nutr. 1998;68(6):1276-83. Epub 1998/12/10. doi: 10.1093/ajcn/68.6.1276. PubMed PMID: 9846859.

12. Luo J, Rizkalla SW, Alamowitch C, Boussairi A, Blayo A, Barry JL, et al. Chronic consumption of short-chain fructooligosaccharides by healthy subjects decreased basal hepatic glucose production but had no effect on insulin- 
stimulated glucose metabolism. Am J Clin Nutr. 1996;63(6):939-45. Epub 1996/06/01. doi: 10.1093/ajcn/63.6.939. PubMed PMID: 8644690.

13. Boll EV, Ekstrom LM, Courtin CM, Delcour JA, Nilsson AC, Bjorck IM, et al. Effects of wheat bran extract rich in arabinoxylan oligosaccharides and resistant starch on overnight glucose tolerance and markers of gut fermentation in healthy young adults. Eur J Nutr. 2016;55(4):1661-70. Epub 2015/07/15. doi: 10.1007/s00394015-0985-z. PubMed PMID: 26169871.

14. Canfora EE, van der Beek CM, Hermes GDA, Goossens GH, Jocken JWE, Holst JJ, et al. Supplementation of Diet With Galacto-oligosaccharides Increases Bifidobacteria, but Not Insulin Sensitivity, in Obese Prediabetic Individuals. Gastroenterology. 2017;153(1):87-97.e3. Epub 2017/04/12. doi: 10.1053/j.gastro.2017.03.051. PubMed PMID: 28396144.

15. Neis EPJG, van Eijk HMH, Lenaerts K, Olde Damink SWM, Blaak EE, Dejong CHC, et al. Distal versus proximal intestinal short-chain fatty acid release in man. Gut. 2019;68(4):764. doi: 10.1136/gutjnl-2018-316161.

16. Le Poul E, Loison C, Struyf S, Springael JY, Lannoy V, Decobecq ME, et al. Functional characterization of human receptors for short chain fatty acids and their role in polymorphonuclear cell activation. J Biol Chem. 2003;278(28):25481-9. Epub 2003/04/25. doi: 10.1074/jbc.M301403200. PubMed PMID: 12711604.

17. Brown AJ, Goldsworthy SM, Barnes AA, Eilert MM, Tcheang L, Daniels D, et al. The Orphan G protein-coupled receptors GPR41 and GPR43 are activated by propionate and other short chain carboxylic acids. J Biol Chem. 2003;278(13):113129. Epub 2002/12/24. doi: 10.1074/jbc.M211609200. PubMed PMID: 12496283.

18. Tang C, Ahmed K, Gille A, Lu S, Grone H-J, Tunaru S, et al. Loss of FFA2 and FFA3 increases insulin secretion and improves glucose tolerance in type 2 diabetes. Nat Med. 2015;21(2):173-7. doi: 10.1038/nm.3779.

19. Priyadarshini M, Villa SR, Fuller M, Wicksteed B, Mackay CR, Alquier T, et al. An Acetate-Specific GPCR, FFAR2, Regulates Insulin Secretion. Molecular Endocrinology. 2015;29(7):1055-66. doi: 10.1210/me.2015-1007.

20. Ge H, Li X, Weiszmann J, Wang P, Baribault H, Chen J-L, et al. Activation of G Protein-Coupled Receptor 43 in Adipocytes Leads to Inhibition of Lipolysis and Suppression of Plasma Free Fatty Acids. Endocrinology. 2008;149(9):4519-26. doi: 10.1210/en.2008-0059.

21. Aberdein N, Schweizer M, Ball D. Sodium acetate decreases phosphorylation of hormone sensitive lipase in isoproterenol-stimulated 3T3-L1 mature adipocytes. Adipocyte. 2014;3(2):121-5. doi: 10.4161/adip.27936. PubMed PMID: PMC3979876.

22. Jocken JWE, González Hernández MA, Hoebers NTH, van der Beek CM, Essers YPG, Blaak EE, et al. Short-Chain Fatty Acids Differentially Affect Intracellular Lipolysis in a Human White Adipocyte Model. Front Endocrinol (Lausanne). 2018;8(372). doi: 10.3389/fendo.2017.00372.

23. Sahuri-Arisoylu M, Brody LP, Parkinson JR, Parkes H, Navaratnam N, Miller AD, et al. Reprogramming of hepatic fat accumulation and 'browning' of 
adipose tissue by the short-chain fatty acid acetate. International journal of obesity (2005). 2016;40(6):955-63. Epub 2016/03/16. doi: 10.1038/ijo.2016.23. PubMed PMID: 26975441.

24. Crouse JR, Gerson CD, DeCarli LM, Lieber CS. Role of acetate in the reduction of plasma free fatty acids produced by ethanol in man. J Lipid Res. 1968;9(4):509-12.

25. Laurent C, Simoneau C, Marks L, Braschi S, Champ M, Charbonnel B, et al. Effect of acetate and propionate on fasting hepatic glucose production in humans. Eur J Clin Nutr. 1995;49(7):484-91. Epub 1995/07/01. PubMed PMID: 7588498.

26. Wolever TM, Brighenti F, Royall D, Jenkins AL, Jenkins DJ. Effect of rectal infusion of short chain fatty acids in human subjects. Am J Gastroenterol. 1989;84(9):1027-33. Epub 1989/09/01. PubMed PMID: 2773895.

27. Suokas A, Kupari M, Heikkila J, Lindros K, Ylikahri R. Acute cardiovascular and metabolic effects of acetate in men. Alcoholism, clinical and experimental research. 1988;12(1):52-8. Epub 1988/02/01. PubMed PMID: 3279860. 28. Keenan MJ, Zhou J, McCutcheon KL, Raggio AM, Bateman HG, Todd E, et al. Effects of resistant starch, a non-digestible fermentable fiber, on reducing body fat. Obesity (Silver Spring). 2006;14(9):1523-34. Epub 2006/10/13. doi: 10.1038/oby.2006.176. PubMed PMID: 17030963.

29. Cani PD, Dewever C, Delzenne NM. Inulin-type fructans modulate gastrointestinal peptides involved in appetite regulation (glucagon-like peptide-1 and ghrelin) in rats. British Journal of Nutrition. 2004;92(3):521-6. doi: 10.1079/BJN20041225.

30. Maruta H, Yoshimura Y, Araki A, Kimoto M, Takahashi Y, Yamashita H. Activation of AMP-Activated Protein Kinase and Stimulation of Energy Metabolism by Acetic Acid in L6 Myotube Cells. PLoS One. 2016;11(6):e0158055e. doi: 10.1371/journal.pone.0158055. PubMed PMID: 27348124.

31. Yamashita H, Maruta H, Jozuka M, Kimura R, Iwabuchi H, Yamato M, et al. Effects of acetate on lipid metabolism in muscles and adipose tissues of type 2 diabetic Otsuka Long-Evans Tokushima Fatty (OLETF) rats. Biosci Biotechnol Biochem. 2009;73(3):570-6. Epub 2009/03/10. doi: 10.1271/bbb.80634. PubMed PMID: 19270372.

32. Sakakibara S, Yamauchi T, Oshima Y, Tsukamoto Y, Kadowaki T. Acetic acid activates hepatic AMPK and reduces hyperglycemia in diabetic KK-A(y) mice. Biochem Biophys Res Commun. 2006;344(2):597-604. Epub 2006/04/25. PubMed PMID: 16630552.

33. Kondo T, Kishi M, Fushimi T, Kaga T. Acetic Acid Upregulates the Expression of Genes for Fatty Acid Oxidation Enzymes in Liver To Suppress Body Fat Accumulation. Journal of Agricultural and Food Chemistry. 2009;57(13):59826. doi: 10.1021/jf900470c.

34. Smith PM, Howitt MR, Panikov N, Michaud M, Gallini CA, Bohlooly YM, et al. The microbial metabolites, short-chain fatty acids, regulate colonic Treg cell homeostasis. Science. 2013;341(6145):569-73. Epub 2013/07/06. doi: 
10.1126/science.1241165. PubMed PMID: 23828891; PubMed Central PMCID: PMCPMC3807819.

35. Verboven K, Wouters K, Gaens K, Hansen D, Bijnen M, Wetzels S, et al. Abdominal subcutaneous and visceral adipocyte size, lipolysis and inflammation relate to insulin resistance in male obese humans. Scientific Reports. 2018;8(1):4677. doi: 10.1038/s41598-018-22962-x.

36. Hulver MW, Berggren JR, Carper MJ, Miyazaki M, Ntambi JM, Hoffman $\mathrm{EP}$, et al. Elevated stearoyl-CoA desaturase-1 expression in skeletal muscle contributes to abnormal fatty acid partitioning in obese humans. Cell metabolism. 2005;2(4):251-61. doi: 10.1016/j.cmet.2005.09.002.

37. van der Beek CM, Canfora EE, Lenaerts K, Troost FJ, Damink S, Holst JJ, et al. Distal, not proximal, colonic acetate infusions promote fat oxidation and improve metabolic markers in overweight/obese men. Clin Sci (Lond). 2016;130(22):2073-82. Epub 2016/07/22. doi: 10.1042/cs20160263. PubMed PMID: 27439969.

38. Canfora EE, van der Beek CM, Jocken JWE, Goossens GH, Holst JJ, Olde Damink SWM, et al. Colonic infusions of short-chain fatty acid mixtures promote energy metabolism in overweight/obese men: a randomized crossover trial. Sci Rep. 2017;7(1):2360. Epub 2017/05/26. doi: 10.1038/s41598-017-02546-x. PubMed PMID: 28539646; PubMed Central PMCID: PMCPMC5443817.

39. Karlsson N, Fellenius E, Kiessling KH. The metabolism of acetate in the perfused hind-quarter of the rat. Acta physiologica Scandinavica. 1975;93(3):391400. Epub 1975/03/01. doi: 10.1111/j.1748-1716.1975.tb05828.x. PubMed PMID: 1146580.

40. Skutches CL, Holroyde CP, Myers RN, Paul P, Reichard GA. Plasma acetate turnover and oxidation. J Clin Invest. 1979;64(3):708-13. Epub 1979/09/01. doi: 10.1172/jci109513. PubMed PMID: 468985; PubMed Central PMCID: PMCPMC372171.

41. Mittendorfer B, Sidossis LS, Walser E, Chinkes DL, Wolfe RR. Regional acetate kinetics and oxidation in human volunteers. American Journal of Physiology-Endocrinology and Metabolism. 1998;274(6):E978-E83. doi: 10.1152/ajpendo.1998.274.6.E978. PubMed PMID: 29590564.

42. Pouteau E, Piloquet H, Maugeais P, Champ M, Dumon H, Nguyen P, et al. Kinetic aspects of acetate metabolism in healthy humans using [1-13C] acetate. American Journal of Physiology-Endocrinology and Metabolism. 1996;271(1):E58E64. doi: 10.1152/ajpendo.1996.271.1.E58. PubMed PMID: 8760082.

43. Wolfe RR, Jahoor F. Recovery of labeled CO2 during the infusion of C-1- vs C-2-labeled acetate: implications for tracer studies of substrate oxidation. The American Journal of Clinical Nutrition. 1990;51(2):248-52. doi: 10.1093/ajcn/51.2.248.

44. Neavyn MJ, Boyer EW, Bird SB, Babu KM. Sodium acetate as a replacement for sodium bicarbonate in medical toxicology: a review. J Med Toxicol. 
2013;9(3):250-4. Epub 2013/04/30. doi: 10.1007/s13181-013-0304-0. PubMed PMID: 23636658.

45. Akanji AO, Bruce MA, Frayn KN. Effect of acetate infusion on energy expenditure and substrate oxidation rates in non-diabetic and diabetic subjects. Eur J Clin Nutr. 1989;43(2):107-15. Epub 1989/02/01. PubMed PMID: 2651106.

46. Ruppin H, Bar-Meir S, Soergel KH, Wood CM, Schmitt MG, Jr. Absorption of short-chain fatty acids by the colon. Gastroenterology. 1980;78(6):1500-7. Epub 1980/06/01. PubMed PMID: 6768637.

47. Robertson MD, Bickerton AS, Dennis AL, Vidal H, Frayn KN. Insulinsensitizing effects of dietary resistant starch and effects on skeletal muscle and adipose tissue metabolism. Am J Clin Nutr. 2005;82(3):559-67. Epub 2005/09/13. doi: 10.1093/ajcn.82.3.559. PubMed PMID: 16155268.

48. Bajpeyi S, Myrland CK, Covington JD, Obanda D, Cefalu WT, Smith SR, et al. Lipid in skeletal muscle myotubes is associated to the donors' insulin sensitivity and physical activity phenotypes. Obesity (Silver Spring, Md). 2014;22(2):426-34. Epub 2013/09/10. doi: 10.1002/oby.20556. PubMed PMID: 23818429.

49. Ukropcova B, McNeil M, Sereda O, de Jonge L, Xie H, Bray GA, et al. Dynamic changes in fat oxidation in human primary myocytes mirror metabolic characteristics of the donor. J Clin Invest. 2005;115(7):1934-41. doi: 10.1172/JCI24332. PubMed PMID: 16007256.

50. Boon H, Bosselaar M, Praet SF, Blaak EE, Saris WH, Wagenmakers AJ, et al. Intravenous AICAR administration reduces hepatic glucose output and inhibits whole body lipolysis in type 2 diabetic patients. Diabetologia. 2008;51(10):1893900. Epub 2008/08/19. doi: 10.1007/s00125-008-1108-7. PubMed PMID: 18709353.

51. Sakoda H, Ogihara T, Anai M, Fujishiro M, Ono H, Onishi $\mathrm{Y}$, et al. Activation of AMPK is essential for AICAR-induced glucose uptake by skeletal muscle but not adipocytes. Am J Physiol Endocrinol Metab. 2002;282(6):E1239-44. Epub 2002/05/15. doi: 10.1152/ajpendo.00455.2001. PubMed PMID: 12006353.

52. Musi N, Goodyear LJ. AMP-activated protein kinase and muscle glucose uptake. Acta physiologica Scandinavica. 2003;178(4):337-45. Epub 2003/07/17. doi: 10.1046/j.1365-201X.2003.01168.x. PubMed PMID: 12864738.

53. Tobias IS, Lazauskas KK, Siu J, Costa PB, Coburn JW, Galpin AJ. Sex and fiber type independently influence AMPK, TBC1D1, and TBC1D4 at rest and during recovery from high-intensity exercise in humans. Journal of applied physiology (Bethesda, Md : 1985). 2020;128(2):350-61. Epub 2020/01/03. doi: 10.1152/japplphysiol.00704.2019. PubMed PMID: 31895596.

54. Kjøbsted R, Hingst JR, Fentz J, Foretz M, Sanz M-N, Pehmøller C, et al. AMPK in skeletal muscle function and metabolism. FASEB journal : official publication of the Federation of American Societies for Experimental Biology. 2018;32(4):1741-77. Epub 2018/01/05. doi: 10.1096/fj.201700442R. PubMed PMID: 29242278.

55. Knowles SE, Jarrett IG, Filsell OH, Ballard FJ. Production and utilization of acetate in mammals. Biochem J. 1974;142(2):401-11. Epub 1974/08/01. doi: 
10.1042/bj1420401. PubMed PMID: 4441381; PubMed Central PMCID: PMCPMC1168292.

56. Hong J, Jia Y, Pan S, Jia L, Li H, Han Z, et al. Butyrate alleviates high fat diet-induced obesity through activation of adiponectin-mediated pathway and stimulation of mitochondrial function in the skeletal muscle of mice. Oncotarget. 2016;7(35):56071-82. doi: 10.18632/oncotarget.11267. PubMed PMID: 27528227.

57. Frampton J, Murphy K, Frost G, Chambers E. Short-chain fatty acids as potential regulators of skeletal muscle metabolism and function. Nature Metabolism. 2020. doi: 10.1038/s42255-020-0188-7.

58. Boets E, Gomand SV, Deroover L, Preston T, Vermeulen K, De Preter V, et al. Systemic availability and metabolism of colonic-derived short-chain fatty acids in healthy subjects: a stable isotope study. J Physiol. 2017;595(2):541-55. doi: 10.1113/JP272613. PubMed PMID: PMC5233652.

59. Pannacciulli N, Bunt JC, Koska J, Bogardus C, Krakoff J. Higher fasting plasma concentrations of glucagon-like peptide 1 are associated with higher resting energy expenditure and fat oxidation rates in humans. Am J Clin Nutr. 2006;84(3):556-60. Epub 2006/09/09. doi: 10.1093/ajcn/84.3.556. PubMed PMID: 16960169.

60. Karra E, Chandarana K, Batterham RL. The role of peptide YY in appetite regulation and obesity. J Physiol. 2009;587(1):19-25. Epub 2008/12/08. doi: 10.1113/jphysiol.2008.164269. PubMed PMID: 19064614.

61. Freeland KR, Wolever TMS. Acute effects of intravenous and rectal acetate on glucagon-like peptide-1, peptide $Y Y$, ghrelin, adiponectin and tumour necrosis factor-alpha. The British journal of nutrition. 2010;103(3):460-6. doi: 10.1017/s0007114509991863. PubMed PMID: 19818198.

62. Gheller BJ, Blum JE, Merritt EK, Cummings BP, Thalacker-Mercer AE. Peptide YY (PYY) Is Expressed in Human Skeletal Muscle Tissue and Expanding Human Muscle Progenitor Cells. Frontiers in physiology. 2019;10:188-. doi: 10.3389/fphys.2019.00188. PubMed PMID: 30890955. 


\section{Supplementary Material}

4 Hour cytotoxicity

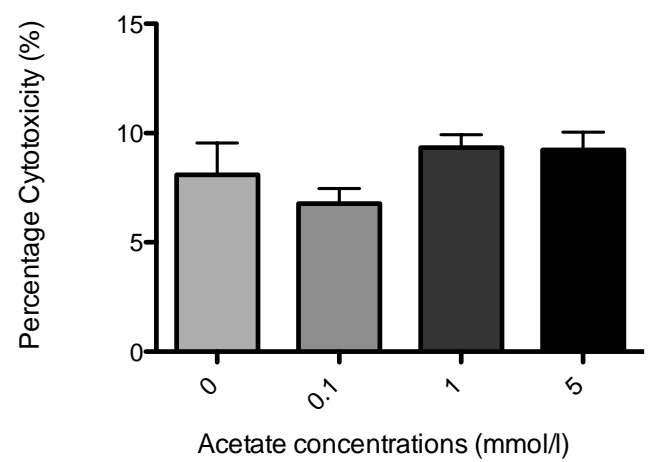

24 Hour cytotoxicity

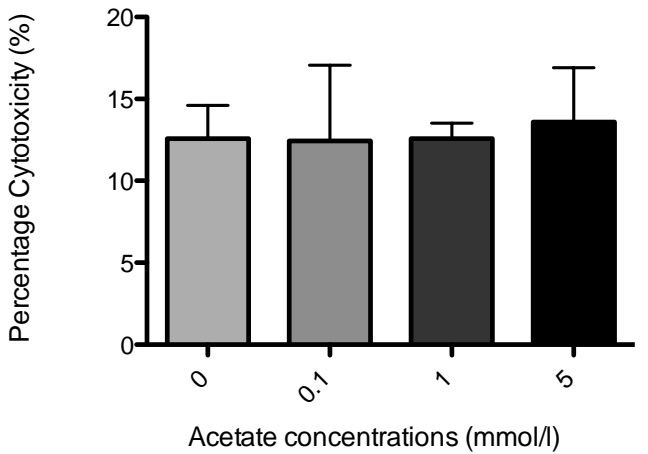

Supplementary figure 1. Cytotoxicity effect of SA on HSkMC. Cytotoxicity percentage without $(0 \mathrm{mmol} / \mathrm{l})$ and with (0.1, 1 and $5 \mathrm{mmol} / \mathrm{l}) \mathrm{SA}$ following 4- and 24-hour incubations. Data expressed as mean and standard deviation. Group differences were tested with Kruskal-Wallis. Percentage of cytotoxicity after SA was calculated in comparison to Triton-X 100, which is a positive control (100\% lysis).

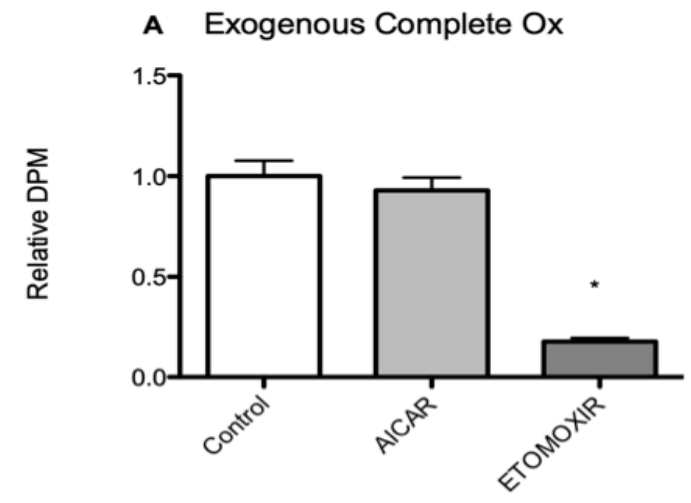

C Endogenous Complete Ox

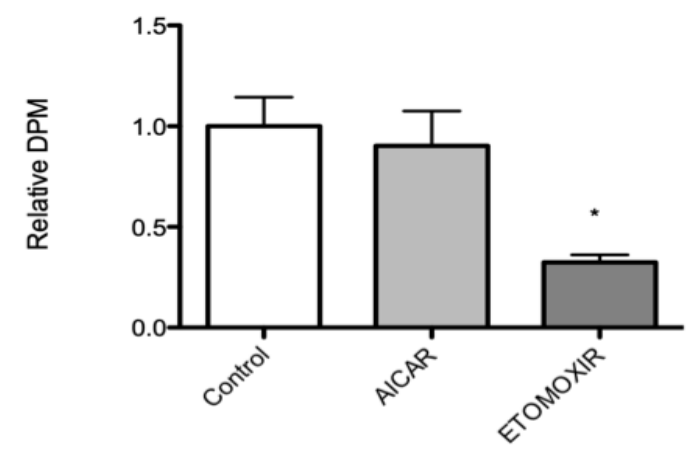

B Exogenous Incomplete Ox

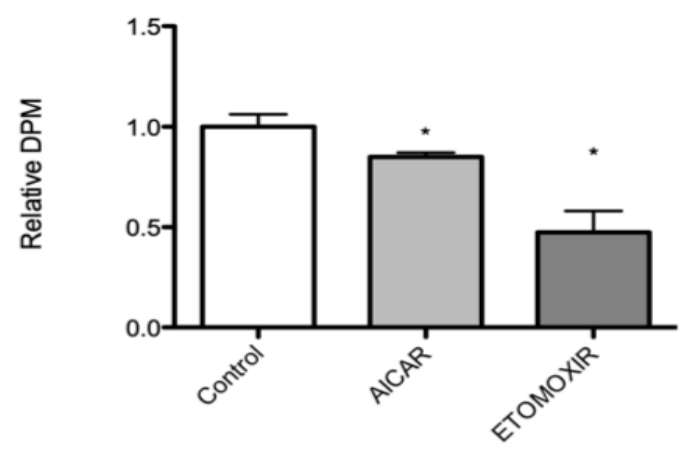

D Endogenous Incomplete Ox

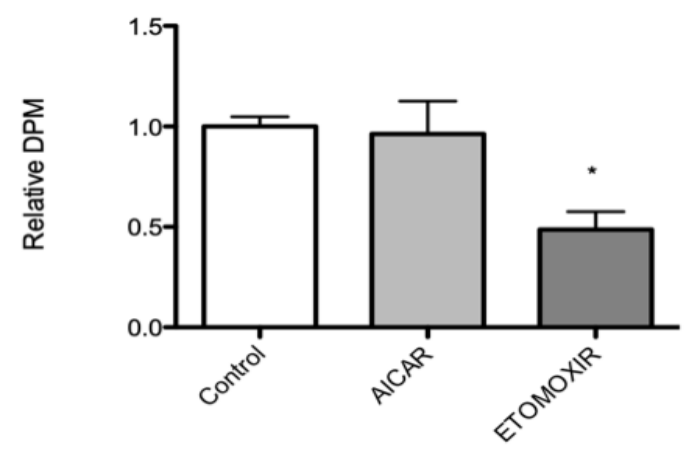

Supplementary figure 2. Muscle cell model exogenous and endogenous fat oxidation fat oxidation in HSkMC. (A) Complete $\left({ }^{14} \mathrm{CO}_{2}\right)$ and (B) incomplete (ASM) ${ }^{14} \mathrm{C}$-palmitate exogenous oxidation was measured following 4-h incubation with AICAR $(1 \mathrm{mmol} / \mathrm{l})$ and etomoxir $(100 \mu \mathrm{mol} / \mathrm{l})(1$ experiment $)$. Next, after $24 \mathrm{~h}$ pre-incubation with ${ }^{14} \mathrm{C}$-palmitate, $(\mathrm{C})$ complete $\left({ }^{14} \mathrm{CO}_{2}\right)$ and (D) incomplete (ASM) ${ }^{14} \mathrm{C}$-palmitate endogenous fat oxidation was measured following 4-h incubation with AICAR $(1 \mathrm{mmol} / \mathrm{l})$ and etomoxir $(100 \mu \mathrm{mol} / \mathrm{l})$. Data expressed as relative to control treated cells. Statistical significance using Mann-Whitney test compared to control $(0 \mathrm{mmol} / \mathrm{l})$ indicated as asterisk $\left({ }^{*}\right)$ when $\mathrm{P}<0.05$ 

Time (hrs)
0.5
4
6

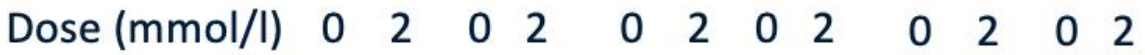

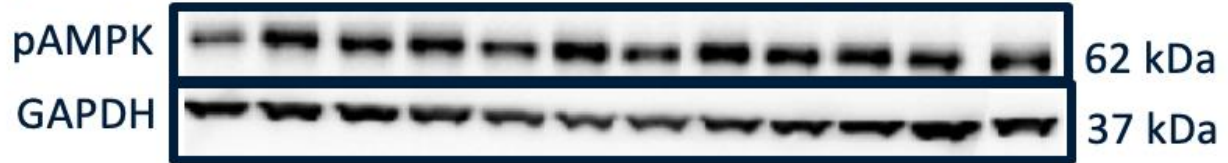

Supplementary figure 3. Time dependent effect of AICAR on AMPK phosphorylation in HSkMC. Control and AICAR ( $2 \mathrm{mmol} / \mathrm{l})$ treated cells following 0.5, 4- and 6-hours incubations. After incubation, cells were lysed with RIPA buffer and samples were subjected to western analysis for phosphorylated AMPK (Thr ${ }^{172}$ $A M P K \alpha, 62 k D$ band) and GAPDH (37kD band) was used as a loading control.
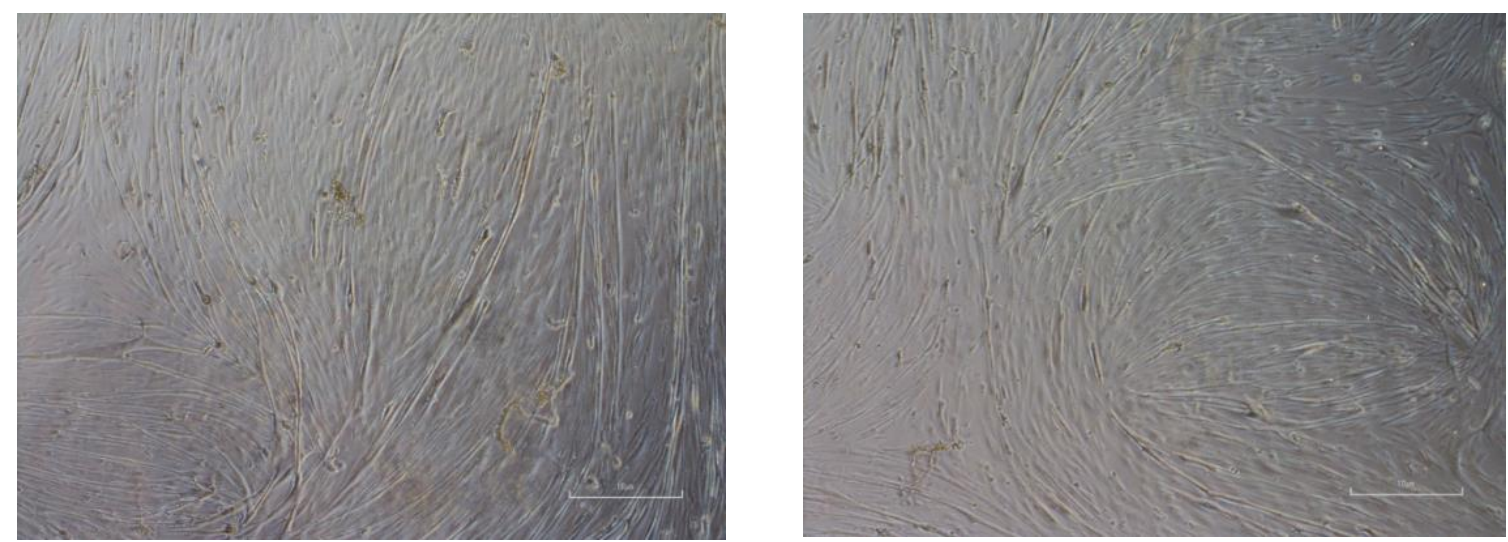

Supplementary figure 4. Fully differentiated myotubes at day 8-11 of differentiation. Pictures taken immediately prior to fat oxidation experiments or cell lysis for western blot. 


\section{CHAPTER 7}

\section{Short-chain fatty acids differentially affect intracellular lipolysis in a human white adipocyte model}

Running Title: SCFA and intracellular lipolysis

Johan W.E. Jocken, Manuel A. González Hernández, Nicole T.H. Hoebers, Christina M. van der Beek, Yvonne P.G. Essers, Ellen E. Blaak, Emanuel E. Canfora

Front. Endocrinol., 11 January 2018; 8:372 


\begin{abstract}
Background \& Aims: Gut-derived short-chain fatty acids (SCFA), formed by microbial fermentation of dietary fibres, are believed to be involved in the aetiology of obesity and diabetes. Previous data from our group showed that colonic infusions of physiologically relevant SCFA mixtures attenuated wholebody lipolysis in overweight men. To further study potential mechanisms involved in the antilipolytic properties of SCFA, we aimed to investigate the in vitro effects of SCFA incubations on intracellular lipolysis and signalling using a human white adipocyte model, the hMADS cells.
\end{abstract}

Methods: hMADS adipocytes were incubated with mixtures of acetate, propionate and butyrate or single SCFA (acetate, propionate and butyrate) in concentrations of $1 \mu \mathrm{mol} / \mathrm{L}$ and $1 \mathrm{mmol} / \mathrm{L}$. Glycerol release and lipase activation was investigated during basal conditions and following $\beta$-adrenergic stimulation.

Results: SCFA mixtures high in acetate and propionate decreased basal glycerol release, when compared to control $(P<0.05)$, whilst mixtures high in butyrate had no effect. Also, $\beta$-adrenergic receptor mediated glycerol release was not significantly altered following incubation with SCFA mixtures. Incubation with only acetate decreased basal $(1 \mu \mathrm{mol} / \mathrm{L})$ and $\beta$-adrenergically $(1 \mu \mathrm{mol} / \mathrm{L}$ and 1 $\mathrm{mmol} / \mathrm{L})$ mediated glycerol release as compared to control $(P<0.05)$. In contrast, butyrate $(1 \mu \mathrm{mol} / \mathrm{L})$ slightly increased basal and $\beta$-adrenergically mediated glycerol release compared with control $(P<0.05)$, whilst propionate had no effect on lipolysis. The antilipolytic effect of acetate was accompanied by a reduced phosphorylation of hormone sensitive lipase (HSL) at serine residue 650. In addition, inhibition of Gi G proteins following pertussis toxin treatment prevented the antilipolytic effect of acetate.

Conclusion: The present data demonstrated that acetate was mainly responsible for the antilipolytic effects of SCFA and acts via attenuation of HSL phosphorylation in a Gi coupled manner in hMADS adipocytes. Therefore, the modulation of colonic and circulating acetate may be an important target to modulate human adipose tissue lipid metabolism. 


\section{Introduction}

Increasing evidence suggests that the human gut microbiota and its products are key players in host metabolism, body weight, and insulin sensitivity, thereby contributing to the aetiology of obesity and related disorders ${ }^{1}$. The gut microbiota can ferment indigestible nutrients into short-chain fatty acids (SCFA), of which acetate, propionate and butyrate are the most abundant ${ }^{2}$. Of note, these SCFA can be taken up by the epithelial lining of the gut and released into the blood stream ${ }^{3}$. They may thereby act as important signalling molecules between gut microbiota and host physiology, by exerting effects on energy and substrate metabolism such as on adipogenesis and lipolysis in the adipose tissue ${ }^{4}$.

Disturbances in adipose tissue function, characterised by a reduced capacity to store lipids, seem to play a major role in the development of insulin resistance and type 2 diabetes mellitus in humans ${ }^{5}$. Under normal healthy conditions, the adipose tissue is an important buffering organ for daily postprandial fatty acid (FA) fluxes when endogenous lipolysis is inhibited. The adipose tissue thereby prevents excessive supply of lipids to nonadipose tissues such as liver, skeletal muscle and pancreas. This buffering action may be impaired under obese insulin resistant conditions ${ }^{6,7}$, resulting in increased circulating lipids and ectopic fat storage in nonadipose tissues, thereby provoking disturbances in insulin signalling and substrate metabolism ${ }^{8,9}$.

SCFA may affect adipose tissue lipid buffering capacity by affecting intracellular lipolysis, the process of hydrolysis of stored triacylglycerol into one molecule of glycerol and three FA molecules, and may thereby affect circulating lipid concentrations ${ }^{4}$. Indeed, already decades ago, a decrease in plasma free fatty acids (FFA) after a single oral acetate ingestion was observed, pointing to an antilipolytic role of acetate ${ }^{10}$. The more recent identification of the two pertussis toxin (PTX)-sensitive inhibitory G (Gi) protein-coupled receptors (GPR) for SCFA, Free Fatty Acid Receptor 3 (FFAR3, also known as GPR41) and FFAR2 (also known as GPR43) in human adipose tissue ${ }^{11}$, has led to renewed interest in the lipolytic properties of SCFA. In addition, a direct association between the SCFA/FFAR signalling pathway and lipolytic activity in murine adipocytes was recently discovered ${ }^{12}$. Treatment of differentiated murine 3T3-L1 adipocytes with acetate and propionate in a range between $0.1-0.3 \mathrm{mmol} / \mathrm{L}$ exhibited FFAR2 activation and a reduction in intracellular lipolytic activity as assessed by a decreased release of glycerol in the culture medium ${ }^{12}$. In contrast, incubation of 3T3-L1 adipocytes with supraphysiological concentrations of propionate $(20 \mathrm{mmol} / \mathrm{L})$ or butyrate $(5$ $\mathrm{mmol} / \mathrm{L}$ ) resulted in enhanced glycerol release ${ }^{13}$. However, in murine 3T3-L1 adipocytes only FFAR2, and not FFAR3, is expressed ${ }^{14-17}$. Therefore, it remains to be determined, whether these findings extend to human adipocytes, in which both FFAR3 and FFAR2 are expressed ${ }^{11,18}$. Thus, further investigation on the role of SCFA in human adipocyte lipolysis is urgently warranted. 
We recently observed that colonic infusions of mixtures of acetate, propionate and butyrate, in ratios and concentrations that can be reached after dietary fibre intake, attenuated whole-body lipolysis in overweight normoglycaemic men ${ }^{19}$. Therefore, the aim of the present study was to elucidate whether an altered intracellular adipocyte lipolytic rate is responsible for the antilipolytic effect of SCFA found in vivo, as well as to further investigate underlying mechanisms. Hence, we investigated the in vitro effects of incubation with SCFA mixtures and single SCFA on intracellular lipolysis in a human white adipocyte model, the human multipotent adipose tissue-derived stem (hMADS) cells. To study whether these effects are mediated via Gi-coupled receptors, we investigated the effect of SCFA on lipase activation and performed PTX-mediated inhibition of FFARs. 


\section{Material and Methods}

\section{Cell culture}

hMADS cells, a validated human white adipocyte model to study lipid metabolism ${ }^{20}$, were obtained from human subcutaneous adipose tissue biopsies and differentiated into the adipogenic lineage. As described previously by Jocken et al. ${ }^{21}$, cells were seeded at a density of 2000 cells per $\mathrm{cm}^{2}$ and kept in proliferation medium (Dulbecco's Modified Eagle's Medium (DMEM) and Ham's F-12 Nutrient Mixture (Gibco, Blijswijk, NL, USA), 10\% fetal bovine serum (Bodinco BV, Alkmaar, NL, USA) and 50 units per $\mathrm{ml}$ penicillin (Gibco), $50 \mu \mathrm{g} / \mathrm{ml}$ of streptomycin (Gibco)). At 70-80\% confluence $250 \mu \mathrm{mol} / \mathrm{L}$ IBMX (Sigma, St Louis, MI, USA) and $5 \mu \mathrm{mol} / \mathrm{L}$ rosiglitazone (Enzo Life Sciences, Raamsdonksveer, NL, USA) were added to induce adipogenic differentiation. The lipolytic experiments were carried out between days 12-14 of the differentiation.

Pooled hMADS cells were derived from male human donors with a large range in BMI $\left(20-40 \mathrm{~kg} / \mathrm{m}^{2}\right)$ and glucometabolic status. The male donors were aged between 35-70 years and participated in two different clinical trials performed (ClinicalTrials.gov, NCT02241421 and NCT02598544). The study protocols were approved by the Medical Ethical Committee Jessa hospital, Hasselt and Hasselt University, Belgium, and by the Medical Ethical Committee of Maastricht University Medical Center, Maastricht, The Netherlands. All procedures were according to the declaration of Helsinki (revised version, October 2008).

\section{Lipolysis experiment}

Free glycerol release analysis

To study effects of SCFA mixtures on the basal glycerol release, hMADS adipocytes were incubated for 6 hours with $300 \mu \mathrm{L}$ DMEM $3 \%$ fatty-acid free BSA (Sigma-Aldrich, St. Louis, United States) supplemented with or without a mixture high in acetate containing $80 \%$ acetate, $10 \%$ propionate and $10 \%$ butyrate (80:10:10), a SCFA mixture containing $60 \%$ acetate, $20 \%$ propionate and $20 \%$ butyrate (60:20:20), a mixture high in propionate containing $40 \%$ acetate, $35 \%$ propionate and $25 \%$ butyrate (40:35:25) and a mixture high in butyrate containing $40 \%$ acetate, $25 \%$ propionate and $35 \%$ butyrate (40:25:35) in final concentrations of $1 \mathrm{mmol} / \mathrm{L}$ or $1 \mu \mathrm{mol} / \mathrm{L}$ for 6 hours.

To study effects of single SCFA on the basal (non-stimulated) glycerol release, hMADS adipocytes were incubated with $300 \mu \mathrm{L}$ DMEM $3 \%$ fatty-acid free BSA (Sigma-Aldrich) supplemented with or without acetate (Merck, Darmstadt, Germany), propionate (Sigma Aldrich) or butyrate (Sigma Aldrich) at a final concentration of $1 \mathrm{mmol} / \mathrm{L}$ and $1 \mu \mathrm{mol} / \mathrm{L}$ concentrations for 6 hours.

To examine effects of single SCFA or SCFA mixtures on the $\beta$-adrenergic receptor mediated glycerol release, 30 minutes after the initiation of SCFA incubation the non-selective $\beta$-agonist isoprenaline (ISO) was added at a final 
concentration of $1 \mu \mathrm{mol} / \mathrm{L}$.

After 6 hours incubation, the plates were placed on ice to stop the reactions, and subsequently $250 \mu \mathrm{L}$ supernatant was removed and directly snap-frozen in liquid nitrogen and stored at $-80{ }^{\circ} \mathrm{C}$ until analysis. The glycerol concentrations were quantified using a commercial fluorometric assay (EnzyChrome ${ }^{\mathrm{TM}}$ Adipolysis assay kit, BioAssay Systems, Hayward, United States).

Gene expression of FFAR3 and FFAR2 in hMADS adipocytes

To determine the FFAR3 and FFAR2 mRNA expression, total RNA was extracted from hMADS adipocytes at day 0, 2, 7, 10 and 12 using TRIzol reagent (Invitrogen) and SYBR-Green based real-time PCRs were performed using an iCylcer (Biolegio, Nijmegen, The Netherlands; primer sequences see Table 1). Results were normalized for $18 \mathrm{~S}$ ribosomal RNA (calculating delta-delta Ct values). Ct values ranged from 27 - 33 for FFAR3/2 and 6-9 for $18 S$ ribosomal RNA.

Table 1: Primer sequences

\begin{tabular}{lll}
\hline Gene & Forward $\left(\mathbf{5}^{\prime} \rightarrow \mathbf{3}^{\prime}\right)$ & Reverse $\left(\mathbf{3}^{\prime} \boldsymbol{\rightarrow} \mathbf{5}^{\prime}\right)$ \\
\hline 18S & AGTTAGCATGCCAGAGTCTCG & TGCATGGCCGTTCTTAGTTG \\
FFAR3 (GPR41) & TTCACCACCATCTATCTCACCG & GGAACTCCAGGTAGCAGGTC \\
FFAR2 (GPR43) & CCGTGCAGTACAAGCTCTCC & CTGCTCAGTCGTGTTCAAGTATT \\
\hline
\end{tabular}

Abbreviations: FFAR, free fatty acid receptor; GPR, G-protein coupled receptor.

\section{Western blotting}

To study the effects of acetate on the protein expression and activation (phosphorylation) of the key lipolytic enzymes adipose triglyceride lipase (ATGL) and hormone-sensitive lipase (HSL), hMADS adipocytes were incubated with 300 $\mu \mathrm{L}$ DMEM 3 \% BSA supplemented with or without acetate at a final concentration of $1 \mu \mathrm{mol} / \mathrm{L}$ for one hour. In addition, $30 \mathrm{~min}$ after the start of the acetate incubation, ISO was added to the medium at a final concentration of $1 \mu \mathrm{mol} / \mathrm{L}$ to investigate the effects of acetate on the $\beta$-adrenergic receptor mediated HSL phosphorylation. Following one-hour incubation, cells were washed twice with ice-cold phosphate-buffered saline (PBS), and cells were homogenized in radioimmunoprecipitation assay (RIPA) buffer supplemented with a protease and phosphatase inhibitor cocktail (Cell Signaling, Leiden, The Netherlands). $20 \mu \mathrm{g}$ solubilized proteins were separated on a Criterion TGX precast gel (Bio-Rad), transferred using the Trans Blot Turbo transfer system (Bio-Rad), and incubated with primary antibodies. The HSL antibody was a kind gift from Prof. C. Holm (Lund University, Lund, Sweden). The ATGL (No.: 2138) and the pHSL at serine residue 650 (rat SER660) (No.: 4126) antibodies were both obtained from Cell Signaling Technology, Leiden, The Netherlands. To determine the FFAR3 (antibody No.: 103718, Abcam, Cambridge, UK) and FFAR2 (antibody No.: 131003, Abcam, Cambridge, UK) protein expression, total protein was extracted from 
hMADS adipocytes at day $0,2,4,7,9,11$ and 14 . Secondary antibodies (antibody No.: P0161, antibody No.: P0399).

Effect of inhibition of Gi proteins using pertussis toxin

To study the putative involvement of the Gi-type G-protein coupled FFARs in the acetate-induced inhibition of the lipolytic response, we incubated hMADS adipocytes for 6 hours with acetate in a concentration of $1 \mu \mathrm{mol} / \mathrm{L}$ during basal and ISO stimulated conditions (see protocol above), with or without supplementation of PTX for the whole 6 hours to the medium at a final concentration of $100 \mathrm{ng} / \mathrm{mL}$ (No.: P7208, Sigma Aldrich). The PTX experiments were conducted according the Guidelines of the European Chemicals Agency (Helsinki, Finland).

\section{Statistical analysis}

Values are expressed as mean \pm SD. Significance was determined using the nonparametric Mann Whitney U-test when comparing two groups (single SCFA, western blotting and PTX experiment) or the Kruskal-Wallis H-test when comparing more groups (SCFA mixtures). In case of significant Kruskal-Wallis Htest, Dunns posthoc test was performed. Statistics were performed using the GraphPad Prism 5.0a software package (GraphPad Software, San Diego, CA, USA) and $P<0.05$ (two-sided $P$-value) was considered statistically significant. 


\section{Results}

\section{SCFA mixtures high in acetate and propionate decrease basal adipocyte glycerol release in vitro}

We investigated whether the observed decrease in systemic glycerol found in our in vivo study ${ }^{19}$ is related to an attenuated adipocyte intracellular lipolysis. Therefore, we incubated hMADS adipocytes for 6 hours with $1 \mu \mathrm{mol} / \mathrm{L}$ and 1 $\mathrm{mmol} / \mathrm{L}$ of SCFA mixtures. The SCFA mixtures high in acetate and propionate (80:10:10, 60:20:20 and 40:35:25) decreased the basal (non-stimulated) glycerol release, as compared to control $(P<0.05$, Figure $1 \mathrm{~A})$. The SCFA mixture high in butyrate (40:25:35) did not significantly affect basal glycerol release (Figure 1A). In contrast to the decreased basal lipolysis, $\beta$-adrenergic receptor mediated glycerol release was not significantly affected following incubation with all SCFA mixtures in concentrations of $1 \mu \mathrm{mol} / \mathrm{L}$ and $1 \mathrm{mmol} / \mathrm{L}$ (Figure 1B).

\section{Single SCFA differentially affect adipocyte glycerol release in vitro}

Subsequently, we studied whether one particular SCFA was responsible for the observed antilipolytic effect. Therefore, we incubated hMADS adipocytes for 6 hours with acetate, propionate and butyrate in concentrations of $1 \mu \mathrm{mol} / \mathrm{L}$ and 1 $\mathrm{mmol} / \mathrm{L}$. Incubation with $1 \mu \mathrm{mol} / \mathrm{L}$ acetate decreased basal glycerol release when compared to control cells $(P<0.05$, Figure $2 \mathrm{~A})$. In addition, acetate blunted the $\beta$ adrenergic receptor mediated glycerol release in concentrations of $1 \mathrm{mmol} / \mathrm{L}$ and $1 \mu \mathrm{mol} / \mathrm{L}$, when compared to control $(P<0.05$, Figure $2 \mathrm{~B})$. In contrast, $1 \mu \mathrm{mol} / \mathrm{L}$ butyrate treatment slightly increased basal $(P<0.05$, Figure $2 \mathrm{~A})$ and $\beta$-adrenergic receptor mediated glycerol release $(P<0.01$, Figure $2 B)$, when compared to control treated cells. Neither in the basal state nor during $\beta$-adrenergic receptor stimulation, a significant difference between propionate and control treated adipocytes were observed (Figure $2 \mathrm{~A}$ and B). 
A

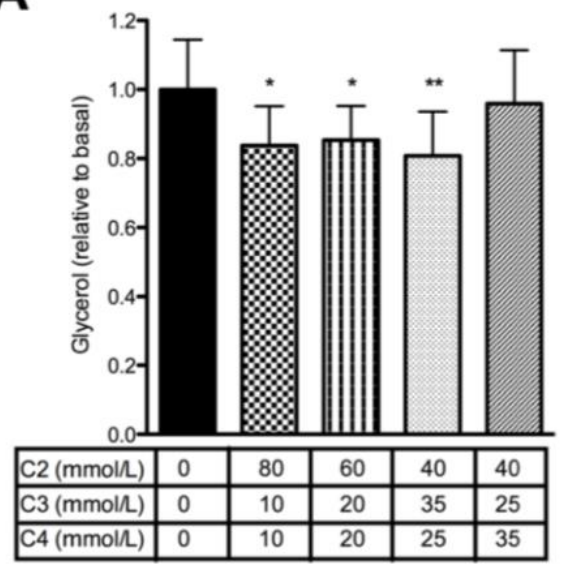

B

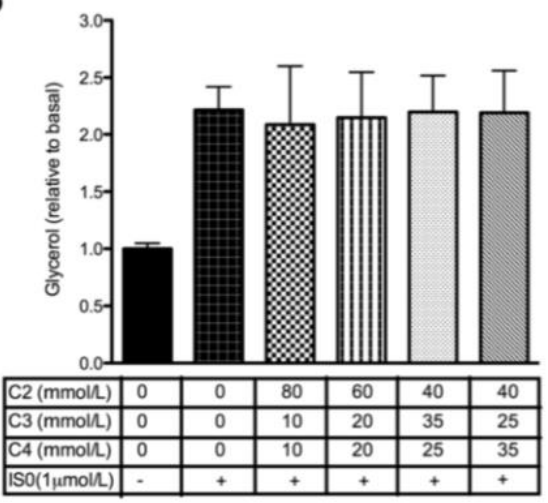

Incubation

time: $6 \mathrm{~h}$
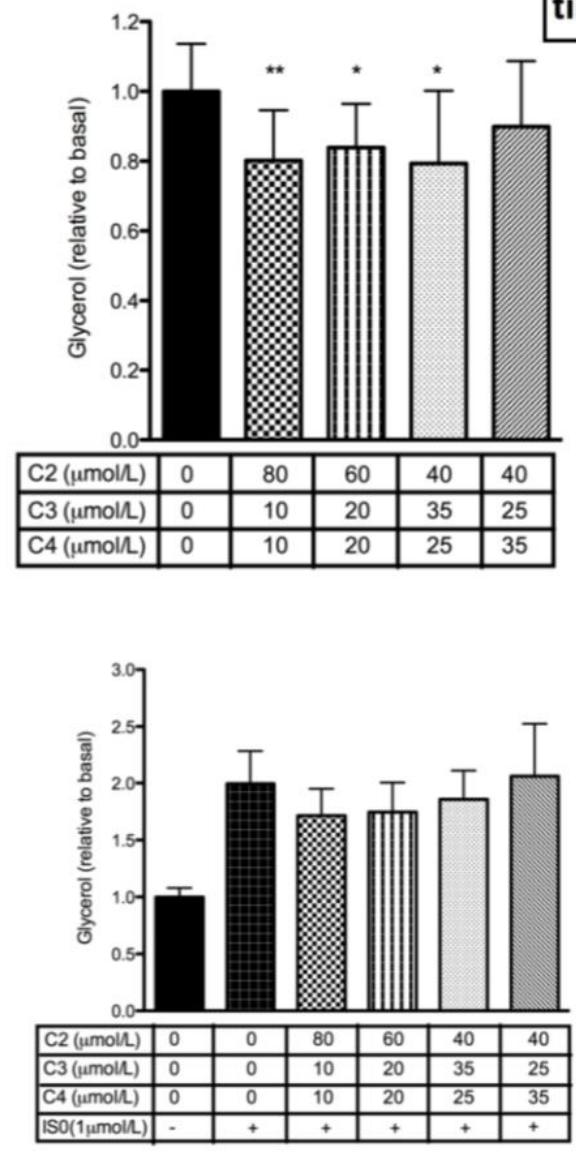

Figure 1. Effect of SCFA mixtures on basal and $\beta$-adrenergic receptor stimulated glycerol release in hMADS adipocytes

A: Basal (non-stimulated) glycerol concentrations during $6 \mathrm{~h}$ incubation with 1 $\mathrm{mmol} / \mathrm{L}$ or $1 \mu \mathrm{mol} / \mathrm{L} \mathrm{SCFA}$ mixtures including acetate (C2), propionate (C3) and butyrate (C4); B: Effect of $6 \mathrm{~h}$ incubation with $1 \mathrm{mmol} / \mathrm{L}$ or $1 \mu \mathrm{mol} / \mathrm{L}$ SCFA mixtures including acetate (C2), propionate (C3) and butyrate (C4) on $\beta$-adrenergic receptor stimulated $(1 \mu \mathrm{mol} / \mathrm{L}$ ISO) glycerol release; Values are given as means \pm SD $(n=4$ 6 independent experiments). Statistical significance compared to basal indicated as asterisk $\left(^{*}\right)$ when $P<0.05$ and double asterisk $\left({ }^{*}\right)$ when $P<0.01$. 
A
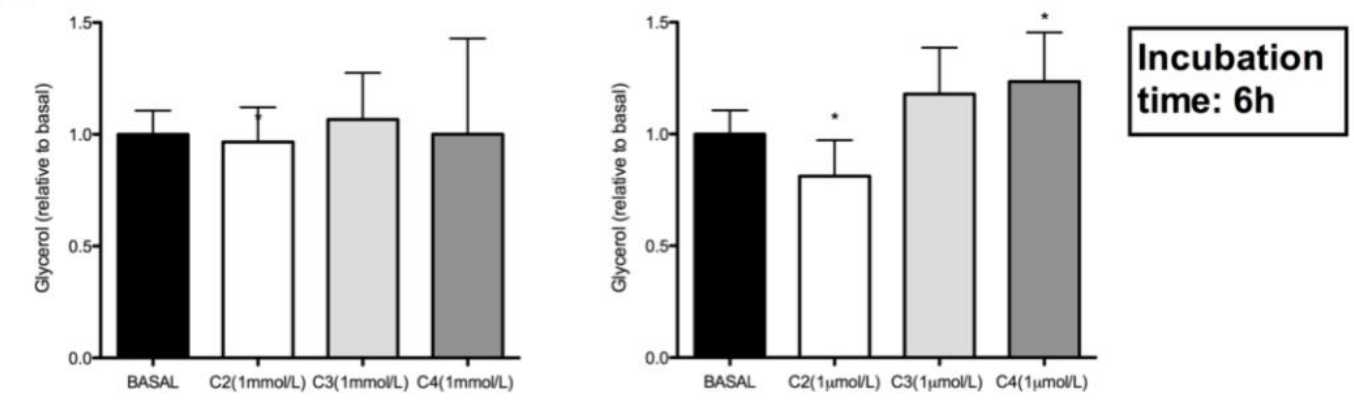

B
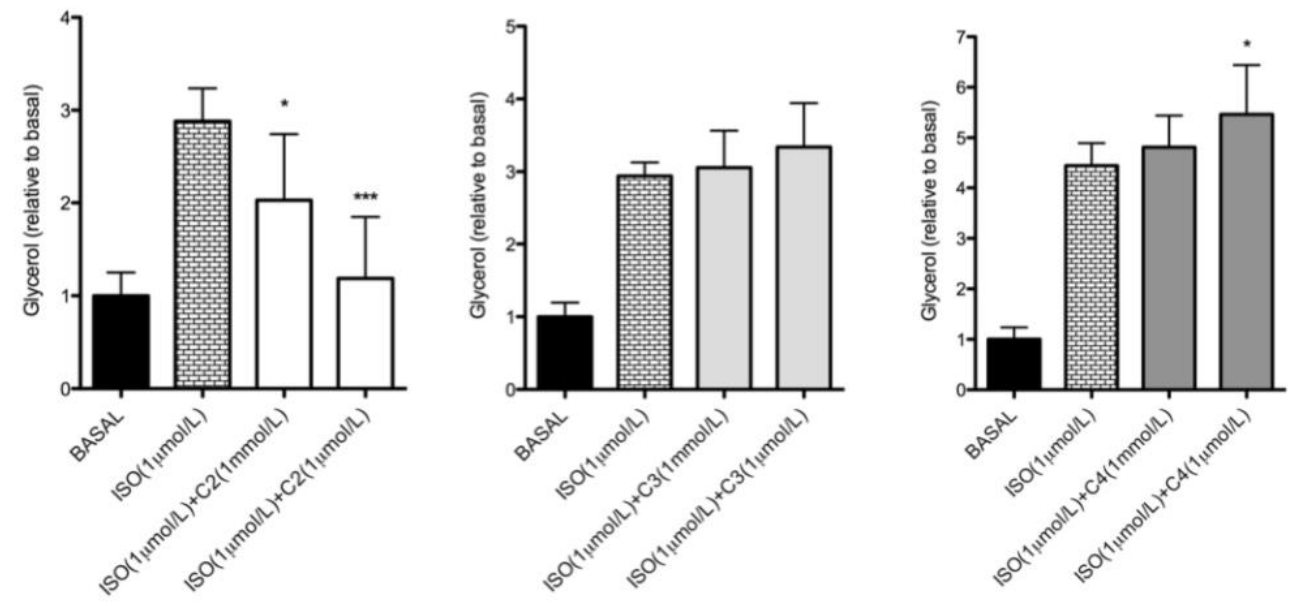

Figure 2. Effect of single SCFA on basal and $\beta$-adrenergic receptor stimulated glycerol release in hMADS adipocytes

A: Basal (non-stimulated) glycerol concentrations during $6 \mathrm{~h}$ incubation with 1 $\mathrm{mmol} / \mathrm{L}$ or $1 \mu \mathrm{mol} / \mathrm{L}$ acetate (C2), propionate (C3) or butyrate (C4); B: Effect of $6 \mathrm{~h}$ incubation with $1 \mathrm{mmol} / \mathrm{L}$ or $1 \mu \mathrm{mol} / \mathrm{L}$ acetate (C2), propionate (C3) or butyrate (C4) on $\beta$-adrenergic receptor stimulated ( $1 \mu \mathrm{mol} / \mathrm{L}$ ISO) glycerol release; Values are given as means $\pm S D(n=4-7$ independent experiments). Statistical significance as compared to basal indicated as asterisk $\left(^{*}\right)$ when $P<0.05$ and as triple asterisk $\left.{ }^{* * *}\right)$ when $P<0.001$.

\section{Acetate attenuates HSL phosphorylation in adipocytes}

Since the above data indicated that mainly acetate is the driver of the antilipolytic effect of SCFA in human adipocytes, we subsequently investigated the underlying mechanisms in more detail by quantification of key enzymes involved in intracellular lipolysis, including ATGL, HSL and phosphorylated HSL (pHSL). No differences of acetate on total HSL or ATGL protein content were observed (Figure 3A). As indicated in Figure 3B, treatment of hMADS adipocytes with $1 \mu \mathrm{mol} / \mathrm{L}$ acetate resulted in a reduction in the relative amount of phosphorylation of HSL on the serine 650 as compared to control non-treated cells 
$(P<0.01$, Figure 3B). As expected, the phosphorylation of HSL on the serine 650 increased by 5-6 fold in the presence of ISO, as compared to non-stimulated adipocytes (Figure $3 \mathrm{~A}$ and $\mathrm{B}$ ). However, pre-treatment of hMADS adipocytes with $1 \mu \mathrm{mol} / \mathrm{L}$ acetate and ISO resulted in reduction in the relative amount of pHSL(SER650) Compared to ISO stimulation alone ( $P<0.05$, Figure 3B).

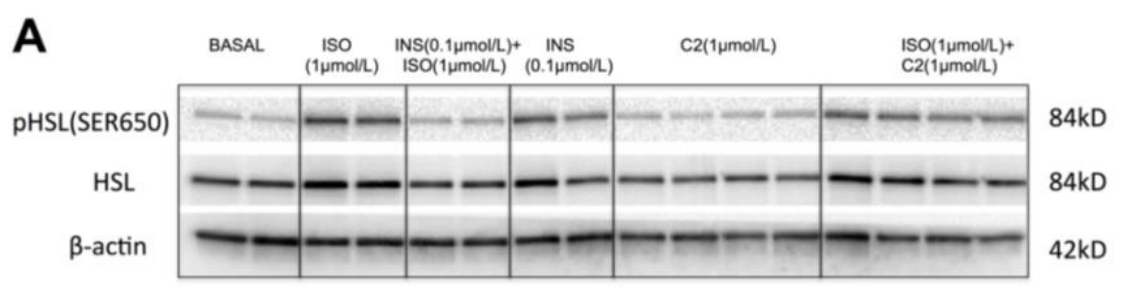

B

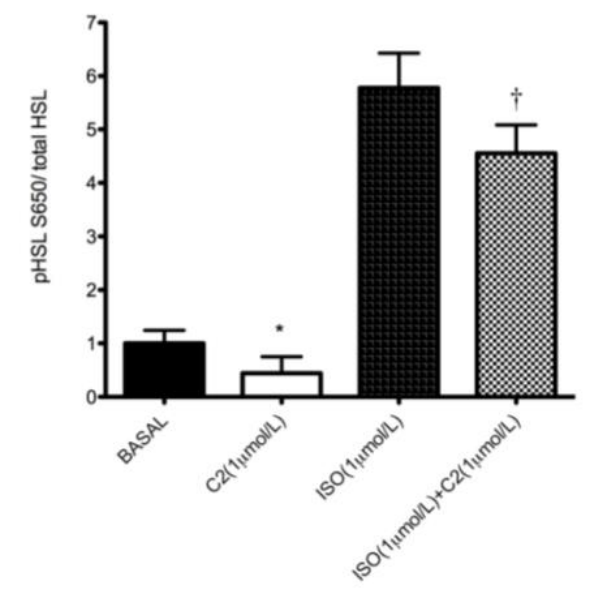

Incubation time: $1 \mathrm{~h}$

Figure 3. Acetate attenuates HSL (SER 650) phosphorylation in hMADS adipocytes

A: Representative western blot showing that $1 \mu \mathrm{mol} / \mathrm{L}$ acetate $(\mathrm{C} 2)$ reduced the relative amount of HSL phosphorylated on serine 650 in the presence of isoprenaline (ISO). In this blot, insulin (INS) was used as a control. See for corresponding entire blots supplementary figure $1 \mathbf{B}$ : Quantification of western blot using ImageLab 3.0 normalized to total HSL $(n=4)$. Values are given as means \pm SD. Statistical significance as compared to basal indicated as asterisk $\left(^{*}\right)$ when $P<0.05$; and as compared to ISO as dagger ( $\dagger$ ) when $P<0.05$.

\section{PTX treatment prevents the antilipolytic effect of acetate in hMADS cells}

Finally, we investigated the involvement of inhibitory Gi G protein coupled receptors in this acetate-mediated antilipolytic effect. Both FFAR3 and FFAR2, the major SCFA receptors, were expressed at the RNA (Figure 4A) and protein (Figure 4B) level in our hMADS cells, and expression increased during adipogenic differentiation with a maximal expression at day 12 and 14 (see Figure 4; Figure S3C in Supplementary Material for FFAR2 protein expression in hMADS at day 14). 
Next, hMADS adipocytes were incubated with or without PTX, which irreversibly blocks Gi function, thereby inhibiting both FFAR3 and FFAR2 in our hMADS adipocytes. Of interest, PTX prevented the acetate-mediated $(1 \mu \mathrm{mol} / \mathrm{L})$ decrease in basal and $\beta$-adrenergic receptor stimulated glycerol release $(P<0.01$, Figure 5).

A
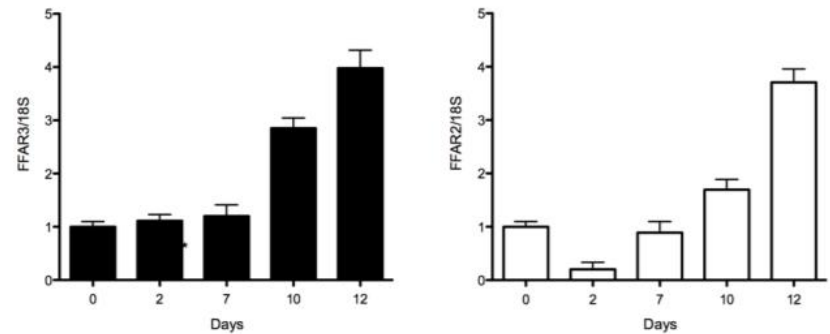

B
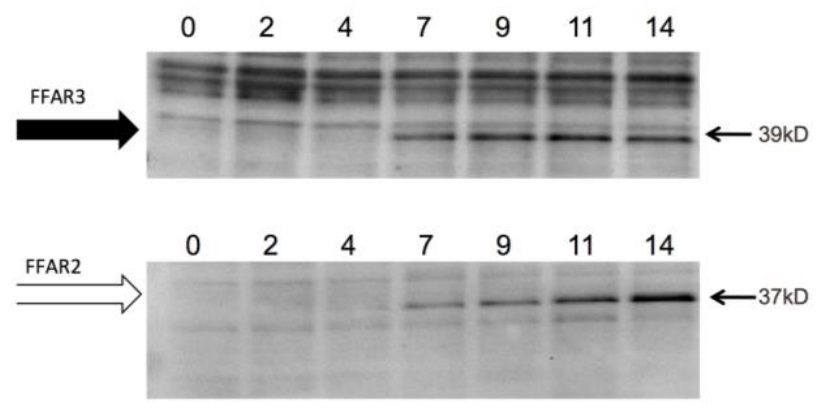

Figure 4: FFAR3 and FFAR2 are expressed at the RNA and protein level in hMADS adipocytes

A: FFAR3/2 mRNA expression during adipocyte differentiation (day 0 - 12). B: FFAR3/2 protein expression during adipocyte differentiation (day 0 - 14) $(n=1)$, See for corresponding entire blots supplementary figure 2 . 


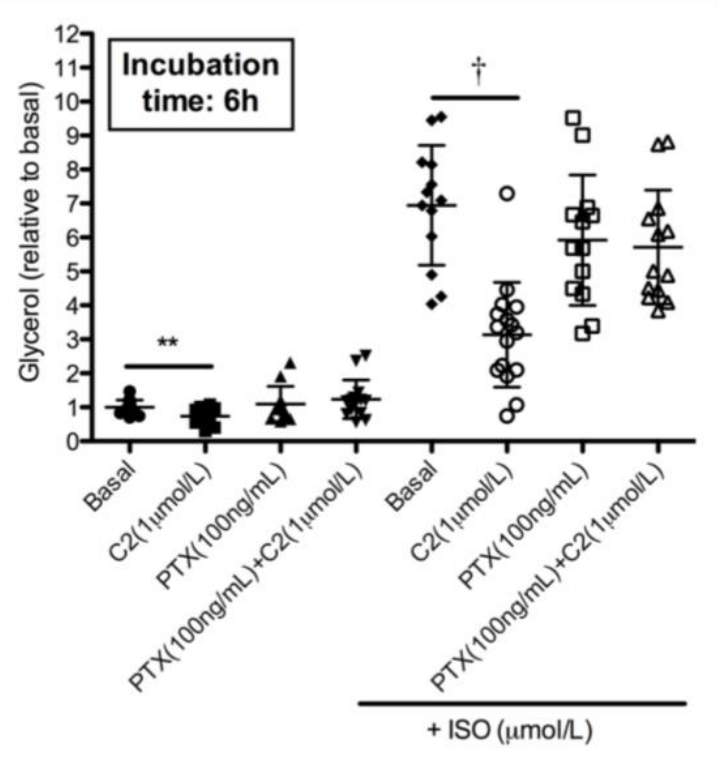

Figure 5: Pertussis toxin (PTX) abrogated acetate-induced inhibition (1 $\mu \mathrm{mol} / \mathrm{L})$ of ISO-mediated glycerol release in hMADS adipocytes

Values are given as individual points and means \pm SD $(n=4$ independent experiments). Statistical significance when compared to basal indicated as asterisk $\left.{ }^{* *}\right)$ when $P<0.01$; and as compared to isoprenaline (ISO) as dagger ( $\dagger$ ) when $P<0.001$. 


\section{Discussion}

This study provides new insight in the effects of SCFA on human adipocyte lipolysis. We previously showed that acute colonic administration of three physiological-relevant SCFA mixtures, and subsequent elevated circulating acetate concentrations, reduced circulating glycerol concentration in overweight males, indicative of a reduced whole-body lipolysis ${ }^{19}$. However, to further investigate whether the reduction in whole-body lipolysis was related to a putative SCFA effect on white adipocyte intracellular lipolysis, we performed several in vitro experiments using our validated hMADS adipocyte model. Our present in vitro study in hMADS adipocytes demonstrated that mainly acetate had antilipolytic effects, which was accompanied by a reduced phosphorylation of HSL (at SER650). Incubation with the Gi inhibitor PTX prevented the acetatemediated antilipolytic effect, suggesting that this antilipolytic effect may be mediated through an acetate-FFAR coupled signalling pathway (for an schematic overview see figure 6).

This study demonstrated that SCFA mixtures at physiological $(1 \mu \mathrm{mol} / \mathrm{L})$ and more supraphysiological $(1 \mathrm{mmol} / \mathrm{L})$ concentrations attenuate intracellular lipolysis in human adipocytes. Furthermore, by subsequently incubating human adipocytes with single SCFA, we demonstrated that the intestinally and systemically most abundant SCFA acetate seems to be the main driver of this antilipolytic effect. Acetate is the most abundant circulating SCFA and is found in serum and plasma mean concentrations varying from $5 \mu \mathrm{mol} / \mathrm{L}$ up to $220 \mu \mathrm{mol} / \mathrm{L}$, depending on the nutritional status ${ }^{3,22-24}$. Propionate and butyrate are found at much lower maximal mean concentrations of $13 \mu \mathrm{mol} / \mathrm{L}$ and $12 \mu \mathrm{mol} / \mathrm{L}$, respectively 3, 22, 23. However, no human data are available reflecting SCFA concentrations that reach the adipose tissue via their capillaries. Based on the scarcely available data on circulating SCFA concentrations, an acetate to propionate to butyrate ratio of 80:10:10 and 60:20:20 could resemble physiologically circulating concentrations and the SCFA concentrations used in this study might be in a physiological $(1 \mu \mathrm{mol} / \mathrm{L})$ to supraphysiological $(1 \mathrm{mmol} / \mathrm{L})$ range. Interestingly, the most pronounced effects on lipolysis were found with an acetate concentration of $1 \mu \mathrm{mol} / \mathrm{L}$, which thus seems to be lower than circulating concentrations. Therefore, it would be of major interest to measure actual acetate concentrations in adipose tissue capillaries or interstitial fluids. To the knowledge of the authors, no data on this are available, which would be very interesting to detect via for example microdialysis techniques. 


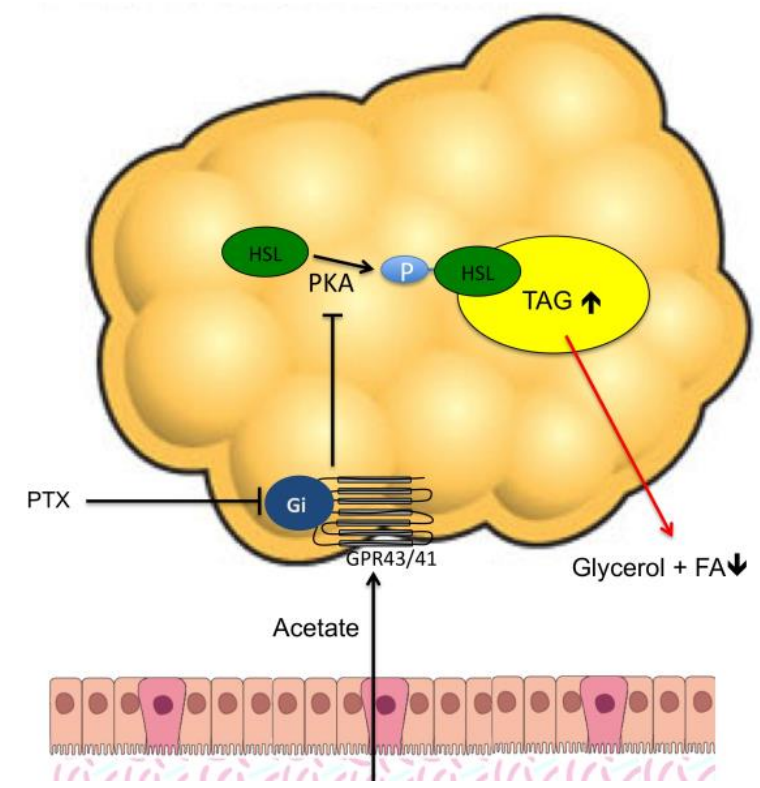

Figure 6: Proposed mechanism of the acetate-mediated antilipolytic effect in human adipocytes.

The blunted FA and glycerol release during acetate incubation is accompanied by a reduced phosphorylation of HSL(SER650), indicating a role for protein kinase A (PKA) in this antilipolytic process. The FFAR inhibitor PTX prevents the acetate-mediated antilipolytic effect, indicating a role for a Gi protein coupled receptor mechanism (i.e. FFAR3 and/or FFAR2) in human adipocyte lipolysis. In addition, we observed that the antilipolytic effect of acetate was accompanied by a reduced phosphorylation of HSL at the serine 650, a major protein kinase A (PKA) regulatory site. In accordance with this observation, Aberdein et al. ${ }^{25}$ indicated that treatment of murine 3T3-L1 adipocytes with supraphysiological concentrations $(4 \mathrm{mmol} / \mathrm{L})$ of sodium acetate reduced the $\beta$ adrenergic receptor stimulated non-esterified FA release and decreased HSL phosphorylation at another PKA regulatory site (serine 563) ${ }^{25}$. Ge et al. ${ }^{12}$ showed that treatment of 3T3-L1 adipocytes with acetate and propionate in a range between $0.1-0.3 \mathrm{mmol} / \mathrm{L}$ reduced the basal and $\beta$-adrenergic receptor stimulated intracellular lipolytic activity as assessed by a decreased release of glycerol in the culture medium ${ }^{12}$. In contrast to the antilipolytic effect of acetate, we observed a slightly increased basal lipolytic response after butyrate treatment in hMADS adipocytes. Comparable results were reported by Rumberger et al. ${ }^{13}$ showing an increased basal lipolytic response (glycerol release) following incubation of murine 3T3-L1 adipocytes with $5 \mathrm{mmol} / \mathrm{L}$ butyrate ${ }^{13}$. However, the underlying mechanism of this lipolytic effect of butyrate needs further investigation in hMADS cells. Together, these results suggest that acetate is the main driver for the antilipolytic effects of SCFA, which is accompanied by an attenuated HSL phosphorylation in both, murine and human adipocytes. 
Finally, we observed that the antilipolytic effect of acetate might be FFAR dependent. We first showed in accordance to other reports in human adipocyte models ${ }^{18}$ that both, FFAR3 and FFAR2 transcripts and protein are expressed in our hMADS adipocyte model, and that both increased during adipogenic differentiation. Furthermore, we showed that the effects of acetate are abrogated with co-incubation of PTX. PTX is a well-known FFAR inhibitor and irreversible inactivates Gi proteins. Thereby, these data suggest that the acetate effects were mediated via a Gi protein receptor-PKA pathway. In line, a previous study in murine 3T3-L1 adipocytes has shown that the lipolytic effect of acetate was mediated by activation of FFAR2 ${ }^{12}$. However, further investigations are warranted to elucidate whether SCFA effects on human intracellular adipocyte lipolysis are mediated mainly via FFAR2 and/or FFAR3, using specific human knockdown models. In particular the role of FFAR3/2 protein should be further investigated via the use of knockdown and overexpression in human adipocyte models.

Furthermore, evidence is increasing that metabolic phenotype should be considered in future lipolysis studies. Present literature provides evidence that obesity-related metabolic disturbances, such as insulin resistance, are linked to differences in circulating acetate levels and acetate-induced metabolic responses. For example, in our acute studies ${ }^{19,24}$ we included overweight and obese individuals with average fasting acetate concentrations of approximately $20-50$ $\mu \mathrm{mol} / \mathrm{L}$, whereas in another study of our group with insulin resistant obese individuals markedly higher acetate concentrations of approximately $70-90$ $\mu \mathrm{mol} / \mathrm{L}$ have been found ${ }^{26}$. In addition, a kinetic study showed that the acetate clearance rate is lower and the half-life is longer in type 2 diabetic patients as compared to healthy normoglycaemic controls ${ }^{27}$. This suggests a disturbed uptake and/or metabolism of acetate, which might be relevant to elicit acetate-induced metabolic effects and cell signalling in peripheral tissues. Furthermore, there are indications that overweight insulin resistant compared to normoglycaemic individuals have lower acetate-induced antilipolytic responses on a whole-body level ${ }^{28}$. An acute study demonstrated that intravenously administered acetate resulted in a greater free fatty acid fall and rebound in healthy adults compared with hyperinsulinaemic individuals ${ }^{28}$. Therefore, comparing SCFA-mediated inhibition of the lipolytic response and intracellular signalling mechanism in adipocytes derived from normoglycaemic, insulin sensitive versus metabolically more compromised donors is of major interest. Here, we included cells from human adults with a wide range of BMI and glucometabolic status, therefore we did not distinguish between metabolic phenotypes, which is as limitation.

Nevertheless, this study has clinical implications. If the observed results can be translated into long-term in vivo metabolic effects, increased systemic acetate availability might improve human white adipose tissue lipid buffering capacity and reduce adipose tissue lipid spillover. This could ultimately result in 
attenuated ectopic fat accumulation and improved insulin action in insulin sensitive tissues such as skeletal muscle, pancreas and liver, preventing insulin resistance. The present study showed that acetate induces a partial inhibition of intracellular lipolysis during basal conditions. Interestingly, combined data derived from rodents and humans demonstrated that a comparable partial inhibition of intracellular lipolysis has beneficial effects on insulin sensitivity without affecting adipose tissue mass in the longer term ${ }^{29,}{ }^{30}$. In addition to elevated basal lipolysis, $\beta$-adrenergic receptor agonist sensitivity is blunted in obese insulin resistant individuals, ${ }^{31-33}$ which poses the question whether a further decrease in $ß$-adrenergically mediated lipolysis by SCFA is positive with respect to metabolic health. Thus, further research including isoprenaline concentration response curves are needed to ascertain SCFA-induced changes in efficiency or potency of $\beta$-adrenergic receptor agonists in different metabolic phenotypes.

With the present in vitro study using our human adipocyte model we explored a mechanism that might explain the previously in vivo observed antilipolytic effect of physiologically relevant SCFA mixtures ${ }^{19}$. However, other mechanisms, which might also contribute to the SCFA-induced antilipolytic effect on whole-body level, could not be excluded here. For example, in parallel to acetate concentrations, circulating peptide YY (PYY) concentrations were increased after the colonic infusions of SCFA mixtures in our in vivo experiment ${ }^{19}$. Indeed, PYY was previously recognized for its antilipolytic property in human adipocytes ${ }^{34}$. In addition, an intriguing study in rodents showed that SCFA can influence energy homeostasis including lipolysis via dorsal sympathetic ganglions and spinal pathways ${ }^{35}$.

In conclusion, we demonstrated that in particular the colonic and peripheral most abundant SCFA acetate plays an important role in the regulation of human adipose tissue lipolysis. We showed that the luminal and systemically most abundant SCFA acetate was mainly responsible for the antilipolytic response, via FFAR-mediated attenuation of HSL phosphorylation in human adipocytes. Indicating that the modulation of colonic and systemic acetate might be a target to prevent or improve insulin resistance in human. Therefore, future studies should focus on increasing nutritional strategies to enhance circulating acetate availability, for example via supplementation of specific acetogenic fibres, to improve human lipid metabolism. 


\section{References}

1. Delzenne NM, Cani PD, Everard A, Neyrinck AM, Bindels LB. Gut microorganisms as promising targets for the management of type 2 diabetes. Diabetologia. 2015;58(10):2206-17.

2. Canfora EE, Blaak EE. The role of polydextrose in body weight control and glucose regulation. Current Opinion in Clinical Nutrition \& Metabolic Care. 2015;18(4):395-400.

3. Bloemen JG, Venema K, van de Poll MC, Olde Damink SW, Buurman WA, Dejong $\mathrm{CH}$. Short chain fatty acids exchange across the gut and liver in humans measured at surgery. Clinical nutrition. 2009;28(6):657-61.

4. Canfora EE, Jocken JW, Blaak EE. Short-chain fatty acids in control of body weight and insulin sensitivity. Nat Rev Endocrinol. 2015;11(10):577-91. doi: 10.1038/nrendo.2015.128.

5. Goossens GH. The role of adipose tissue dysfunction in the pathogenesis of obesity-related insulin resistance. Physiology \& behavior. 2008;94(2):206-18.

6. Mensink M, Blaak EE, van Baak MA, Wagenmakers AJ, Saris WH. Plasma free fatty acid uptake and oxidation are already diminished in subjects at high risk for developing type 2 diabetes. Diabetes. 2001;50(11):2548-54.

7. Jocken JW, Langin D, Smit E, Saris WH, Valle C, Hul GB, et al. Adipose triglyceride lipase and hormone-sensitive lipase protein expression is decreased in the obese insulin-resistant state. Journal of Clinical Endocrinology \& Metabolism. 2007;92(6):2292-9.

8. Ravussin E, Smith SR. Increased fat intake, impaired fat oxidation, and failure of fat cell proliferation result in ectopic fat storage, insulin resistance, and type 2 diabetes mellitus. Annals of the New York Academy of Sciences. 2002;967(1):363-78.

9. Britton KA, Fox CS. Ectopic Fat Depots and Cardiovascular Disease. Circulation. 2011;124(24):e837-e41.

10. Crouse JR, Gerson CD, DeCarli LM, Lieber CS. Role of acetate in the reduction of plasma free fatty acids produced by ethanol in man. Journal of lipid research. 1968;9(4):509-12.

11. Xiong Y, Miyamoto N, Shibata K, Valasek MA, Motoike T, Kedzierski RM, et al. Short-chain fatty acids stimulate leptin production in adipocytes through the G protein-coupled receptor GPR41. Proceedings of the National Academy of Sciences of the United States of America. 2004;101(4):1045.

12. Ge H, Li X, Weiszmann J, Wang P, Baribault H, Chen JL, et al. Activation of $\mathrm{G}$ protein-coupled receptor 43 in adipocytes leads to inhibition of lipolysis and suppression of plasma free fatty acids. Endocrinology. 2008;149(9):4519.

13. Rumberger JM, Arch JR, Green A. Butyrate and other Short-Chain Fatty Acids Increase the Rate of Lipolysis in 3T3-L1 Adipocytes. PeerJ PrePrints, 2014 2167-9843. 
14. Kimura I, Ozawa K, Inoue D, Imamura T, Kimura K, Maeda T, et al. The gut microbiota suppresses insulin-mediated fat accumulation via the short-chain fatty acid receptor GPR43. Nature communications. 2013;4:1829.

15. Inoue D, Tsujimoto G, Kimura I. Regulation of energy homeostasis by GPR41. Frontiers in Endocrinology. 2014;5.

16. Hong YH, Nishimura Y, Hishikawa D, Tsuzuki H, Miyahara H, Gotoh C, et al. Acetate and propionate short chain fatty acids stimulate adipogenesis via GPCR43. Endocrinology. 2005;146(12):5092.

17. Zaibi MS, Stocker CJ, O'Dowd J, Davies A, Bellahcene M, Cawthorne MA, et al. Roles of GPR41 and GPR43 in leptin secretory responses of murine adipocytes to short chain fatty acids. FEBS letters. 2010;584(11):2381-6.

18. Vangaveti V, Rush C, Thomas L, Rasalam RR, Malabu UH, McCoombe SG, et al. Short-chain fatty acids increase expression and secretion of stromal cellderived factor-1 in mouse and human re-adipocytes. Hormones. 2014;13:532-42.

19. Canfora EE, Beek CM, Jocken JW, Goossens GH, Holst JJ, Damink SWO, et al. Colonic infusions of short-chain fatty acid mixtures promote energy metabolism in overweight/obese men: a randomized crossover trial. Scientific Reports. 2017;7(1):2360.

20. Bezaire V, Mairal A, Ribet C, Lefort C, Girousse A, Jocken J, et al. Contribution of adipose triglyceride lipase and hormone-sensitive lipase to lipolysis in hMADS adipocytes. Journal of Biological Chemistry. 2009;284(27):18282-91.

21. Jocken J, Goossens G, Popeijus H, Essers Y, Hoebers N, Blaak E. Contribution of lipase deficiency to mitochondrial dysfunction and insulin resistance in hMADS adipocytes. International Journal of Obesity. 2015.

22. Cummings JH, Pomare EW, Branch WJ, Naylor CP, Macfarlane GT. Short chain fatty acids in human large intestine, portal, hepatic and venous blood. Gut. 1987;28(10):1221-7. doi: 10.1136/gut.28.10.1221. PubMed PMID: 3678950.

23. Fernandes J, Vogt J, Wolever T. Kinetic model of acetate metabolism in healthy and hyperinsulinaemic humans. European journal of clinical nutrition. 2014.

24. van der Beek CM, Canfora EE, Lenaerts K, Troost FJ, Damink SWO, Holst JJ, et al. Distal, not proximal, colonic acetate infusions promote fat oxidation and improve metabolic markers in overweight/obese men. Clinical Science. 2016:130 (22) 2073-82.

25. Aberdein N, Schweizer M, Ball D. Sodium acetate decreases phosphorylation of hormone sensitive lipase in isoproterenol-stimulated 3T3-L1 mature adipocytes. Adipocyte. 2014;3(2):121-5. doi: 10.4161/adip.27936. PubMed PMID: PMC3979876.

26. Canfora EE, van der Beek CM. Supplementation of Diet With Galactooligosaccharides Increases Bifidobacteria, but Not Insulin Sensitivity, in Obese Prediabetic Individuals. Gastroenterology. 2017;1:11. 
27. Lim J, Henry CJ, Haldar S. Vinegar as a functional ingredient to improve postprandial glycemic control-human intervention findings and molecular mechanisms. Molecular Nutrition \& Food Research. 2016;60(8):1837-49. doi: 10.1002/mnfr.201600121.

28. Fernandes J, Vogt J, Wolever TM. Intravenous acetate elicits a greater free fatty acid rebound in normal than hyperinsulinaemic humans. European journal of clinical nutrition. 2012;66(9):1029-34.

29. Girousse A, Tavernier G, Valle C, Moro C, Mejhert N, Dinel A-L, et al. Partial inhibition of adipose tissue lipolysis improves glucose metabolism and insulin sensitivity without alteration of fat mass. PLoS biology. 2013;11(2):e1001485.

30. Schweiger M, Romauch M, Schreiber R, Grabner GF, Hütter S, Kotzbeck P, et al. Pharmacological inhibition of adipose triglyceride lipase corrects high-fat diet-induced insulin resistance and hepatosteatosis in mice. Nature communications. 2017;8:14859.

31. Large V, Hellström L, Reynisdottir S, Lönnqvist F, Eriksson P, Lannfelt L, et al. Human beta-2 adrenoceptor gene polymorphisms are highly frequent in obesity and associate with altered adipocyte beta-2 adrenoceptor function. Journal of Clinical Investigation. 1997;100(12):3005.

32. Hoffstedt J, Arner P, Hellers G, Lönnqvist F. Variation in adrenergic regulation of lipolysis between omental and subcutaneous adipocytes from obese and non-obese men. J Lipid Res. 1997;38(4):795-804.

33. Jocken J, Goossens G, van Hees A, Frayn K, van Baak M, Stegen J, et al. Effect of beta-adrenergic stimulation on whole-body and abdominal subcutaneous adipose tissue lipolysis in lean and obese men. Diabetologia. 2008;51(2):320-7.

34. Valet P, Berlan M, Beauville M, Crampes F, Montastruc J, Lafontan M. Neuropeptide $\mathrm{Y}$ and peptide $\mathrm{YY}$ inhibit lipolysis in human and dog fat cells through a pertussis toxin-sensitive $G$ protein. Journal of Clinical Investigation. 1990;85(1):291.

35. De Vadder F, Kovatcheva-Datchary P, Goncalves D, Vinera J, Zitoun C, Duchampt A, et al. Microbiota-generated metabolites promote metabolic benefits via gut-brain neural circuits. Cell. 2014;156(1):84-96. 
A

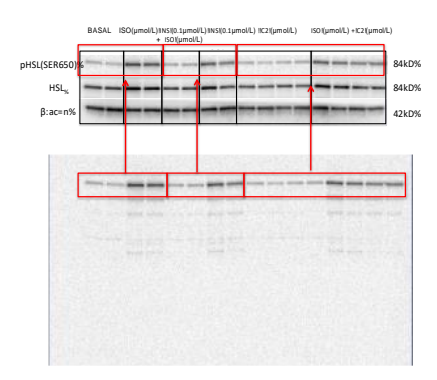

B

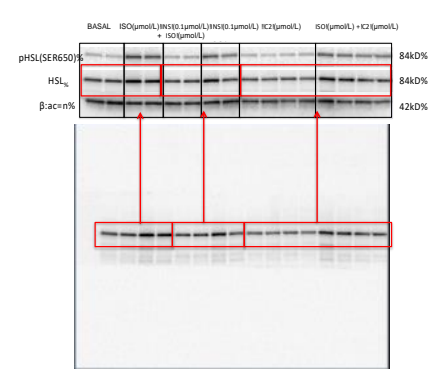

C

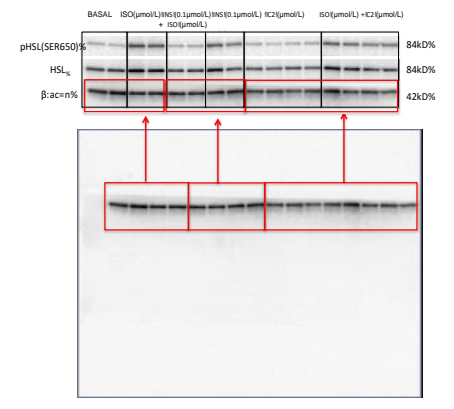

Supplementary figure 1: Corresponding blots for figure 3A Acetate attenuates HSL (SER 650) phosphorylation in hMADS adipocytes of the manuscript.
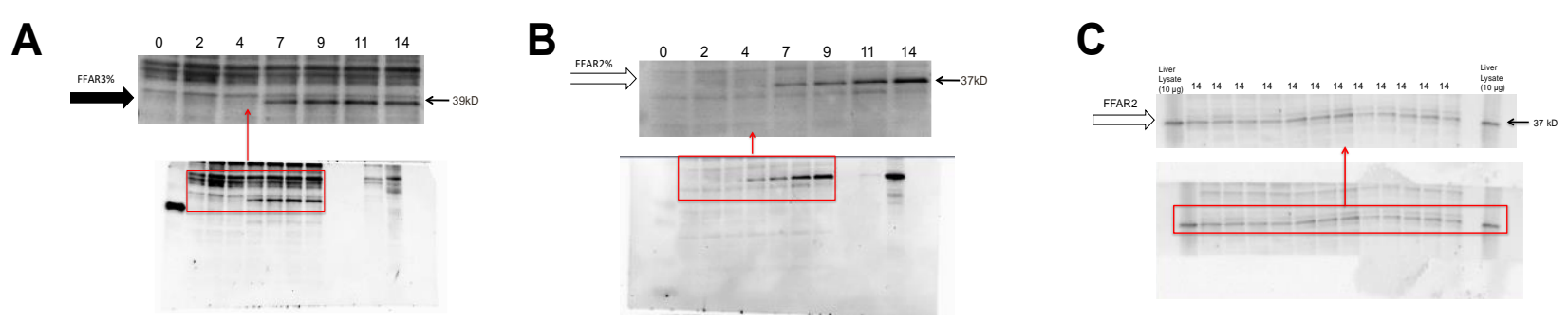

Supplementary figure 2: A and B: Corresponding blots for figure 4B FFAR3 and FFAR2 are expressed at the protein level in $h M A D S$ adipocytes of the manuscript $\mathbf{C}$ : Additional blot of FFAR2 protein expression of fully differentiated hMADS adipocytes at day 14 (as positive control $10 \mu \mathrm{g}$ human fetal liver lysate was used). 



\section{CHAPTER 8}

General Discussion 


\section{Obesity Epidemic}

Obesity and comorbidities have dramatically increased worldwide in the last decades ${ }^{1}$. Excessive body weight and the accompanying increased adiposi may result in adipose tissue dysfunction and the development of insul: resistance, Type 2 diabetes Mellitus (T2DM) and cardiovascular disease (CVD) ${ }^{\perp}$. An important factor connecting these pathophysiological conditions is the decreased adipose tissue lipid buffering capacity, which results in lipid overflow and ectopic fat deposition in non-adipose tissues (i.e. skeletal muscle, liver, pancreas, heart) ${ }^{2}$. In addition, an impaired fat oxidation unable to cope with the increased lipid supply as well as increased systemic low-grade inflammation contribute to ectopic lipid accumulation (bioactive lipid metabolites), impairments in insulin signaling (elevated proinflammatory cytokines) and the subsequent development of insulin resistance ${ }^{3-5}$.

Several surgical, pharmacological and lifestyle strategies have focused on body weight management to combat obesity and its complications. Currently, the highest prevalence (between 28-36\%) of obesity is observed in North America (i.e. Mexico), some parts of Europe and the Middle East. Although the above mentioned strategies improved throughout the last decade, the prevalence of obesity is expected to further increase in the coming decades ${ }^{6}$. Taken together, there is an urgent need to investigate additional strategies for the prevention and treatment of obesity, in particular on long-term maintenance of weight loss- or dietary intervention-induced metabolic improvements. In particular, more targeted or personalized strategies considering individual preferences, genetic, microbial and metabolic phenotypes are required to promote weight loss and improve metabolic health over longer periods of time.

Currently, scientific insights with the focus on the gut microbiota, its metabolites and its inter-organ crosstalk with peripheral tissues highlight the rationale to target the gut and its inhabitants to elicit metabolic benefits ${ }^{7}$. Based on this, interventions (i.e. pre- or probiotics) have focused on microbiota modulation to improve gut and host metabolic health ${ }^{8}$. Predominantly, research has shown that a more pronounced saccharolytic fermentation (prebiotics) in the whole colon may increase the production of postbiotic metabolites such as short chain fatty acids (SCFA). Of note, SCFA administration elicited various beneficial effects on metabolic health in several animal and in a limited number of human studies ${ }^{9-13}$. On the other hand, a less explored field is the modulation of gut microbiota through other saccharolytic metabolites (e.g lactate, succinate) as well as through proteolytic (protein) fermentation and the production of branched chain fatty acids (BCFA) with possible more adverse metabolic effects ${ }^{12}$. Although fecal BCFA are positively related to obesity in humans ${ }^{14-16}$, more studies are warranted to elucidate the underlying mechanisms. 
Furthermore, it is important to consider that gut microbiota composition and functionality ${ }^{17-25}$ and metabolic phenotype ${ }^{26-28}$ may determine the effectiveness of a dietary intervention aiming to modulate saccharolytic/proteolytic fermentation ratio.

This doctoral thesis investigated the association of fecal/plasma SCFA (acetate, propionate and butyrate), other fecal metabolites (lactate, caproate, valerate and succinate) as well as fecal BCFA (isobutyrate, isovalerate) with metabolic health parameters in well phenotyped human cohorts. In addition, we investigated, in vitro, underlying mechanisms of action of SCFA in peripheral tissue substrate metabolism using human derived primary cells from skeletal muscle and adipose tissue.

\section{Microbial metabolites derived from saccharolytic fermentation and insulin sensitivity in humans}

\section{SCFA and metabolic health}

In general, various studies have suggested that SCFA may have tissue specific effects and beneficially impact whole body insulin sensitivity and metabolic health ${ }^{29}$. For instance, muscle gastrocnemius extracts from butyrate supplemented mice (C57BL/6J) showed increments in radiolabeled palmitate oxidation by $200 \%{ }^{30}$. In line, SCFA may increase oxidative capacity in liver and muscle tissues which may work through an increased phosphorylation of AMPK as shown in in vitro and animal studies after acetate treatment ${ }^{31-33}$. In support, a human study showed that oral administration of sodium propionate increased fasting fat oxidation and resting energy expenditure independent of glucose and insulin levels in healthy individuals ${ }^{34}$. Although there is vast literature on SCFA, the majority has focused on acetate, which evidence comes mainly from animal and in vitro studies ${ }^{29}$. Therefore, in chapter 2 , we extensively reviewed the effects of acetate, the most abundant SCFA in the colon and systemic circulation, in the context of insulin sensitivity and body weight control. Briefly, an increased acetate availability in the systemic circulation resulted in an antilipolytic effects on whole body level as observed in various in vivo studies (animal and human) ${ }^{35-43}$, increments in fat oxidation and in gut hormone levels (peptide $Y Y$ and glucagon like-peptide 1 PYY/GLP-1 levels) in humans ${ }^{44}$, 45 . In addition, increased plasma acetate concentrations are positively associated with insulin sensitivity markers in humans ${ }^{46,47}$. Of note, acute colonic infusions (rich in acetate) have shown to beneficially affect whole-body human substrate and energy metabolism as reflected by increments in fasting fat oxidation and energy expenditure as well as in PYY and GLP-1 levels $44,45,48$. 
Interestingly, this effect only occurred after distal and not after proximal colonic acetate infusions ${ }^{45}$. In relation, a study in men conducted during abdominal surgery reported a higher SCFA release (highest for acetate) from distal compared to proximal colon, indicating that colonic absorption may differ between colonic segments ${ }^{49}$. Collectively, these data suggested that an increased acetate availability and absorption in the distal colon (i.e. via supplementation of slowly fermentable fibers), may be a strategy to beneficially affect whole body metabolism through the aforementioned tissue specific effects.

In this regard, a prebiotic intervention in obese/prediabetic men using an acetogenic fiber (galacto-oligosaccharides, 12-week, 15g/d) did not report changes in fecal/plasma levels of SCFA despite an observed bifidogenic effect ${ }^{50}$. This was possibly explained by increased fermentation mainly in the proximal colon and/or an insufficient acetate production, as no increments in fecal and plasma SCFA concentrations were observed after the intervention. Furthermore, research from our group recently investigated whether a mixture of fibers (long-chain inulin and resistant starch), which increased gut microbial acetate production the highest in the distal colon (using an in vitro model of the human colon) as compared to other mixtures, could increase acetate production in lean and prediabetic individuals ${ }^{51}$. This intervention indicated an increased energy expenditure, carbohydrate oxidation and postprandial insulin sensitivity in lean individuals, but not in individuals with overweight and prediabetes. These metabolic phenotype-specific effects are in line with a previous human study, in which a 4-week oral butyrate supplementation resulted in increased peripheral and hepatic insulin sensitivity in healthy lean but not in metabolic syndrome individuals ${ }^{27}$. Although these data suggest that effects may depend on metabolic phenotype with a lower or delayed response in metabolically disturbed individuals, there are a few studies that have shown that prebiotic fiber administrations improved insulin sensitivity markers in individuals with obesity and T2DM ${ }^{52,53}$.

Another approach to increase circulating acetate is through oral administrations, such as vinegar supplementations, which have shown improvements in glucose homeostasis, however, individual characteristics (metabolic phenotype and glycemic profile) may again play a role in the metabolic effects induced by acetate. Specially, vinegar supplementations (i.e. containing 6\% acetic acid) seem to be more effective in terms of improvements in glycemic control in normo- as compared to hyperinsulinemic individuals ${ }^{28}$. A further interesting aspect to consider is the microbial phenotype and its microbial community stratification in Bacteroides, Prevotella, Ruminococcus ${ }^{17}$. For instance, the Bacteroides enterotype has been linked to higher consumption of protein and saturated fats, whereas Prevotella enterotype has been associated to a lower protein intake ${ }^{54}$. Taken together, these data indicate that further studies aiming to improve metabolic health by increasing the SCFA/acetate availability in tissues such as liver, skeletal muscle and adipose tissue through fiber supplementation or vinegar 
supplementations may need to consider metabolic phenotypes and/or microbial phenotypes ${ }^{55}$.

Another potential therapeutic intervention to increase SCFA availability in the gut is through probiotic supplementations, which aim to increase SCFA producing bacteria including acetate producers 56, 57. For instance, Akkermansia muciniphila supplementation, an acetate producer and mucin degrader, may exert multiple beneficial effects by influencing host metabolism and immune system ${ }^{58}$. Of note, a recent human intervention study providing Akkermansia muciniphila showed safety, tolerability and increased insulin sensitivity markers (+28\%) in overweight-obese insulin resistant individuals ${ }^{59}$. Furthermore, a synbiotic supplementation (fructo-oligosaccharides plus 7 strains of bacteria including Bifidobacterium longum, Lactobacillus bulgaricus, Lactobacillus casei) showed improvements in insulin resistance and fasting glucose in individuals with metabolic syndrome ${ }^{60}$. Although no measurements of SCFA were performed here 58,60 , it gives an indication for future studies to investigate the role of acetate or other SCFA using gut microbiota-altering interventions to restore the SCFA producing and metabolizing capacity in metabolically compromised individuals. In summary, human interventions using pre-, pro- or synbiotics, vinegar supplementations or combinations may have the potential to increase colonic and circulating acetate to exert its whole-body and tissue specific effects (as reviewed in chapter 2). Further research should consider colonic production site and the role of the metabolic phenotype in SCFA handling. To further investigate the role of circulating acetate as well as circulating propionate and butyrate, we studied the association of fasting concentrations of SCFA with markers of insulin sensitivity in two large cohorts with well-phenotyped individuals.

\section{Do SCFA have to enter the systemic circulation to exert metabolic effects?}

As described above an increased availability of acetate in the distal colon may lead to a high acetate supply to the systemic circulation that may beneficially modulate tissue specific and whole-body metabolism. In view of the fact that previous data showed that fasting plasma acetate levels correlated with fat oxidation and energy expenditure after colonic infusions in humans ${ }^{48}$, together with positive associations of circulating acetate concentrations with insulin sensitivity markers in humans ${ }^{46,47}$, we hypothesized in chapter 3 that fasting systemic acetate concentrations positively associate with insulin sensitivity markers in the DiOGenes study. In contrast, we found a slight positive association between changes in circulating fasting acetate and changes in HOMA-IR (std $\beta$ $0.130, p=0.033$ ) after the low-calorie diet (LCD) phase in females but not in males even after adjustment for confounders (age, weight and fat free mass). Of importance, this finding should be interpreted with caution considering the 
contrasting evidence that has provided valuable insight of acetate tissue specific mechanisms ${ }^{61}$ as well as whole-body effects of acetate in humans (increments in fasting fat oxidation, energy expenditure, PYY secretion) ${ }^{44,48,62}$.

Nevertheless, these findings raise various questions about the association of circulating acetate and insulin sensitivity including the significance of the site of acetate production (gut-derived acetate, endogenously derived from liver), acetate kinetics/clearance a well as the sexual dimorphism of this relationship. First, it should be noted that circulating acetate might be the resultant of colonic acetate production as well as endogenously hepatic acetate production which may strongly depend on metabolic status. Notably, acetate and ketone bodies concentrations were determined after the LCD in the DiOGenes study during energy restricted conditions, and these concentrations partly reflect the increased hepatic ketogenesis and elevate acetate production during energy restriction ${ }^{63}$. Secondly, the sexual dimorphism in this relationship is interesting and warrants further investigation. The latter may possibly relate to sex-specific differences in tissue specific insulin resistance phenotypes, as a previous DiOGenes study reported that despite the fact that hepatic insulin resistance was lower in females compared to males, worsening of the lipid profile was associated with hepatic insulin resistance in females, but not in males ${ }^{64}$. In support to the sexual dimorphism, a previous DiOGenes analysis suggested a sex-specific modulatory role of baseline acetate in energy metabolism, since they reported a negative association with LCD-induced BMI change in morbidly obese females ( $\operatorname{std} \beta-0.23$ ) but not in males ${ }^{65}$.

Furthermore, whether sex hormones play a role in this relationship through alterations in SCFA handling and/or hepatic metabolic processes remains unresolved. In this cohort, most women were in the premenopausal state, unfortunately data on hormonal status were unavailable. Thirdly, when interpreting this data it should be considered the complexity of acetate kinetics in the colon, which depends on the microbial composition, its functionality and cross-feeding mechanisms ${ }^{55}$. More studies are warranted to investigate the relationship between acetate concentrations and insulin sensitivity further as well as acetate kinetics where microbially produced acetate and endogenously produced acetate may reflect different metabolic conditions in distinct phenotypes.

Furthermore, we recognize that fecal SCFA are often considered as a biomarker of SCFA production, however, this may have disadvantages as we investigated and discussed in chapter 4 . Here, we investigated the associations between both fecal and plasma SCFA (acetate, propionate and butyrate) with metabolic health parameters in individuals with a wide range of BMI and glucometabolic status. Our data indicated that in particular circulating fasting SCFA were associated with metabolic health parameters like circulating lipids, GLP-1 and insulin sensitivity whilst there were no associations between fecal concentrations of SCFA and metabolic health parameters ${ }^{66}$. Although various 
studies have found increased levels of fecal SCFA in individuals with obesity ${ }^{25,67,}$ 68 , these findings should be interpreted with caution in presence of the vast amount of data that shows beneficial effects of SCFA on control of body weight and insulin sensitivity ${ }^{29,61}$.

In particular since fecal metabolite concentrations are the net result of various factors including whole gut transit time, gut microbiota composition, microbial cross-feeding mechanisms, complex microbial interactions as well as colonic rates of absorption that may differ along the colon (proximal versus distal) and are not necessarily a good reflection of SCFA production rates ${ }^{69}$.

More in detail, in our analysis (chapter 4) circulating SCFA (acetate, propionate and butyrate) associated negatively with circulating lipids (glycerol, triacyclglycerols and free fatty acids, respectively). Of note, in this study the antilipolytic effect of acetate was corroborated as previous in vitro ${ }^{35-37,39}$, animal ${ }^{35}$, ${ }^{38}$ and human studies have reported ${ }^{40-43}$. Mechanistically, the antilipolytic effect of acetate may be mediated through a G-protein coupled receptor (GPR)-mediated decrease in hormone sensitive lipase phosphorylation in human adipocytes as observed in chapter 7 . The negative association between propionate with triacylglycerols may be explained through an increase in lipoprotein lipase in the adipose tissue as observed in vitro ${ }^{70}$, which may increase lipid extraction from the circulation. With respect to butyrate, data is inconsistent as in vitro studies have reported both anti- and pro-lipolytic effects in white adipose tissue ${ }^{39,71}$.

In addition, in our analysis in chapter 4 butyrate showed a negative correlation with glucose levels, which is in line with rodent studies in which oral butyrate supplementation improved insulin sensitivity ${ }^{30,72}$. However, butyrate effects in humans may depend on metabolic phenotype, as already explained above, since an oral butyrate supplementation improved glucose metabolism in lean but not in individuals with metabolic syndrome ${ }^{27}$. In addition microbial phenotype may play a regulatory role as well, as it has been consistently reported that insulin resistant/diabetic individuals have low numbers of butyrateproducing bacteria ${ }^{73-75}$. Further studies are warranted to elucidate to what extent the SCFA production and handling is disturbed in metabolically compromised individuals.

In the colon, human enteroendocrine $\mathrm{L}$ cells have reported to secrete gut hormones (e. g. GLP-1, PYY) through SCFA stimulation of GPR41/43 receptors ${ }^{76}$, 77. Interestingly, we found that circulating SCFA were positively associated with fasting GLP-1. It is important to interpret this considering that residual (slowfermenting) dietary fibers may still be fermented in the colon and that whole gut transit time may take longer than an usual overnight fast ${ }^{78}$. To our knowledge this is the first study that reports an association of circulating SCFA with GLP-1 in the fasted state. In rodents, previous studies have reported increments in gut hormones after dietary fiber supplementation in the postprandial state ${ }^{79}$. 
Circulating SCFA may stimulate GLP-1 secretion from the visceral, basolateral side of enteroendocrine L-cells, as observed in a study using isolated rat colons ${ }^{80}$. Of note, the stimulus was rather weak, thus questioning the physiological relevance. Besides enteroendocrine L-cells, pancreatic $\alpha$-cells have been suggested to contribute to systemic GLP-1 concentrations in the fasted state, but whether circulating SCFA act as stimuli for GLP-1 secretion warrants further investigation 81.

\section{Circulating acetate and propionate in relation to insulin sensitivity}

In a subset of the cohort in chapter 4, we investigated the associations of fecal/plasma SCFA with insulin sensitivity as measured via the gold standard hyperinsulinaemic-euglycemic clamp technique. Interestingly, we found that circulating acetate associated negatively (similarly to the above findings), while circulating propionate associated positively with insulin sensitivity. The reason why we did not observe a sexual dimorphism in the analysis in chapter 4 is not entirely clear and remains to be clarified. A factor which may play a role in explaining the differences between studies is the metabolic phenotype of the individuals since a larger proportion of the participants in chapter 4 had an impaired glucose metabolism.

Indeed, a recent study from the DiOGenes consortium and the Maastricht Study reported that obese (not diabetic) individuals with hepatic insulin resistance may have decreased ketogenesis ${ }^{82}$, and possibly suggesting a differential release of acetate from endogenous sources into the circulation. Thus, the finding of an inverse correlation of circulating acetate with insulin sensitivity is in contrast to vast amount of data in animal studies 31,32, 83-88, human interventions 28, 44, 45, and association studies ${ }^{46,47}$ that report positive effects on insulin sensitivity. The exact mechanisms for these discrepancies remain to be elucidated, but may relate to metabolic condition (fasting, energy restriction), sex differences as well as metabolic phenotype. In line with our positive relationship of propionate and insulin sensitivity in chapter 4 , a human study showed that oral administration of sodium propionate increased fasting fat oxidation and resting energy expenditure in healthy subjects ${ }^{34}$. Mechanistically, propionate may attenuate intrahepatocellular lipids, increase glycogen synthesis and decreased inflammatory markers ${ }^{89}, 90$. Overall, circulating SCFA may reflect more accurately metabolic health parameters (fasting GLP-1, circulating lipids and insulin sensitivity) as compared to fecal levels. This suggests that the measurement of circulating SCFA is a requirement when investigating the role of the gut microbiome in metabolic health.

Nevertheless, studies investigating SCFA dynamics are needed to provide a better insight of SCFA colonic production, absorption and how circulating levels may impact the function and metabolism of peripheral tissues. 
In the following paragraph, we discuss how SCFA might impact skeletal muscle and adipose tissue substrate metabolism.

\section{Mechanisms of action of SCFA on skeletal muscle and adipose tissue metabolism}

According to the literature, SCFA may exert their whole-body effects through effects on various tissues including skeletal muscle and adipose tissue metabolism. Of note, SCFA may be the link between gut and skeletal muscle and adipose tissue glucose homeostasis and lipid metabolism ${ }^{29,91}$. For instance, in previous in vivo human studies distal colonic SCFA infusions (rich in acetate) increased whole-body fasting fat oxidation in men with overweight/obesity ${ }^{45,48}$. Similarly, oral administration of sodium propionate increased fasting fat oxidation and resting energy expenditure independent of glucose and insulin levels in healthy subjects ${ }^{34}$. With respect to skeletal muscle metabolism, acetate has been reported to have the potential to increase the oxidative capacity through increments in AMP/ATP ratio, AMPK phosphorylation and upregulation of GLUT4 ${ }^{31}$ 32. In line muscle gastrocnemius extracts from butyrate supplemented mice (C57BL/6J) showed increments in radiolabeled palmitate oxidation by $200 \%$ 30. Furthermore, in relation to adipose tissue metabolism, in vitro, animal and human studies have reported antilipolytic effects in response to acetate ${ }^{35-43}$. Importantly, in vitro mechanistic studies have been performed mainly in murine adipose cells (which may lack GPR41) ${ }^{92}$ with incubations of single SCFA using supraphysiological concentrations $(4-10 \mathrm{mmol} / \mathrm{L})$. Based on previous findings, we hypothesized that circulating acetate may directly affect fat oxidation in human skeletal muscle contributing to the observed increments in in vivo whole-body fat oxidation.

\section{Effect of acetate on fat oxidation in human primary muscle cells}

In chapter 6, we investigated in vitro, the dose and time effect of sodium acetate on complete and incomplete endogenous and exogenous oxidation of ${ }^{14} \mathrm{C}$ labelled palmitate. In detail, we investigated the effects of acetate in putatively physiological concentrations $(0-0.5 \mathrm{mmol} / \mathrm{l})$ reached after fiber fermentation ${ }^{93-96}$ as well as supraphysiological concentrations (1-5 mmol/l). Although, intracellular/interstitial concentration of acetate in skeletal muscle remains unknown, we used a wide spectrum of concentrations, thus likely mimicking in vivo cellular and extracellular concentrations after fiber fermentation. As shown in chapter 6, our in vitro data showed no effect of sodium acetate on neither complete nor incomplete endogenous and exogenous oxidation, irrespective of time and dose in human primary muscle cells (HSkMC). 
In addition, we investigated the potential effect of sodium acetate on AMPactivated protein kinase (AMPK) phosphorylation in our HSkMC model. In line with our in vitro fat oxidation data, we could not observe an effect of sodium acetate on total and phosphorylated AMPK protein content in our HSkMC model.

In contrast, in rat L6 myotubes, acetate increased AMP/ATP ratio and AMPK (threonine 172) phosphorylation in a dose-dependent $(0.05-0.5 \mathrm{mmol} / \mathrm{L})$ and time-dependent $(0-30 \mathrm{~min})$ manner ${ }^{31}$. In addition to these in vitro observations, oral injection of acetate acutely increased phosphorylation of Thr172-AMPK $\alpha$, in rat skeletal muscle (in vivo) ${ }^{31,32}$.

Furthermore, it needs to be mentioned that AMPK-dependent fat oxidation might be limited in HSkMC and/or an AMPK-dependent effect on glucose uptake/oxidation may be preferentially activated in human skeletal muscle cells ${ }^{97}$, 98. Although acetate increases AMPK phosphorylation in rodents, acetatemediated effect on AMPK activation in skeletal muscle may be species-specific ${ }^{99}$ as well as muscle fiber type dependent, since AMPK subunits may differ between muscle type fibers ${ }^{100}$. Of importance, the higher complexity of the in vivo situations and the interorgan crosstalk with other tissues may partly explain the lack of effect in our in vitro conditions as compared to our previous human interventions ${ }^{45}$. For instance, sodium acetate may increase oxidative capacity in other tissues, as in vitro and in vivo rodent studies have shown increments in phosphorylated AMPK and total AMPK in the colon, liver and adipose tissue ${ }^{32,33,86}$. Secondly, other SCFA may increase fat oxidation in vivo, as discussed above in healthy individuals after oral administration of sodium propionate ${ }^{34}$. Finally, the previously observed increment in fat oxidation after SCFA infusion may be partly explained by the ability of these SCFA to stimulate the secretion of other gut-derived hormones such as PYY and GLP-1, which have been associated with increased whole-body fat oxidation and energy expenditure in humans ${ }^{101,102}$. Furthermore, fat oxidation might be donor-dependent, as individual metabolic characteristics of donors have been reported to be retained in in vitro HSkMC models ${ }^{103,104}$. Considering that we used muscle cells derived from an insulin sensitive donor and the in vivo differences between metabolic phenotypes, it remains a challenge to investigate further donor differences at the in vitro level as well as intrinsic donor characteristics such as sex, age, diet. In summary, further studies may focus on other metabolic players such as propionate or butyrate, and/or SCFA mixtures and gut hormones as well as investigate the oxidative capacity of other tissues (i.e. liver, adipose tissue) in response to SCFA.

Nevertheless, this is to our knowledge the first study that investigates the effects of the most abundant SCFA acetate on fat oxidation in a HSkMC model. 


\section{Effect of SCFA on lipolysis in human adipocytes}

As discussed above with respect to adipose tissue metabolism, several studies have reported antilipolytic effects of acetate in vivo. Of importance, this is in line with findings in chapter 4, that reported that circulating SCFA (acetate, propionate and butyrate) were negatively associated with fasting circulating lipids (glycerol, triacyclglycerols and free fatty acids, respectively). Therefore, in chapter 7, we investigated the in vitro antilipolytic properties of single and combinations of SCFA at physiological $(1 \mu \mathrm{mol} / \mathrm{l})$ and supraphysiological $(1 \mathrm{mmol} / \mathrm{l})$ concentrations in human multipotent adipose tissue derived stem (hMADS) cells. Interestingly, we showed that SCFA mixtures high in acetate and propionate decreased basal glycerol release, when compared to control, while mixtures high in butyrate had no effect. $\beta$-adrenergic receptor mediated glycerol release was not significantly altered following incubation with SCFA mixtures. However, incubation with only acetate decreased both basal $(1 \mu \mathrm{mol} / \mathrm{L})$ and $\beta$-adrenergically $(1 \mu \mathrm{mol} / \mathrm{L}$ and $1 \mathrm{mmol} / \mathrm{L})$ mediated glycerol release when compared with control treated cells. In contrast, butyrate $(1 \mu \mathrm{mol} / \mathrm{L})$ slightly increased basal and $\beta$ adrenergically mediated glycerol release compared with control, while propionate had no effect on lipolysis. Of interest, the antilipolytic effect of acetate was accompanied by a reduced serine phosphorylation of the major cytosolic lipase hormone-sensitive lipase (HSL). In addition, inhibition of inhibitory G proteins following pertussis toxin treatment prevented the antilipolytic effect of acetate.

Taken together, our data shows that acetate, the most abundant SCFA in the colon and in the circulation $(19-450 \mu \mathrm{mol} / \mathrm{L})$, is the main driver of GPRdependent antilipolytic effect of SCFA in human adipocytes ${ }^{29,66}$. Even though a study in murine 3T3-L1 adipocytes $(4 \mathrm{mmol} / \mathrm{l}){ }^{36}$ and human primary adipocytes $(10 \mathrm{mmol} / \mathrm{l}){ }^{37}$ showed antilipolytic effect using physiological concentrations of acetate $(100-300 \mu \mathrm{mol} / \mathrm{l})$, it is important to consider that animal cells may lack the major acetate GPR, GPR41 ${ }^{92}$, thus might not capture the human complexity. In addition, it needs to be mentioned that we used pooled hMADS from different donors with multiple metabolic characteristics. It would be worthwhile to investigate the antilipolytic potential of acetate in adipocytes from insulin resistant compared to insulin sensitive donors and whether this is translated to the in vivo situation. Finally, future studies on the microbiome-host metabolism interactions (pre, pro and post-biotics) have to further elucidate the role of SCFA (i.e. acetate) in adipose tissue and skeletal muscle metabolism using stable isotope tracer for studying SCFA dynamics and elucidating pathways in detail in tissue biopsies. 


\section{Microbial metabolites derived from proteolytic fermentation and insulin sensitivity}

Besides saccharolytic, proteolytic fermentation may occur mainly in the distal colon and produce a wide variety of metabolites including phenolic compounds, indoles, hydrogen sulfide as well as BCFA such as isovalerate and isobutyrate ${ }^{11-13}$. In general, high proteolytic fermentation elicited by high protein diets (HPD), a relatively high content of plant-based proteins, or a diet low in indigestible carbohydrates may lead to altered production of microbial derived metabolites (i.e. increase fecal BCFA and decrease fecal SCFA), alterations in gut microbiota, colonic epithelium as well as impaired detoxification capacity of gut mucosa and increased inflammation ${ }^{105}$. In addition, as extensively discussed, most research focuses on the major saccharolytic byproducts SCFA acetate, propionate and butyrate, and metabolic roles of other saccharolytic fermentation byproducts (i.e. caproate, lactate) remain largely unexplored. Therefore, in chapter 5 we investigated the associations of fecal BCFA (isovalerate, isobutyrate) as well as other fecal metabolites (valerate, caproate, succinate and lactate) with parameters of metabolic health in a cohort of 88 participants with a wide range of BMI and glucometabolic status. We did not find associations of fecal metabolites with the metabolic markers as well as no differences with respect to the degree of insulin resistance. These findings are in line with a study that did not find differences in fecal BCFA levels between overweight/obese and healthy individuals ${ }^{67}$. In contrast, elevated fecal BCFA concentrations have been observed in obese ${ }^{106}$ and hypercholesterolemic individuals ${ }^{16}$ as well as BCFA have been associated to NAFLD progression ${ }^{15}$. Moreover, obese individuals have been reported to have lower circulating isobutyrate and higher isovalerate concentrations as compared to healthy individuals ${ }^{107}$. On the tissue level, an in vitro study using rat and human adipocytes showed that both BCFA (isobutyrate and isovalerate) inhibited cytosolic (intracellular) lipolysis and lipogenesis using supraphysiological concentrations $(10 \mathrm{mmol} / \mathrm{L})^{37}$. Nevertheless, in our study we could not confirm any differences in fecal BCFA concentrations, which partly reflect BCFA production, between insulin resistant and insulin sensitive individuals.

However, as already indicated above, it is important to mention that fecal metabolites depend on various factors including microbial community 108 , intestinal transit time ${ }^{69}$, diet ${ }^{109,110}$, age ${ }^{111}$, lifestyle ${ }^{112}$ among others. Whether gutderived metabolites need to enter the circulation to affect host metabolic health as previously suggested for SCFA ${ }^{66}$ warrants further investigation, in particular considering that BCFA are mainly produced in the distal colon. In conclusion, the present data do not show any correlation in proteolytic fermentation metabolites (fecal BCFA) and the saccharolytic metabolites lactate, succinate, valerate and caproate with degree of insulin resistance. 
Future studies with the aim to modulate saccharolytic/proteolytic fermentation should measure the kinetics of these metabolites as well as plasma concentrations taking into account the metabolic ${ }^{26-28}$ and microbial phenotypes ${ }^{17-}$ 19.

\section{The role of metabolic phenotype in dietary intervention outcome}

It is increasingly evident that microbial and metabolic phenotype should be considered as nutritional interventions may depend on microbial enterotypes (Bacteroides, Prevotella, Ruminococcus) ${ }^{17}$ or metabolic phenotypes ${ }^{55}$. As discussed above, prebiotic and vinegar interventions have shown beneficial effects on glucose control in lean, but to a lesser extent in individuals with an impaired glucose homeostasis ${ }^{28,113}$. Additionally, we observed a sexual dimorphism in the relationship between acetate and insulin sensitivity in the analysis of the DiOGenes study (chapter 3, as discussed above), which warrants further research. Furthermore, restoring gut microbiota composition using probiotics (i.e. Akkermansia), its nutrients (prebiotics), byproducts (postbiotics) and/or combinations (synbiotics) considering colonic site might be of key importance for metabolically compromised individuals in terms of restoring metabolic health. In summary, future nutritional interventions may need to consider tissue specific metabolism as well as microbial phenotype (composition and functionality) to develop targeted intervention strategies in order to optimize metabolic health outcome ${ }^{114-116}$.

\section{Conclusion and future perspectives}

This doctoral thesis focuses on the role of the gut microbially-derived metabolites SCFA as well as BCFA in human substrate and energy metabolism. To study in vivo the metabolic effects and underlying mechanisms, we combined association studies in individuals with a broad range of BMI and glucometabolic status, with in vitro mechanistic experiments using stem cells derived from human adipose tissue and skeletal muscle biopsies.

We found that fasting circulating SCFA were associated with metabolic health markers (fasting GLP-1, circulating lipids) and insulin sensitivity in human cohorts. In contrast to previous findings we found that circulating acetate was negatively associated with insulin sensitivity. Additionally, there was a sexual dimorphism in the relationship between acetate and insulin sensitivity in analysis in the DiOGenes study. Further studies on the colonic and endogenous sources responsible for acetate production and acetate clearance are required in different metabolic phenotypes. 
Furthermore, while acetate is the major SCFA for the pronounced GPRdependent antilipolytic effect in human adipocytes, there were no acetatemediated effects on fat oxidation in our human skeletal muscle cell model.

The main outcomes of this thesis provide important insight in the role of SCFA and BCFA in human substrate and energy metabolism. However, future studies investigating the metabolic effects of microbial metabolites should consider various aspects including:

1. Metabolic phenotype as it may possibly affect SCFA and BCFA handling of the host. In this regard, it has been reported that tissue specific metabolism and insulin resistance or microbial phenotype may affect intervention outcome ${ }^{55,82}$. It would be highly valuable to elucidate to what extent the balance between saccharolytic and proteolytic fermentation (SCFA and BCFA production) and in particular the site of colonic fermentation (proximal vs distal) may affect liver and/or skeletal muscle insulin resistance. Additionally, human studies are lacking investigating SCFA/BCFA dynamics as well as the effect of circulating BCFA, especially derived from proteolytic (animal products and plant based) fermentation on tissue metabolism and whole-body homeostasis.

2. Sexual dimorphism. In chapter 3, we describe that fasting acetate associated positively with insulin resistance in females, not in males. Of note, various studies have reported that gut microbial diversity is linked to insulin sensitivity ${ }^{20-25}$ and in a sex-specific manner ${ }^{117}$. Further studies should investigate our sex-specific findings, possibly by elucidating whether the contribution of gut-derived acetate to fasting circulating acetate may be different in males and females. This may provide a better understanding of how circulating acetate may affect metabolism. Additionally, the difference in associations of acetate and propionate as well as the propionate/acetate ratio with insulin sensitivity should be further investigated.

3. Fecal versus circulating levels. In chapter 4 , we show that circulating, but not fecal, SCFA are linked to circulating GLP-1 concentrations, whole-body lipolysis and peripheral insulin sensitivity in humans. It is of great interest to elucidate whether plasma SCFA may induce GLP-1 secretion in other tissues such as the alpha cells in the pancreas.

4. Further studies on the colonic and endogenous sources responsible for acetate production and acetate clearance are required in different metabolic phenotypes. In addition, with emphasis on skeletal muscle, liver and pancreas to investigate tissue specific effects of acetate.

5. In vitro mechanistic studies. In chapter 6 and chapter 7, we provide valuable data on the possible mechanism of action of SCFA in human 
derived primary cells models. However, technically challenging and demanding gain and loss-of function experiments in this primary cell model are needed to elucidate the exact intracellular mode of action. Further in vitro studies in skeletal muscle cell models should also consider donor characteristics, fiber type dependent AMPK activation as well as SCFA mixtures to mimic an in vivo postprandial situation. In addition, future in vitro studies may need to consider comparing cell models derived from donors of different metabolic phenotypes. Furthermore, to investigate effects of gut-altering interventions at the tissue level, muscle and adipose tissue biopsies may provide key information on pathways involved in SCFA handling and their putative metabolic effects. 


\section{References}

1. Engin A. The Definition and Prevalence of Obesity and Metabolic Syndrome. Adv Exp Med Biol. 2017;960:1-17. Epub 2017/06/07. doi: 10.1007/978-3319-48382-5_1. PubMed PMID: 28585193.

2. Snel M, Jonker JT, Schoones J, Lamb H, de Roos A, Pijl H, et al. Ectopic fat and insulin resistance: pathophysiology and effect of diet and lifestyle interventions. Int J Endocrinol. 2012;2012:983814. Epub 2012/06/08. doi: 10.1155/2012/983814. PubMed PMID: 22675355; PubMed Central PMCID: РМСРМС3366269.

3. Makki K, Froguel P, Wolowczuk I. Adipose tissue in obesity-related inflammation and insulin resistance: cells, cytokines, and chemokines. ISRN Inflamm. 2013;2013:139239. Epub 2014/01/24. doi: 10.1155/2013/139239. PubMed PMID: 24455420; PubMed Central PMCID: PMCPMC3881510.

4. Gastaldelli A, Gaggini M, DeFronzo RA. Role of Adipose Tissue Insulin Resistance in the Natural History of Type 2 Diabetes: Results From the San Antonio Metabolism Study. Diabetes. 2017;66(4):815. doi: 10.2337/db16-1167.

5. Goossens GH, Blaak EE, Theunissen R, Duijvestijn AM, Clément K, Tervaert JW, et al. Expression of NLRP3 inflammasome and T cell population markers in adipose tissue are associated with insulin resistance and impaired glucose metabolism in humans. Mol Immunol. 2012;50(3):142-9. Epub 2012/02/14. doi: 10.1016/j.molimm.2012.01.005. PubMed PMID: 22325453.

6. Meldrum DR, Morris MA, Gambone JC. Obesity pandemic: causes, consequences, and solutions-but do we have the will? Fertil Steril. 2017;107(4):8339. Epub 2017/03/16. doi: 10.1016/j.fertnstert.2017.02.104. PubMed PMID: 28292617. 7. Lara-Castro C, Garvey WT. Intracellular lipid accumulation in liver and muscle and the insulin resistance syndrome. Endocrinol Metab Clin North Am. 2008;37(4):841-56. doi: 10.1016/j.ecl.2008.09.002. PubMed PMID: 19026935.

8. Sanders ME, Merenstein DJ, Reid G, Gibson GR, Rastall RA. Probiotics and prebiotics in intestinal health and disease: from biology to the clinic. Nat Rev Gastroenterol Hepatol. 2019. Epub 2019/07/13. doi: 10.1038/s41575-019-0173-3. PubMed PMID: 31296969.

9. Canfora EE, Jocken JW, Blaak EE. Short-chain fatty acids in control of body weight and insulin sensitivity. Nat Rev Endocrinol. 2015;11(10):577-91. doi: 10.1038/nrendo.2015.128.

10. Koh A, De Vadder F, Kovatcheva-Datchary P, Bäckhed F. From Dietary Fiber to Host Physiology: Short-Chain Fatty Acids as Key Bacterial Metabolites. Cell. 2016;165(6):1332-45. doi: 10.1016/j.cell.2016.05.041.

11. Mika A, Stepnowski P, Kaska L, Proczko M, Wisniewski P, Sledzinski M, et al. A comprehensive study of serum odd- and branched-chain fatty acids in patients with excess weight. Obesity (Silver Spring). 2016;24(8):1669-76. Epub 2016/06/30. doi: 10.1002/oby.21560. PubMed PMID: 27355152. 
12. Canfora EE, Meex RCR, Venema K, Blaak EE. Gut microbial metabolites in obesity, NAFLD and T2DM. Nature Reviews Endocrinology. 2019;15(5):261-73. doi: 10.1038/s41574-019-0156-z.

13. Ran-Ressler RR, Bae S, Lawrence P, Wang DH, Brenna JT. Branched-chain fatty acid content of foods and estimated intake in the USA. Br J Nutr. 2014;112(4):565-72. Epub 2014/05/17. doi: 10.1017/s0007114514001081. PubMed PMID: 24830474; PubMed Central PMCID: PMCPMC4381348.

14. Le Roy $\mathrm{T}$, Llopis M, Lepage $\mathrm{P}$, Bruneau A, Rabot S, Bevilacqua C, et al. Intestinal microbiota determines development of non-alcoholic fatty liver disease in mice. Gut. 2013;62(12):1787-94. Epub 2012/12/01. doi: 10.1136/gutjnl-2012303816. PubMed PMID: 23197411.

15. Da Silva HE, Teterina A, Comelli EM, Taibi A, Arendt BM, Fischer SE, et al. Nonalcoholic fatty liver disease is associated with dysbiosis independent of body mass index and insulin resistance. Sci Rep. 2018;8(1):1466. Epub 2018/01/25. doi: 10.1038/s41598-018-19753-9. PubMed PMID: 29362454; PubMed Central PMCID: PMCPMC5780381.

16. Granado-Serrano AB, Martín-Garí M, Sánchez V, Riart Solans M, Berdún R, Ludwig IA, et al. Faecal bacterial and short-chain fatty acids signature in hypercholesterolemia. Scientific Reports. 2019;9(1):1772. doi: 10.1038/s41598-01938874-3.

17. Arumugam M, Raes J, Pelletier E, Le Paslier D, Yamada T, Mende DR, et al. Enterotypes of the human gut microbiome. Nature. 2011;473(7346):174-80. doi: 10.1038/nature09944.

18. Kovatcheva-Datchary P, Nilsson A, Akrami R, Lee YS, De Vadder F, Arora $\mathrm{T}$, et al. Dietary Fiber-Induced Improvement in Glucose Metabolism Is Associated with Increased Abundance of Prevotella. Cell metabolism. 2015;22(6):971-82. Epub 2015/11/11. doi: 10.1016/j.cmet.2015.10.001. PubMed PMID: 26552345.

19. Holscher HD. Dietary fiber and prebiotics and the gastrointestinal microbiota. Gut Microbes. 2017;8(2):172-84. Epub 2017/02/06. doi: 10.1080/19490976.2017.1290756. PubMed PMID: 28165863.

20. Koliada A, Syzenko G, Moseiko V, Budovska L, Puchkov K, Perederiy V, et al. Association between body mass index and Firmicutes/Bacteroidetes ratio in an adult Ukrainian population. BMC Microbiol. 2017;17(1):120-. doi: 10.1186/s12866017-1027-1. PubMed PMID: 28532414.

21. Turnbaugh PJ, Ley RE, Mahowald MA, Magrini V, Mardis ER, Gordon JI. An obesity-associated gut microbiome with increased capacity for energy harvest. Nature. 2006;444(7122):1027-31. doi: 10.1038/nature05414.

22. Ley RE, Bäckhed F, Turnbaugh P, Lozupone CA, Knight RD, Gordon JI. Obesity alters gut microbial ecology. Proceedings of the National Academy of Sciences of the United States of America. 2005;102(31):11070. doi: 10.1073/pnas.0504978102. 
23. Castaner O, Goday A, Park Y-M, Lee S-H, Magkos F, Shiow S-ATE, et al. The Gut Microbiome Profile in Obesity: A Systematic Review. Int J Endocrinol. 2018;2018:4095789-. doi: 10.1155/2018/4095789. PubMed PMID: 29849617.

24. Mariat D, Firmesse O, Levenez F, Guimarăes VD, Sokol H, Doré J, et al. The Firmicutes/Bacteroidetes ratio of the human microbiota changes with age. BMC Microbiol. 2009;9(1):123. doi: 10.1186/1471-2180-9-123.

25. Schwiertz A, Taras D, Schäfer K, Beijer S, Bos NA, Donus C, et al. Microbiota and SCFA in Lean and Overweight Healthy Subjects. Obesity. 2010;18(1):190-5. doi: 10.1038/oby.2009.167.

26. Hansen AMB, Wium C, Lee S, Tierney AC, McCarthy D, Roche HM, et al. Substantial inter-individual variations in insulin secretion and sensitivity across the glucometabolic spectrum. Scandinavian Journal of Clinical and Laboratory Investigation. 2020:1-9. doi: 10.1080/00365513.2020.1730433.

27. Bouter K, Bakker GJ, Levin E, Hartstra AV, Kootte RS, Udayappan SD, et al. Differential metabolic effects of oral butyrate treatment in lean versus metabolic syndrome subjects. Clin Transl Gastroenterol. 2018;9(5):155-. doi: 10.1038/s41424018-0025-4. PubMed PMID: 29799027.

28. Lim J, Henry CJ, Haldar S. Vinegar as a functional ingredient to improve postprandial glycemic control-human intervention findings and molecular mechanisms. Mol Nutr Food Res. 2016;60(8):1837-49. Epub 2016/05/24. doi: 10.1002/mnfr.201600121. PubMed PMID: 27213723.

29. Canfora EE, Jocken JW, Blaak EE. Short-chain fatty acids in control of body weight and insulin sensitivity. Nat Rev Endocrinol. 2015;11(10):577-91. Epub 2015/08/12. doi: 10.1038/nrendo.2015.128. PubMed PMID: 26260141.

30. Gao Z, Yin J, Zhang J, Ward RE, Martin RJ, Lefevre M, et al. Butyrate Improves Insulin Sensitivity and Increases Energy Expenditure in Mice. Diabetes. 2009;58(7):1509. doi: 10.2337/db08-1637.

31. Maruta H, Yoshimura Y, Araki A, Kimoto M, Takahashi Y, Yamashita H. Activation of AMP-Activated Protein Kinase and Stimulation of Energy Metabolism by Acetic Acid in L6 Myotube Cells. PLoS One. 2016;11(6):e0158055. doi: 10.1371/journal.pone.0158055.

32. Yamashita H, Maruta H, Jozuka M, Kimura R, Iwabuchi H, Yamato M, et al. Effects of acetate on lipid metabolism in muscles and adipose tissues of type 2 diabetic Otsuka Long-Evans Tokushima Fatty (OLETF) rats. Biosci Biotechnol Biochem. 2009;73(3):570-6. Epub 2009/03/10. doi: 10.1271/bbb.80634. PubMed PMID: 19270372.

33. Sakakibara S, Yamauchi T, Oshima Y, Tsukamoto Y, Kadowaki T. Acetic acid activates hepatic AMPK and reduces hyperglycemia in diabetic KK-A(y) mice. Biochemical and Biophysical Research Communications. 2006;344(2):597604. doi: 10.1016/j.bbrc.2006.03.176.

34. Chambers ES, Byrne CS, Aspey K, Chen Y, Khan S, Morrison DJ, et al. Acute oral sodium propionate supplementation raises resting energy expenditure and lipid oxidation in fasted humans. Diabetes Obes Metab. 2018;20(4):1034-9. Epub 
2017/11/15. doi: 10.1111/dom.13159. PubMed PMID: 29134744; PubMed Central PMCID: PMCPMC5873405.

35. Ge H, Li X, Weiszmann J, Wang P, Baribault H, Chen J-L, et al. Activation of G Protein-Coupled Receptor 43 in Adipocytes Leads to Inhibition of Lipolysis and Suppression of Plasma Free Fatty Acids. Endocrinology. 2008;149(9):4519-26. doi: 10.1210/en.2008-0059.

36. Aberdein N, Schweizer M, Ball D. Sodium acetate decreases phosphorylation of hormone sensitive lipase in isoproterenol-stimulated 3T3-L1 mature adipocytes. Adipocyte. 2014;3(2):121-5. doi: 10.4161/adip.27936. PubMed PMID: PMC3979876.

37. Heimann E, Nyman M, Pålbrink A-K, Lindkvist-Petersson K, Degerman E. Branched short-chain fatty acids modulate glucose and lipid metabolism in primary adipocytes. Adipocyte. 2016;5(4):359-68. doi: 10.1080/21623945.2016.1252011. PubMed PMID: 27994949.

38. Sahuri-Arisoylu M, Brody LP, Parkinson JR, Parkes H, Navaratnam N, Miller AD, et al. Reprogramming of hepatic fat accumulation and 'browning' of adipose tissue by the short-chain fatty acid acetate. International journal of obesity (2005). 2016;40(6):955-63. Epub 2016/03/16. doi: 10.1038/ijo.2016.23. PubMed PMID: 26975441.

39. Jocken JWE, González Hernández MA, Hoebers NTH, van der Beek CM, Essers YPG, Blaak EE, et al. Short-Chain Fatty Acids Differentially Affect Intracellular Lipolysis in a Human White Adipocyte Model. Front Endocrinol (Lausanne). 2018;8(372). doi: 10.3389/fendo.2017.00372.

40. Crouse JR, Gerson CD, DeCarli LM, Lieber CS. Role of acetate in the reduction of plasma free fatty acids produced by ethanol in man. J Lipid Res. 1968;9(4):509-12.

41. Laurent C, Simoneau C, Marks L, Braschi S, Champ M, Charbonnel B, et al. Effect of acetate and propionate on fasting hepatic glucose production in humans. Eur J Clin Nutr. 1995;49(7):484-91. Epub 1995/07/01. PubMed PMID: 7588498.

42. Wolever TM, Brighenti F, Royall D, Jenkins AL, Jenkins DJ. Effect of rectal infusion of short chain fatty acids in human subjects. Am J Gastroenterol. 1989;84(9):1027-33. Epub 1989/09/01. PubMed PMID: 2773895.

43. Suokas A, Kupari M, Heikkila J, Lindros K, Ylikahri R. Acute cardiovascular and metabolic effects of acetate in men. Alcoholism, clinical and experimental research. 1988;12(1):52-8. Epub 1988/02/01. PubMed PMID: 3279860. 44. Freeland KR, Wolever TMS. Acute effects of intravenous and rectal acetate on glucagon-like peptide-1, peptide $Y Y$, ghrelin, adiponectin and tumour necrosis factor-alpha. The British journal of nutrition. 2010;103(3):460-6. doi: 10.1017/s0007114509991863. PubMed PMID: 19818198.

45. van der Beek CM, Canfora EE, Lenaerts K, Troost FJ, Damink S, Holst JJ, et al. Distal, not proximal, colonic acetate infusions promote fat oxidation and improve metabolic markers in overweight/obese men. Clin Sci (Lond). 
2016;130(22):2073-82. Epub 2016/07/22. doi: 10.1042/cs20160263. PubMed PMID: 27439969.

46. Layden BT, Yalamanchi SK, Wolever TM, Dunaif A, Lowe WL, Jr. Negative association of acetate with visceral adipose tissue and insulin levels. Diabetes, metabolic syndrome and obesity : targets and therapy. 2012;5:49-55. Epub 2012/02/28. doi: 10.2147/DMSO.S29244. PubMed PMID: 22419881.

47. Moreno-Navarrete JM, Serino M, Blasco-Baque V, Azalbert V, Barton RH, Cardellini M, et al. Gut Microbiota Interacts with Markers of Adipose Tissue Browning, Insulin Action and Plasma Acetate in Morbid Obesity. Molecular Nutrition \& Food Research. 2018;62(3):1700721. doi: 10.1002/mnfr.201700721.

48. Canfora EE, van der Beek CM, Jocken JWE, Goossens GH, Holst JJ, Olde Damink SWM, et al. Colonic infusions of short-chain fatty acid mixtures promote energy metabolism in overweight/obese men: a randomized crossover trial. Scientific Reports. 2017;7(1):2360. doi: 10.1038/s41598-017-02546-x.

49. Neis EPJG, van Eijk HMH, Lenaerts K, Olde Damink SWM, Blaak EE, Dejong $\mathrm{CHC}$, et al. Distal versus proximal intestinal short-chain fatty acid release in man. Gut. 2019;68(4):764. doi: 10.1136/gutjnl-2018-316161.

50. Canfora EE, van der Beek CM, Hermes GDA, Goossens GH, Jocken JWE, Holst JJ, et al. Supplementation of Diet With Galacto-oligosaccharides Increases Bifidobacteria, but Not Insulin Sensitivity, in Obese Prediabetic Individuals. Gastroenterology. 2017;153(1):87-97.e3. Epub 2017/04/12. doi: 10.1053/j.gastro.2017.03.051. PubMed PMID: 28396144.

51. Canfora EE MM, Hermes G et al. A novel fiber mixture to promote microbial fermentation in the distal colon affects substrate metabolism in lean vs prediabetic obese men. Obes Facts 12(suppl 1), 191. 2019.

52. Zhao L, Zhang F, Ding X, Wu G, Lam YY, Wang X, et al. Gut bacteria selectively promoted by dietary fibers alleviate type 2 diabetes. Science. 2018;359(6380):1151-6. Epub 2018/03/29. doi: 10.1126/science.aao5774. PubMed PMID: 29590046.

53. Chambers ES, Byrne CS, Morrison DJ, Murphy KG, Preston T, Tedford C, et al. Dietary supplementation with inulin-propionate ester or inulin improves insulin sensitivity in adults with overweight and obesity with distinct effects on the gut microbiota, plasma metabolome and systemic inflammatory responses: a randomised cross-over trial. Gut. 2019;68(8):1430. doi: 10.1136/gutjnl-2019-318424. 54. Wu GD, Chen J, Hoffmann C, Bittinger K, Chen Y-Y, Keilbaugh SA, et al. Linking Long-Term Dietary Patterns with Gut Microbial Enterotypes. Science. 2011;334(6052):105. doi: 10.1126/science.1208344.

55. Blaak EE. Current metabolic perspective on malnutrition in obesity: towards more subgroup-based nutritional approaches? Proceedings of the Nutrition Society. 2020:1-7. Epub 2020/03/03. doi: 10.1017/S0029665120000117.

56. Tenorio-Jiménez C, Martínez-Ramírez MJ, Gil Á, Gómez-Llorente C. Effects of Probiotics on Metabolic Syndrome: A Systematic Review of Randomized Clinical Trials. Nutrients. 2020;12(1):124. PubMed PMID: doi:10.3390/nu12010124. 
57. Ejtahed H-S, Angoorani P, Soroush A-R, Atlasi R, Hasani-Ranjbar S, Mortazavian AM, et al. Probiotics supplementation for the obesity management; A systematic review of animal studies and clinical trials. Journal of Functional Foods. 2019;52:228-42. doi: 10.1016/j.jff.2018.10.039.

58. Xu Y, Wang N, Tan H-Y, Li S, Zhang C, Feng Y. Function of Akkermansia muciniphila in Obesity: Interactions With Lipid Metabolism, Immune Response and Gut Systems. Front Microbiol. 2020;11(219). doi: 10.3389/fmicb.2020.00219.

59. Depommier C, Everard A, Druart C, Plovier H, Van Hul M, Vieira-Silva S, et al. Supplementation with Akkermansia muciniphila in overweight and obese human volunteers: a proof-of-concept exploratory study. Nature Medicine. 2019;25(7):1096-103. doi: 10.1038/s41591-019-0495-2.

60. Eslamparast T, Zamani F, Hekmatdoost A, Sharafkhah M, Eghtesad S, Malekzadeh R, et al. Effects of synbiotic supplementation on insulin resistance in subjects with the metabolic syndrome: a randomised, double-blind, placebocontrolled pilot study. British Journal of Nutrition. 2014;112(3):438-45. Epub 2014/05/22. doi: 10.1017/S0007114514000919.

61. Hernandez MAG, Canfora EE, Jocken JWE, Blaak EE. The Short-Chain Fatty Acid Acetate in Body Weight Control and Insulin Sensitivity. Nutrients. 2019;11(8). Epub 2019/08/21. doi: 10.3390/nu11081943. PubMed PMID: 31426593; PubMed Central PMCID: PMCPMC6723943.

62. van der Beek CM, Canfora EE, Lenaerts K, Troost FJ, Damink SW, Holst JJ, et al. Distal, not proximal, colonic acetate infusions promote fat oxidation and improve metabolic markers in overweight/obese men. Clin Sci (Lond). 2016;130(22):2073-82. Epub 2016/07/22. doi: 10.1042/cs20160263. PubMed PMID: 27439969.

63. Leighton F, Bergseth S, Rørtveit T, Christiansen EN, Bremer J. Free acetate production by rat hepatocytes during peroxisomal fatty acid and dicarboxylic acid oxidation. The Journal of biological chemistry. 1989;264(18):10347-50. PubMed PMID: 2732225.

64. van der Kolk BW, Vogelzangs N, Jocken JWE, Valsesia A, Hankemeier T, Astrup A, et al. Plasma lipid profiling of tissue-specific insulin resistance in human obesity. International journal of obesity (2005). 2019;43(5):989-98. Epub 2018/09/23. doi: 10.1038/s41366-018-0189-8. PubMed PMID: 30242234.

65. Stroeve JHM, Saccenti E, Bouwman J, Dane A, Strassburg K, Vervoort J, et al. Weight loss predictability by plasma metabolic signatures in adults with obesity and morbid obesity of the DiOGenes study. Obesity. 2016;24(2):379-88. doi: 10.1002/oby.21361.

66. Müller M, Hernández MAG, Goossens GH, Reijnders D, Holst JJ, Jocken JWE, et al. Circulating but not faecal short-chain fatty acids are related to insulin sensitivity, lipolysis and GLP-1 concentrations in humans. Scientific Reports. 2019;9(1):12515. doi: 10.1038/s41598-019-48775-0.

67. Fernandes J, Su W, Rahat-Rozenbloom S, Wolever TMS, Comelli EM. Adiposity, gut microbiota and faecal short chain fatty acids are linked in adult 
humans. Nutrition \& diabetes. 2014;4(6):e121-e. doi: 10.1038/nutd.2014.23. PubMed PMID: 24979150.

68. Zhao L. The gut microbiota and obesity: from correlation to causality. Nat Rev Microbiol. 2013;11(9):639-47. Epub 2013/08/06. doi: 10.1038/nrmicro3089. PubMed PMID: 23912213.

69. Müller M, Canfora EE, Blaak EE. Gastrointestinal Transit Time, Glucose Homeostasis and Metabolic Health: Modulation by Dietary Fibers. Nutrients. 2018;10(3):275. doi: 10.3390/nu10030275. PubMed PMID: 29495569.

70. Al-Lahham S, Roelofsen H, Rezaee F, Weening D, Hoek A, Vonk R, et al. Propionic acid affects immune status and metabolism in adipose tissue from overweight subjects. Eur J Clin Invest. 2012;42(4):357-64. Epub 2011/09/15. doi: 10.1111/j.1365-2362.2011.02590.x. PubMed PMID: 21913915.

71. Rumberger JM, Arch JR, Green A. Butyrate and other short-chain fatty acids increase the rate of lipolysis in 3T3-L1 adipocytes. PeerJ. 2014;2:e611. Epub 2014/10/17. doi: 10.7717/peerj.611. PubMed PMID: 25320679; PubMed Central PMCID: PMCPMC4193401.

72. Khan S, Jena G. Sodium butyrate reduces insulin-resistance, fat accumulation and dyslipidemia in type-2 diabetic rat: A comparative study with metformin. Chem Biol Interact. 2016;254:124-34. Epub 2016/06/09. doi: 10.1016/j.cbi.2016.06.007. PubMed PMID: 27270450.

73. Li Q, Chang Y, Zhang K, Chen H, Tao S, Zhang Z. Implication of the gut microbiome composition of type 2 diabetic patients from northern China. Scientific Reports. 2020;10(1):5450. doi: 10.1038/s41598-020-62224-3.

74. Qin J, Li Y, Cai Z, Li S, Zhu J, Zhang F, et al. A metagenome-wide association study of gut microbiota in type 2 diabetes. Nature. 2012;490(7418):5560. doi: 10.1038/nature11450.

75. Karlsson FH, Tremaroli V, Nookaew I, Bergström G, Behre CJ, Fagerberg B, et al. Gut metagenome in European women with normal, impaired and diabetic glucose control. Nature. 2013;498(7452):99-103. doi: 10.1038/nature12198.

76. Kaji I, Karaki S, Kuwahara A. Short-chain fatty acid receptor and its contribution to glucagon-like peptide-1 release. Digestion. 2014;89(1):31-6. Epub 2014/01/25. doi: 10.1159/000356211. PubMed PMID: 24458110.

77. Larraufie P, Martin-Gallausiaux C, Lapaque N, Dore J, Gribble FM, Reimann F, et al. SCFAs strongly stimulate PYY production in human enteroendocrine cells. Scientific Reports. 2018;8(1):74. doi: 10.1038/s41598-01718259-0.

78. Lee YY, Erdogan A, Rao SSC. How to assess regional and whole gut transit time with wireless motility capsule. J Neurogastroenterol Motil. 2014;20(2):265-70. doi: 10.5056/jnm.2014.20.2.265. PubMed PMID: 24840380.

79. Neyrinck AM, Van Hée VF, Piront N, De Backer F, Toussaint O, Cani PD, et al. Wheat-derived arabinoxylan oligosaccharides with prebiotic effect increase satietogenic gut peptides and reduce metabolic endotoxemia in diet-induced 
obese mice. Nutrition \& diabetes. 2012;2(1):e28-e. doi: 10.1038/nutd.2011.24. PubMed PMID: 23154683.

80. Christiansen CB, Gabe MBN, Svendsen B, Dragsted LO, Rosenkilde MM, Holst JJ. The impact of short-chain fatty acids on GLP-1 and PYY secretion from the isolated perfused rat colon. American Journal of Physiology-Gastrointestinal and Liver Physiology. 2018;315(1):G53-G65. doi: 10.1152/ajpgi.00346.2017. PubMed PMID: 29494208.

81. Gromada J, Chabosseau P, Rutter GA. The $\alpha$-cell in diabetes mellitus. Nature Reviews Endocrinology. 2018;14(12):694-704. doi: 10.1038/s41574-0180097-y.

82. Vogelzangs N, van der Kallen CJH, van Greevenbroek MMJ, van der Kolk BW, Jocken JWE, Goossens GH, et al. Metabolic profiling of tissue-specific insulin resistance in human obesity: results from the Diogenes study and the Maastricht Study. International Journal of Obesity. 2020. doi: 10.1038/s41366-020-0565-z.

83. Shimizu H, Masujima Y, Ushiroda C, Mizushima R, Taira S, Ohue-Kitano $\mathrm{R}$, et al. Dietary short-chain fatty acid intake improves the hepatic metabolic condition via FFAR3. Scientific Reports. 2019;9(1):16574. doi: 10.1038/s41598-01953242-x.

84. den Besten G, Bleeker A, Gerding A, van Eunen K, Havinga R, van Dijk TH, et al. Short-Chain Fatty Acids Protect Against High-Fat Diet-Induced Obesity via a PPAR $\gamma$-Dependent Switch From Lipogenesis to Fat Oxidation. Diabetes. 2015;64(7):2398. doi: 10.2337/db14-1213.

85. Lu Y, Fan C, Li P, Lu Y, Chang X, Qi K. Short Chain Fatty Acids Prevent High-fat-diet-induced Obesity in Mice by Regulating G Protein-coupled Receptors and Gut Microbiota. Scientific Reports. 2016;6(1):37589. doi: 10.1038/srep37589.

86. Li X, Chen H, Guan Y, Li X, Lei L, Liu J, et al. Acetic Acid Activates the AMP-Activated Protein Kinase Signaling Pathway to Regulate Lipid Metabolism in Bovine Hepatocytes. PLoS One. 2013;8(7):e67880. doi: 10.1371/journal.pone.0067880.

87. Frost G, Sleeth ML, Sahuri-Arisoylu M, Lizarbe B, Cerdan S, Brody L, et al. The short-chain fatty acid acetate reduces appetite via a central homeostatic mechanism. Nat Commun. 2014;5:3611. Epub 2014/05/02. doi: 10.1038/ncomms4611. PubMed PMID: 24781306; PubMed Central PMCID: PMCPMC4015327.

88. Kondo T, Kishi M, Fushimi T, Kaga T. Acetic Acid Upregulates the Expression of Genes for Fatty Acid Oxidation Enzymes in Liver To Suppress Body Fat Accumulation. Journal of Agricultural and Food Chemistry. 2009;57(13):59826. doi: 10.1021/jf900470c.

89. Chambers ES, Byrne CS, Rugyendo A, Morrison DJ, Preston T, Tedford C, et al. The effects of dietary supplementation with inulin and inulin-propionate ester on hepatic steatosis in adults with non-alcoholic fatty liver disease. Diabetes, Obesity and Metabolism. 2019;21(2):372-6. doi: 10.1111/dom.13500. 
90. Hage RE, Hernandez-Sanabria E, Arroyo MC, Wiele TVD. Supplementation of a propionate-producing consortium improves markers of insulin resistance in an in vitro model of gut-liver axis. American Journal of Physiology-Endocrinology and Metabolism. 0(0):null. doi: 10.1152/ajpendo.00523.2019. PubMed PMID: 31935110.

91. Frampton J, Murphy K, Frost G, Chambers E. Short-chain fatty acids as potential regulators of skeletal muscle metabolism and function. Nature Metabolism. 2020. doi: 10.1038/s42255-020-0188-7.

92. Inoue D, Tsujimoto G, Kimura I. Regulation of Energy Homeostasis by GPR41. Front Endocrinol (Lausanne). 2014;5(81). doi: 10.3389/fendo.2014.00081.

93. Ferchaud-Roucher V, Pouteau E, Piloquet H, Zaïr Y, Krempf M. Colonic fermentation from lactulose inhibits lipolysis in overweight subjects. Am J Physiol Endocrinol Metab. 2005;289(4):E716-20. Epub 2005/09/10. doi: 10.1152/ajpendo.00430.2004. PubMed PMID: 16150956.

94. Pouteau E, Vahedi K, Messing B, Flourié B, Nguyen P, Darmaun D, et al. Production rate of acetate during colonic fermentation of lactulose: a stableisotope study in humans. Am J Clin Nutr. 1998;68(6):1276-83. Epub 1998/12/10. doi: 10.1093/ajcn/68.6.1276. PubMed PMID: 9846859.

95. Luo J, Rizkalla SW, Alamowitch C, Boussairi A, Blayo A, Barry JL, et al. Chronic consumption of short-chain fructooligosaccharides by healthy subjects decreased basal hepatic glucose production but had no effect on insulinstimulated glucose metabolism. Am J Clin Nutr. 1996;63(6):939-45. Epub 1996/06/01. doi: 10.1093/ajcn/63.6.939. PubMed PMID: 8644690.

96. Boll EV, Ekstrom LM, Courtin CM, Delcour JA, Nilsson AC, Bjorck IM, et al. Effects of wheat bran extract rich in arabinoxylan oligosaccharides and resistant starch on overnight glucose tolerance and markers of gut fermentation in healthy young adults. Eur J Nutr. 2016;55(4):1661-70. Epub 2015/07/15. doi: 10.1007/s00394015-0985-z. PubMed PMID: 26169871.

97. Sakoda H, Ogihara T, Anai M, Fujishiro M, Ono H, Onishi Y, et al. Activation of AMPK is essential for AICAR-induced glucose uptake by skeletal muscle but not adipocytes. Am J Physiol Endocrinol Metab. 2002;282(6):E1239-44. Epub 2002/05/15. doi: 10.1152/ajpendo.00455.2001. PubMed PMID: 12006353.

98. Musi N, Goodyear LJ. AMP-activated protein kinase and muscle glucose uptake. Acta physiologica Scandinavica. 2003;178(4):337-45. Epub 2003/07/17. doi: 10.1046/j.1365-201X.2003.01168.x. PubMed PMID: 12864738.

99. Kjøbsted R, Hingst JR, Fentz J, Foretz M, Sanz M-N, Pehmøller C, et al. AMPK in skeletal muscle function and metabolism. FASEB journal : official publication of the Federation of American Societies for Experimental Biology. 2018;32(4):1741-77. Epub 2018/01/05. doi: 10.1096/fj.201700442R. PubMed PMID: 29242278.

100. Tobias IS, Lazauskas KK, Siu J, Costa PB, Coburn JW, Galpin AJ. Sex and fiber type independently influence AMPK, TBC1D1, and TBC1D4 at rest and during recovery from high-intensity exercise in humans. Journal of applied 
physiology (Bethesda, Md : 1985). 2020;128(2):350-61. Epub 2020/01/03. doi: 10.1152/japplphysiol.00704.2019. PubMed PMID: 31895596.

101. Pannacciulli N, Bunt JC, Koska J, Bogardus C, Krakoff J. Higher fasting plasma concentrations of glucagon-like peptide 1 are associated with higher resting energy expenditure and fat oxidation rates in humans. Am J Clin Nutr. 2006;84(3):556-60. Epub 2006/09/09. doi: 10.1093/ajcn/84.3.556. PubMed PMID: 16960169.

102. Karra E, Chandarana K, Batterham RL. The role of peptide YY in appetite regulation and obesity. J Physiol. 2009;587(1):19-25. Epub 2008/12/08. doi: 10.1113/jphysiol.2008.164269. PubMed PMID: 19064614.

103. Bajpeyi S, Myrland CK, Covington JD, Obanda D, Cefalu WT, Smith SR, et al. Lipid in skeletal muscle myotubes is associated to the donors' insulin sensitivity and physical activity phenotypes. Obesity (Silver Spring, Md). 2014;22(2):426-34. Epub 2013/09/10. doi: 10.1002/oby.20556. PubMed PMID: 23818429.

104. Ukropcova B, McNeil M, Sereda O, de Jonge L, Xie H, Bray GA, et al. Dynamic changes in fat oxidation in human primary myocytes mirror metabolic characteristics of the donor. J Clin Invest. 2005;115(7):1934-41. doi: 10.1172/JCI24332. PubMed PMID: 16007256.

105. Blachier F, Beaumont M, Portune KJ, Steuer N, Lan A, Audebert M, et al. High-protein diets for weight management: Interactions with the intestinal microbiota and consequences for gut health. A position paper by the my new gut study group. Clinical Nutrition. 2019;38(3):1012-22. doi: 10.1016/j.clnu.2018.09.016. 106. Tiihonen K, Ouwehand AC, Rautonen N. Effect of overweight on gastrointestinal microbiology and immunology: correlation with blood biomarkers. British Journal of Nutrition. 2010;103(7):1070-8. Epub 2009/11/24. doi: 10.1017/S0007114509992807.

107. Aragonès G, Colom-Pellicer M, Aguilar C, Guiu-Jurado E, Martínez S, Sabench F, et al. Circulating microbiota-derived metabolites: a "liquid biopsy? International Journal of Obesity. 2020;44(4):875-85. doi: 10.1038/s41366-019-0430-0. 108. Feng W, Ao H, Peng C. Gut Microbiota, Short-Chain Fatty Acids, and Herbal Medicines. Frontiers in Pharmacology. 2018;9(1354). doi: 10.3389/fphar.2018.01354.

109. den Besten G, van Eunen K, Groen AK, Venema K, Reijngoud D-J, Bakker $\mathrm{BM}$. The role of short-chain fatty acids in the interplay between diet, gut microbiota, and host energy metabolism. J Lipid Res. 2013;54(9):2325-40. Epub 2013/07/02. doi: 10.1194/jlr.R036012. PubMed PMID: 23821742.

110. Tang Z-Z, Chen G, Hong Q, Huang S, Smith HM, Shah RD, et al. MultiOmic Analysis of the Microbiome and Metabolome in Healthy Subjects Reveals Microbiome-Dependent Relationships Between Diet and Metabolites. Frontiers in Genetics. 2019;10(454). doi: 10.3389/fgene.2019.00454.

111. Nagpal R, Mainali R, Ahmadi S, Wang S, Singh R, Kavanagh K, et al. Gut microbiome and aging: Physiological and mechanistic insights. Nutr Healthy Aging. 2018;4(4):267-85. doi: 10.3233/NHA-170030. PubMed PMID: 29951588. 
112. Zhong H, Penders J, Shi Z, Ren H, Cai K, Fang C, et al. Impact of early events and lifestyle on the gut microbiota and metabolic phenotypes in young school-age children. Microbiome. 2019;7(1):2. doi: 10.1186/s40168-018-0608-z.

113. Fernandes J, Vogt J, Wolever TMS. Inulin increases short-term markers for colonic fermentation similarly in healthy and hyperinsulinaemic humans. European Journal Of Clinical Nutrition. 2011;65:1279. doi: 10.1038/ejcn.2011.116.

114. Meslier V, Laiola M, Roager HM, De Filippis F, Roume H, Quinquis B, et al. Mediterranean diet intervention in overweight and obese subjects lowers plasma cholesterol and causes changes in the gut microbiome and metabolome independently of energy intake. Gut. 2020:gutjnl-2019-320438. doi: 10.1136/gutjnl2019-320438.

115. Rodriguez J, Hiel S, Neyrinck AM, Le Roy T, Pötgens SA, Leyrolle Q, et al. Discovery of the gut microbial signature driving the efficacy of prebiotic intervention in obese patients. Gut. 2020:gutjnl-2019-319726. doi: 10.1136/gutjnl2019-319726.

116. Trouwborst I, Bowser SM, Goossens GH, Blaak EE. Ectopic Fat Accumulation in Distinct Insulin Resistant Phenotypes; Targets for Personalized Nutritional Interventions. Front Nutr. 2018;5:77-. doi: 10.3389/fnut.2018.00077. PubMed PMID: 30234122.

117. Most J, Goossens GH, Reijnders D, Canfora EE, Penders J, Blaak EE. Gut microbiota composition strongly correlates to peripheral insulin sensitivity in obese men but not in women. Benef Microbes. 2017;8(4):557-62. Epub 2017/06/18. doi: 10.3920/bm2016.0189. PubMed PMID: 28618864. 
APPENDIX 


\section{Summary}

In the last two decades, evidence for a link between the gut microbiome with the host metabolism and metabolic disorders like insulin resistance and type 2 diabetes mellitus has constantly increased. The gut microbiota can ferment indigestible carbohydrates (saccharolytic fermentation) and produce the major short chain fatty acids (SCFA) acetate, propionate and butyrate mainly in the proximal colon. Of relevance, SCFA have been linked to improvements in substrate handling at the tissue level contributing to an amelioration in insulin sensitivity and metabolic health. Additionally, fermentation of peptides and proteins (proteolytic fermentation), mainly occurring in the distal colon, yields products such as branched chain fatty acids (BCFA), which may have adverse effects on gut and metabolic health. However, most literature is based on animal studies and only a limited amount of human studies are available. Hence, in this thesis we focused on the role of gut-derived metabolites (SCFA and BCFA) in in vivo metabolic health in human cross-sectional and intervention studies with overweight and obese individuals as well as the role of SCFA in human adipose tissue and skeletal muscle derived in vitro models.

In chapter 2, we extensively reviewed findings on the most abundant SCFA acetate in the context of body weight control and insulin sensitivity. We discuss that circulating acetate may have tissue-specific metabolic effects (liver, skeletal muscle, adipose tissue and pancreatic beta cells) and potentially impact wholebody energy and substrate metabolism (satiety, energy expenditure, fat oxidation) resulting in an improved metabolic health and insulin sensitivity. In addition, we discuss that the effectiveness of interventions (pre- probiotics, synbiotics) aiming to increase acetate concentrations in the colon as well as in the circulation may depend on colonic production site. In this respect, differences in the site of acetate production (proximal vs distal colon) as well as the differences in colonic acetate release into the circulation have to be taken into account. Furthermore, we discuss that future interventions (using prebiotics, probiotics, synbiotics and/or vinegar administrations) with the aim to increase circulating acetate should be more personalized and thereby consider both metabolic and microbial phenotype of participants.

Interestingly, it has been shown that the relationship between gut microbiota and insulin sensitivity may be sex-specific in humans. Therefore, in chapter 3, we investigated whether the associations between circulating acetate with insulin sensitivity/resistance indices (Homeostatic Model Assessment of Insulin Resistance (HOMA-IR), circulating insulin and Matsuda index) were different in male and female participants (BMI $>27 \mathrm{~kg} / \mathrm{m}^{2}, \mathrm{n}=478$ ) of the Diet, Obesity and Genes (DiOGenes) study before and after a low-calorie diet (LCD, 800 $\mathrm{kcal} / \mathrm{d})$. We found that acetate was positively associated with insulin resistance in females, but not in males, even after adjustment for age, BMI and fat free mass. 
Of note, these findings warrant further investigations to understand the sex-specific differences in circulating acetate and their potential role in metabolic health during weight loss. In particular, factors contributing to circulating fasting acetate such as hormonal status, endogenous liver production (ketogenesis) and/or the gut-derived production of acetate should be considered. Furthermore, future studies should investigate acetate dynamics to fully elucidate the role of circulating acetate in metabolic health.

So far, in human studies gut microbial-derived SCFA have been mainly measured in feces. However, fecal SCFA production should be interpreted with caution, since they are the net result of colonic production, absorption rates and release into the circulation, which are intricately influenced by microbial composition, cross-feeding mechanisms and whole gut transit time among other factors. Therefore, in chapter 4, we investigated associations between fecal and plasma SCFA (acetate, propionate and butyrate) with metabolic health markers including circulating metabolites, gut hormones, substrate oxidation, inflammatory parameters and markers of insulin sensitivity in a well-phenotyped group of individuals with a large range in BMI and glucometabolic status. Interestingly, we found that plasma, not fecal, SCFA (acetate, propionate and butyrate) associated negatively with circulating markers of lipid metabolism and positively with circulating GLP-1 levels. The negative associations with circulating lipids (glycerol, triglycerides, free fatty acids, respectively) are in line with previous literature, suggesting a relationship between SCFA and adipose tissue metabolism and possible improvements in adipose tissue lipid buffering capacity. An interesting (novel) finding was the positive association of circulating SCFA with fasting GLP-1 levels possibly indicating a link of plasma SCFA with guthormone secretion. In a subgroup analysis, we found that insulin sensitivity (as measured by the golden standard hyperinsulinaemic-euglycemic clamp technique) associated with circulating SCFA but that the direction of this association depended on type of SCFA (acetate negatively and propionate positively). Although controversial associations of circulating acetate and insulin sensitivity have been found (in chapter 3 and chapter 4), findings should be interpreted with caution taking into consideration that circulating acetate is affected by various factors including liver endogenous production (ketogenesis), gut-derived production as well as metabolic and microbial phenotype.

Besides saccharolytic fermentation and SCFA production, proteolytic fermentation may yield various metabolites such as BCFA (i.e. isobutyrate, isovalerate), which role in human substrate and energy metabolism remains largely unclear. In addition, research on other fecal metabolites derived from saccharolytic fermentation (i.e. valerate, lactate) is largely lacking. Therefore, in chapter 5, we investigated fecal BCFA (isobutyrate and isovalerate) and other fecal microbial metabolites (valerate, lactate, succinate and caproate) in insulin sensitive and insulin resistant individuals. Additionally, we assessed the relationship of fecal microbial metabolites with circulating metabolites, substrate oxidation, and 
markers of insulin sensitivity (circulating insulin and HOMA-IR). Here, we found no significant differences in fecal BCFA concentrations between insulin sensitive and insulin resistant individuals. Additionally, there were no associations between BCFA and markers of insulin resistance and metabolic health. Further human studies are warranted to investigate the role of these microbially-derived fermentation products and their kinetics in metabolic health and insulin sensitivity.

In chapter 6, we investigated whether our previously observed increments in in vivo fat oxidation after colonic acetate administration in humans were mediated through direct effects of circulating acetate on the skeletal muscle fat oxidation. Therefore, we investigated the effects of sodium acetate on fat oxidation in human primary muscle cells (HSkMC) derived from a healthy insulin sensitive donor. We observed no direct sodium acetate-mediated effects neither on endogenous nor on exogenous fat oxidation in our human skeletal muscle cell model using physiologically relevant sodium acetate concentrations. Additionally, we observed no dose and time effect of sodium acetate on total and phosphorylated AMPK levels. However, we cannot exclude that our previously observed in vivo human effects on fat oxidation after colonic acetate administrations occur in tissues other than skeletal muscle (i.e. liver fat oxidation) or are mediated by other gut-derived metabolites or hormones (i.e. PYY). Additionally, the lack of effect on in vitro muscle fat oxidation may be donordependent, muscle fiber type-specific as well as species-specific and warrants further investigation.

In several animal as well as human in vivo studies an anti-lipolytic effect of SCFA was demonstrated, which may reduce systemic lipid overflow and ultimately result in improvements in insulin sensitivity. Therefore, in chapter 7 , we used the human multipotent adipose-derived stem (hMADS) cell model to study the effects of SCFA on (cytosolic) lipolysis and to elucidate the potential underlying mechanisms. We found that SCFA differentially affect adipose tissue lipolysis, and in particular that acetate is the major SCFA responsible for the decreased basal and $\beta$-adrenergically stimulated glycerol release in our in vitro hMADS cell model. The latter anti-lipolytic effect was mediated through a GPRdependent reduction in phosphorylation of hormone sensitive lipase (HSL). Thus, these data suggest that circulating acetate affect adipose tissue lipolysis, thereby possibly limiting lipid overflow and improving insulin sensitivity.

In conclusion, this doctoral thesis provides an increased insight in the role of saccharolytic (major SCFA as well as other less studied metabolites) and proteolytic fermentation products (BCFA) in insulin sensitivity and substrate metabolism in human cohorts as well as in in vitro studies. The main findings indicate that gut-derived metabolites (SCFA) in the circulation might be a better indicator of their effects on metabolic health as compared to fecal concentrations.

Furthermore, the present data suggest that the sex-specific relationship of circulating acetate with insulin sensitivity exist, which may depend on various 
factors such as endogenous liver metabolism, hormonal profile, and microbial and metabolic phenotype.

Lastly, our in vitro experiments provided valuable mechanistic insight on the relation between acetate and skeletal muscle fat oxidation and adipose tissue lipolysis, which needs to be investigated in more detail in human in vitro and in vivo studies. Additionally, future studies need to consider SCFA and BCFA dynamics (production, absorption and release into the circulation), colonic production site as well as the role of these gut-derived metabolites in tissue and whole-body metabolism and metabolic health. 


\section{Resumen}

En las ultimas décadas, ha aumentado la evidencia de la conexión de la microbiota intestinal con el metabolismo del huésped, así como con enfermedades como la Resistencia a la insulina y la diabetes tipo 2. La microbiota intestinal posee la capacidad de fermentar hidratos de carbono no digeribles (i.e. fibra, prebióticos) y producir ácidos grasos de cadena corta (SCFA, por sus siglas en inglés) principalmente en la parte proximal del intestino grueso. Los SCFA de mayor relevancia son acetato, propionato y butirato. La literatura ha reportado que los SCFA están relacionados con mejoras del metabolismo en distintos tejidos, así como mejoras en la sensibilidad a la insulina. En la parte distal del intestino grueso, el microbiota intestinal fermenta péptidos y proteínas y produce ácidos grasos de cadena ramificada (BCFA por sus siglas en ingles) entre otros metabolitos, los cuales pueden tener efectos adversos en el intestino y en la salud metabólica. La mayor parte de la evidencia al respecto de estos metabolitos proviene de estudios en animales. Por lo tanto, en esta tesis, nos enfocamos en el rol de SCFA y BCFA en estudios in vivo en humanos con sobrepeso y obesidad, así como el rol de los SCFA en modelos in vitro de tejido adiposo y musculo esquelético derivados de humanos.

En el capitulo 2, realizamos una revisión bibliográfica de la literatura del SCFA acetato en contexto de control de peso y mejora de la sensibilidad a la insulina. En el cual, discutimos que las concentraciones de acetato en la sangre pueden tener efectos en distintos órganos importantes (hígado, musculo esquelético, tejido adiposo y las células pancreáticas beta), impactar en el metabolismo sistémico de macronutrimentos (saciedad, gasto energético, oxidación de grasas) y a su vez mejorar la salud metabólica y disminuir la resistencia a la insulina. Además, discutimos que la efectividad de las intervenciones (pre, probióticos y sinbióticos) con el objetivo de incrementar las concentraciones de acetato en el intestino grueso y en la circulación sistémica dependen del sitio de producción en el intestino grueso. Respecto a esto, diferencias de producción de acetato en el intestino grueso (proximal versus distal) así como diferencias en la absorción a la circulación deben ser consideradas. Por ultimo, en la revisión bibliográfica discutimos que las futuras intervenciones (pre, probióticos y sinbióticos y/o vinagre) con el objetivo de incrementar concentraciones de acetato en sangre deben ser personalizadas y considerar la influencia del fenotipo metabólico y de la microbiota intestinal en los individuos. 
Curiosamente, se ha reportado una relación entre la microbiota intestinal y la sensibilidad a la insulina con diferencias entre sexos en humanos. Por lo tanto, en el capitulo 3 investigamos si la relación entre las concentraciones de acetato con la sensibilidad a la insulina (Homeostatic Model Assessment of Insulin Resistance (HOMA-IR), insulina en sangre y Matsuda Index) eran distintas entre hombres y mujeres (IMC $>27 \mathrm{~kg} / \mathrm{m}^{2}, \mathrm{n}=478$ ), participantes del estudio DiOGenes (Diet, Obesity and Genes) antes y después de una dieta baja en calorías ( $800 \mathrm{kcal} / \mathrm{d}$ ). Encontramos que el acetato estaba positivamente asociado con resistencia a la insulina en mujeres y no en hombres, incluso después de ajustar por edad, IMC y masa magra. Estos hallazgos deben ser investigados a fondo para entender las diferencias en acetato en sangre entre sexos, así como el rol del acetato en la salud metabólica durante la perdida de peso. En particular, se deben investigar los factores que contribuyen a las concentraciones de acetato en ayunas tales como el estatus hormonal, cetogénesis endógena del hígado y/o la producción de acetato en el intestino grueso. Además, estudios futuros deben investigar el flujo de acetato del intestino grueso a la circulación sistémica y su rol en la salud metabólica.

Actualmente, estudios en humanos que estudian los SCFA derivados de la fermentación en el intestino grueso han medido las concentraciones en heces. Sin embargo, las concentraciones de SCFA en heces deben ser interpretadas con cautela, ya que son el resultado de la producción en el intestino grueso, velocidad de absorción y liberación a la circulación sistémica, procesos que están intricadamente controlados por la composición de la microbiota intestinal, mecanismos de alimentación cruzada (cross-feeding), transito intestinal entre otros factores. Por lo tanto, en el capitulo 4, investigamos las asociaciones entre concentraciones de SCFA (acetato, propionato y butirato) en heces y sangre con marcadores metabólicos incluyendo metabolitos en sangre, hormonas intestinales, calorimetría indirecta, marcadores de inflamación y marcadores de sensibilidad a la insulina en un grupo de individuos con amplio rango de IMC y de estatus glucometabolico. Curiosamente, encontramos que SCFA (acetato, propionato y butirato) en sangre, no en heces, están asociados negativamente con marcadores de metabolismo de lípidos y positivamente asociados con niveles de péptido similar al glucagón 1 (GLP-1) en sangre. Las asociaciones negativas con lípidos en sangre (glicerol, triglicéridos, ácidos grasos libres, respectivamente) coinciden con la literatura, lo que sugiere una relación entre SCFA con metabolismo del tejido adiposo y posibles beneficios en la capacidad de almacenamiento del tejido adiposo. Un hallazgo interesante es la asociación positiva de los SCFA en sangre con niveles de GLP-1 en sangre, posiblemente indicando una conexión de SCFA en sangre con secreción de GLP-1. En el capitulo 4, en un análisis de un subgrupo encontramos que la sensibilidad a la insulina (medida con la técnica hiperinsulinémico-euglucémico clamp) se asocio con SCFA en sangre en direcciones opuestas (acetato negativamente y propionato positivamente). 
Aunque las asociaciones de niveles en sangre de acetato con insulina son controversiales (capitulo 3 y capitulo 4), estas deben ser interpretadas con cautela considerando que los niveles de acetato en sangre son afectados por varios factores incluyendo la producción endógena por medio de la cetogénesis en el hígado, producción del intestino grueso, así como el fenotipo metabólico y de la microbiota intestinal.

Aparte de la fermentación de los hidratos de carbono no digeribles, la fermentación de proteínas y péptidos en el intestino grueso distal puede producir varios metabolitos tales como los BCFA (isobutirato e isovalerato), cuyo rol en la salud metabólica y metabolismo sistémico de macronutrimentos permanece sin investigar. Además, que existe carente investigación respecto a otros metabolitos presentes en heces derivados de la fermentación de hidratos de carbono no digeribles (i.e. valerato, lactato). Por lo tanto, en el capitulo 5, investigamos las concentraciones de metabolitos en heces incluyendo BCFA (isobutirato, isovalerato), otros metabolitos (valerato, lactato, succinato y caproato) en individuos con sensibilidad y resistencia a la insulina. Además, evaluamos la relación entre las concentraciones de estos metabolitos en heces con metabolitos en sangre, calorimetría indirecta y marcadores de sensibilidad a la insulina (insulina en sangre y HOMA-IR). No encontramos diferencias significativas en BCFA entre individuos con sensibilidad e individuos con resistencia a la insulina. Además, no encontramos asociaciones entre BCFA y marcadores de resistencia a la insulin y salud metabólica. Estudios futuros deben investigar el rol de estos metabolitos, así como su flujo del intestino grueso hacia la circulación sistémica.

En el capitulo 6, investigamos si los resultados previos en humanos ocurren a nivel del musculo esquelético, en los que la administración de acetato en el intestino grueso causaba incrementos en la oxidación de grasas. Por lo tanto, investigamos los efectos del acetato de sodio en la oxidación de grasas en células de musculo esquelético derivadas de una biopsia de un individuo sano (HSkMC). No observamos un efecto directo del acetato de sodio en oxidación de grasas (endogena y exógena) en nuestro modelo HSkMC usando concentraciones fisiológicas de acetato de sodio. Además, no observamos un efecto de dosis o de tiempo en la fosforilación de AMPK. Sin embargo, no podemos excluir que nuestros efectos reportados en humanos en la oxidación de grasas después de una administración en el intestino grueso ocurren en otros tejidos (hígado) o son mediados por otros metabolitos (butirato) u hormonas (PYY). Además, nuestra falta de efecto en la oxidación de grasas en respuesta a acetato de sodio puede ser explicada por diferencias del donante, tipo de fibra muscular as como por diferencias entre especies. 
Varios estudios en animales, así como estudios en humanos han reportado un efecto anti-lipolítico de los SCFA, el cual puede reducir los niveles sistémicos de dislipidemia y mejorar la sensibilidad a la insulina. Por lo tanto, en el capitulo 7, usamos el modelo celular de adipocito derivado de humanos (human multipotent adipose-derived stem (hMADS) para estudiar los efectos de SCFA en la lipolisis citosolica y elucidar los mecanismos. Encontramos que los SCFA modulan la lipolisis de diferente forma, y en particular el acetato es el mayor responsable de disminuir la liberación basal y estimulada ( $\beta$-adrenérgica) de glicerol en nuestro modelo hMADS. Este efecto anti-lipolítico fue mediado por la reducción de la fosforilación de la hormona sensitiva a la lipasa (HSL) por medio de los receptores celulares GPR. Estos resultados sugieren que el acetato en sangre disminuye la lipolisis y muestra potencial para mejorar la dislipidemia y resistencia a la insulina.

En conclusión, esta tesis doctoral provee mayor información respecto al rol metabólico de los metabolitos que provienen de la fermentación de hidratos de carbono no digeribles (principales SCFA y otros menos abundantes) y de los metabolitos provenientes de la fermentación de proteínas y péptidos (BCFA) en la sensibilidad a la insulina y metabolismo de macronutrimentos en estudios de cohorte en humanos así como en estudios con modelos celulares derivados de humanos (musculo esquelético y tejido adiposo). Los principales hallazgos indican que las concentraciones de SCFA en sangre pueden ser mejores indicadores de los efectos en la salud metabólica, en comparación con niveles de SCFA medidos en heces. Además, estos resultados sugieren que la relación de los niveles de acetato en sangre con la sensibilidad a la insulina puede ser distinta en hombres y en mujeres y depender de varios factores como metabolismo del hígado, perfil hormonal, y fenotipo metabólico y de microbiota intestinal. Por ultimo, nuestros experimentos in vitro proveen más información a nivel de mecanismos respecto a metabolismo de SCFA en oxidación de grasas en musculo esquelético y de efectos en lipolisis en tejido adiposo, que debe ser investigado en mas detalle en estudios futuros. Además, estudios futuros deben considerar el sitio de producción, absorción, y liberación en el intestino grueso de los SCFA y BCFA, su contribución al metabolismo de distintos órganos y efectos sistémicos en la salud metabólica. 


\section{Valorization}

This thesis describes the potential of gut-derived microbial metabolites as key players in host metabolic health and insulin sensitivity. We investigated the role of short chain fatty acids and branched chain fatty acids (SCFA and BCFA) in in vivo metabolic health in human cross-sectional and intervention studies with overweight people and with obesity. In addition, we studied the role of SCFA in human derived adipose and skeletal muscle tissues in in vitro models. In this section, we describe the impact of our scientific findings for global society and economy, especially on the implications and possible applications for specific target groups with higher health risks.

Obesity is associated with other comorbidities including cardiovascular disease, type 2 diabetes mellitus, some forms of cancer among other comorbidities 1. Currently, obesity prevalence and related complications are continuing to increase as reported by the World Health Organization with prevalence rates of obesity of 2 billion according to 2016 report ${ }^{1}$. Most affected areas include parts of Europe, North America (i.e. Mexico) and the Middle East ${ }^{1}$. Of major importance, predictions point towards even higher increments in the near future.

Obesity is a chronic metabolic disease that results from an energy imbalance in the long-term. The major contributing factors to complications include an increased lipid accumulation in adipose and non-adipose organs, adipose tissue dysfunction, lipid spillover and low-grade inflammation that collectively result in the development of insulin resistance. These metabolic alterations may start with a mild insulin resistant state and progress to type 2 diabetes mellitus.

In the last decades, research has shown the intricate connection of the gut and gut microbial-derived metabolites with host metabolic health. In this context, this thesis provides valuable information about major saccharolytic fermentation products (acetate, propionate and butyrate) and less abundant gut-derived metabolites (lactate, caproate, valerate, succinate) as well as proteolytic fermentation products (isovalerate and isobutyrate). This sheds light on the individual as well as collective effects of these metabolites on host metabolic health and insulin sensitivity.

For instance, we discuss the possible higher relevance of circulating SCFA as compared to fecal concentrations as biomarkers of metabolic health effects. Although this particular finding warrants further investigation, this may be of importance in the clinical setting and monitoring of nutritional interventions targeting the gut. Furthermore, we show that the relationship of the major SCFA acetate with insulin sensitivity together with the sexual dimorphism in this 
relationship is complex and warrants further investigation. Of note, these findings point towards the need for carefully designed studies to investigate SCFA (i.e. acetate) kinetics (production, absorption and release into the circulation) in relation to metabolic health.

In general, this thesis adds to the knowledge on the potential of gut-derived metabolites to modulate host metabolic health, especially in the human situation. In this regard, our findings may provide useful information for dietary intervention strategies that may ameliorate metabolic health. It is evident that attention should be placed on the balance between saccharolytic and proteolytic fermentation as a determinant of metabolic health. Furthermore, our data may provide clues for a more targeted dietary guidance based on microbial and metabolic phenotype. After further confirmation of these findings in prospective dietary intervention studies, this knowledge may be used by health care professionals when advising patients on their dietary intake as well as relevant leads for food industry to produce functional foods with pronounced metabolic benefits.

Of note, the in vitro studies of this doctoral thesis provided valuable scientific input on tissue-specific effects of SCFA. These studies provided important mechanistic insight on previous described metabolic effect on lipolysis and fat oxidation after colonic SCFA administration in vivo. However, more research is needed to define effective dietary interventions (pre, pro, synbiotics) that can increase systemic SCFA levels in physiologically relevant concentrations, thereby eliciting similar effects, as reported in vitro, at the tissue level in skeletal muscle and adipose tissue or other tissues.

This thesis was partly funded by a Kootstra Talent grant from Maastricht University Medical Centre ${ }^{+}$obtained by Emanuel Canfora. In addition, this thesis was possible through a Scholarship granted to Manuel Gonzalez by Consejo Nacional de Ciencia y Tecnologia (CONACYT), a public agency of Mexico's federal government. In this thesis, we used large dataset including data from the large European project DiOGenes. In this context, this thesis contributes to a broader and cross atlantic/global perspective that stimulated collaboration and benefited all parties in gaining knowledge concerning obesity.

All the work in this thesis will be available to the public through scientific publications in internationally peer-reviewed journals. In addition, there has been frequent interaction in research meetings, symposiums (School of Nutrition and Translational Research in Metabolism, NUTRIM) and conferences at the national (Netherlands Association for the Study of Obesity, NASO Utrecht 2018 and 2019) and international level (European Congress of Obesity, ECO Vienna 2018 and Glasgow 2019). 
The overall aim of the thesis was to gain more insight into gut microbial metabolites and their impact on metabolic health by utilizing state of the art methodology and extensive human phenotyping. In addition, we used human derived skeletal muscle and adipose tissue in vitro models, which provided valuable insight into human physiology that was previously lacking.

Previous work has focused on identifying nutritional interventions (i.e. prebiotics) and their capacity to produce microbial metabolites that can improve metabolic health. Accumulating evidence suggests that the obesity pandemic may increase susceptibility and worsen the current coronavirus disease 2019 (COVID19) outcomes possibly partially linked to alterations in microbial community ${ }^{2,3}$. However, future research has to elucidate whether nutritional interventions promoting anti-inflammatory properties, gut and metabolic health may ameliorate COVID-19 infections and the progression of the disease ${ }^{4}$.

To conclude, this thesis provides input on the role of saccharolytic (SCFA) and proteolytic fermentation products (BCFA) in metabolic health in human studies and human derived cell models. Of note, this may lead to design more carefully and personalized human interventions and investigate in more detail the role of microbial metabolites in metabolic health in response to nutritional interventions. Finally, this may aid in the development of more personalized nutritional strategies that counteract the impact of obesity and its comorbidities more effectively than or in addition to conventional caloric restriction. 


\section{References}

1. Engin A. The Definition and Prevalence of Obesity and Metabolic Syndrome. Adv Exp Med Biol. 2017;960:1-17. Epub 2017/06/07. doi: 10.1007/978-3319-48382-5_1. PubMed PMID: 28585193.

2. Goossens GH, Dicker D, Farpour-Lambert NJ, Frühbeck G, Mullerova D, Woodward E, et al. Obesity and COVID-19: A Perspective from the European Association for the Study of Obesity on Immunological Perturbations, Therapeutic Challenges, and Opportunities in Obesity. Obesity Facts. 2020. doi: 10.1159/000510719.

3. Dhar D, Mohanty A. Gut microbiota and Covid-19- possible link and implications. Virus Research. 2020;285:198018. doi: 10.1016/j.virusres.2020.198018. 4. Iddir M, Brito A, Dingeo G, Fernandez Del Campo SS, Samouda H, La Frano MR, et al. Strengthening the Immune System and Reducing Inflammation and Oxidative Stress through Diet and Nutrition: Considerations during the COVID-19 Crisis. Nutrients. 2020;12(6):1562. doi: 10.3390/nu12061562. PubMed PMID: 32471251. 


\section{Acknowledgments}

I feel grateful for being part of the now called NIMO lab group. First, I would like to thank my promotor Ellen Blaak for her guidance and support during these years in the department of Human Biology that helped me develop the academic skills.

Second, I would like to express my gratitude to my co-promotor Emanuel Canfora for his support and quick responses to my questions and emails. Thanks for sharing your thoughts on the field and the recent articles.

I am also grateful for the guidance from my co-promotor Johan Jocken who always had multiple ways to plan experiments. Thanks for providing the support to gain confidence in developing the cell culture skills and perform the experiments on my own.

Thank you to Prof. dr. Marleen van Baak, Prof. dr. Koen Venema, Dr. Gerben Hermes, Dr. Freddy Troost and Prof. dr. Patrick Rensen for your time to evaluate and review my thesis as well as for being present at the official $\mathrm{PhD}$ defense.

My paranymphs from the "acetic group" when I started learning cell culture back from the beginning of the PhD, Jacco and Kenneth. Thank you for your time for planning and organize the defense details.

To Gijs and Ruth, thank you for your interesting contributions during research and literature meetings as well as warm conversations in the corridor.

Nicole and Yvonne, thank you for your guidance and help with all the technical preparations for the experiments and cell culture learning path. Thanks as well for your kind support along the way.

To all my former and current colleagues: Rudi, Kenneth Verboven, Max, Birgitta, Mattea, Kenneth, Jacco, Rens, Inez, Kelly, Qing, Lars, Michelle, Dilemin, Mirella, Ioannis, Jehad, Sultan, Lina and Thirza. Thanks for sharing the path

To all colleagues in Humane Biologie department, this has been a great life experience, I thank you for your kindness and I certainly will miss the Vlaai meetings. 
Gracias a mi familia en México por su apoyo y sus mensajes durante los años de mi estancia. Aunque por motivos de la pandemia no pudieron asistir al evento final, los tengo siempre presentes. A mi abuelita Ramoncita con cariño que esta siempre contenta y cocinando.

A mis padres Constancio y Verónica y mi hermano Jonatán, siempre los tengo presentes a pesar de la distancia. Gracias por su apoyo y sus visitas durante este periodo en Maastricht. Gracias a mis padres por su ejemplo y por enseñarnos una educación integral y fomentar en nosotros el deporte y la música que son grandiosos acompañantes al estar lejos de México. A mi tío Alejandro, gracias que fue ejemplo desde niño para perseguir el objetivo de un doctorado. 


\section{Curriculum Vitae}

Manuel Alejandro Gonzalez Hernandez was born on May 12 1989 in Leon Guanajuato, México, where he was raised and completed his bachelor's degree in Human Nutrition at Universidad de Guanajuato in 2013. During undergraduate studies he enrolled in an exchange program for one semester in St. Francis Xavier University (Nova Scotia, Canada) and joined a summer research program at University of Arizona, (Tucson, USA). He continued his education in the Netherlands, where he obtained his master's degree in Biomedical Sciences at Maastricht University in 2016 at the department of Human Biology.

In 2016, Manuel started his PhD at the department of Human Biology at Maastricht University within NUTRIM School of Nutrition and Translational Research in Metabolism under the supervision of Prof. dr. Ellen Blaak, Dr. Emanuel Canfora and Dr. Johan Jocken. His research focused on the role of gutderived metabolites (SCFA and BCFA) in in vivo metabolic health in human crosssectional studies with overweight and obese individuals as well as the role of major SCFA in human derived adipose and skeletal muscle tissues in in vitro models. During his $\mathrm{PhD}$, Manuel has presented his work as oral and poster presentations in national (Netherlands Association for the study on obesity, NASO 2018 and 2019) and international (European Congress on Obesity, ECO 2018 and 2019) conferences. In addition, he was awarded travel grants from NASO to attend ECO in 2018 and 2019. Recently, he was awarded the NASO publication prize in 2020 for the manuscript entitled "Circulating but not fecal short-chain fatty acids are related to insulin sensitivity, lipolysis and GLP-1 concentrations in humans". 


\section{List of Publications}

Manuel A. González Hernández, Emanuel E. Canfora, Kenneth Pasmans, Arne Astrup, W.H.M. Saris and Ellen E Blaak. The Relationship between Circulating Acetate and Human Insulin Resistance before and after Weight Loss in the DiOGenes Study. Nutrients. 2020; 12 (2): 339

Manuel A. González Hernández, Emanuel E. Canfora, Johan W E Jocken and Ellen E Blaak. The Short-Chain Fatty Acid Acetate in Body Weight Control and Insulin Sensitivity. Nutrients. 2019; 11 (8): 1943

Mattea Müller*, Manuel A. González Hernández*, Gijs G.H. Goossens, Dorien Reijnders, Jens J. Holst, Johan W.E. Jocken, Emanuel E. Canfora and Ellen E Blaak. Circulating but not fecal short-chain fatty acids are related to insulin sensitivity, lipolysis and GLP-1 concentrations in humans. Scientific Reports 2019; 9(1):1-9. *Shared first authors

Johan W.E. Jocken, Manuel A. González Hernández, Nicole T.H. Hoebers, Christina M. van der Beek, Yvonne P.G. Essers, Ellen E Blaak, Emanuel E. Canfora. Short-chain fatty acids differentially affect intracellular lipolysis in a human white adipocyte model. Frontiers in Endocrinology 2018; 8:372

Elisa Cirillo, Martina Kutmon, Manuel A. González Hernández, Tom Hooimeijer, Michiel E Adriaens, Lars M T Eijssen, Laurence D Parnell, Susan L Coort, Chris T Evelo. From SNPs to pathways: Biological interpretation of type 2 diabetes (T2DM) genome wide association study (GWAS) results. PLoS One 2018 13(4): e0193515

Manuel A. González Hernández, Ellen E Blaak, Nicole T. H. Hoebers, Yvonne P. G. Essers, Emanuel E. Canfora and Johan W. E. Jocken. 133. Acetate does not affect palmitate oxidation and AMPK phosphorylation in human primary skeletal muscle cells. In preparation for submission.

Manuel A. González Hernández, Emanuel E. Canfora and Ellen E Blaak. Fecal microbial metabolites of proteolytic and saccharolytic fermentation in relation to degree of insulin resistance in overweight and obese individuals. Submitted 


\section{Awards and grants}

"Publication prize 2020" for PhD students, granted by Netherlands Association for the Study on Obesity (NASO), Online meeting, The Netherlands.

TITLE: Circulating but not fecal short-chain fatty acids are related to insulin sensitivity, lipolysis and GLP-1 concentrations in humans. Scientific Reports

“NASO Travel grant 2019" for PhD students to attend European Congress of Obesity in Glasgow 2019, granted by Netherlands Association for the Study on Obesity (NASO), Online meeting, The Netherlands

"NASO Travel grant 2018" for PhD students to attend European Congress of Obesity in Vienna 2018, granted by Netherlands Association for the Study on Obesity (NASO), Online meeting, The Netherlands

Scholarship granted to Manuel Gonzalez by Consejo Nacional de Ciencia y Tecnologia (CONACYT), a public agency of Mexico's federal government to pursue master and $\mathrm{PhD}$ degrees in Biomedical Sciences. 


\section{Poster and oral presentations}

Oral presentation, Organ Cross Talk session at $\mathbf{2 6}^{\text {th }}$ European Congress on Obesity, $28^{\text {th }}$ April to May $1^{\text {st }} 2019$, Glasgow, Scotland

TITLE: Circulating but not fecal short-chain fatty acids are related to insulin sensitivity, lipolysis and GLP-1 concentrations in humans.

Oral presentation, annual meeting Netherlands Association for the Study on Obesity (NASO) 2019, Utrecht, The Netherlands

TITLE: Circulating but not fecal short-chain fatty acids are related to insulin sensitivity, lipolysis and GLP-1 concentrations in humans.

Oral poster and pitch presentation, at Annual NUTRIM School of Nutrition and Translational Research, November 2018, Maastricht, The Netherlands

TITLE: Circulating but not fecal short-chain fatty acids are related to insulin sensitivity, lipolysis and GLP-1 concentrations in humans.

Poster presentation, at $\mathbf{2 5}^{\text {th }}$ European Congress on Obesity, May 2018, Vienna, Austria

TITLE: The Relationship between Circulating Acetate and Human Insulin Resistance before and after Weight Loss in the DiOGenes Study

Oral presentation, annual meeting Netherlands Association for the Study on Obesity (NASO) 2018, Utrecht, The Netherlands.

TITLE: The Relationship between Circulating Acetate and Human Insulin Resistance before and after Weight Loss in the DiOGenes Study 